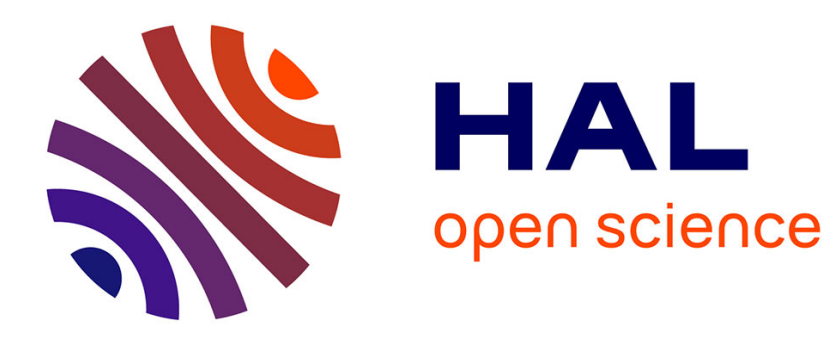

\title{
Vers la fin du temps de travail?
}

Jens Thoemmes

\section{To cite this version:}

Jens Thoemmes. Vers la fin du temps de travail?. Presses Universitaires de France, pp.225, 2000, Le

Travail Humain, 2-13-050549-X. hal-00936455v2

\section{HAL Id: hal-00936455 \\ https://hal.science/hal-00936455v2}

Submitted on 5 Aug 2014

HAL is a multi-disciplinary open access archive for the deposit and dissemination of scientific research documents, whether they are published or not. The documents may come from teaching and research institutions in France or abroad, or from public or private research centers.
L'archive ouverte pluridisciplinaire HAL, est destinée au dépôt et à la diffusion de documents scientifiques de niveau recherche, publiés ou non, émanant des établissements d'enseignement et de recherche français ou étrangers, des laboratoires publics ou privés. 
Thoemmes J., 2000, Vers la fin du temps de travail? Paris, Presses Universitaires de France (Le Travail Humain), XII-225 p.

Jens Thoemmes

VERS LA FIN DU TEMPS DE TRAVAIL ? 
I. UNE NOUVELLE CONCEPTION DU TEMPS : LE TEMPS DE TRAVAIL ET DU GAIN

$\begin{array}{ll}\text { I.1. LE TEMPS DES MARCHANDS EMERGE AU MOYEN AGE } & 17\end{array}$

$\begin{array}{ll}\text { I.1.A. Le temps des affaires } & 17\end{array}$

I.1.B. Le temps des hommes se détache du temps naturel et du temps de l'église $\quad 18$

I.2. LE TEMPS DES MARCHANDISES : UNE AUTRE MANIERE DE VOIR LE TEMPS AU XIXE SIECLE 19

I.2.A. Les trois composantes du temps de travail : durées, horaires et rythmes 20

I.2.B. Caractérisation du temps comme marchandise : subordination et rationalisation 23

I.2.C. Temps monochrone et ethos de la besogne 25

II. LA CONSTRUCTION DE L'ORDRE TEMPOREL PAR LES REGLES : LA NORME TEMPORELLE 27

II.1. LE CORPS FAIT IRRUPTION DANS L'ORDRE JURIDIQUE 27

II.1.A. Nécessité d'une loi pour protéger la santé selon Villermé. 28

II.1.B. Députés et sénateurs s'entendent en 1839 pour éviter une loi qui limiterait la durée du travail 30

II.2. LA LENTE MONTEE DE LA REGLEMENTATION SUR LE TEMPS DE TRAVAIL

II.2.A. L'allongement de la durée du travail et la première loi de 1841 : l'intervention de l'État 31

II.2.B. Un demi-siècle pour aboutir aux 10 heures par jour (1841-1904) : des inspecteurs pour faire respecter la loi

II.2.C. La norme temporelle pour tous (1900-1980) : 40 heures par semaine, $8 \mathrm{~h}$ par jour, repos de fin de semaine et les congés payés

II.2.D. La norme temporelle : 150 ans d'histoire 34

III. LES PRATIQUES $\quad 38$

III.1. LA DUREE DU TRAVAIL DE QUOI PARLE-T-ON ? 39

III.2. LA DUREE HEBDOMADAIRE DU TRAVAIL ENTRE 1891 ET 1995 EN FRANCE : 4 PHASES 41

III.2.A. Une période de forte réduction de la durée du travail de 65,2 heures à 40 heures (25,2 heures) : 1891-1936

III.2.B. Une période d'augmentation de la durée du travail de 40 heures à 45 heures (+ 5 heures) : 1937-1966

III.2.C. Une période de réduction de la durée hebdomadaire du travail de 45 heures à 39 heures : 1967-1983

III.2.D. La décennie après les lois Auroux : la stabilité de la durée hebdomadaire à 39 heures 
III.3. L'EUROPE ET LA FRANCE : UNE EVOLUTION COMPARABLE DES DUREES HEBDOMADAIRES DU TRAVAIL ?

III.3.A. La codification du temps de travail à la lumière des durées pratiquées $\quad 46$

$\begin{array}{lll}\text { III.3.B. France et Union Européenne } & 47\end{array}$

IV. CONCLUSION

IV.1. UNE CONCEPTION DU TEMPS CENTREE SUR LE TEMPS INDUSTRIEL

IV.2. L'IMPORTANCE DE LA REGLEMENTATION DU TEMPS DE TRAVAIL

IV.3. LA DUREE COMME FIGURE EMBLEMATIQUE DU TEMPS DE TRAVAIL 52

IV.4. DUREES PRATIQUEES : BAISSE SUR UN SIECLE, MAIS QUATRE PHASES CONTRADICTOIRES 53

CHAPITRE II L'ECLATEMENT DU TEMPS DE TRAVAIL

I. L'OUVERTE DE L'ESPACE DE NEGOCIATION : LE CONTEXTE JURIDIQUE $\mathbf{5 8}$

I.1. UN CHANGEMENT DE LA CONCEPTION DU TEMPS DE TRAVAIL 58

I.1.A. De la conception hygiéniste à la conception gestionnaire 59

I.1.B. Du corps à l'emploi 59

I.2. UN CHANGEMENT DU MODE DE REGULATION : DU DECRET A LA NEGOCIATION 60

$\begin{array}{ll}\text { I.2.A. Le tournant de } 1981 & 60\end{array}$

I.2.B. L'espace de négociation : durée, flexibilité, emploi 61

I.3. UN CHANGEMENT DE LA NATURE DES REGLES TEMPORELLES

II. L'ECLATEMENT DU TEMPS DE TRAVAIL : LA NEGOCIATION ENTRE 1984 ET 1994

II.1. UNE FORTE ADHESION AUX ACCORDS SUR LE TEMPS DE TRAVAIL 66

II.2. LE TEMPS, MONNAIE D'ECHANGE OU OBJET DE MARCHANDAGE? 66

II.3. LES 6 FIGURES DU TEMPS DE TRAVAIL : TEMPS DES MARCHES, TEMPS DES MACHINES, TEMPS ECHANGE, TEMPS INDIVIDUALISE, TEMPS DES CONGES ET TEMPS CONFIRMES 67

$\begin{array}{ll}\text { II.4. RESUME } & 71\end{array}$

III. VERS L'INTENSIFICATION DISCONTINUE DU TRAVAIL?

III.1. LE COMPTE EPARGNE TEMPS (CET) : UNE LOI POUR MIEUX GERER LES ACTIVITES 73

III.2. LE CET COMME « SOUPAPE » ET COMME CADRE TEMPOREL PLURIANNUEL 73

III.3. L'EMERGENCE D'UN TEMPS VIRTUEL

III.4. LE CET ET LA FLEXIBILITE TEMPORELLE

IV. DISCUSSION ET CONCLUSION

IV.1. $\quad$ LA PERTE DE CENTRALITE DE LA DUREE DU TRAVAIL

IV.2. LES FIGURES RECENTES DU TEMPS DE TRAVAIL SONT ORIENTEES VERS LA LOGIQUE DE LA $\begin{array}{ll}\text { PRODUCTION } & 77\end{array}$

IV.3. DE L'ECLATEMENT A L’INTENSIFICATION DISCONTINUE DU TRAVAIL ? 


\section{LE TRAVAIL DU SAMEDI ET LE CHANTAGE A L'EMPLOI : LE CAS DE L'ENTREPRISE CLARO}

I.1. BLOCAGE DANS LA NEGOCIATION AU REGARD DU TRAVAIL DU SAMEDI

I.2. LA NEGOCIATION DE 1995 ET 1996 : LES SYNDICATS CHANGENT DE POSITION PAR RAPPORT AU TRAVAIL DU SAMEDI

I.2.A. Le blocage de la négociation par le recours au référendum 88

I.2.B. L'intervention du plan social pour débloquer la négociation 89

I.3. TRAVAIL DU SAMEDI SOIR ET L'EMPLOI 90

II. LE TEMPS DE TRAVAIL DES CADRES ET LES CADRES DU TEMPS DE TRAVAIL : LE CAS DE SINUS 92

II.1. A LA RECHERCHE DU TEMPS DE TRAVAIL DES CADRES 94

II.1.A. Forfaitaires ou mensualisés : une distinction devenue obsolète 94

II.1.B. Le ras le bol des cadres face à la pression temporelle 96

II.1.C. Réduction contre productivité : vers la semaine de quatre jours ? 96

II.2. LE TEMPS PARTIEL : SOLUTION LIMITEE POUR CADRER LE TEMPS DES CADRES 97

II.2.A. Des normes temporelles plus conformes aux pratiques des cadres et au contexte légal. 98

II.2.B. Une carrière redéfinie : l'histoire d'Adrien $\quad 99$

$\begin{array}{ll}\text { II.2.C. Du travail au temps } & 101\end{array}$

III. LE TRAVAIL DE NUIT : FAUT-IL LE RESERVER AUX HOMMES? LE CAS DE FANA 101

III.1. L'ARRANGEMENT TEMPOREL EST UNE CONSTRUCTION SOCIALE : LE TRAVAIL DE NUIT EST NEGOCIE. 104

III.1.A. Faire accepter le travail de nuit par le volontariat 104

III.1.B. Le référendum pour infléchir la négociation 105

III.2. DES REPRESENTATIONS SEXUEES DU TEMPS JALONNENT LA NEGOCIATION DE L'ARRANGEMENT TEMPOREL

III.2.A. La dévalorisation relative des qualifications de la main-d'œuvre féminine : progrès techniques et interdiction du travail de nuit

III.2.B. La concordance des temps sociaux au cœur des représentations sexuées du temps 107

III.2.C. Le travail de nuit des femmes : un « seuil de tolérance » pour maintenir les femmes à l'écart? 109

IV. L'ANNUALISATION ET L'INCOMPATIBILITE DES HORIZONS TEMPORELS REELS : LE CAS DE ROTOR 112

IV.1. TOUTES LES CAISSIERES SONT A TEMPS PARTIEL

IV.2. L'ANNUALISATION DU TEMPS PARTIEL : UNE SORTIE DU DILEMME DE LA GESTION DES DUREES DU TRAVAIL?

IV.2.A. Négocier le temps partiel annualisé avec un syndicalisme réduit 
IV.2.B. Codification annuelle du temps à risques : une coalition tacite pour ne pas appliquer la règle. 116

\section{DU TEMPS DE TRAVAIL AU TEMPS DE L'EMPLOI}

V.1. L'EMPLOI « CONTRAINTE » DE LA NEGOCIATION DU TEMPS DE TRAVAIL

V.2. L'EMPLOI « CATALYSEUR » DE LA NEGOCIATION SUR LE TEMPS DE TRAVAIL 120

V.3. L'EMPLOI COMME « ECRAN » : UNE NEGOCIATION DU TEMPS DE TRAVAIL BLOQUEE 122

VI. CONCLUSION 125

VI.1. LA CONSTRUCTION DES ARRANGEMENTS TEMPORELS 125

VI.2. LES ARRANGEMENTS TEMPORELS COMME OUTIL DE LA GESTION DE L'EMPLOI 126

VI.3. LE TEMPS DE TRAVAIL INCORPORE UNE DOUBLE RATIONALITE

\section{SYNDICALISME ET NEGOCIATION COLLECTIVE DU TEMPS DE TRAVAIL}

I.1. NEgOCiation COLLECTIVE ET REgULATIONS SOCIALES 131

I.2. LE TEMPS DE TRAVAIL ET L'ACTION SYNDICALE 132

I.2.A. Le temps de travail comme pivot de l'action syndicale 134

I.2.B. Pourquoi s'intéresser à la négociation du temps de travail ? 135

I.2.C. Le référentiel de négociation : comment interpréter la nature, la portée et la solidité des accords? 136

II. DYNAMIQUE ET CONTRADICTION DE L'ACTION SYNDICALE $\quad 137$

$\begin{array}{lll}\text { II.1. } & \text { LA DISTINCTION DES PRATIQUES SYNDICALES } & 138\end{array}$

II.1.A. Du comportement de signature à l'engagement dans la négociation 139

II.1.B. Un engagement dans la négociation différent selon les syndicats et les thèmes 140

II.2. DES REFERENTIELS NEGOCIATOIRES SINGULIERS 142

II.2.A. La CFDT : partage du travail et flexibilité contrôlée 143

II.2.B. La CFTC : solidarité et protection de la famille 146

II.2.C. La CGC : la semaine de 4 jours et l'acceptation de la flexibilité 148

II.2.D. La CGT : plein-emploi et stabilité des repères temporels 152

II.2.E. FO : Contractualisation et résistance à la guerre économique $\quad 154$

$\begin{array}{ll}\text { II.2.F. Conclusion } & 157\end{array}$

II.3. LES SYNDICATS UNIS CONTRE UN ORDRE TEMPOREL EXCLUSIVEMENT ORIENTE VERS LA PRODUCTION 158

II.3.A. Avoir du temps pour soi et du travail pour tous 158

II.3.B. Stabilité des repères temporels et retour sur la famille 160

II.3.C. Maîtriser l'usage du cadre temporel de travail et préserver son emploi 161 
I. PARTAGE DU TRAVAIL : DIVISER OU REPARTIR?

II. LA SEMAINE DE 4 JOURS CHEZ VW : UN ACCORD EN DEUX TEMPS

II.1. LES RELATIONS PROFESSIONNELLES EN ALLEMAGNE

II.2. CHRONIQUE D'UN ACCORD ECLAIR ET FONDATEUR : DU 1ER OCTOBRE AU 15 DECEMBRE 1993169

II.2.A. La « semaine de 4 jours » : les difficultés de la mise en oeuvre

II.2.B. La « semaine de 4 jours » au département tôlerie de l'usine de Hanovre : la pénurie de main-d'oeuvre

II.2.C. L'accord et ses limites : ouverture d'un débat social sur l'irruption d'un nouveau paradigme de la négociation

II.3. CONFIRMATION ET SEUIL DE DECROCHAGE DU PARTAGE DU TRAVAIL

II.3.A. Chronique de la négociation suivante : changement des termes de l'échange et conflit 177

II.3.B. Du conflit au compromis : les limites de la rationalisation sont dans la rue

II.3.C. L'emploi reste au cœur de l'accord : interdiction de licenciements indéterminée et obligation d'embauche des apprentis

II.3.D. De la semaine de 4 jours à 5 jours par semaine 180

II.3.E. Vers le travail du samedi ou vers un cadre pluriannuel ? 181

II.3.F. Flexibilité et déstabilisation des collectifs de travail 182

II.3.G. Travailler plus pour maintenir l'emploi 183

III. LA DIFFUSION DU PARTAGE « VW » EN ALLEMAGNE : 1994-1996 186

III.1. LE CAS DE L'ENTREPRISE MECANIQUE BERGO 186

III.1.A. Contexte et chronique de la négociation 186

III.1.B. Mythe et réalité de l'accord fondateur chez VW pour les acteurs de BERGO 187

III.1.C. La solution retenue : partage du travail, perte salariale et flexibilité 187

III.1.D. Les 32 heures, un cadre flexible pour les durées, mais rigide pour les salaires 188

III.2. L'IMPORTANCE DES REGULATIONS INTERMEDIAIRES POUR LA DIFFUSION DU PARTAGE DU TRAVAIL 189

III.2.A. Bergo : un partage du travail dans le dos d'IG Metall

III.2.B. La montée des régulations intermédiaires du temps de travail en Allemagne depuis VW 191

III.3. LE NIVEAU GLOBAL S'APPROPRIE L'ARRANGEMENT VW POUR LE GENERALISER

IV. PARTAGE ET FIN D'Un TEMPS DE TRAVAIL EN Allemagne?

IV.1. VW INTRODUIT UN PARADIGME CONCURRENTIEL DE LA NEGOCIATION DU TEMPS DE TRAVAIL QUI SE DIFFUSE EN ALLEMAGNE 
IV.2. L'ACCEPTATION DE LA PERTE SALARIALE 194

IV.3. LA FLEXIBILITE AUGMENTE 195

IV.4. LE PARADIGME DU PARTAGE MET AU CENTRE L'EMPLOI 195

IV.5. LA PERCEE DES REGULATIONS INTERMEDIAIRES DANS LES RELATIONS PROFESSIONNELLES 196

IV.6. LES TEMPORALITES SOCIALES SONT MISES EN CAUSES, MAIS LEUR DESTRUCTION N’EST PAS ACQUISE 197

IV.7. LE PARTAGE DU TRAVAIL : UNE SOLUTION COMMUNAUTAIRE POUR MAINTENIR L'EMPLOI? 198

CHAPITRE VI LA SUBSISTANCE ET LA FIN D'UNE NORME TEMPORELLE

I. EFFONDREMENT D'UNE CONCEPTION DU TEMPS DE TRAVAIL 201

I.1. LA PERTE DE LA CENTRALITE DE LA DUREE HEBDOMADAIRE DU TRAVAIL ET L'AVENEMENT DU TEMPS

$\begin{array}{ll}\text { DES MARCHES } & 202\end{array}$

I.2. DES REPRESENTATIONS DIVERSES SUR FOND COMMUN DE RESISTANCES 204

II. LA FRAGMENTATION DE L'EMPLOI : LE TEMPS REDUIT OU RALLONGE 205

II.1. LA FRAGMENTATION DIRECTE : LE TEMPS PARTIEL 205

II.2. LA FRAGMENTATION INDIRECTE : LA BAISSE DES SALAIRES 206

III. EMPLOIS MULTIPLES : LE TEMPS ADDITIONNE 209

III.1. L'EMPLOI MULTIPLE EN DEBUT DE SIECLE 210

III.2. LES « CONTINGENT WORKERS » ET LE CUMUL DES EMPLOIS 211

IV. TEMPS DE LA SUBSISTANCE OU TEMPS PROTEGE?

\begin{tabular}{lr} 
BIBLIOGRAPHIE & 218 \\
\hline
\end{tabular} 


\section{INTRODUCTION GENERALE}

Cet ouvrage voudrait s'inscrire dans un double débat. Premièrement dans le débat touchant les pratiques de définition et de mise en oeuvre du temps de travail : sa réduction ou réorganisation, ses effets sur les temps sociaux qui incluent le temps hors travail et ses effets sur la santé ou sur l'emploi. Ce dernier enjeu reste au centre des préoccupations dans le débat sur le temps de travail. Le chômage issu des licenciements collectifs continue à constituer un problème important, malgré le nouveau cadre législatif que fournit la loi Aubry (1998) en vue de réduire la durée légale à 35 heures. Les heures supplémentaires, le travail de nuit et de week-end, le temps partiel, la modulation-annualisation et le compte épargne temps sont des codifications qui recouvrent des enjeux réels de compatibilité des temps sociaux, parmi lesquels le temps de travail. Ce débat ne peut pas donc se réduire à la seule question de l'emploi. Nous voudrions aborder la négociation du temps de travail en vue de comprendre comment se construit le cadre temporel des activités professionnelles qui mêle tous ces éléments des conditions de travail : emploi, flexibilité, perte salariale, réduction de la durée du travail. Nous nous proposons de voir ce que recouvrent ces termes dans le cas de la négociation.

Le second débat dans lequel s'inscrit cet ouvrage concerne les conceptions du temps de travail : il nous semble qu'on arrive à la fin d'une certaine conception du temps de travail ; d'où le titre « Vers la fin d'un temps de travail ?».

L'étude du temps de travail place d'emblée le problème du rapport au travail sur le terrain de son cadrage temporel. A partir de cet angle d'attaque le travail se retrouve inséré dans un complexe d'activités de loisirs, domestiques et d'autres. Nous montrerons que la négociation collective influence l'équilibre «temporel» de ces activités. Nos analyses portent alors sur l'arrangement temporel et ses rapports aux temps réglementés, consacrés ou représentés. Dans tous les cas, nous observons que les querelles sur la gestion de l'emploi du temps constituent un élément central de l'organisation des activités professionnelles. De plus, ces conflits et compromis ont souvent lieu derrière des portes fermées : il s'agit de tenter avec les acteurs d'ouvrir ces portes et de mettre en discussion les manières de pratiquer le temps de travail. Le travail, monde de l'implicite, ne dévoile pas aisément les manières de se disputer le temps et de trouver des compromis sur le déroulement des activités. Une visite d'entreprise, une observation ou d'une série d'entretiens ne permet que dévoiler un peu plus la face invisible de l'iceberg. L'occupation du temps, pour reprendre Naville (1972), est toujours étroitement liée au travail et au lien de subordination à l'employeur, institué par le contrat de travail. Le contrat comme 
principe de cadrage temporel confère à l'employeur le pouvoir de disposer du temps des employés. Pourtant le caractère de plus en plus négocié du cadre temporel pourrait laisser croire que les solutions issues de ces compromis négociés améliorent la qualité de vie au travail. Une telle affirmation doit être nuancée. Le fait que les possibilités d'intervention sur le temps de travail se sont fortement accrues pour les organisations syndicales depuis le début des 1980, n’a pas écarté les situations où l'individu reste exposé à des conditions de travail difficiles et à des problèmes de santé liés à un travail répétitif. Ces observations confirment ce que nous voudrions expliciter pour le caractère socialement structurant du temps pour le travail : le temps de travail continue à être une contrainte forte de la vie sociale.

La multiplicité de questionnements, la diversité de situations analysées, l'hétérogénéité de matériaux recueillis, la variété des formes d'analyse n'est pas le résultat du hasard, mais le résultat de choix que nous avons faits durant ces recherches. Ces choix ont été établis au regard de trois types de questions qui finalement ont structuré notre pratique de recherche. La première question concerne le contexte de la production des formes temporelles et de leur nature; la seconde concerne les chemins de la production des formes temporelles et les raisons pour lesquelles de tels chemins sont empruntés. Enfin, la troisième concerne les rapports que ces formes temporelles de travail entretiennent avec la société toute entière et les diverses temporalités qui la traversent.

La première question relative à la nature et au contexte de la production des formes temporelles a constitué notre première étape de recherche : les pratiques de la négociation (les accords d'entreprise sur le temps de travail) ont été mises en relation avec le contexte de la négociation, à savoir son cadre juridique. Nous avons tenté de mettre en relation l'évolution conjointe des pratiques et des règles juridiques, de façon à comprendre non seulement ce que fait la loi aux individus, mais ce qu'ils en font: l'évolution des règlements et l'analyse de douze ans de négociations collectives sur le temps de travail dans une région de France en ont constitué le matériau.

La deuxième question est relative aux chemins empruntés, voire ouverts pour arriver à ces compromis temporels. En effet, les « accords » analysés constituent le résultat « froid» de négociations dont il fallait comprendre la dynamique et les modalités. En faisant son entrée sur la scène de la négociation, le temps de travail était l'occasion pour les négociateurs d'ouvrir un espace de négociation et pour produire des formes temporelles originales : l'analyse monographique d'une dizaine d'entreprises en a constitué le matériau. Mais l'analyse des chemins de la négociation nous a conduit à développer la notion de « référentiel négociatoire» pour caractériser la dimension cognitive de la négociation : 
c'est alors que nous avons pris le parti de développer des entretiens approfondis, avec des responsables syndicaux engagés dans des négociations concrètes ou bien ayant une position de responsable dans les organisations syndicales; c'est ce que nous avons fait, tant en France qu'en Allemagne.

La troisième question est relative à l'articulation entre temps de travail et société. Si la négociation collective a constitué un angle privilégié pour analyser la nature et les modalités de production des formes temporelles, les enjeux de cette production normative portent la marque de leur inscription dans la société toute entière. D'une part, parce que le temps de travail est l'une des composantes des temporalités sociales qui en comporte bien d'autre, d'autre part parce que les configurations visant à articuler ces temporalités ne sont pas identiques d'une société à l'autre.

Il ne s'agissait pas pour nous de procéder à des comparaisons internationales, mais plutôt d'éviter deux impasses : le premier était de croire que les configurations temporelles ne sont ordonnées qu'en fonction du travail et en tournant le dos aux autres temporalités; le second était de généraliser, à partir de la France, des résultats qui portent la marque de la société dans laquelle ils ont été produits.

C'est pourquoi, nous avons d'une part, cherché à comprendre comment les acteurs essayent d'assurer une «concordance des temps» (Supiot, 1995) voire de limiter la discordance entre des temporalités multiples; d'autre part nous avons cherché à saisir dans d'autres sociétés sur quelle manière de voir le temps de travail, des compromis temporels pouvaient être imaginés.

Notre recherche nous amène à mettre en discussion l'idée qu'il y a un profond remaniement dans la constitution et la nature de l'ordre temporel qui encadre les activités de travail. De quoi s'agit-il ? Ni d'une réponse affirmative, ni d'un résultat figé, mais d'une tendance que nous souhaitons soumettre à la discussion.

Dans cet ouvrage, nous décrivons la dynamique de construction des normes temporelles touchant le travail avec deux étapes distinctes. La première concerne la constitution de ces normes temporelles combinant des pratiques, des représentations et des règlements. La seconde concerne l'éclatement et la recomposition des normes temporelles.

La fin d'un temps de travail concerne la fin d'une certaine conception du temps de travail : un temps "protégé », c'est-à-dire limité et légitime faisant l'objet d'un consensus qui s'était lentement élaboré au fil des années. Il s'agit donc en reprenant le terme de Max Weber d'un véritable « désenchantement» concernant le rapport entre temps, activités et sociétés. Le consensus devenu «norme» vole en éclat : d'où la nécessité de s'interroger sur la recomposition des temps de travail et des temps sociaux. Quel est ce nouveau temps 
de travail? Quelle est sa cohérence et en quoi peut-il être considéré comme "norme »? Quelle place réserve-t-il à l'épanouissement des individus au travail et hors travail? Comment répartit-il ceux qui possèdent un emploi et ceux qui n'en ont pas?

Il nous semble et c'est notre thèse que ce processus aboutit à une polarisation : pour les uns, nous assistons à l'émergence d'un nouveau temps protégé, caractérisé certes par la flexibilité et par le maintien de l'emploi. Pour les autres, nous constatons l'apparition d'un temps non-protégé, de la pluriactivité professionnelle, de la recherche d'heures complémentaires, bref d'un temps de la subsistance.

Nous avons tenté d'ordonner les résultats de notre recherche en présentant les principaux éléments pour comprendre « où va le travail humain ?», pour reprendre la formule de Friedmann (1963) à propos du travail. Nous avons essayé d'une part, de procéder à un découpage afin de garder la cohérence dans la progression des raisonnements et d'autre part, d'appuyer l'argumentation par les données recueillies en évitant de présenter la totalité de ces données. Ce double fil conducteur de l'exposé nous a conduit à présenter les différentes faces du temps de travail, tel que nous l'avons abordé.

Disons le tout de suite la fin d'un temps de travail ne signifie pas la fin du travail et des activités professionnelles. Notre approche vise à montrer que la fin d'un temps de travail peut tout à fait cohabiter avec la réduction de la durée du travail sans pour autant relâcher sa prise sur les autres temps sociaux. La fin d'un temps de travail telle que nous la voyons à l'œuvre est un processus lent, certes réversible, et qui vise à recomposer les temps sociaux. Il est loin d'être sûr que cette recomposition se traduit par un plus d'autonomie pour les individus. Le nouvel temps qui se substitue lentement au temps de travail pourrait se caractériser précisément par une domination plus prononcée de ce temps sur les autres temps sociaux.

Parler d'une fin, présuppose qu'il y a un début ou un commencement. Bien entendu, les activités professionnelles se sont toujours déroulées dans un cadre temporel : il serait donc illusoire d'en chercher le commencement. Pourtant, nous avons cherché à comprendre comment le temps de travail s'institutionnalise pour devenir un temps dominant: s'il y a bien dés le Moyen Age, une conception du temps de travail qui se met en place, il faudra attendre en France le XIXe siècle pour voir l'émergence du temps structuré par des règlements. Ce «temps légal » mettra 150 ans, de 1830 à 1980, pour se structurer et avoir sa figure dominante caractérisée par (a) les normes temporelles, (b) une consécration de l'État comme puissance normative, (c) des règles communes, enfin (d) un dispositif de 
sanction. Ce temps de travail devient le cadre des activités de travail en même temps que le principe de leur organisation.

Pourtant, dans une période récente cette structure temporelle va se fissurer pour donner lieu à une pluralité de configurations temporelles qui accompagnent les activités de travail. Cela signifie-t-il que le temps de travail n'est plus la structure temporelle dominante ? Ne assiste-t-on pas à l'émergence d'un « temps éclaté »? C'est ce que nous voudrions montrer afin de comprendre en quoi l'entrée du temps de travail dans le champ de la négociation collective conduit à une remise en cause de la structure temporelle qui s'était établie antérieurement. Quelle est la signification de ces configurations temporelles ? Elles sont orientées vers la flexibilité, c'est-à-dire vers l'ordre de la production : mais sont-elles seulement tournées vers la production? Ou bien, incorporent-elles aussi et de quelle manière l'ordre de la vie quotidienne des salariés ? Y a-t-il un conflit de rationalité ? L'analyse des dispositifs réglementaires et des pratiques de négociation sur le temps de travail pendant 15 ans sur une région, nous aidera à répondre à ces questions.

L'analyse monographique, sur plusieurs sites, nous a permis de suivre les chemins de la négociation collective sur le temps de travail, afin de comprendre les raisons pour lesquelles cette variété de compromis, a pu se mettre en place, en combinant deux rationalités. Si la question de l'emploi est fondamentale pour comprendre ce qui est au cœur de l'échange entre employeurs et salariés, il faut aussi comprendre l'importance des contextes locaux et des situations singulières pour en établir la pertinence. Ces études de cas montrent au moins, que le champ de la négociation du temps de travail s'est ouvert pour devenir un champ de discussions intenses et de compromis variés, que nous appelons « le temps arrangé », dans lequel l'emploi joue un rôle de menace pour déclencher et faire aboutir la négociation. La notion « d'arrangement temporel » nous invite à expliciter son fondement: s'agit-il seulement d'un compromis local et d'une production normative interne à l'entreprise ? Ou bien, peut-on considérer que ces arrangements sont porteurs de solutions qui pourraient se développer ailleurs?

La notion de « référentiels négociatoires » nous servira à comprendre, la nature et le rôle de ce que nous appelons «le temps représenté ». Quels sont ces référentiels et quels en sont les composantes? Les représentations du temps de travail véhiculées par les organisations syndicales ne sont pas homogènes mais singulières et renvoient au système de valeurs propre à chaque syndicat. Quel est le rôle des référentiels dans la construction d'une action collective ? Joue-t-il un rôle de ciment pour souder les protagonistes ou bien cette singularité du référentiel divise-t-elle les syndicats ? Sans doute les deux; mais dans 
tous les cas, il oriente les pratiques négociatoires et définit les alliances et les exclusions entre les acteurs.

Que le temps de travail soit négocié en incluant dans le champ les questions d'emploi, nous invite à expliciter les conséquences de cette mise en relation sur la notion de temps de travail. Le temps de travail n'est-il pas alors redéfini ? Ne constitue-t-il pas un moyen pour obtenir autre chose, à savoir le maintien de l'emploi ? C'est ce que nous tentons de montrer en explicitant la notion de «partage flexibilisé du travail » et de sa diffusion dans un autre contexte, tel qu'elle résulte de l'analyse des accords sur le temps de travail de la métallurgie en Allemagne. Nous tentons de reconstituer à l'aide d'exemples, la dynamique de diffusion d'un arrangement temporel à propos de l'emploi, afin de comprendre les déterminants, les aboutissements d'un accord tant du point de vue interne et externe à l'organisation productive. Dès lors, on peut se demander, si le temps de travail reste le cadre et le principe d'organisation des activités, comme un outil de gestion au service de l'emploi.

Le temps de travail a été abordé jusqu'ici comme un objet négocié, ce qui nous a permis d'expliciter les termes de l'échange entre employeurs et salariés : le triangle emploi flexibilité - réduction de la durée, était au cœur de cet échange. Mais le temps de travail n'est-il que cet objet que les négociateurs se disputent? Qu'en est-il des situations ou le travail s'écarte des normes d'emploi habituelles? Il nous semble que la notion de temps de travail doit être situé dans un espace plus vaste. Les hiérarchies se définissent en fonction $\mathrm{du}$ statut d'emploi. Comment des personnes qui travaillent, habitent-elles ces cadres temporels, dès lors que leur statut d'emploi est précaire ? L'exploration du temps de travail aux Etats Unis, comme en Russie nous a aussi permis d'analyser d'autres formes de compromis : il ne s'agit pas d'échanger une réduction de la durée contre de la flexibilité ou contre le maintien de l'emploi, mais peut être tout simplement d'obtenir des ressources financières pour survivre. C'est ce que nous avons appelé « le temps de la subsistance », pour désigner un espace d'action qu'il faut gérer moins pour réduire la durée du travail, que pour obtenir du travail, donc des heures de travail rémunérées afin de subsister. Nous aurions pu trouver des situations similaires en Europe à interpréter comme un temps de la subsistance.

Bien entendu la catégorisation du temps de travail en six temps distincts (temps des marchés, temps individualisés, temps des machines, temps échangés, temps des congés et temps confirmés) à laquelle nous aboutissons avec l'étude des accords conclus en France au niveau régional, ne doit pas laisser penser qu'il s'agit de situations obligatoirement 
indépendantes les unes des autres. De la même manière, cette simplification ne doit pas laisser sous entendre qu'il n'y a qu'une manière d'aborder le temps de travail.

Si nous avons privilégié la négociation collective comme domaine de recherche, c'est pour expliciter les processus sociaux qui sont à l'œuvre. Ce faisant, nous avons tenté d'expliciter la place de la régulation dans la négociation, la nature des règles temporelles élaborées, les termes de l'échange et les compromis. 


\section{CHAPITRE I \\ L'INVENTION DU TEMPS DE TRAVAIL}




\section{INTRODUCTION}

Vouloir s'interroger sur la « fin d'un temps de travail», nécessite d'analyser d'abord les conditions de son émergence et de sa montée en puissance, qui va faire du temps de travail, le temps dominant et finalement la base d'un ordre temporel particulier, parce qu'il va mettre au centre les activités professionnelles. S'interroger sur l'ordre temporel dominant, c'est avant tout comprendre quelles sont les temporalités qui se combinent, afin de dégager parmi elles, celles qui englobent les autres. Cette interrogation a pour point de départ, l'existence d'un «temps dominant». Cette analyse s'inscrit dans la perspective élaborée par Grossin (1996), dont nous retiendrons l'idée d'un temps dominant qui accompagne chaque société dans la production de ses activités : le temps est à la fois ce qui cadre les activités et simultanément la représentation collective de ce temps à laquelle les individus se rangent. Ce cadre désigne l'activité principale qui s'inscrit dans un temps principal, majeur et dominant.

\section{UNE NOUVELLE CONCEPTION DU TEMPS : LE TEMPS DE TRAVAIL ET DU GAIN}

\section{I.1. Le temps des marchands émerge au Moyen Age}

La première caractérisation du temps de travail est, sans aucun doute, celle développée par Le Goff $(1977)^{1}$ à propos du temps de travail au Moyen Age et que nous résumons brièvement. Le temps de travail, c'est celui des marchands qui va s'imposer comme le cadre pertinent pour nommer et mesurer les activités professionnelles. Si tel est le cas, comment un tel cadre peut-il s'articuler avec le temps naturel ou avec le temps de l'église ? Une nouvelle manière de voir le temps mesuré et discontinu apparaît : ne va-t-elle pas écarter la conception cyclique et capricieuse du temps naturel, ainsi que la conception d'un temps religieux continu et à sens unique?

\section{I.1.A. Le temps des affaires}

De plus en plus, au Moyen Age, le temps devient dans le commerce une préoccupation. D’abord (a) pour calculer la durée des voyages, ensuite (b) pour mesurer les activités de travail et enfin (c) pour établir le prix des choses : «mais quand s'organise un réseau commercial, le temps devient objet de mesure. La durée d'un voyage par mer ou par terre d'une place à une autre, le problème des prix qui [...] haussent ou baissent, augmentent ou

1 On se reportera en particulier au chapitre intitulé : «Au Moyen Age : Temps de l'église et temps marchand, pp. 46-65,

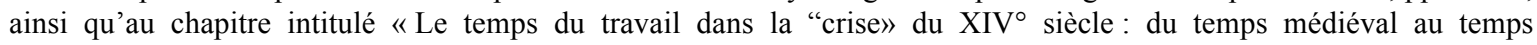
moderne », pp. 66-79. 
diminuent les bénéfices, la durée du travail artisanal et ouvrier, pour ce marchand qui est aussi presque toujours un donneur d'ouvrage - tout cela s'impose davantage à son attention, devient objet de réglementation de plus en plus précise » (Le Goff, 1977, p. 55).

Les marchands découvrent l'importance du temps qui sert à mesurer ses trajets, mais aussi à mesurer le temps de l'activité professionnelle des autres : en particulier de ceux qui vont produire les biens qu'il va vendre. Le temps des affaires devient une affaire de temps, c'est-à-dire repose sur un cadrage temporel et un instrument de mesure. Le temps et le travail sont donc bien imbriqués, le temps servant à régler le travail : plus généralement encore, le temps des marchands est un instrument de cadrage de l'activité des autres, c'està-dire de tous ceux qui servent les marchands. C'est du moins ce que nous indique Le Goff (1977, p. 56) lorsqu'il écrit : «Et pour les servir [les marchands], apparaît la nécessité d'une mesure rigoureuse du temps, car dans la draperie il convient que la plupart des ouvriers journaliers - le prolétariat du textile - aillent et viennent à leur travail à des heures fixes ». Si on suit Le Goff, tous les éléments du cadrage des activités professionnelles par le temps sont présents dès le Moyen Age. Mais quelle est la spécificité du temps de travail au regard des autres façons de structurer les activités humaines par le temps naturel, ou par le temps religieux?

\section{I.1.B. Le temps des hommes se détache du temps naturel et du temps de l'église}

Ce temps de travail qui structure explicitement les activités professionnelles repose sur une autre conception du temps. Ce temps est mesurable et surtout discontinu, coupé d'arrêts : de plus, il se détache du temps de l'église et du temps naturel.

- Tout d'abord, pour Le Goff (1977, p. 56) le temps « qui commence à se rationaliser, se laïcise du même coup » et se détache en quelque sorte du temps de l'église qui rythme les activités humaines par les offices religieux et par les cloches qui les annoncent: «a ce temps de l'église », écrit-il, «marchands et artisans substituent le temps plus exactement mesuré, utilisable pour les besognes profanes et laïques, le temps des horloges. » Il y aurait beaucoup à dire sur le rapport entre l'horloge et le clocher : tantôt, il est conflictuel, tantôt complémentaire. La relation entre l'horloge et le clocher, c'est aussi la relation entre le temps de la vie de travail et le temps de la vie religieuse. Au marchand d'arbitrer entre les gains et le salut, mais surtout entre les règles du travail et les règles de l'église.

- Ensuite, après s'être « laïcisé » le temps de travail s'affranchit des durées naturelles, de l'alternance du jour et de la nuit: il se détache du temps naturel et se présente sous 
plusieurs composants qui vont constituer les premiers éléments de la structure temporelle et en quelque sorte les prémisses du temps naturel : (a) Le travail de nuit fait l'objet déjà de mesures soit pour l'autoriser soit pour le réprimer. Au XIVe siècle et « dans ce contexte naturel et rural, le travail de nuit est une sorte d'hérésie urbaine, en général sanctionnée par interdiction et amendes » (Le Goff, 1977, p. 68). Mais par l'ordonnance du 19 janvier 1322 le prévôt de Paris autorise le travail de nuit. (b) La durée journalière qui fait l'objet de réclamations contradictoires : d'un côté en 1315 à Arras «maîtres drapiers et valets des foulons réclament des journées plus longues et des salaires plus élevés ». De l'autre, les vignerons journaliers réclament une réduction de la durée du travail qu'ils obtiennent en 1393 à l'issue d'un procès devant le Parlement de Paris. Enfin (c) la demi-journée s'affirme au XIVe siècle au travers de la pause de midi, dont on peut remarquer qu'elle peut être signalée par les cloches de l'église, donc s'articuler au temps clérical.

Ce temps perd la linéarité du temps religieux qui conduit le chrétien à Dieu; il perd aussi l'imprévisibilité de temps naturel et du temps météorologique. La conception qui émerge est celle d'un temps mesuré et discontinu qui encadre les activités professionnelles, les mesure et les organise. La pression du temps de travail n'est-elle pas déjà le signe d'une métamorphose du temps social? En tant que source des gains des marchands et des rémunérations des artisans et des ouvriers des différents métiers, le temps de travail va constituer un terrain d'affrontement et de lutte, mais aussi un lieu d'intervention de l'État pour tenter de réglementer le cadre temporel des activités professionnelles.

\subsection{Le temps des marchandises: une autre manière de voir le temps au XIXe siècle}

Le temps naturel cyclique, mais aussi le temps linéaire et continu de la religion ont été progressivement doublés au Moyen Age d'un autre temps, celui des marchands. Le temps de leurs activités professionnelles apparaît comme le temps du gain, à côté du temps du salut ou du temps météorologique. Cette pression du temps, qui métamorphose les autres temps sociaux, renvoie à cette conception différente de temps abstrait, discontinu et mesurable (op. cité). Au total avec le temps des marchands le temps devient le cadre des activités professionnelles. Le temps est la mesure des activités de travail et finalement des gains des marchands, des rémunérations des artisans et des ouvriers. Pour Le Goff (1977, p. 76) le Moyen Age est bien la période d'apparition, «d'une nouvelle conception du temps, d'un temps qui n'est plus une essence, mais une forme conceptuelle, au service de l'esprit qui en use selon ses besoins, peut le diviser le mesurer - un temps discontinu ». 
Il faut attendre le développement de la société industrielle pour que le rapport entre le travail et le temps prenne un sens un peu différent: désormais, le temps de travail va devenir un temps dominant, ce qui n'exclut pas que d'autres temps continuent à rythmer certaines activités. Ce rapport se caractérise par le fait que « le travail est réglé par le temps » (Thompson, 1967, cité par Debouzy, p. 198), ou encore que «le temps prévaut sur la tâche » (Naville, 1972) ; alors que dans la période préindustrielle c'est plutôt la tâche qui prévaut sur le temps : le travail est réalisé dans le cadre de rythmes habituellement marqués par le temps naturel. Bien entendu, cette opposition entre société agraire et société industrielle n'exclut pas que le temps de l'église continue à rythmer en partie les activités et les fêtes par les offices, les prières et les célébrations, mais aussi par le repos dominical. De même cette émergence du temps de travail mesuré et pressé n'exclut pas que, pour certaines catégories comme les paysans, le temps naturel continue à rythmer leurs activités. Le temps des paysans caractérisé par Grossin (1994), circonscrit leur travail d'une certaine manière : «Il s'effectuait sans hâte, du lever du jour à la tombée de la nuit, dans un cadre familial où hommes et femmes adultes, enfants et vieillards jouaient un rôle dans la mesure de leur force. »

\section{I.2.A. Les trois composantes du temps de travail : durées, horaires et rythmes}

Le cadre temporel qui se met en place au XIX ${ }^{\mathrm{e}}$ siècle est bien celui qui apparaît au Moyen Age pour encadrer les activités professionnelles. On pourrait dire qu'il se généralise, car il s'applique désormais à tous les travailleurs salariés ; la nouveauté réside dans le fait qu'il devient un principe d'organisation des activités de travail. De façon synthétique, Grossin (1994) dans son article intitulé «Les temps de travail» entend par temps de travail «la mesure par l'horloge, d'une activité définie et payée. Il s'agit d'une durée consacrée à la production de biens ou à des services, délimitée, par un horaire. A cette durée succède un temps hors travail ». Mais selon cet auteur le temps de travail est aussi une manière de déposséder l'individu de son temps et de lui imposer un rythme. Le temps devient alors, dans cette conception, un moyen pour organiser les activités qui se déroulent dans le cadre temporel.

(1) Le temps de travail comme durée

Le temps de travail délimite d'abord la durée de la prestation de travail : cette durée définit pour le travailleur, le temps qu'il va passer dans l'entreprise. Au XIX ${ }^{\mathrm{e}}$ siècle et à la différence des siècles précédents, les durées sont extrêmement longues. L'historien 
Fridenson (1993) note qu'au XIX ${ }^{\mathrm{e}}$ siècle, « l'année de travail dans l'industrie atteint vers 1840, de 3000 à 3600 heures. Si elle est tombée à 1 650-2 000 heures, ce n'est assurément pas du fait des employeurs ». Il n'est pas facile d'avoir une idée précise de la durée hebdomadaire moyenne : Debouzy (1979) observe que dans les usines de l'est des EtatsUnis, elle est de 73 heures de 1820 à 1830. En revanche, les données sur la durée journalière sont plus abondantes : les premières études dans le textile aux Etats-Unis font état de 12 h à 12 h 30 (Debouzy, 1979). En France, les durées sont très souvent dénoncées pour appuyer la revendication d'une baisse de la durée journalière et l'établissement de lois pour limiter ces durées. Les témoignages sont accablants : dans l'Isère et dans la Savoie, l'inspecteur du travail rapporte que vers 1874 « la jeunesse commence ordinairement à cinq heures du matin pour finir à 8 heures ou huit heures et demie du soir avec deux heures attribuées au repos » (cité par Grossin, 1992, p. 21).

(2) Le temps de travail comme localisation des périodes de travail : travail de nuit et travail du dimanche

Certes, une heure de travail est, dans une conception abstraite du temps, toujours égale à une heure. Pour le salarié, cette heure prend des significations différentes selon qu'elle se situe le jour ou la nuit, en semaine ou le dimanche. La localisation des périodes de travail est la manière de définir les horaires de travail, c'est-à-dire de répartir les périodes de travail sur la journée, la semaine et le mois : le travail de nuit et le travail du dimanche constitueront les deux points de controverse du Moyen Age, à nos jours. Déjà au Moyen Age, le travail de nuit était pratiqué dans certains métiers. Mais au XIX ${ }^{\mathrm{e}}$ siècle, il n'est pas rare que les journées de travail se prolongent la nuit, comme en témoignent les rapports établis après 1874 par les inspecteurs du travail de la région de Toulouse (cité par Grossin, 1992). «Dans certaines périodes, les patrons, au lieu d'augmenter leur personnel, exigent un surcroît de travail de leur personnel ancien. Pendant ces périodes, la durée du travail est parfois de quatorze, quinze et même seize heures. Les ouvriers ne peuvent s'y soustraire sous peine d'expulsion pendant la bonne saison. Parfois même, l'ouvrier passe la nuit complète du samedi. Il se retire le dimanche après avoir travaillé 24 heures consécutives. Ces procédés sont employés souvent dans les filatures, les fabriques de draps, de tricots, de chapeaux, de chaussures, dans l'industrie des laines et dans certains grands ateliers de Toulouse $\gg$. 
(3) Le temps de travail comme rythme de travail: « la mise en œuvre de la force de travail »

Les deux composantes du temps de travail en tant que durée et en tant qu'horaire peuvent être considérées comme le cadre temporel des activités : ce cadre externe aux activités ne préjuge en rien de la nature et du déroulement de ces activités, donc de l'aspect interne. C'est pourquoi Grossin (1994, p. 131) distingue dans le temps de travail, le contenant et le contenu, c'est-à-dire « d'une part, des aspects externes morphologiques ou géométriques comme la durée et l'horaire de travail, les répartitions des jours ouvrés dans la semaine et/ou l'année et d'autre part, des aspects internes ou constitutifs qui se rapportent à la mise en œuvre de la force de travail : normes, rythmes, séquences - séries de gestes à reproduire -, cadences, distributions programmées des pauses ».

Cette troisième composante du temps de travail est généralement dissociée du cadre temporel et on a aujourd'hui l'habitude de séparer dans l'analyse ce qui relève d'un côté des durées et des horaires de travail et d'un autre côté le rythme. Or s'il y a bien une nouveauté, au regard du cadre temporel du Moyen Age, c'est bien d'utiliser le temps comme moyen d'agir sur la mise en œuvre de la force de travail. Le temps industriel, c'est premièrement, le rassemblement en un même lieu - l'usine - de travailleurs qui vont opérer en même temps selon le rythme des machines. Pour bon nombre d'auteurs, ce rassemblement qui retranche le travailleur du milieu familial est exigé par les machines qui pour fonctionner requièrent la présence humaine. Par voie de conséquence, le temps du travail humain est subordonné au temps des machines, qu'il s'agit d'utiliser le plus longtemps possible ; car comme le fait remarquer Grossin (1994, p. 129), abandonner une machine à vapeur pour un ouvrier d'usine c'est immobiliser un capital d'importance, tandis qu'abandonner une pioche n'a que peu de conséquences économiques. Il ajoute. Cette primauté des rythmes des machines est peut-être une des inventions les plus spectaculaires de la révolution industrielle. Engels $(1874,1976)$ avait déjà indiqué que l'organisation temporelle de la vie sociale, en particulier celle de la vie au travail, dans l'industrie moderne, nécessite des formes de coordination des activités relativement indépendantes des rythmes individuels.

«Tous ces ouvriers, hommes, femmes et enfants sont contraints de commencer et de terminer leur travail à une heure qui est fixée par l'autorité de la vapeur, qui ne s'occupe pas de l'autonomie individuelle». (Engels, 1874, 1976, p. 56) ${ }^{2}$. 
Donc, deuxièmement, le temps industriel subordonne le travail humain au temps des machines, favorise leur synchronisation, mais aussi l'accélération du rythme de travail par une augmentation des cadences. Dans le bilan des travaux qu'elle établit sur « le temps industriel aux Etats-Unis au début du XIXe siècle », Debouzy (1979, p. 204) montre que les métiers mécaniques étaient «accélérés»: «Néanmoins, la synchronisation des opérations impliquait l'asservissement à un rythme de travail régulier et monotone et les contremaîtres (overseers) étaient constamment incités par des primes à accélérer ce rythme. Dès les années 1840, il semble qu'il y ait eu une accélération sensible des cadences et que les ouvrières aient eu à faire fonctionner un nombre croissant de métiers, ce qui ne laissait plus subsister aucun temps mort. »

En résumé, le temps de travail évolue en fonction des pratiques et des manières de voir le temps de travail. S'il constitue depuis le Moyen Age le cadre des activités professionnelles, il n'acquiert toute sa signification contemporaine qu'avec le temps de travail des sociétés industrielles, et plus particulièrement au $\mathrm{XIX}^{\mathrm{e}}$ siècle, il devient le cadre et le principe d'organisation des activités professionnelles. Le temps de travail encadre les activités professionnelles et constitue un moyen pour agir sur la mise en œuvre de la force de travail. C'est bien ce second aspect qu'il s'agit d'expliciter pour caractériser la vision dominante du temps de travail qui se forge au XIX ${ }^{\mathrm{e}}$ siècle.

\section{I.2.B. Caractérisation du temps comme marchandise : subordination et rationalisation}

(1) Le temps de travail comme marchandise : le temps de la subordination

Le travail, c'est avant tout une marchandise qui est l'objet de la négociation entre l'employeur et le salarié. Comme le souligne Supiot (1994, p. 7), « le travailleur est celui qui loue ses services à autrui ; le travail est objet de ce négoce et le marché du travail le lieu de cette négociation. » Si le travailleur a «vendu en toute liberté sa force de travail à un employeur », il reçoit en échange un salaire ; cet échange est un contrat qui fonde les obligations des parties en présence dans cette relation de travail. Mais ne s'agit-il que de payer un salaire contre l'accomplissement d'un travail ? L'employeur a, au fond, acheté du temps et en même temps la possibilité de le gérer à sa guise : l'employeur détient le pouvoir réglementaire. La relation salariale n'est-elle pas la maîtrise temporelle acquise par l'employeur de gérer l'emploi du temps et les accomplissements du salarié ? De manière symétrique, l'institutionnalisation par le contrat de travail, du temps de travail 
comme temps hétéronome, n'implique-t-elle pas la perte de toute maîtrise du temps de travail par le salarié ?

La notion de temps de travail désigne bien la période durant laquelle le travailleur est à la disposition de l'employeur et inversement pour la période de repos. Pour les juristes, « le temps de travail permet d'une part de limiter l'emprise patronale sur la vie du salarié et d'autre part d'évaluer la prestation de ce dernier » (Supiot, 1995, p. 947). Ce temps de travail mesure la durée de l'exécution du contrat ou de réalisation de la prestation de service. Dans l'approche contractuelle de la relation de travail, le temps de travail, c'est le temps de la subordination. Remarquons avec Supiot (1995) que cette codification juridique institutionnalise une coupure entre la période du travail et la période du hors travail, la première période étant définie par l'existence de l'employeur donc d'un contrat : et dans les périodes hors travail rémunéré peuvent se loger des activités contraintes domestiques ou paraprofessionnelles. De plus cette codification juridique est silencieuse sur l'évaluation de la prestation de service qui doit être réalisée suivant le contrat.

Ce temps de la subordination rythme le travail des personnes, leur impose des horaires communs : le temps encadre la prestation et peut être interprété comme une «discipline temporelle ». La ponctualité dans le travail et la régularité de la présence constituent les synchroniseurs de la vie d'une personne qui travaille. Cette discipline du temps ne s'arrête pas aux portes de l'usine que l'ouvrier a pu regagner: elle se poursuit dans l'atelier où le temps des machines et le temps des travailleurs sont synchronisés. Ce temps de travail abstrait et quantitatif, mesuré et tronçonné est géré par l'employeur qui en a désormais la maîtrise. Le temps de l'ouvrier est enfermé dans le règlement.

(2) Le temps de travail et l'économie de temps : le temps de la rationalisation

Avec la mécanisation, le temps de travail a un coût élevé. Dès lors que le temps, devient une marchandise, l'économie de temps devient primordiale. Rappelons que pour Marx $(1867,1967)$ l'économie de temps constitue la première loi de l'économie capitaliste. Le développement de l'industrie fait du temps de travail une règle d'organisation des activités de travail : pour l'employeur, il s'agit de rationaliser le temps de présence du salarié, qu'il a acheté. Pour ne rien perdre de ce temps qu'il a acheté, et lorsqu'il atteint les limites de l'allongement de la journée de travail, il ne reste plus à l'employeur qu'à intensifier le travail. En d'autres mots, rationaliser ce n'est pas travailler plus, mais c'est travailler autrement : c'est la chasse aux temps morts, à la limitation des pauses, etc. La notion de 
flexibilité temporelle ne constitue-t-elle pas la manière contemporaine de procéder à cette rationalisation? Retenons que, dans la perspective marxienne, le temps prend comme le souligne Alaluf (1986), « une importance décisive dans la compréhension des structures sociales ».

Il apparaît clairement qu'avec le XIX ${ }^{\mathrm{e}}$ siècle le temps de travail, c'est le temps « du gain » pour les uns et le temps des «rémunérations» pour les autres. Le temps de travail représente la base de calcul pour la détermination des salaires des personnes qui travaillent. On comprendra aisément que ce temps de travail constitue un enjeu des plus serrés, pour les employeurs comme pour les salariés : temps de travail et salaire sont des terrains d'affrontement privilégiés. Par voie de conséquence, toute réglementation du temps de travail doit aussi s'attaquer et résoudre à la question des salaires : nous avons déjà noté qu'à une certaine époque, où il est vrai la pression temporelle est moyenne (si on en juge par le fait que le nombre de jours travaillés par an est de 250 pour les $\mathrm{XV}^{\mathrm{e}}, \mathrm{XVI}^{\mathrm{e}}$ et $\mathrm{XVII}^{\mathrm{e}}$ siècles, d'après Grossin, 1994) les journaliers réclamaient un allongement de la durée du travail pour augmenter leur salaire. En effet, la réglementation ne protège pas seulement le salarié contre son employeur, mais aussi contre lui-même.

Cette relation entre le temps de travail et le salaire sera utilisée par certains contre la diminution de la durée du travail : diminuer le temps de travail, c'est en quelque sorte réduire les salaires. Cet argument est particulièrement développé au XIX ${ }^{\mathrm{e}}$ siècle pour lutter contre l'apparition des lois touchant la réduction; Barthes, sénateur des Basses-Pyrénées déclare à la Chambre le 21 mars 1882 : « ce n'est pas la peine de réduire la durée du travail pour ensuite accorder des heures supplémentaires, recherchées par les ouvriers et devenues la règle » (cité par Grossin, 1992, p. 76).

\section{I.2.C. Temps monochrone et ethos de la besogne}

Déjà, le temps des marchands repose sur une conception du temps abstrait, discontinu et mesurable comme le disait Le Goff (1977, p. 76). Le temps des marchandises veut dire que le temps de travail mesure la durée de la réalisation du contrat entre l'employeur et le salarié. En tant que durée de subordination et instrument de rationalisation, le temps repose sur une conception du temps monochrone et sur une éthique de la besogne. 
(1) Il s'agit d'un temps monochrone et gouverné extérieurement

Il dépossède le travailleur de la maîtrise de son temps. Ce à quoi vont s'opposer de nombreux auteurs tel Proudhon qui imagine une communauté qui gérerait elle-même sa temporalité et il refuse "l'idée d'une cohérence qui découlerait d'un rythme obligatoire venu d'une constance transcendante, qu'elle soit économique (le capital), politique (l'État) ou idéologique (l'Absolu religieux)» (Ansart, 1991, p. 239). Ce temps « monochrone » se caractérise par une planification des horaires et des programmes auxquels les individus doivent se conformer : «le temps monochrone correspond au temps linéaire, quantitatif, mesuré de façon très précise de la civilisation industrielle, tandis que le temps polychrone est indéterminé et correspond à des réalités culturelles très variées » (Gasparini, 1990, p. 733).

(2) Il s'agit d'une adhésion à « l'ethos de la besogne»

Dès lors que le temps de travail est le temps du gain, il suppose une adhésion à ce que Weber appellera l'ethos de la besogne. Il ne faut pas perdre de temps, il faut épargner son temps et l'utiliser avec le maximum de zèle pour son travail.

Weber $(1905,1964)$ a montré que la quantification du temps de travail sous forme d'une durée mesurable en heures journalières suppose un bouleversement de l'organisation de la production $^{3}$; plus largement, il montre que la quantification du temps correspond à un changement de rationalité, à un bouleversement de l'organisation de la vie sociale qui s'opère conjointement avec l'apparition de nouvelles représentations du temps promu par la Réforme et ses principaux représentants (Luther et Calvin). «Le temps est précieux, infiniment, car chaque heure perdue est soustraite au travail qui concourt à la gloire divine », Weber (1905, 1964, p. 189).

Weber (1905, 1964, p. 69) décrit le passage difficile d'un Ethos traditionnel du travail à un Ethos nouveau du travail industriel sous l'influence du protestantisme en reprenant le cas de l'industrie textile qui garde jusqu'à la fin du $\mathrm{XIX}^{\mathrm{e}}$ siècle, malgré sa forme d'organisation capitaliste, un Ethos traditionnel déterminant pour la journée du travail : « le nombre d'heures de travail était très modéré, cinq à six par jour, parfois beaucoup moins, davantage le cas échéant, dans les moments de presse. Les gains étaient modestes ; suffisants pour mener une vie décente et mettre de l'argent de côté dans les bonnes années. Dans l'ensemble, les concurrents entretenaient entre eux de bonnes relations, étant 
d'accord sur les principes essentiels des opérations. Une visite prolongée au café, chaque jour, un petit cercle d'amis - la vie agréable et tranquille ».

Néanmoins d'un coup, cet Ethos s'effondre sous la pression de la concurrence et par de nouvelles façons de vivre le temps. «L'ancien mode de vie, confortable et sans façon, lâchait pied devant la sobriété de quelques-uns » (op. cit., p. 70). L'éthique protestante élève le travail au rang d'une valeur spirituelle universelle en soi. Un ethos bourgeois de la besogne exerce un contrôle méthodique sur les conduites et se réalise dans « l'ascétisme » qui définit une mise en forme rationnelle de l'existence tout entière, rapportée à la volonté de dieu (Weber, 1905, 1964, p. 184).

C'est d'ailleurs d'abord la bourgeoisie d'affaires qui a augmenté sa durée de travail à partir du $\mathrm{XVI}^{\mathrm{e}}$ siècle traduisant ainsi leur adhésion à l'ethos de la besogne. Les durées du travail des autres catégories socioprofessionnelles restaient à peu près stables entre 10 et 13 heures par jour (Découflé \& Svendson, 1984, p. 60).

\section{LA CONSTRUCTION DE L'ORDRE TEMPOREL PAR LES REgLES : LA NORME TEMPORELLE}

Le temps de travail est une norme composée d'un ensemble de règles qui vont orienter les comportements : ces règles ont un objet qui est la protection du travailleur dont « le corps fait irruption dans l'ordre juridique », comme l'indique Supiot (1994); en tant que notre norme, elle oblige en quelque sorte les individus à s'y ranger : les règles touchant le temps de travail vont constituer un dispositif de contraintes qui vont peser sur les individus. Quelles sont ces règles ? Quelle et leur valeur coercitive ? Par qui sont-elles élaborées ? Notre hypothèse met l'accent sur la nécessité d'expliciter la contrainte temporelle et elle oblige à caractériser les règles temporelles, leur nature, leur mode d'élaboration, leurs effets. En effet, comprendre l'ordre temporel dominant implique donc faire le détour par les dispositifs qui règlent les actions de travail : nous examinerons tout d'abord la règle fondatrice de la protection ouvrière de 1841 consécutive au rapport de Villermé (1840, 1989) puis, nous présenterons les différents dispositifs qui traduisent cette lente montée de la réglementation.

\section{II.1. Le corps fait irruption dans l'ordre juridique}

Avant d'aborder la question de la réglementation dans le travail, il nous faut revenir brièvement sur les origines de la réglementation. Si le droit du travail «a pour objet 
essentiel d'encadrer l'exercice du pouvoir qu'il confère ainsi à une personne sur une autre » (Supiot, 1994, p. 110), c'est bien qu'il repose sur une certaine manière de voir le travail non comme une sphère pacifiée, "mais comme une relation où l'on peut commander et où l'autre doit obéir ». Le pouvoir, et donc son encadrement, est bien au cœur de la relation de travail dans la mesure où la subordination du travailleur caractérise le contrat qui le lie à son employeur.

Autrement dit, cette mise en forme d'un corps de règles applicable dans le travail prend son essor avec la conception moderne du travail comme marchandise. Dans cette conception abstraite du travail, «le travailleur est celui qui loue ses services à autrui, le travail est l'objet de ce négoce et le marché du travail le lieu de cette négociation » (Supiot, 1994, p. 7). Bien entendu le travail ainsi défini, ne désigne pas toutes les formes de travail, mais simplement les activités professionnelles qui font l'objet d'une négociation instituée dans un contrat. Le décret d'Allarde du 16 février 1791 précise bien «qu'il sera libre à toute personne de faire tel négoce, d'exercer telle profession, art ou métier que bon lui semble». Le travail comme bien négociable fait l'objet d'un contrat qui précise les obligations de chacune des parties et en particulier le paiement du salaire contre l'accomplissement d'un travail. Quel est donc l'objet de ce contrat? La relation de travail met en jeu les accomplissements qui font l'objet d'une formulation des règles : celles-ci concernent l'organisation des accomplissements et donc le travailleur et sa capacité de travail. D'où un ensemble de règles qui vont préciser le comportement du salarié, ses obligations mais aussi les contraintes qui vont peser sur la personne qui travaille. La règle de travail que le droit du travail formule, tire ses origines de la sécurité physique des personnes, car « dans la relation de travail, le travailleur, à la différence de l'employeur [...] risque sa peau. Et c'est d'abord pour sauver cette dernière que le droit du travail s'est constitué » (Supiot, 1994, p. 68). Ce droit à l'intégrité physique s'est affirmé au niveau international comme en témoigne la directive 93-391 du 12 juin 1989 de l'Organisation Internationale du Travail (OIT).

\section{II.1.A. Nécessité d'une loi pour protéger la santé selon Villermé.}

Dire que le travail dans sa conception moderne c'est-à-dire comme marchandise s'accompagne de la formulation de règles concernant la protection des salariés pourrait laisser croire que la réglementation va de soi et qu'il suffirait de quelques «bonnes volontés » pour qu'une réglementation protectrice apparaisse. Un bref retour en arrière sur l'apparition des mesures protectrices est particulièrement éclairant. La première règle de 
protection date de 1841 en France : il s'agit de la loi fixant à 8 h par jour la durée du travail des enfants de 8 à 12 ans et à 12 h par jour celle des enfants de 12 à 16 ans (Guedj \& Vindt, 1997, p. 33). De telles mesures avaient déjà été prises en Grande Bretagne en 1819 interdisant le travail aux enfants de moins de 9 ans. Il ne s'agit pas ici de superposer les diverses mesures protectrices pour montrer l'importance au $\mathrm{XIX}^{\mathrm{e}}$ siècle des règles de sécurité. Il s'agit de rappeler la dynamique sociale, les tensions et les rapports de force qu'il faut instaurer pour formuler une règle de droit applicable à tous. Cette dynamique de construction de la règle de protection se caractérise en France premièrement par ce qu'on pourrait appeler un rapport d'expert, celui de Louis-René Villermé publié en 1840. De quoi s'agit-il ? D'une vaste enquête sur la classe ouvrière dans les manufactures constatant dans le chapitre III que la durée journalière est très longue. «Cette durée est très longue, excepté dans les temps de crise : la journée est ordinairement, pour tous les ouvriers employés dans les ateliers des manufactures de coton et de laine, de quinze heures à quinze heures et demie, sur lesquelles on en exige treize de travail effectif » (Villermé, 1840, 1989, p. 411). L'auteur précise que dans la ville de Saint Quentin, «les ouvriers de tout âge et de tout sexe (souligné par l'auteur) travaillent treize heures par jour ». Il ajoute que « dans les temps de grande commande ou de grande presse, le travail des manufactures se prolonge quelquefois toute la nuit du samedi au dimanche ».

Le chapitre IV de ce rapport est consacré aux « enfants employés dans les manufactures ». Villermé (1840, 1989, p. 426 et suivantes) constate l'aspect effrayant de la condition des enfants dans les manufactures dont voici quelques extraits : «En général le travail de nuit et, pour les enfants, une cause de grande démoralisation (souligné par l'auteur) [...] Les renseignements s'accordent tous au reste pour établir que l'instruction des enfants qui ont été admis dans les ateliers dès l'âge de six ans est nulle et qu'ordinairement ceux qui sont reçus avant dix ou onze ans, ne savent ni lire ni écrire ». S'appuyant sur le rapport du bureau des manufactures, Villermé $(1840,1989)$ indique que les enfants de 9 ans peuvent être admis à travailler.

Et Villermé (1840, 1989, pp. 416-417) d'appeler de tous ses vœux la promulgation d'une loi pour protéger les enfants: «Le remède au dépérissement des enfants dans les manufactures, à l'abus homicide qu'on en fait, ne saurait donc se trouver que dans une loi ou au règlement qui fixerait, d'après l'âge de ces ouvriers, un maximum (souligné par l'auteur) à la durée journalière du travail [...]. Mais on comprend si bien, dans cette Société, quelle est la principale cause du mal, qu'on y a plus d'une fois exprimé le besoin 
d'une loi qui limiterait comme en Angleterre, la durée trop longue du travail et ne permettrait plus de le prolonger pendant la nuit. »

\section{II.1.B. Députés et sénateurs s'entendent en 1839 pour éviter une loi qui limiterait la durée du travail}

Pourtant, cette loi fera l'objet de nombreuses discussions à la Chambre des Pairs lors de sa séance du 31 mai 1839, comme à la Chambre des députés lors de sa séance du 15 juin 1839. L'actualité des propos tenus, comme leur pertinence au regard de la construction des règles de travail, nous invite à les retracer succinctement. A la Chambre des Pairs, le rapporteur précise que le gouvernement est « disposé » à faire une loi en la matière, mais qu'il y a de nombreuses difficultés : «L'extrême difficulté est de trouver un plus grand nombre d'enfants, afin d'en substituer quelques-uns à ceux dont on perdrait le temps de travail » (Villermé, 1840, 1989, p. 598). En effet, à la Chambre des députés, le rapporteur ne manque pas de rappeler les conditions catastrophiques dans lesquelles se trouvent les enfants admis en atelier à l'âge de 8 ans, « et quelquefois depuis l'âge de 6 ans ; que leurs forces corporelles n'ont point encore à cette époque de la vie, acquis un développement suffisant pour le travail auquel ils sont assujettis ; que cependant on les retient quinze et quelquefois dix-sept heures par jour, fixées à la même occupation, en ne leur laissant qu'une heure et demie de repos pour prendre leur nourriture ; que se trouvant privés à un âge aussi tendre de toute culture intellectuelle et morale, ils végètent sans instruction, privés des affections qui auraient pu se développer dans leur famille, étrangers aux sentiments religieux, et à tout ce qui constitue la dignité de l'homme » (Villermé, 1840, 1989, p. 606).

A ce rapport l'un des députés M. Falchiron, répond qu'une loi fixant une durée est impossible car tout est variable. «On ne pourrait pas, dit-il, faire une loi qui fixerait définitivement l'âge où les enfants doivent entrer dans les ateliers et le temps d'occupation de leur part ». Pour lui il faudrait faire varier la durée du travail en fonction du contexte, car « ce qui conviendra pour le midi, ne conviendra pas au nord, et ce qu'on fera pour le nord sera mauvais pour le midi ».

N'est-on pas là devant le débat qui se poursuit aujourd'hui sur la pertinence d'une règle juridique? N'a-t-on pas là tous les ingrédients concernant la production des règles ? 


\section{II.2. La lente montée de la réglementation sur le temps de travail}

Avant de donner les grands traits de cette réglementation, il convient d'observer tout d'abord que ces règles se développent en relation avec le contexte et la manière de voir le temps de travail. La pression sociale et les conflits ont sans aucun doute eu leur rôle dans la production de règles protectrices. Ensuite, il convient de remarquer qu'une fois déposé auprès des parlementaires, un projet de loi peut attendre 10 ans avant d'être voté, après avoir subi des amendements qui souvent, lui font perdre sa substance. Enfin, une loi n'est pas grand-chose, si la puissance publique n'est pas dotée de moyens pour la faire respecter il faut bien préciser que toutes ces mesures sont généralement perçues par les employeurs comme une remise en cause de leurs prérogatives susceptibles de mettre en danger l'efficacité de leur entreprise.

L'importance et le poids du contexte, l'intervention des parlementaires et les forces coercitives associées à la loi constituent des éléments capitaux pour apprécier la signification des lois. Notre objectif ici est de caractériser les étapes et la nature de cette réglementation du temps de travail, afin d'expliciter le creuset de ce que nous appellerons, les normes temporelles.

\section{II.2.A. L'allongement de la durée du travail et la première loi de 1841 : l'intervention de l'État}

$\mathrm{Au}$ Moyen Age comme du XVe au XVII ${ }^{\mathrm{e}}$ siècle la pression temporelle est en moyenne faible : le nombre de jours chômés et de fêtes religieuses est important. Fridenson (1993, p. 20) évalue à 200 jours le nombre de jours travaillés au XVII ${ }^{\mathrm{e}}$ siècle, tandis que Grossin (1992) parle pour les XVe, XVI et $\mathrm{XVII}^{\mathrm{e}}$ siècles de 230 jours de travail. En revanche, les auteurs s'accordent pour noter les variations au cours de l'année des durées journalières ; en partant des 164 jours de fêtes du XVII ${ }^{\mathrm{e}}$ siècle

Les règles des métiers disparaissent avec l'interdiction des corporations par la loi Le Chapelier du 14 juin 1791. Ce sont des règlements de police qui déterminent le temps de travail des ouvriers du bâtiment, tailleurs de pierre, carreleurs, menuisiers, serruriers, etc. Avec la loi Le Chapelier de 1791 les employeurs obtiennent carte blanche : " La loi leur reconnaît plein pouvoir sur le temps de travail de leurs salariés. Parallèlement, ils s'engagent dans un combat très long pour éradiquer les usages ouvriers qui rendent la semaine de travail très poreuse : l'absence du lundi suivant la paye (la Saint Lundi), le départ de bonne heure le samedi durant l'été, le travail à temps partiel pendant le temps des 
récoltes, les pauses tout au long de la journée » (Fridenson, 1993, p. 20). Et l'auteur indique l'engagement des employeurs pour augmenter la durée journalière du travail et pour diminuer le nombre de jours chômés.

La loi du 22 mars 1841 est la première mesure réglementant la durée du travail dans l'industrie : elle concerne une catégorie de main-d'œuvre, les enfants employés dans les manufactures les usines ou les ateliers. Désormais, les enfants de moins de 12 ans ne devront travailler pas plus de 8 heures par jour et le travail de nuit leur est interdit jusqu'à 13 ans. En effet, le commentaire de Fridenson (1993, p. 21) est de grande importance, car, en plus des conditions lamentables d'emploi de ces enfants, dénoncées par Villermé dès la fin des années 30, c'est en fait pour limiter la concurrence qu'elle a été mise en place : «Les industriels du textile de Mulhouse qui l'ont réclamée depuis 1827 y voient un moyen d'éviter une concurrence mineure en imposant un standard uniforme de durée du travail des jeunes. Mais pour des raisons pratiques, elle est en fait restée lettre morte pendant longtemps. »

\section{II.2.B. Un demi-siècle pour aboutir aux 10 heures par jour (1841-1904) : des inspecteurs pour faire respecter la loi}

La loi du 9 septembre 1848, sur les douze heures par jour pour tous, constitue la deuxième réglementation de la durée du travail. Là encore, il faut faire deux observations que nous empruntons à Grossin (1992, p. 242). La première indique qu'en fait ce qui était demandé c'était une diminution plus importante : 10 heures pour Paris alors qu'elle était de 11 heures et 11 heures pour la province où elle était de 12 heures. C'est ce qui avait été voté dans l'élan révolutionnaire le 2 mars 1848 et abrogé par la constituant le 9 septembre 1848. La deuxième remarque concerne les dépassements inscrits dans la loi dans son article 2 qui les prévoit «à raison de la nature des industries ou des causes de force majeure $»:$ le principe des heures supplémentaires est acquis.

En effet, cette loi en elle-même n'apporte pas de grande nouveauté sur la durée du travail, si ce n'est qu'elle constitue un pas vers la réduction de la durée journalière et qu'elle annonce le cadre hebdomadaire, comme référentiel pour comptabiliser les heures de travail. On constatera que les composantes principales du temps de travail se trouvent énoncées : journée, semaine, repos. Sa nouveauté réside dans le fait qu'elle précise les moyens pour faire respecter la loi. Désormais, « les inspecteurs du travail sont chargés d'assurer l'exécution de la présente loi et de la loi du 9 septembre $1848 »$ (article 17). L'importance de cette loi n'est pas totalement dans son résultat, mais dans les discussions 
qui ont précédé son élaboration. Il aura fallu 13 ans pour que le projet de loi limitant la durée du travail à 10 heures soit envisagé.

La loi des 10 heures par jour pour tous : 30 mars 1900 loi parachève la précédente, puisqu'elle généralise les 10 heures pour tous. Mais elle institue cette journée de 10 heures par paliers successifs et « cela signifie que la journée de 10 heures n'est effective que le $1^{\mathrm{er}}$ avril $1904 »$.

\section{II.2.C. La norme temporelle pour tous $(1900-1980): 40$ heures par semaine, 8 h par jour, repos de fin de semaine et les congés payés}

Le repos hebdomadaire du dimanche est fixé par la loi de 1906. Mais là encore cette loi sera retravaillée jusqu'en 1926. Le code du travail stipule néanmoins «que certaines dérogations peuvent intervenir, dans certains cas, pour un repos hebdomadaire un autre jour que le dimanche » (art. L. 221-2, cité par Grossin, 1992, p. 249).

La journée de 8 heures du 23 avril 1919 constitue un mot d'ordre syndical dès 1888. Mais son application sera progressive et sectorielle. Cette loi a été obtenue au lendemain de la première guerre mondiale, à l'issue du conflit qui amenait le gouvernement de Clémenceau à céder pour éviter l'épreuve de force (Fridenson, 1993, p. 25).

Les grèves avec occupation d'usine de mai-juin 1936 débouchent sur deux lois (Fridenson, 1993, p. 26) : « la première instaure la semaine des quarante heures sans perte de salaire, ce que demandait la CGT». La seconde loi introduit les congés payés : le 21 juin 1936.

Les lois Auroux ramènent en 1982 la durée hebdomadaire de 40 à 39 heures et instaurent la $5^{\mathrm{e}}$ semaine de congés.

En résumé, la réglementation touchant le temps de travail contribue à définir la norme temporelle ; elle s'échelonne en gros sur 100 ans selon la même logique : de 1841 à 1936, il s'agit (a) de réduire la durée journalière du travail, qui passe de plus de 13 heures à 8 heures, (b) de réduire la durée hebdomadaire et d'instaurer le repos hebdomadaire d'une journée en 1906 à 2 jours en 1936. Enfin (c) il s'agit d'instaurer les congés payés qui comportent 2 semaines en 1936. Nous avons présenté cette constitution de la norme temporelle en trois grands moments : le premier (de 1830 à 1841) consacre l'intervention de l'État dans le domaine de la relation de l'employeur aux salariés; le second (18411904) consacre les inspecteurs comme l'acteur chargé de faire respecter la loi et de sanctionner. Le troisième (1900-1980), consacre la norme temporelle pour tous avec une 
durée hebdomadaire stable, une durée journalière fixe, 2 jours de repos consécutifs, enfin 5 semaines de congés. Telles sont les principales composantes de l'ordre temporel :

- une norme (durée hebdomadaire, journalière, repos, congés),

- l'intervention de l'État comme puissance normative,

- la règle commune crée le collectif des destinataires,

- un dispositif de sanction extrêmement développé.

On ne peut comprendre l'ordre temporel qu'en détaillant ces composantes et en explicitant leur contenu juridique et social : c'est ce que nous nous proposons de faire.

\section{II.2.D. La norme temporelle : 150 ans d'histoire}

D'où vient l'ordre temporel ? De cette conjonction entre une conception du temps centrée sur l'encadrement et l'organisation des activités de travail et une norme temporelle sanctionnée par une cascade de règlements visant à encadrer la relation employeur-salarié et à cadrer les activités de travail dans le temps. D'où vient la force de cet ordre temporel ? Il semble, en effet, que la norme temporelle ait pris racine dans les représentations des individus et dans les pratiques. Cette norme paraît incontournable, solidement établie : la première raison provient du fait qu'elle n'a pas été décrétée mécaniquement, mais bien qu'elle se soit construite sur 150 ans, de 1830 à 1980. Nous changeons de norme temporelle à partir des années 80, ce que nous développerons au chapitre suivant (chapitre II).

Notre hypothèse est que la force de la norme temporelle qui s'est constituée autour du travail, tient à la combinaison entre (a) un référentiel temporel stable, articulant la vie professionnelle et la vie non professionnelle, (b) l'intervention de l'État, (c) la visée de la règle, collective et donc pour tous, (d) enfin le dispositif de sanctions que le corps des inspecteurs viendra mettre en œuvre. En revanche, on ne peut pas comprendre aujourd'hui le débat sur le temps de travail si on fait abstraction de 150 ans d'histoire ou en exemptant les composantes de l'ordre temporel dominant. C'est pourquoi nous proposons d'aborder ces composantes.

\section{II.2.D.1. La norme temporelle : durée du travail, repos, congé}

La norme temporelle correspond à un découpage dans le temps entre les activités professionnelles et les activités non professionnelles : ce découpage est exprimé en durée 
du travail. Parler de découpage, c'est donc mettre en évidence l'enjeu de cette frontière, que les uns et les autres vont tenter de tirer à eux : pour les salariés, il s'agit de diminuer le temps de travail et pour les employeurs il s'agit au contraire de lutter contre cette diminution, voire d'augmenter la durée du travail.

On pourrait caractériser la norme temporelle par ses quatre composantes :

premièrement la durée journalière du travail. C'est la plus ancienne composante du temps de travail qui était exprimée en durée journalière. Sans doute que ce référentiel journalier tire ses origines de l'alternance du jour et de la nuit, donc du temps naturel. N'oublions pas que les premières lois portent sur cette durée journalière: en gros de 1840 à 1900 on légifère sur la durée journalière qui passe de 13 heures à 10 heures par jour. Deuxièmement, il y a la durée hebdomadaire: la semaine devient le référentiel incontournable qui s'est constitué en presque un siècle. En effet, la référence hebdomadaire date de 1892 c'est pourquoi la loi indique la durée hebdomadaire ne peut dépasser 60 heures par semaine. Il faudra attendre 1936 pour que les 40 heures soient institutionnalisées; en même temps se constitue une norme qui devient le référentiel le moins contesté, le cadre d'évaluation des durées du travail, en même temps que le cadre du déroulement temporel du travail. Troisièmement, le repos de fin de semaine constitue non seulement une interruption du travail dont la durée va être âprement disputée, mais un enjeu entre le temps professionnel et le temps de l'église. Le repos hebdomadaire existe depuis le Moyen Age sous forme d'arrêt de travail pour célébrer les fêtes notamment religieuses. En revanche, au $\mathrm{XIX}^{\mathrm{e}}$ siècle ce repos a été compromis et il aura fallu attendre 1906 pour qu'un jour de repos par semaine soit légalisé et 1936 pour que les 2 jours de repos par semaine constituent la norme légale. Enfin quatrièmement, les congés payés constituent la quatrième et dernière composante de la norme temporelle. Elle est récente, puisqu'il faut attendre 1936 pour que la norme des congés payés s'installe avec 2 semaines, pour arriver en 1982 à la $5^{\mathrm{e}}$ semaine de congés : notons qu'il aura fallu 20 ans pour passer de 2 à 3 semaines de congés (de 1936 à 1956).

\section{II.2.D.2. La consécration de la force publique comme puissance normative}

La deuxième caractéristique de la réglementation est de fixer une norme temporelle par le législateur. Depuis la première réglementation de 1841 concernant la protection, ce principe n'a jamais cessé d'être discuté. Rappelons-nous, ce que le Ministre lui-même disait à l'assemblée, quelques mois avant le vote de cette loi, que le gouvernement n'était pas opposé à cette loi, mais que « si on faisait une loi, je demande que cette loi se contentât 
de dire qu'il y aura un temps fixé de travail pour les enfants, selon les localités [...] »; le Ministre du Commerce ajoutait «Je pense qu'il serait plus convenable d'autoriser les conseils généraux à faire des règlements sur cette matière, lesquels appropriés aux localités auraient toute l'efficacité désirable » (cité dans l'ouvrage de Villermé, 1840, 1989, p. 609$610)$.

Cette première argumentation est tournée vers le développement d'une production normative locale, laissant le soin aux locaux de définir, dans le cadre de la loi, les normes pratiques qu'il s'agit de faire respecter. Certains iront jusqu'à refuser l'autorité de l'État dans la mesure où s'exerce celle du père de famille, comme c'est le cas dans les ateliers familiaux.

\section{II.2.D.3. La dimension collective de la règle}

En même temps, le corps de règles juridiques visant à protéger l'ensemble des salariés crée un repère identitaire pour les bénéficiaires de la règle juridique. En même temps que la règle juridique nomme, classe et qualifie des individus, elle définit les pourtours d'un collectif qui possède les mêmes caractéristiques. Cette incidence de la règle juridique sur l'identité collective réside « dans le lien invisible qui unit ceux qui sans être liés par un rapport juridique formel se reconnaissent une même qualification juridique. Ce lien invisible, né du sentiment d'appartenance à une même catégorie juridique, s'identifie absolument avec le lien de fraternité » (Supiot, 1994, p. 83). C'est dire que le droit du travail crée les conditions de solidarité entre ceux qui ont le sentiment d'appartenir au même groupe. Le contrat de travail basé sur une conception abstraite du travail, efface la diversité concrète des travaux et du même coup, « autorise en retour l'apparition d'une identité collective chez ceux qui ont en commun de n'être que des travailleurs ». Cette identité collective est bien le produit du contrat de travail qui regroupe sous une même catégorie juridique (l'employeur ou le salarié) des individus différents : le contrat de travail à durée indéterminée et à temps plein a fortement contribué à créer cette identité collective.

L'insistance sur la dimension collective de la relation de travail n'est pas sans importance, si on considère aujourd'hui les différentes formes d'individualisation qui se mettent en place et qui risquent d'entraîner la réduction des protections de l'individu par le groupe. La communauté de droits et d'intérêts ne risque-t-elle pas de disparaître au profit d'une individualisation de la relation de travail ? L'individualisation n'entraînera-t-elle pas la remise en cause des droits enracinés dans le collectif? La norme du plein emploi et du contrat à temps plein et à durée indéterminée ne sera-t-elle pas remise en cause ? 


\section{II.2.D.4. Les moyens de la loi : l'inspecteur du travail}

Une autre caractéristique des normes temporelles concerne leur force coercitive. S'agissant de normes juridiques, elles sont bien souvent restées lettre morte, faute de disposer de moyens pour les faire appliquer : des hommes d'abord représentant la puissance publique et un dispositif de sanction. Nous reprendrons ici l'essentiel de l'argumentation à Grossin (1992, p. 187). Dès lors qu'à la norme temporelle sont associées des modalités d'intervention pour la faire respecter, elle devient plus impérieuse et donc plus dangereuse pour ceux qui la combattent. C'est par cette troisième caractéristique qu'on peut comprendre la signification de la lenteur et la véhémence dans l'opposition à la venue de certaines lois.

(1) La création des inspecteurs du travail : 15 inspecteurs. Très tôt un « corps d'inspecteurs aux mains du gouvernement » avait été demandé : 4 inspecteurs généraux, ayant chacun sous ses ordres un inspecteur divisionnaire. Mais il faut attendre la loi de 1874 pour que 15 inspecteurs divisionnaires soient placés à la tête des 15 circonscriptions administratives de la France. En effet, à ces 15 inspecteurs la loi a confié aux départements le soin de nommer des inspecteurs (divisionnaires) du travail, rétribués sur leur ressource propre. Il faut attendre 1882 pour que la loi porte le nombre d'inspecteurs divisionnaires de 15 à 21 et on compte à cette date 44 inspecteurs départementaux.

(2) La consécration de l'intervention de l'État dans les usines : un conflit de pouvoir. Les attributions des inspecteurs ne sont clairement spécifiées que lors de la loi de 1892, soit presque 20 ans après leur création. En effet, l'article 17 précise que «Les inspecteurs du travail sont chargés d'assurer l'exécution de la loi de $1848 »$.

L'ordre temporel ne se construit pas seulement avec des conceptions du temps de travail, qui mettent au centre les activités professionnelles. Si le temps devient le temps des affaires, le temps des gains, il est à la fois le cadre des actions et leur principe de leur organisation. Mais il faudra attendre le XIXe siècle pour que des règles juridiques viennent sanctionner l'irruption du temps de travail comme pivot structurant l'ordre temporel. Les dispositifs juridiques qui se mettent en place, se caractérisent, (a) par des règles visant à limiter la durée journalière du travail pour protéger les enfants confrontés à de rudes conditions temporelles de travail. La montée de ces dispositifs se caractérise aussi (b) par un débat d'une grande violence, les uns voulant qu'une loi commune s'applique à tous les enfants, les autres s'opposant à toute limitation de la durée du travail. Certes, ils consacreront l'État comme l'autorité normative, capable de faire des lois et de les faire 
appliquer ; mais ces dispositifs montreront aussi combien les règles temporelles constituent un enjeu relatif à la nature de la norme, mais aussi à l'idée même d'une intervention extérieure dans les entreprises.

\section{LES PRATIQUES}

Le temps de travail est bien le cadre des activités professionnelles et le principe de leur organisation : c'est ce que l'analyse des conceptions du temps de travail et l'analyse des règlements nous montrent. Mais pour préciser ce qu'est l'ordre temporel, il nous reste à nous référer aussi aux pratiques : bien entendu, les pratiques ne constituent pas une copie de cet ordre légal, mais plutôt leur traduction dans des contextes singuliers. Nous allons voir, par exemple, comment le temps des guerres, et plus généralement l'impact d'un contexte de croissance ou de récession, fait varier le temps de travail.

Voilà posé le problème de l'articulation au concret entre des normes juridiques et des règles sociales: de notre point de vue, cette relation ne doit pas être posée dans une perspective déterministe, comme si le droit déterminait les pratiques ; tout au plus la règle juridique influence-t-elle les pratiques. On pourrait même aller plus loin, en précisant que la loi est souvent issue des pratiques, même si comme nous l'avons vu, il existe de nombreux barrages pour qu'une loi ne se mette pas en place.

La durée du travail est la figure emblématique de cette norme temporelle : elle occupe une place centrale pour plusieurs raisons. D'abord, la durée hebdomadaire constitue le référentiel le mieux partagé pour parler du temps de travail : elle est fixée par la loi, objet de luttes sociales. Ensuite, cette durée sert de mesure, de base de calcul (notamment des salaires) et de comparaisons pour le temps de travail, même si, comme nous le verrons, cette base peut être établie selon plusieurs critères. Enfin, la durée du travail, qui s'est établie autour des 40 heures, constitue une référence symbolique, qui trône au milieu de toutes les autres références : elle constitue le principe d'articulation du temps des activités professionnelles et du temps des activités non professionnelles.

Compte tenu de sa position centrale, le temps de travail nous est apparu comme un excellent révélateur de cette combinaison des conceptions et des règlements, dans les pratiques. Nous proposons tout d'abord, de montrer la complexité de cette mesure que constitue la durée du travail : aucune définition ne s'impose pour caractériser la durée du travail ; il faudrait alors expliciter les enjeux. Ensuite, nous monterons l'évolution de la 
durée du travail en France, au cours d'un siècle environ. Enfin, nous tenterons de situer les tendances observées en France, dans le contexte européen.

\section{III.1. La durée du travail de quoi parle-t-on?}

Le temps de travail apparaît comme un univers de règles juridiques et de pratiques qui ne se superposent pas toujours.

La mesure de la durée du travail fait apparaître un premier écart entre la norme juridique et les durées pratiquées : il n’y a pas de correspondance mécanique entre ces deux ordres de réalité; derrière la variabilité des pratiques, se cache des modes de comptages fort différents. Il existe, en effet, une pluralité d'approches pour mesurer la durée du travail. L’importance de l'écart entre la norme juridique et les durées pratiquées, dépend tout d'abord de l'indicateur retenu. Ainsi surgissent des mesures de nature variée. Certes, la tendance observée relative à la baisse de la durée du travail au travers de l'évolution du cadre juridique français apparaît également au niveau international. Mais la «norme sociale des quarante heures » avancée par les organisations internationales a-t-elle une traduction dans les pratiques ? Y a-t-il une mesure universelle de la durée du travail ?

L'Organisation Internationale du Travail (OIT) a bien tenté de définir un standard commun. En effet, dès 1962 la recommandation $n^{\circ} 116$ stipule le principe de la réduction de la durée normale du travail (BIT, 1984, p. 7) $:$ «La durée normale devrait être progressivement réduite, lorsque cela est approprié, en vue d'atteindre la norme sociale des quarante heures sans aucune diminution du salaire des travailleurs au moment où se produit cette réduction ». On peut identifier derrière cette recommandation, l'existence d'une mesure d'apparence universelle : la durée normale du travail. De quoi s'agit-il ? Le paragraphe 1.1 de la recommandation $\mathrm{n}^{\circ} 116$ définit la durée normale du travail, comme formant le nombre d'heures fixées par la législation, par accords collectifs, par sentences arbitrales ou en application de ceux-ci ou, à défaut, le nombre d'heures au-delà duquel tout travail effectué est rémunéré au taux des heures supplémentaires ou constitue une exception aux règles ou usages admis pour l'établissement ou les travaux considérés. En effet, cette définition s'écarte de la législation en intégrant une série d'indicateurs autres qui peuvent fixer un standard commun pour encadrer ce qu'on peut entendre par « durée normale $»$.

\footnotetext{
${ }^{4}$ Bureau International du Travail (1984). Le temps de travail. Genève.
} 
En revanche, le Bureau International du Travail admet que cette notion peut être peu significative dans la mesure où la durée effective du travail est variable au niveau national et international. C'est en effet la notion de travail effectif, préliminaire au calcul de la durée du travail, qui peut être interprétée de manières diverses, incluant ou non par exemple les périodes de repos ou de disponibilité (op cité, p. 31).

Les différentes mesures de la durée du travail peuvent se lire effectivement comme des principes d'identification face à l'usage de la «norme sociale». On peut constater que la durée normale du travail est en principe identique pour une même catégorie de personnel (ouvrier, employé). La durée affichée du travail segmente le personnel selon l'espace, à savoir selon l'unité de production (atelier, bureau). La durée effectivement offerte prend en compte les variations saisonnières comme les heures supplémentaires et le chômage partiel. De plus, puisqu'il s'agit d'une mesure collective, celle-ci n'inclut pas les variations individuelles dues aux absences ou aux heures supplémentaires individuelles. C'est la durée effective du travail qui prend en compte ces facteurs individuels en mesurant la durée réelle de présence sur les lieux de travail. Celle-ci n'intègre pourtant, ni les pauses, ni les temps d'inactivité (Doyelle, 1982).

Face à cette inflation d'indicateurs complémentaires voire contradictoires, nous présenterons brièvement ceux qui sont le plus utilisés en France à l'heure actuelle.

La durée hebdomadaire offerte du travail mesure en principe la durée collective affichée par l'entreprise, sans la prise en compte d'événements imprévus, mais elle intègre des heures supplémentaires collectives et le chômage partiel ${ }^{5}$. Elle est mesurée par l'enquête trimestrielle ACEMO effectuée sous la responsabilité du ministère du travail. Cette enquête exclut néanmoins les établissements de moins de 10 salariés et elle ne se rapporte qu'aux salariés non-postés et à temps complet. La durée annuelle effective du travail est censée d'intégrer tous les éléments individuels et collectifs non pris en compte par la durée hebdomadaire offerte. Cette mesure s'intéresse donc moins aux durées collectives qu'à l'effet que celle-ci produit sur les individus. La base de calcul se fait en partant de la durée hebdomadaire offerte (ACEMO). ${ }^{6}$ Celle-ci est multipliée par le nombre de semaines travaillées. Ensuite ce chiffre est corrigé en vue d'intégrer le travail à temps partiel

5 Sans la prise en compte des variations saisonnières on obtient la durée théorique du travail.

6 Il s'agit ici de l'indicateur utilisé dans l'enquête trimestrielle "Activité et les Conditions d'Emploi de la Main d'oeuvre » (ACEMO) sous la responsabilité du Ministère du Travail et de la Formation Professionnelle. On appelle cet indicateur aussi la durée hebdomadaire moyenne. 
(DARES, 1995) ${ }^{7}$. Ainsi L'INSEE (comptes nationaux) depuis 1970, mesure par l'enquête «emploi », chez les individus, la durée annuelle effective du travail en incluant l'absentéisme, les heures supplémentaires individuelles et les congés, ainsi que le travail à temps partiel ${ }^{8}$.

Enfin, la durée habituelle est, elle aussi, une mesure du temps passé par l'individu dans l'entreprise, sans toutefois prendre en compte les variations irrégulières comme les congés maladies et les heures supplémentaires occasionnels.

Les deux manières couramment utilisées pour présenter l'évolution la durée du travail concernent la durée hebdomadaire offerte (ACEMO), ou la durée annuelle effective du travail (INSEE). La multiplicité des mesures utilisées nous renvoie en effet à la difficulté de saisir l'impact des normes juridiques sur la durée du travail. Tantôt, la durée du travail est collective, tantôt, elle est individuelle. Les exclus de la mesure de la durée offerte comme par exemple les salariés à temps partiel, ceux qui travaillent en continu et ceux qui travaillent dans des entreprises de très petite taille, peuvent se retrouver dans la mesure des durées effectives. L'hétérogénéité des indicateurs de la durée du travail témoigne d'une activité intense et variée de la codification du cadre temporel.

\section{III.2. La durée hebdomadaire du travail entre 1891 et 1995 en France : 4 phases}

La question des durées pratiquées du travail a fait l'objet de divers travaux. Tout au long de cette partie, nous allons explorer cette littérature et les diverses données statistiques. D'une part, nous recourrons à des données produites par les organismes statistiques comme l'INSEE, la DARES (Ministère du Travail), ou des organismes internationaux comme l'OIT et l'office statistique de la Commission Européenne (EUROSTAT). D'autre part, nous nous référerons à des travaux utilisant ces données ou à des sources propres. Notre objectif consiste à retracer l'évolution des durées du travail observées, pour comprendre en quoi celles suivent ou non l'évolution des normes juridiques. Nous nous concentrons d'abord sur la question de la durée hebdomadaire, dont nous avons vu que, pour la France, elle a été au centre des codifications portant sur le temps de travail, après la durée journalière. Cette question sera traitée sous la forme d'une périodisation (phases), dont nous pensons qu'elle possède une cohérence interne.

\footnotetext{
${ }^{7}$ DARES (1995). Durée du travail et conjoncture, Premières Synthèses, 82. 
La baisse générale de la durée du travail est, certes une tendance lourde, mais fortement nuancée par les contextes réels : ces contextes vont faire apparaître, d'une part les fluctuations de la durée (en hausse ou en baisse) selon les périodes et d'autre part, la variabilité de ces durées selon les secteurs et selon les individus. En revanche, si nous limitons notre période d'observation des durées pratiquées, lors de la dernière décennie, nous constatons que la stagnation de la durée hebdomadaire du travail contredit, sur le court terme, les résultats de l'analyse historique des durées du travail sur un siècle (18901995). L'explication de cette stagnation devra être cherchée dans les manières de voir et de traiter le temps de travail, ce que nous ferons ultérieurement.

Enfin, pour saisir l'ampleur de la fin de la réduction de la durée hebdomadaire, nous élargissons notre perspective au niveau européen, afin de comprendre (1) la spécificité du cas de la France, en particulier du point de vue du rôle que joue la loi sur les durées effectives du travail, et (2) pour comprendre, si la tendance des durées pratiquées des autres pays industrialisés suit celle observée en France. La question européenne mérite par ailleurs notre attention, puisque l'évolution des durées du travail dans l'Union Européenne pourrait être un des indicateurs sociaux pour l'état de convergence des sociétés européennes.

Si nous analysons l'évolution de la durée hebdomadaire, au travers de l'indicateur de la durée hebdomadaire du travail (moyenne des durées offerte), nous constatons, en nous appuyant sur des sources diverses, que sur un siècle, la durée hebdomadaire du travail est passée de 65,19 heures en 1891 à 39 heures en 1995, soit une diminution de 40,2 heures. Nous pouvons présenter cette évolution en 4 phases distinctes : elle se caractérise par une baisse de 65,2 heures (1891) à 39 heures (1995) soit une baisse de 40,2\% sur un siècle ${ }^{9}$ :

1891-1936 : une forte réduction de la durée hebdomadaire offerte du travail (-25,2 heures)

1937-1966 : une période d'augmentation de la durée du travail (+5 heures)

1967-1983 : une période de réduction de la durée hebdomadaire du travail (- 6 heures)

1984-1995 : une période de stagnation de la durée hebdomadaire (=)

9 données: MSTEF-DARES (enquête ACEMO), bulletin mensuel des statistiques du travail, $\mathrm{n}^{\circ} 10-11$, 1995, INSEE, Marché du travail, séries longues, emploi-revenus, no 62-63, Paris, 1995. INSEE, annuaire rétrospectif de la France de 1948 à 1988, Paris, 1989. Découflé et Svendsen (1984), Naville (1972). 


\section{III.2.A. Une période de forte réduction de la durée du travail de 65,2 heures à 40 heures (- 25,2 heures) : 1891-1936}

Il fallait attendre l'enquête de l'office du travail (1891-1896) pour avoir une vision détaillée de la durée du travail dans les différentes branches industrielles. En effet, cette enquête permet pour la première fois, de calculer le nombre moyen de jours de marche de l'entreprise, le nombre de jours effectivement travaillés par an, la durée journalière moyenne et la durée effective du travail. L'office du travail va inaugurer en France la récolte systématique de données sur la durée du travail.

C'est ainsi que nous disposons aujourd'hui de données pour décrire l'évolution de la durée hebdomadaire du travail. En effet, selon les données rassemblées par Découflé et Svendson (1984) s'appuyant sur un éventail de sources différentes (ministère du travail, sources syndicales, patronales et parlementaires), la durée hebdomadaire du travail passe de 65,19 heures en 1891, tout secteur confondu, à 45,8 heures en 1931. En quarante ans, la durée hebdomadaire aurait ainsi diminué de 20 heures, ce qui représenterait aujourd'hui, pour donner une équivalence, un passage d'un temps plein à un travail à temps partiel.

Cette première période (1891-1937) contribuera en effet, de manière forte, à la réduction hebdomadaire de la durée du travail, mais l'évolution chronologique est caractérisée par des variations. La durée hebdomadaire, par exemple, passe en 1906 de 55,5 h à 58,4 h en 1910, pour redescendre à 55,5 h en 1913. La législation du temps de travail et l'évolution des négociations sur le temps de travail réduisent ensuite la durée à 40 heures en 1937. Les lois du début du XXe siècle instituent successivement la journée des 10 heures, le repos hebdomadaire obligatoire (1906), et la journée des 8 heures (1919). Un événement-clé, par rapport à la durée du travail, a été la grève générale de 1936, aboutissant à la loi sur les quarante heures et les 2 semaines de congés payés. Dans l'ensemble de l'industrie, la durée hebdomadaire diminuera de 4 heures en une année passant de 44 h (1936) à 40 h (fin 1937). Au cours de cette première période, la durée hebdomadaire du travail s'est alors réduite d'environ $39 \%$.

\section{III.2.B. Une période d'augmentation de la durée du travail de 40 heures à 45 heures} (+ 5 heures) : 1937-1966

La période suivante est caractérisée par une remise en question des pratiques temporelles en vigueur avant le mouvement de 1936. Les périodes de guerre apparaissent dans ce contexte défavorable à la réduction du temps de travail: Daladier autorisera pour les industries touchant la défense nationale, le passage de $40 \mathrm{~h}$ à $48 \mathrm{~h}$ (durée légale) par 
semaine en 1938, et pour les industries travaillant sous contrôle de la défense nationale le passage à la semaine des 60 h (6 x 10), dérogeant ainsi aux principes formulés en 1936. Après la libération et pendant la période de reconstruction, la législation sur les heures supplémentaires reste (depuis 1938) peu contraignante. La durée hebdomadaire moyenne s'établit à 45 h en 1955. La période de stagnation de la durée hebdomadaire va alors se prolonger jusqu'en 1967.

Sur l'ensemble de la période, on constate par voie de conséquence, un allongement de la durée hebdomadaire moyenne du travail. Celle-ci se voit d'une certaine manière compensée par l'augmentation des congés annuels à 4 semaines (1956, 1963). L'augmentation des congés et l'augmentation de la durée hebdomadaire moyenne du travail sont deux mouvements distincts, mais en les agrégeant, on observe une augmentation des durées du travail qui s'expliquerait par les efforts collectifs de rattrapage, et par les modifications des valeurs individuelles après la dernière guerre (Marchand \& Thelot, 1991).

\section{III.2.C. Une période de réduction de la durée hebdomadaire du travail de 45 heures à 39 heures : 1967-1983}

Ce n'est qu'avec le mouvement de mai-juin 1968, mais pour certaines branches, avant la signature des accords de Grenelle, qu'on renoue avec une réduction significative de la durée du travail (Bloch-London \& Marchand, 1990). Celle-ci va se prolonger jusqu'en 1984.

La première étape fait passer la durée du travail de 45 h (1967) à 40,6 h (1981). Cette évolution va de pair avec le rétrécissement continu de l'écart entre ouvriers et employés, qui passe de 1 h (44,7 h-43,7 h) en 1969 en faveur des employés, à moins de vingt minutes d'écart en 1982 (40,4 h pour les ouvriers, 40,1 h pour les employés). On assiste à une homogénéisation progressive des durées du travail entre les catégories d'ouvriers et d'employés. En revanche, les baisses globales des durées du travail ne commencent à devenir significatives qu'à partir de janvier $1968: 45 ; 5$ h pour les ouvriers contre 46,2 h en janvier 1967 ; et, c'est à ce moment aussi qu'a débuté un mouvement de conventions collectives par branche contribuant à la baisse des durées du travail. Le mouvement qui tend vers une homogénéisation des durées moyennes, se manifeste de telle sorte que les réductions sont d'autant plus importantes que la durée initiale du travail est élevée. Ceci est valable pour l'écart entre les différents secteurs et pour l'écart entre la catégorie ouvriers et employés. 
La deuxième étape commence avec une nouvelle réduction de la durée du travail au moment des lois Auroux qui réduisent la durée hebdomadaire légale à 39 heures, ce qui se traduit par une diminution des durées pratiquées de 40,3 heures à 39,2 heures entre 1982 et 1983 .

\section{III.2.D. La décennie après les lois Auroux : la stabilité de la durée hebdomadaire à}

\section{9 heures : 1984-1995}

Au cours de la mise en œuvre des ordonnances consécutives aux lois Auroux de 1982, la durée hebdomadaire légale est abaissée à $39 \mathrm{~h}$. La cinquième semaine de congés payés est généralisée et le contingent annuel des heures supplémentaires est fixé à $130 \mathrm{~h}$. La durée hebdomadaire moyenne du travail est fixée à $35 \mathrm{~h}$ pour les salariés postés continus. En revanche, il devient possible de déroger de manière conventionnelle à toute disposition concernant le temps de travail. Ces dérogations peuvent porter sur le repos dominical, sur les heures supplémentaires (modulation sur l'année), sur la modification des plages horaires d'interdiction du travail de nuit des femmes et la mise en place des horaires individualisés. Par ailleurs, les lois Auroux obligent à la négociation annuelle du temps de travail en entreprise. Malgré la réalisation de ces éléments, la durée hebdomadaire moyenne restera stable pendant une décennie jusqu'à aujourd'hui. Elle va s'établir à 39 heures en 1995.

La loi Aubry (1998) prévoit la réduction de la durée légale du travail à 35 heures. Les négociations de conventions collectives et d'accords d'entreprise en vue d'établir cette nouvelle norme sont en cours. Des incitations financières pour le maintien et la création d'emploi sous forme d'une réduction des charges sociales pour l'employeur pourrait avoir des effets sur les durées pratiquées. La règle juridique des 35 heures sera-t-elle de même effectivité sociale que celle sur les 39 heures en 1982? Peut-elle être le cadre d'une nouvelle diminution des durées pratiquées?

En résumé, nous assistons dans l'ensemble, premièrement, à une forte réduction de la durée du travail sur un siècle. Celle-ci est essentiellement imputable à la première période (-39\%). La norme des quarante heures, à laquelle on aboutit avec la loi de 1936, est mise en cause par la période suivante. Cette troisième période descend au niveau des durées de 1937 pour finalement remplacer la norme des 40 heures par les 39 heures. La dernière période confirme dans son ensemble la durabilité relative des nouveaux standards établis en 1982 . 
Soulignons deuxièmement l'homogénéisation des durées hebdomadaires entre les secteurs. Si en 1891 la différence entre le textile (68,82 heures par semaine) et la construction $(61,73 \text { heures })^{10}$ représentait encore 7 heures par semaine, celle-ci s'est réduite en 1995 (38,92 et 39,26 heures) à une demi-heure.

Enfin troisièmement, le rapport entre les durées du travail mesurées et les normes juridiques est variable selon les phases. Pendant la période précédant la loi sur les 40 heures, la durée légale (48 heures) dépasse les durées mesurées dans les entreprises (45,8 heures en 1931). Dans la période suivante, très rapidement les durées observées vont rattraper et dépasser la durée légale. Ce n'est que depuis 15 ans que la durée légale (39 heures) coïncide avec la durée hebdomadaire mesurée dans les entreprises. Cela ne signifie pas pour autant que les individus travaillent effectivement 39 heures, du fait notamment, des heures supplémentaires.

\section{III.3. L'Europe et la France: une évolution comparable des durées hebdomadaires du travail?}

\section{III.3.A. La codification du temps de travail à la lumière des durées pratiquées}

En un siècle, la durée hebdomadaire du travail en France a baissé de plus de $40 \%$. Cet énoncé peut inspirer des questionnements divers. Tout d'abord, c'est la question de la nature des faits observés permettant d'exposer une pratique sociale. Comment sommesnous passés d'un allongement des durées du travail à la baisse de la durée du travail ? L'interrogation porte non seulement sur la fiabilité des sources, mais plus encore sur le sens de la mesure elle-même. Pourquoi mesure-t-on la durée hebdomadaire du travail et non la quantité du travail effectuée mensuellement ? Que mesure-t-on : la présence sur le lieu de travail, la durée des opérations, le trajet domicile - lieu de travail, les repas et les pauses?

Ensuite, on peut poser la question du rapport social à la mesure. Donner une visibilité extérieure à un ensemble de situations extrêmement hétérogènes peut être considéré comme un coup de force. Il se pose la question de ce que cette norme recouvre en particulier à l'égard des divisions sociales qui disparaissent miraculeusement dans l'action de la standardisation. Le standard des 40 heures semble en effet posséder une force intrinsèque. Il est arithmétique : 5 fois 8 heures font quarante heures. Les 24 heures journalières se laissent combiner naturellement en trois équipes à 8 heures, Hébert (1992, p. 6). On peut

10 estimé selon Découflé et Svendson (1984) op. cité. 
constater que, pour le cas de la France, l'effectivité des 39 heures à partir de 1983 est toujours conditionnée par une division en équipes basée sur le calcul de la semaine de 7 jours, 24 heures et 8 heures, bref selon un référentiel établi en 1936. La simplicité du modèle domine même dans les pays et les secteurs qui affichent des durées inférieures. La durée normale (conventionnelle) de la semaine de travail dans la métallurgie allemande est de 35 heures : en vigueur depuis 1995 cette durée ne change pas toujours la durée des postes ou le nombre de jours travaillés par rapport au modèle des 40 heures. En revanche, il semble que même les espaces professionnels très codifiés par un ensemble de règles légales et conventionnelles autorisant des dérogations et des variations, comme le montre par exemple, l'analyse des lois et des conventions passées au Québec (op cité). Nous y trouvons une définition variable selon le secteur, de la semaine normale de 5 x 7 ou 8 heures ( 35 heures ou 40 heures), et des seuils de déclenchement variables pour les primes des heures supplémentaires. Le travail en semaine comprimé à 4 jours peut rallonger les temps de présence jusqu'à 10, voire 12 heures (sans transport) ; cette pratique renvoie au paradoxe d'une durée journalière monopolisée par le travail professionnel et d'une semaine de travail raccourcie avec l'extension du week-end à trois jours (op cité). Les baisses observées de la durée hebdomadaire sont donc à relativiser par d'autres codifications plus complexes (formes d'organisation) des durées pratiquées sur la semaine.

Ces réflexions sont confirmées par l'évolution de la durée hebdomadaire que nous venons d'exposer. En France, les durées pratiquées du travail ont baissé sensiblement au cours d'un siècle. Par contre, le mouvement à la baisse n'est ni uniforme, ni mécanique. Nous sommes au contraire parvenus à la conclusion que des événements singuliers, comme des mouvements sociaux ou comme les périodes de guerres ont des effets à la fois sur l'établissement de la réglementation du temps de travail, mais aussi sur l'évolution des durées pratiquées. Le rapport entre la norme juridique et les durées effectives ne peut pas faire l'objet d'une relation mécanique, dans laquelle l'un serait la conséquence inévitable de l'autre.

\section{III.3.B. France et Union Européenne}

L'autre question concerne la spécificité du cas français. La réglementation et les pratiques de la durée du travail peuvent-elles être décrites comme interdépendantes (synchronisées) ou décalées?

Nous avons vu que les lois sur les quarante heures en 1936, comme celle sur les 39 heures en 1982 ont été suivies d'une baisse des durées pratiquées. Cette baisse a été provisoire 
dans le premier cas, alors qu'elle semble être durable dans le second. Peut-on alors constater une normalisation juridique des durées pratiquées dans les autres pays européens à l'image de l'évolution en France?

Les durées habituelles du travail observées dans l'Union Européenne entre 1983 et 1995 nous permettent de montrer que, même dans des pays industrialisés proches, l'évolution est disparate. Quel est le rapport entre les normes légales et les durées pratiquées dans l'Union Européenne ? Sur fond d'une hétérogénéité des durées nationales en 1983, certains pays semblent sur la voie de la réduction de la durée hebdomadaire, alors que dans d'autres pays, celle-ci stagne ou augmente.

Selon les données provenant de l'enquête sur les forces de travail (EUROSTAT, 1995 et 1996) ${ }^{11}$ sur la base de la durée hebdomadaire habituelle du travail (excluant le temps partiel et les variations irrégulières de la durée du travail), 12 pays de l'Union Européenne se laissent néanmoins répartir selon certaines caractéristiques. D'abord, nous pouvons constater (Fig.1), que les durées du travail restent relativement stables sur les treize dernières années (1983-1995) dans l'Europe des 12. La durée habituelle du travail baisse d'environ 15 minutes par semaine en passant de 40,6 heures à 40, 3 heures. La moyenne se fait ici sur une longueur d'intervalle de durées nationales plus importantes en 1995 qu'en 1983. En 1995, 5,5 heures séparent la durée habituelle, maximale de la durée minimale. Les durées se distribuent entre 38,4 heures (Italie, Belgique) et 43,9 heures (Royaume-Uni) par semaine.

${ }^{11}$ EUROSTAT, Enquête sur les forces de travail - résultats de 1995, Luxembourg, Office Statistique des Communautés Européennes, p. 168, tableau 073, 1996.

EUROSTAT, Organisation et durée du travail 1983-1992, Luxembourg, Office Statistique des Communautés Européennes, p. 28, tableau I/4. 1995. 


\begin{tabular}{|l|l|l|l|l|l|}
\hline & $\begin{array}{l}\text { Durée habit. } \\
\text { Durée habit. }\end{array}$ & $\begin{array}{l}\text { Différence } \\
1983\end{array}$ & $1995-1983$ & $\begin{array}{l}\text { Durée légale } \\
1993\end{array}$ & $\begin{array}{l}\text { Ecart entre } \\
\text { durée légale } \\
\text { et habituelle } \\
1995\end{array}$ \\
\hline Royaume-Uni & 43,9 & 42,3 & $+1,6$ & - & - \\
\hline France & 39,9 & 39,7 & $+0,2$ & 39 & $-0,9$ \\
\hline Irlande & 40,2 & 40,2 & 0 & 48 & $+7,8$ \\
\hline Belgique & 38,4 & 38,6 & $-0,2$ & 40 & $+1,6$ \\
\hline Espagne & 40,7 & 41,1 & $-0,4$ & 40 & $-0,7$ \\
\hline Luxembourg & 39,5 & 40 & $-0,5$ & 40 & $+0,5$ \\
\hline Grèce & 40,3 & 41 & $-0,7$ & 48 & $+7,3$ \\
\hline Italie & 38,4 & 39,2 & $-0,8$ & 48 & $+9,6$ \\
\hline Portugal & 41,2 & 42,3 & $-1,1$ & 48 & $+6,8$ \\
\hline Allemagne & 39,7 & 40,8 & $-1,1$ & 48 & $+8,3$ \\
\hline Pays-Bas & 39,5 & 41 & $-1,5$ & 48 & $+8,5$ \\
\hline Danemark & 38,9 & 40,5 & $-1,6$ & - & - \\
\hline Ensemble & 40,3 & 40,6 & $-0,3$ & 48 & $+7,3$ \\
\hline Sources: & & & & & \\
\hline
\end{tabular}

Sources : Eurostat $(1993,1995,1996)^{12}$

Figure 1 : Evolution de la durée habituelle et légale de la semaine de travail (en heures) dans l'Europe des 12

L'évolution de la durée habituelle entre 1983 et 1995 nous montre un premier groupe de pays qui maintiennent à peu près stable leur durée habituelle, comme l'Irlande, la France ( $+0,2$ heures) et la Belgique (- 0,2 heures). Un second groupe de pays connaît des baisses modérées comme l'Espagne, le Luxembourg, la Grèce et l'Italie, où les durées du travail ont connu un recul de 0,4 à 0,8 heures. Un troisième ensemble concerne des pays dont la baisse est plus forte (de 1,1 à 1,6 heures) comme le Portugal, l'Allemagne, les Pays-Bas et le Danemark. A l'opposé le quatrième groupe, composé par le Royaume-Uni augmente de manière significative sa durée hebdomadaire déjà élevée de 1,7 heures.

12 EUROSTAT, Organisation et durée du travail 1983-1992, Luxembourg, Office Statistique des Communautés Européennes, p. 28, tableau I/4. 1995. EUROSTAT, Enquête sur les forces de travail - résultats de 1995, Luxembourg, Office Statistique des Communautés Européennes, p. 168, tableau 073, 1996. Commission Européenne, Bulletin sur les femmes et l'Emploi dans l'UE, no 7, Bruxelles, 1995. Les chiffres pour l'Espagne, le Portugal et l'Europe des 12 en 1983 correspondent aux valeurs relevées par Eurostat en 1987. 
Les pays (Fig. 1) sont classés selon l'ampleur de leur baisse de la durée du travail sur la période 1983-1995 (colonne 4). La comparaison de l'écart entre la norme légale et la durée habituelle en 1995 (colonne 5) montre (1) que la durée légale n'existe pas partout, (2) quand elle existe, elle peut être supérieure ou inférieure à la durée habituelle. (3) L'écart aux durées légales en 1995 varie de +10 heures environ pour l'Italie à $-0,9$ heures pour la France. Ceci indique une généralisation difficile de l'influence des normes juridiques sur les durées pratiquées dans les pays européens.

En intégrant d'autres variables, comme le taux d'activité, l'évolution peut être catégorisée de manière différente. L’interprétation avancée par Boisard (1995) des durées habituelles pour l'Europe des 12 met en valeur l'opposition de deux pôles de répartition de la durée du travail. Un premier pôle regroupe les pays à faible durée hebdomadaire, mais à fort taux d'activité comme le Danemark, les Pays-Bas et l'Allemagne. Le pôle opposé intègre des pays à durée hebdomadaire relativement élevée, combiné avec un faible taux d'activité : France, Irlande et Luxembourg.

En tous cas, on n'observe ni une baisse générale et forte, ni une convergence des durées européennes, ni un rattrapage des durées courtes par les pays qui ont des durées habituelles plus longues. En plus, nous pouvons constater que les durées légales n'exercent pas une influence homogène sur les durées habituelles. Certains pays dont la durée légale est fixée à 48 heures, pratiquent des durées habituelles beaucoup moins importantes (Grèce, Italie, Portugal, Allemagne, Pays-Bas). D'autres pays ont une durée habituelle proche de la durée légale (France, Belgique, Espagne, Luxembourg). Enfin deux pays européens ne connaissent pas le principe de la durée hebdomadaire légale : le Danemark qui la durée habituelle la plus basse et le Royaume-Uni qui a la durée la plus élevée dans l'Europe des 12. En revanche, une directive européenne qui limite la durée légale hebdomadaire du travail à 48 heures devrait s'appliquer aussi au Danemark et au Royaume-Uni.

Nous pouvons déjà conclure de cette description, premièrement, que la durée légale ne structure pas les durées pratiquées de la même manière dans les pays européens. Aucune régularité européenne se dessine. Deuxièmement, en ce qui concerne la baisse de la durée du travail et le niveau des normes légales, on pourrait aussi énoncer de manière polémique que plus la durée légale est élevée (48 heures), plus les durées habituelles ont baissé, entre 1983-1995 (colonne 3, 4). En effet, tous les pays ayant les durées légales plus élevées (sauf le Portugal), possèdent des durées habituelles relativement courtes (en-dessous de la moyenne) ; de plus, tous ces pays sont sur la voie de la réduction de la durée hebdomadaire 
du travail. Il ne s'agit pas de démontrer l'inefficacité de la réglementation dans le domaine du temps de travail. En revanche, ces données nous mettent en garde contre une interprétation univoque et à-historique des règles juridiques coupées de leur milieu d'élaboration et d'application.

La comparaison des durées habituelles avec les durées hebdomadaires légales met en évidence la variabilité de l'écart entre les durées habituellement pratiquées et la norme juridique. Ainsi, nous constatons des durées habituelles hebdomadaires très inférieures aux normes juridiques aux Pays-Bas, en Allemagne, en Grèce, en Italie et en Irlande. Cependant, nous pouvons retrouver également des pays dans lesquels la durée habituelle du travail dépasse les plafonds légaux établis, comme c'est le cas en Espagne et en France.

En résumé, le contexte européen des durées légales et habituelles montre un ordre complexe des durées hebdomadaires. Observons que la durée habituelle du travail, sur l'ensemble des pays européens et sur la période récente (1983-1995) diminue peu (de 40,6 à 40,3 heures), ce qui confirmerait la tendance observée à la stagnation en France. Par ailleurs, nous pouvons constater qu'au niveau européen, les durées légales basses du travail n'encouragent pas la réduction des durées habituelles. Pour cet aspect, l'histoire des durées du travail en France nous aurait plutôt fait penser à une structuration forte de la durée du travail en Europe par les normes légales. La baisse hétérogène et peu sensible de la durée du travail au niveau des 12 pays pourrait plutôt décrire la désynchronisation des durées européennes, depuis le début des années 1980.

\section{CONCLUSION}

\section{IV.1. Une conception du temps centrée sur le temps industriel}

La première conclusion est que le temps de travail est une invention récente qui correspond à une manière de voir les activités humaines : cette conception met au centre les activités professionnelles; le temps des affaires devient une affaire de temps et l'économie de temps devient, au siècle dernier, le principe d'organisation de ces activités. Le temps devient abstrait, indifférencié, monochrone : c'est le temps de la besogne et du gain. La conception du temps qui s'installe au XIXe siècle est celle du temps industriel : avec d'un côté, le temps qui encadre le rapport salarial et qui est caractérisé par le temps de la subordination du salarié à l'égard de son employeur; et de l'autre côté, le temps de la rationalisation basé sur l'économie de temps. Pour autant, il ne faudrait pas conclure que 
ce temps dominant est unique: il cohabite avec d'autres temporalités qu'il tente d'englober.

\section{IV.2. L'importance de la réglementation du temps de travail}

La deuxième conclusion concerne la lente montée de la réglementation. On aurait pu penser que le temps de travail est une simple affaire de décret, le résultat mécanique de l'intervention de la puissance publique. Certes, le temps de travail est le premier domaine d'intervention de l'État, qui devient la puissance publique normative, le fondateur et le garant de l'ordre public social. Mais le débat autour du rapport de Villermé sur la condition des enfants au travail, montre que dans le domaine du temps de travail, on ne gouverne pas par décret. La formulation d'une loi et son adoption par les deux chambres suppose des débats d'une actualité étonnante. En effet, les débats animés par les opposants à la réglementation, tournent dès 1835 sur la nécessité d'une loi, jugée par eux, inapplicable, peu pertinente pour régler de manière égale les problèmes qui se posent tant au Nord que dans le Midi. Ces opposants avancent que la loi se substitue à l'autorité de l'employeur ou pire à celle des parents. Rien n'arrêtera la montée des règlements : ils vont freiner l'intensité du travail qui s'est développée au siècle dernier et se traduire par des mesures de protection de la santé des individus qui travaillent. Ces dispositifs vont avoir quatre caractéristiques essentielles: (a) l'existence de normes temporelles touchant la durée journalière, puis hebdomadaire, les repos et les congés ; (b) l'intervention de l'État comme puissance normative, (c) la règle commune qui crée le collectif de ses destinataires et enfin (d) les moyens de la loi, c'est-à-dire les inspecteurs du travail qui doivent faire respecter la loi.

\section{IV.3. La durée comme figure emblématique du temps de travail}

Troisièmement, il reste à savoir ce que les individus font de la loi et comment elle se traduit dans les faits. L'examen des pratiques montre qu'il n'y a pas de lien automatique entre la loi et les pratiques. Cependant, une norme sociale est née : celle de la durée du travail hebdomadaire, qui devient la figure emblématique de la norme temporelle ; elle occupe désormais une place centrale dans l'ordre temporel, pour plusieurs raisons. D'abord, la durée hebdomadaire constitue le référentiel le mieux partagé pour parler du temps de travail : elle est fixée par la loi, objet de luttes sociales. Ensuite, cette durée sert de mesure, de base de calcul (notamment des salaires) et de comparaisons pour le temps de travail, même si comme nous le verrons, cette base peut être établie selon plusieurs critères. Enfin, la durée du travail, qui s'est établie autour des 40 heures, constitue une 
référence symbolique, qui trône au milieu de toutes les autres références : elle constitue le principe d'articulation du temps des activités professionnelles et du temps des activités non professionnelles.

\section{IV.4. Durées pratiquées: baisse sur un siècle, mais quatre phases contradictoires}

Quatrièmement, l'évolution de la durée du travail hebdomadaire nous indique sur un siècle une baisse importante de celle-ci en France, clôturée par une période de stagnation. Cependant, 4 phases se distinguent. La dernière période d'observation (1983-1995) nous suggère la fin provisoire de la réduction hebdomadaire et sur l'ensemble des 3 dernières phases (à partir de 1937) le niveau de la durée hebdomadaire évolue très lentement. En revanche, les points de ruptures de 1937, 1967 et 1983 indiquent une variabilité des phases qui semblent posséder une cohérence interne. Au regard de ces résultats, nous considérons que nous nous trouvons toujours dans une période de stagnation de la durée hebdomadaire qui s'est établie, depuis 1983, à 39 heures hebdomadaire. Ce processus est accompagné d'une harmonisation des durées du travail, d'une part entre les employés et les salariés et d'autre part, entre les différents secteurs d'activités. La convergence de la durée légale avec la durée hebdomadaire observée n'est qu'un phénomène relativement récent. Dans l'ensemble, sur le siècle qui vient de s'écouler, l'évolution des normes juridiques présente des écarts importants aux durées hebdomadaires pratiquées. De plus la situation européenne est caractérisée par des évolutions divergentes selon les pays.

Il nous reste à comprendre comment cette structure temporelle, caractérisée par ses quatre composantes (les normes temporelles, l'intervention de l'État, la règle commune et les moyens de la loi), va se comporter face aux transformations récentes, aux manières de voir le temps de travail, mais aussi face aux dispositifs juridiques et enfin aux pratiques. C'est l'objet du chapitre suivant, consacré au « temps éclaté ». 



\section{CHAPITRE II}

\section{L'ECLATEMENT DU TEMPS DE TRAVAIL}




\section{Introduction}

L'ordre temporel dominant qui a émergé au XIXe siècle, est celui qui encadre et organise les activités professionnelles; le temps de travail est bien le temps dominant qui résulte de la combinaison entre des pratiques, des représentations et un ensemble de règlements. Ce « temps légal » était jusqu'à une période récente une structure inébranlable. Pourtant, dans les années 1980 vont émerger des pratiques, des représentations et des dispositifs réglementaires différents. Peut-on parler d'un ordre temporel différent?

La loi Aubry sur les 35 heures, l'étape la plus récente de la réglementation, participe-t-elle à cette construction d'un ordre temporel différent ?

S'il y a bien une perte d'efficacité de certains dispositifs réglementaires et des modifications dans les formes d'intervention de l'État, il ne faudrait pas conclure trop vite à une déréglementation, voire à la disparition du temps de travail comme temps dominant. Pourtant, les faits sont là : la question de la durée du travail change de nature, puisqu'elle ne se pose plus en termes de réduction pour partager les gains de productivité, mais bien en termes de régulation du marché du travail comme l'attestent les travaux de Freyssenet (1997), rassemblés dans un ouvrage au titre évocateur : «le temps de travail en miettes ». La réduction de la durée du travail n'est plus qu'un moyen pour agir sur le maintien, voire la création d'emplois; la réduction de la durée du travail devient une contrepartie ou une compensation de l'aménagement du temps de travail. On pourrait penser qu'il ne s'agit là que d'une évolution banalisée par les exigences de compétitivité et résultant de la mondialisation. Peut-on faire comme si l'on pouvait appréhender les transformations du temps de travail selon un continuum ? Peut-on simplement considérer qu'il s'agit d'un ajout de paramètres qui sont désormais traités en même temps ? Peut-on interpréter ces modifications comme la solution aux problèmes de l'emploi ? Peut-on continuer à laisser croire qu'il s'agit d'une réponse à la prétendue rigidité des cadres réglementaires ?

L'objet de ce chapitre est de montrer que les transformations du temps de travail ne sont pas la solution aux questions d'emploi, mais d'abord un problème qu'il s'agit d'expliquer ; il ne s'agit pas d'une réponse aux rigidités du cadre réglementaire, mais d'une question relative à une autre façon de réglementer. Autrement dit, il faut bien mesurer la nature et l'ampleur des changements en cours au regard de la structure temporelle antérieure. 
Les règlements, des pratiques et des représentations se conjuguent pour définir autrement le temps de travail : le temps de travail devient un temps éclaté. ${ }^{13}$ Par " temps éclaté », on entend, premièrement, que le temps de travail n'est plus traité pour lui-même : il est fragmenté en une multitude de composantes (temps partiel, annualisation, repos compensateurs, travail du samedi, etc.) qui donne lieu à des règles différentes, notamment parce qu'elles remettent en cause les articulations avec les autres temporalités sociales; la variation de la durée hebdomadaire du travail et la remise en cause des deux jours consécutifs en fin de semaine en témoignent de manière significative. Le sens de la norme temporelle change. Par " temps éclaté », on entend, deuxièmement, le fait que les règles temporelles qui atomisent la structure temporelle sont tournées plutôt vers l'accroissement de la flexibilité de la production et vers la régulation du marché du travail que vers l'amélioration de la vie quotidienne des salariés. Le temps de travail n'est plus traité pour lui-même et les actions menées dans ce domaine ont une finalité externe au temps : les termes du compromis social (productivité-réduction de la durée du travail) changent au profit d'une nouvelle combinaison entre l'emploi, la flexibilité et le temps. La construction des normes se fait de manière différente : l'État n'a plus le monopole de la création normative puisque le temps de travail entre désormais dans le champ de la négociation collective; la communauté des destinataires de ces règles communes se dissout en une multitude de collectifs aux règles de plus en plus individualisées.

Parler de «temps éclaté » comme fragmentation, comme remise en cause du compromis social et du mode de régulation ne signifie pas que l'ordre temporel des activités professionnelles se défait. L'ordre temporel ne disparaît pas, mais sa nature, son mode d'élaboration et sa légitimité changent. Il s'agit donc pour nous de tenter de saisir cette fragmentation et de comprendre comment des acteurs se regroupent ou s'opposent pour construire une structure temporelle ou pour entrer en conflit. La question fondamentale à laquelle ce chapitre essaie de répondre est la suivante : l'éclatement du temps de travail et l'atomisation de la structure temporelle sont-ils le moment de l'émergence d'autres configurations temporelles? Quelle est la nature de l'espace de négociation qui s'ouvre lorsque le temps de travail devient un objet qui entre dans le champ de la négociation collective?

13 C'est l'expression que nous avions utilisée lors de notre communication aux 6e Séminaire International sur le Temps de Travail (SITT) à Blankenberge en Belgique. Voir :Thoemmes, J.\& de Terssac, G.(1997b). 
Pour répondre à ces questions nous avons mené trois types d'investigations :

- une analyse de l'évolution des dispositifs réglementaires depuis 1980 pour indiquer de quelle manière le temps de travail entre en négociation (première partie),

- une analyse des « accords » d'entreprises portant sur le temps de travail en Midi-Pyrénées de 1984 à 1995 : au total 768 accords ont été traités (deuxième partie),

- enfin, une analyse de la mise en œuvre de la loi de juillet 1994 concernant le « compte épargne temps»: dix conventions collectives de branches ont été analysées (troisième partie).

L'objectif est aussi de mieux comprendre par ces données sur la négociation antérieure à la loi Aubry, sur quelle réalité sociale la loi intervient. Notre thèse est que l'analyse de la négociation du temps de travail des décennies 1980 et 1990 montre une rupture fondamentale des manières de faire du temps de travail. Cette rupture décrit un alors processus qui aide à comprendre les enjeux disputés actuellement autour de la loi sur les 35 heures.

\section{L'OUVERTE DE L'ESPACE DE NEGOCIATION : LE CONTEXTE JURIDIQUE}

Le contexte juridique du temps de travail a subi de profondes transformations que nous ne pouvons aborder exhaustivement. Il nous semble cependant que l'on ne peut pas aborder les figures temporelles qui vont se construire dans la négociation, sans donner quelques indications sur son contexte juridique : d'abord parce que les dispositifs réglementaires sont liés aux pratiques qu'elles visent parfois à institutionnaliser; ensuite parce que la négociation ne se déroule pas dans un vide social, mais dans un contexte déjà structuré, même si le résultat de la négociation n'est pas déterminé a priori.

Des contraintes pèsent sur la négociation du temps de travail, elles sont liées à un triple changement concernant (a) la conception émergeante du temps de travail, (b) le mode de régulation du temps de travail, enfin (c) la nature des règles temporelles.

\section{l.1. Un changement de la conception du temps de travail}

Si l'on veut bien mesurer la nature et la portée des modifications en cours, il faut se référer aux conceptions du temps qui changent : le temps de travail n'est plus synonyme de norme 
unique, applicable à tous et centrée sur la protection des personnes qui travaillent; le temps devient un moyen d'obtenir autre chose, d'améliorer la flexibilité de la production et de préserver les emplois.

\section{I.1.A. De la conception hygiéniste à la conception gestionnaire}

Le temps de travail est une construction sociale dont les dimensions et les fonctions sont multiples. Il est tout à la fois un élément de mesure du travail abstrait, un objet d'échange et un élément d'organisation du travail. Comme nous venons de le voir au chapitre précédent, il est aussi un élément de limitation et de protection de l'activité humaine concrète de travail, opérant le partage entre le temps de travail et le temps libre, partage qui définit les conditions de la vie quotidienne et structure les temps sociaux (Mercure, 1995 ; Naville, 1969). Jusqu'au milieu des années soixante-dix, la réduction du temps de travail et son aménagement étaient traités au chapitre de l'amélioration des conditions de travail ${ }^{14}$. Dans le cadre d'une réduction tendancielle de la durée du travail, celle-ci était un gage de progrès social fondé sur le partage des gains de productivité. Depuis 1982, ces enjeux se sont déplacés autour de la réduction de la durée du travail et de la flexibilité sous l'égide de l'emploi : il nous semble dès lors que l'entrée en négociation du temps de travail entraîne un changement de la manière de voir le temps de travail qui a tendance à devenir un moyen pour autre chose, une variable d'ajustement entre les acteurs pour maintenir l'emploi, un outil de marchandage entre employeurs et salariés pour faciliter la flexibilité de la production.

\section{I.1.B. Du corps à l'emploi}

Le thème de la réduction du temps de travail en France est au centre du débat social actuel ; ce thème a été peu abordé depuis 1936 en France et il aura fallu attendre 45 ans pour qu'il soit remis à l'ordre du jour et pour que ce débat se traduise par des mesures concrètes. En effet, premièrement, à partir de 1981 diverses mesures vont se combiner pour tenter d'apporter une contribution à la réduction du temps de travail ; néanmoins, ces mesures ont en commun de ne plus traiter de la durée du travail en soi, mais d'insérer cette durée dans une réflexion plus vaste touchant la relation entre le temps de travail, l'emploi et la performance de l'entreprise. On assiste depuis 15 ans à des transformations de l'organisation temporelle associées à une remise en question des conceptions traditionnelles du temps de travail. Deuxièmement, la loi modifie les conditions de négociation de l'aménagement du temps de travail. Elle privilégie la négociation

14 Loi du 27 décembre 1973 sur les horaires variables - Loi du 6 juillet 1996 sur le repos compensateur - Accord interprofessionnel de 1975 sur l'amélioration des conditions de travail. 
d'entreprise sur le travail en continu pour des raisons économiques et surtout le temps partiel (heures complémentaires, délai de prévenance); elle permet l'adoption du temps partiel annualisé par simple contrat de travail, en abrogeant les dispositions sur le travail intermittent qui nécessitait un accord de branche étendu. Elle autorise enfin l'adoption du repos compensateur de remplacement, en l'absence de délégué syndical, après consultation des représentants élus, sauf opposition de leur part.

Sans détailler les mesures récentes qui ont été prises, nous nous proposons de les retracer succinctement afin de dégager les principaux éléments qui viennent remettre en question l'univers des normes temporelles de travail (Morin, de Terssac, Thoemmes, 1998). Il nous semble en effet que cette remise en question porte, d'une part, sur la manière de produire des règles temporelles, c'est-à-dire sur le processus de régulation lui-même et, d'autre part, sur la nature des règles temporelles qui s'écartent de l'univers normatif qui a fonctionné jusqu'en 1980.

\subsection{Un changement du mode de régulation : du décret à la négociation}

L'évolution des textes indique que l'on s'oriente vers la fin des normes générales, égalitaires et protectrices, fixées de manière réglementaire.

\section{I.2.A. Le tournant de 1981}

La première transformation concerne le mode de régulation du temps de travail. On assiste, dans les années 80 , au passage d'un mode tutélaire de réglementation du temps de travail à un mode négocié ; la régulation centrale opérée par l'État devient locale puisque le temps de travail fera désormais l'objet de négociations et d'accords variables d'une branche à l'autre et d'une entreprise à l'autre (Morin et al., 1998).

L'accord interprofessionnel du 17 juillet 1981 ouvre la voie à ce changement du mode de régulation : il accepte le principe d'une réduction de la durée du travail (réduction de la durée légale, allongement des congés payés, etc.) contre un assouplissement des règles d'aménagement du temps de travail (modulation, équipes de fin de semaine, etc.). Le temps de travail est désormais traité comme une variable d'ajustement entre la logique sociale tournée vers la réduction de la durée du travail et la logique économique tournée vers l'amélioration de la compétitivité des entreprises. L'irruption légale de la négociation dans le domaine du temps de travail où elle ne figurait pas, résulte, d'une part, de l'obligation annuelle de négocier sur la durée et sur l'aménagement du temps de travail et, d'autre part, de l'introduction de possibilités de dérogations par la voie de la négociation conduisant aux accords de branche ou d'entreprise. 


\section{I.2.B. L'espace de négociation : durée, flexibilité, emploi}

Les différentes lois qui vont être promulguées au cours des 12 dernières années (19841996) ont en commun moins d'imposer des normes temporelles que d'ouvrir des espaces de négociations par le jeu d'un transfert de compétences de la loi vers les parties prenantes du contrat de travail (employeurs et salariés) : la flexibilité temporelle devient la contrepartie de toute réduction du temps de travail ; de plus, la durée conventionnelle est fixée par les entreprises qui se voient dotées de marges de manœuvre accrues et d'une plus grande souplesse pour construire des formes d'aménagement variées (Morin et al., 1998) L'ordonnance du 16 janvier 1982 qui a suivi l'accord interprofessionnel a consacré cette mutation. Elle opère le passage de $40 \mathrm{~h}$ à $39 \mathrm{~h}$, et à $35 \mathrm{~h}$ pour le travail continu : la durée légale du travail est réduite et la cinquième semaine de congé est accordée; mais toute réduction s'entend désormais en contrepartie d'un aménagement du temps de travail qui est en fait une remise en cause du cadre normatif. L'autre volet de l'ordonnance concerne la possibilité de déroger conventionnellement aux décrets pour assouplir le cadre normatif en contrepartie de la réduction de la durée : (a) elle introduit la possibilité de faire varier la durée hebdomadaire, à condition qu'en moyenne sur l'année celle-ci soit égale à 39 heures (modulation I), (b) elle permet de nouvelles formes d'aménagement du temps de travail qui dérogent à la règle de l'horaire collectif et du repos dominical, comme la mise en place d'équipes de fin de semaine et le travail par roulement désormais autorisés, et plus généralement (c) elle remet en cause la répartition de la durée du travail dans le cadre de la semaine. En donnant un rôle nouveau à la négociation de branche et d'entreprise, elle a conduit à lier la question de la réduction de la durée du travail à celle de son aménagement pour assurer la compétitivité des entreprises. La problématique de l'accord donnantdonnant qui s'affirme plus complètement à partir de 1984, deviendra désormais centrale.

Dès 1984 , le protocole d'accord du 16 décembre relie les questions de temps de travail à l'emploi.

La loi quinquennale du 20 décembre 1993 poursuit à cet égard le mouvement engagé depuis l'ordonnance du 16 novembre 1982 visant à flexibiliser le temps de travail, plus précisément à favoriser l'adoption négociée «d'arrangements temporels » diversifiés. Ces dispositifs nouveaux concernent aussi bien l'aménagement collectif que l'aménagement individuel du temps; ils visent (a) des possibilités de réduction de la durée du travail en contrepartie d'une flexibilité du temps de travail, (b) l'annualisation plus complète du temps de travail collectif (modulation de type III) et surtout individuel (temps partiel 
annualisé), et (c) des aménagements aux règles relatives à la répartition de la durée du travail sur la semaine. De plus, la loi quinquennale tente de relancer le mouvement de réduction négociée de la durée du travail dans une perspective d'emploi, mais dans ce but, elle le lie à l'extension de l'annualisation donnant aux entreprises plus de souplesse d'organisation et de fonctionnement.

En 1996, la loi de Robien revient plus fortement sur la finalité « emploi » de la réduction de la durée du travail. Celle-ci sera subventionnée par l'Etat dans le respect des engagements légaux que l'entreprise contracte sur l'emploi. La loi Aubry de 1998 sur les 35 heures généralise cette possibilité d'accord avec une nouvelle norme de la durée du travail à l'horizon ${ }^{15}$.

\section{I.3. Un changement de la nature des règles temporelles}

La deuxième transformation concerne la nature des règles temporelles. Le temps de travail a longtemps été en France du domaine de l'intervention de l'État : la législation de 1936 était fondée sur la définition d'une durée légale du travail, considérée dans le cadre de la semaine comme une norme égalitaire fixée de façon réglementaire. Depuis les années 1980 , le processus de remise en question des règles en vigueur s'accentue. Il porte (a) sur l'extension de l'annualisation du temps de travail, c'est-à-dire sur l'élargissement de l'horizon de calcul qui passe de la semaine à l'année avec une variation de la durée hebdomadaire effective, (b) sur l'augmentation des possibilités de délocalisation des périodes de travail, c'est-à-dire sur l'extension du travail de nuit notamment pour les femmes ou du travail du samedi, voire du week-end, (c) sur la montée en puissance du travail à temps partiel imposé plus que choisi et enfin (d) sur une réduction de la durée du travail sous réserve de contreparties dans l'adoption de mesures de celles que nous venons de présenter. La loi du 28 février 1986, dite loi sur la flexibilité, est essentiellement une loi inspirée du rapport Taddei qui ouvre des possibilités nouvelles d'aménagement des horaires en contrepartie d'une réduction du temps de travail. Ces possibilités étaient essentiellement relatives à l'annualisation des horaires et aux heures supplémentaires. Elle a introduit en effet un nouveau système de modulation (II), c'est-à-dire l'annualisation des horaires qui permet de répartir la durée du travail sur l'année, sans paiement de la majoration des heures effectuées dans les limites de variation de l'horaire hebdomadaire.

La loi du 19 juin 1987 définit comme contrepartie obligatoire d'une modulation la réduction de la durée du travail.

\footnotetext{
${ }^{15}$ Pour un aperçu plus détaillé des politiques d'emploi et de la réduction du temps de travail en France depuis la fin des années 1970 voir Freyssenet (1997).
} 
Ensuite, la loi quinquennale du 20 décembre 1993 confirme l'échange possible entre annualisation et réduction de la durée du travail (art. 38). En cas d'annualisation et dans le respect de la réduction de la durée du travail fixée sur l'année, les durées journalières peuvent atteindre de 10 à 12 heures (durées maximales) et les durée hebdomadaires de 46 à 48 heures (durées maximales ) sans que ces heures soient majorées. La loi incite avec une réduction des charges sociales à la réduction de la durée du travail afin de créer des emplois. A condition néanmoins que celle-ci s'accompagne d'une réduction des salaires et d'un accord sur l'annualisation du temps de travail, (art. 39).

La loi Aubry (1998) enfin n'est pas incompatible avec l'annualisation du temps de travail. En dehors du fait que les dispositifs légaux «modulation-annualisation » restent à la disposition des acteurs de l'entreprise ou de la branche, la loi sur les 35 heures autorise la réduction de la durée du travail sous forme de jours de repos et s'approche ainsi du principe de l'annualisation (Savel \& Gauthier, 1998, p. 137).

Les solutions envisagées pour atteindre l'objectif de flexibilité varient d'une situation à l'autre: repos compensateur de remplacement pour limiter les heures supplémentaires, temps partiel annualisé, éclatement des deux jours de repos hebdomadaires et consécutifs, temps partiel et horaires variables, semaine de quatre jours comprenant le travail du samedi, réduction du temps de travail pour faire face à la baisse du plan de charges, recours au travail de nuit des femmes pour augmenter la disponibilité de la main-d'œuvre, cette variété de mesures conduit à un cadre temporel éclaté dont les composantes ne forment pas une vision cohérente et ce d'autant moins que ce «temps en mosaïque » s'accompagne d'une remise en cause des repères temporels habituels. On passe (a) d'un repérage temporel hebdomadaire à un repérage annuel, (b) d'un repérage du repos de fin de semaine basé sur deux jours consécutifs à un éclatement et à une délocalisation de cette période de repos, (c) d'une limitation du travail de nuit à son extension aux femmes qui en étaient exclues, (d) d'un repérage en terme d'emploi à temps plein à une vision de l'emploi à temps réduit. La remise en cause des marqueurs temporels habituels est aussi une remise en cause des « normes temporelles du travail», voire des normes de travail. « Au temps de travail taylorien», nous dit Lallement (1993, p. 203) «succède un temps cyclique, perméable aux aléas de la demande sociale et de plus en plus différencié selon les salariés ».

La caractéristique essentielle de ces règles concerne la remise en cause de la stabilité des repères temporels. Les régularités temporelles qui scandent la vie d'un individu (école, 
travail, retraite) sont remises en cause notamment par des périodes de chômage auxquelles sont soumises de nombreuses de personnes, mais aussi par les situations de précarité quelles rencontrent au cours de la vie professionnelle. A cela s'ajoute la dissociation croissante entre, d'un côté, le temps des machines et, de l'autre, le temps des personnes insérées dans des activités multiples. Comme le souligne Boulin (1993, p. 246) : «L'ensemble de ces évolutions montre que les facteurs économiques, sociaux et culturels qui justifiaient une organisation standardisée du travail sont en voie de dilution ».

La remise en cause des régularités est renforcée par la multiplicité des configurations temporelles que l'individu rencontre dans la vie de travail et par les dysharmonies temporelles que ces configurations génèrent dans la vie quotidienne : les repères temporels habituels sont en quelque sorte brouillés. Par repères temporels, on entend les marqueurs qui délimitent et rythment la vie professionnelle et qui déterminent le passage des activités professionnelles aux activités non professionnelles. Sue (1994, p. 13) parle de « dilution des repères temporels ${ }^{\mathrm{a}}$. " Aujourd'hui » dit-il, « ces repères sont brouillés : annualisation, temps partiel, travail de nuit et de fin de semaine $»$.

En résumé, cette présentation du contexte juridique permet de montrer (a) les transformations sur la manière de construire les règles temporelles à partir de 1981, date où le temps de travail fait son entrée dans le champ de la négociation collective, (b) que de nouvelles règles temporelles peuvent alors voir le jour, sous réserve qu'elles combinent temps de travail, flexibilité et emploi, enfin (c) que de tels changements reposent et s'accompagnent d'une autre manière de voir le temps de travail : à la protection du corps des salariés se substitue une conception du temps tournée vers les exigences de la production et de l'emploi au détriment des conditions de travail des salariés.

\section{L'ECLATEMENT DU TEMPS DE TRAVAIL : LA NEGOCIATION ENTRE 1984 ET 1994}

Ces dispositifs juridiques mis en place dans les années 80 et 90 se traduisent par l'ouverture d'un espace de négociation qui était auparavant verrouillé. Le contexte juridique marque la volonté du législateur de laisser aux acteurs de la négociation le soin de trouver des compromis temporels. Il nous reste à vérifier que cette ouverture passe dans les faits et surtout à comprendre la nature des solutions que les acteurs de la négociation vont mettre en place. Bien entendu, d'autres occasions avaient réuni dans un face-à-face employeurs et salariés, mais la question est ici de comprendre comment les syndicats de salariés vont arriver à s'entendre alors même qu'on les sait divisés sur la question du 
niveau de réduction de la durée du travail ? Vont-ils contribuer à la gestion de cet espace de négociation ou bien vont-ils s'en retirer? Quelles sont les configurations temporelles qui se mettent en place? Vont-ils accepter que la réduction de la durée du travail ne soit plus que conditionnelle, c'est-à-dire sous réserve de se soumettre aux exigences de flexibilité ? On sent que se joue ici un tournant dans la négociation dont il s'agit d'évaluer l'amplitude.

Pour cela, nous avons analysé le contenu des accords portant sur le temps de travail et résultant de la négociation collective dans la région Midi-Pyrénées sur une période de 12 ans environ.

En partant de l'analyse des accords sur le temps de travail dans la négociation, on se propose de dégager la diversité des configurations temporelles et leur légitimité. Les accords tirent leur légitimité d'une confrontation entre trois logiques d'action: (1) la logique de l'État qui fixe le cadre légal de la négociation, (2) la logique des employeurs qui cherchent à s'adapter aux variations de l'environnement, enfin (3) la logique des salariés qui, au travers de leurs représentants, tentent de faire valoir leur point de vue. Autrement dit, l'important est moins le résultat que le processus de négociation qui a permis de l'obtenir : il y a bien une action collective qui s'organise autour du temps et des processus de confrontation et de négociation qui se mettent en place. Bien entendu, toutes les négociations ne donnent pas lieu à un accord et de manière symétrique, un accord peut résulter d'un conflit.

L'existence de 680 accords d'entreprise au cours de la période 1984-1994 dans une région témoigne de l'importance du temps de travail dans les négociations (62 accords en moyenne par an). Néanmoins, en 11 ans, il n'y pas d'évolution quantitative alors qu'au niveau national, pour la même période, le nombre d'accords d'entreprises conclus (tous thèmes confondus) double : 3972 accords en 1984 contre 7450 en 1994. De plus, les accords sur le temps de travail représentent 45,1\% des accords (3024) en 1994. Enfin, l'évolution récente montre une augmentation des accords conclus. En 1995, on comptabilise 88 accords sur le temps de travail ce qui signifie une augmentation de $63 \%$ par rapport à 1994 (52 accords).

Il convient de relativiser ces données quantitatives. Tout d'abord parce qu'une entreprise peut regrouper plusieurs établissements et, dans ce cas, un seul accord peut concerner un grand nombre de personnes. Ensuite, l'obligation annuelle de négocier n'est pas une 
obligation de résultat : outre que l'accord peut être reconduit (le thème de la reconduction est présent dans environ un tiers des 680 accords), des quasi-accords ou des désaccords peuvent en résulter, sans pour autant être comptabilisés dans les accords enregistrés. Enfin, les voies de construction de l'accord sont multiples. Une enquête au niveau national auprès de 402 chefs d'entreprise, directeurs de ressources humaines et PDG montre que le renouvellement, la modification ou la conclusion de l'accord d'entreprise ne se fait que dans $56 \%$ des cas avec les délégués du personnel. Un tiers des directions (32\%) négocie avec le comité d'entreprise, $27 \%$ avec les délégués syndicaux et $16 \%$ avec une délégation unique (Lamy social,1995).

\section{II.1. Une forte adhésion aux accords sur le temps de travail}

Lorsque le temps de travail donne lieu à un accord, celui-ci est acquis avec une forte participation des syndicats : sa légitimité est forte. En effet, si l'on considère le taux de présence des syndicats dans l'entreprise et leur taux de signature, on observe une adhésion forte des syndicats aux arrangements temporels élaborés Néanmoins, cette adhésion varie d'un syndicat à l'autre. Chaque organisation syndicale est un interlocuteur potentiel de la direction, mais on voit que la variabilité de leur présence $(11,6 \%$ pour la CFTC et $67,8 \%$ pour la CGT) pèse de manière significative sur leur capacité d'action collective par rapport à l'ensemble des 680 accords de la région. Dans l'hypothèse de la présence des organisations syndicales dans la négociation, on peut constater un taux d'engagement qui oscille entre $86 \%$ et $99 \%$. On est ici dans une logique globale d'adhésion forte des organisations syndicales aux accords négociés ${ }^{16}$. Cette adhésion forte aux accords sur le temps de travail nous conduira ultérieurement à nous interroger : en quoi les organisations syndicales sont-elles ou non cogestionnaires de l'émiettement et de la fin du temps de travail?

\section{II.2. Le temps, monnaie d'échange ou objet de marchandage ?}

L'analyse de 680 accords révèle ensuite que le temps est un objet de négociation souvent associé à la question des salaires ( $42 \%$ des cas) ou à un autre thème (maladie, formation, emploi, classification dans $15 \%$ des cas). Autrement dit, il est traité de façon isolée seulement dans 296 accords, soit 43,5\% des cas (Fig. 2). Ces données pourraient nous amener à conclure que dans les accords sur le temps de travail les négociations sur les salaires pourraient jouer comme mécanisme de compensation. Cette question des

16 Concernant la présence une marge d'erreurs que nous estimons à environ $10 \%$ provient d'une sous-estimation du nombre de délégations syndicales présentes dans l'entreprise au moment de la conclusion de l'accord. Ces effets sont liés à la nature de cette variable dans notre échantillon. 
compensations au sein de la structure externe du temps de travail, sous forme de salaire, d'emploi ou de formation, nous renvoie au termes de l'échange. Pourquoi faudrait-il compenser des compromis sur la structure temporelle par des thèmes de nature différente? Nous verrons par la suite en détail que la composition de la structure interne du temps de travail est tournée vers l'ordre de la production et non vers la vie quotidienne des salariés et elle appelle à d'autres thèmes comme les questions salariales ou les questions de l'emploi pour construire un espace de négociation pertinent.

\section{II.3. Les 6 figures du temps de travail : temps des marchés, temps des machines, temps échangé, temps individualisé, temps des congés et temps confirmés}

Le temps de travail lui-même se présente dans notre échantillon de 680 accords (19841994) comme un objet de négociation extrêmement hétérogène. Nous avons recensé 28 thèmes pouvant faire l'objet d'un accord sur le temps de travail : nous y trouvons aussi bien les «ponts », le chômage partiel ainsi que les thèmes se référant à la flexibilité et à la réduction du temps de travail. Ces thèmes concernent la structure temporelle des activités professionnelle et des congés. Une fois la liste des thèmes établie, nous avons classifié les accords selon la présence de ces thèmes du temps de travail. Notons qu'en moyenne deux thèmes différents composent un accord sur le temps de travail : par exemple, on peut faire un accord qui porte à la fois sur la fixation des dates de congés et sur l'introduction du travail de nuit. La classification des accords selon ces thèmes permet donc de lire les accords différemment. L'objectif est bien d'aboutir à des groupes d'accords se caractérisant par une certaine combinaison de thèmes et qui se distinguent ainsi d'autres groupes d'accords. Après cette classification, nous cherchons à dégager des significations de ces groupes pour le temps de travail. Quelles sont les configurations temporelles qui en résultent? Quelles sont les logiques de négociation qui accompagnent ces configurations temporelles?

Nos 680 accords se retrouvent repartis sur 6 classes d'accords qui se distinguent par le nombre d'accords présents au sein de cette classe et par ses liens différents aux thèmes du temps de travail. 
Les six classes d'accords ${ }^{17}$ se présentent comme suit (Figure 2).

1. La première classe regroupe principalement les accords sur les congés et les ponts (251 accords, soit $37 \%$ ).

2. La seconde se concentre sur la reconduction de la structure temporelle du travail (83 accords, soit $12 \%)$.

3. La classe trois est principalement liée à la modulation de la durée du travail (146 accords, soit $21 \%$ ).

4. La classe quatre regroupe le travail en équipes et de nuit, mais aussi la réduction de la durée du travail (101 accords, soit $15 \%)$.

5. La classe cinq se focalise sur le temps partiel et les horaires variables (77 accords, soit $11 \%)$.

6. La dernière classe regroupe des accords sur des horaires et le repos compensateur (22 accords, soit $3 \%$ ).

\begin{tabular}{|llll|}
\hline classe $1:$ & dates congés payés & dates ponts & congés particuliers \\
251 accords & +++ & +++ & +++ \\
classe $2:$ & reconduction organisation & reconduction durée & repos compensateurs \\
83 accords & +++ & +++ & +++ \\
classe $3:$ & modulation-annualisation & équipes fin de semaine & durée des congés payés \\
146 accords & +++ & +++ & +++ \\
classe $4:$ & travail en équipes & réduction durée & travail de nuit \\
101 accords & +++ & +++ & +++ \\
classe $5:$ & temps partiel & horaires variables & nouveaux horaires \\
77 accords & +++ & +++ & +++ \\
classe $6:$ & travail en équipes & repos compensateurs & travail de nuit \\
22 accords & +++ & +++ & + \\
\hline
\end{tabular}

17 La méthode des classifications (nuées dynamiques) que nous utilisons, calcule pour chaque accord les réponses données aux différentes variables (28 thèmes du temps de travail). Les centres des classes, autour desquels se groupent tous les autres accords en fonction de la similitude des réponses données aux modalités des variables recensées, sont déterminés (jusqu'à la stabilisation) avec un algorithme dynamique. Il s'agit donc d'une méthode de catégorisation des accords qui caractérise à la fois la structure unique de l'arrangement temporel (la classe des accords proches) et l'ensemble des thèmes qu'on y retrouve. Les résultats obtenus révèlent, d'une part, l'existence de 6 classes d'accords distinctes les unes des autres et, d'autre part, des structures internes de chacune des classes, si on considère les thèmes qui caractérisent ces structures internes 
Figure 2. L'éclatement de la négociation du temps de travail en six classes : les accords d'entreprises d'une région (1984-1994)

Ces classes qui confortent l'idée d'une négociation en forme de la structure temporelle du travail autour de pôles (Thoemmes \& de Terssac, 1997a). Sur la base des trois thèmes les plus fortement implantés dans les classes nous voudrions avancer une première interprétation .

Les deux premières classes regroupent en effet l'essentiel des accords sur les congés, les ponts (classe 1) et les reconductions de dispositifs antérieurs (classe 2) : nous interprétons ces classes respectivement comme «le temps des congés», et «le temps confirmé » (Fig. 2). Il pourrait s'agir avec le temps des congés et le temps confirmé de logiques de négociation relevant de la gestion courante du temps de travail en entreprise.

Les quatre classes suivantes touchent à la négociation "extraordinaire » des normes temporelles regroupant l'autre moitié des accords. La classe 4 nous indique que la réduction de la durée du travail ne se présente plus comme un pôle indépendant de la négociation de la structure temporelle. Elle est au contraire intégrée dans un pôle au sein duquel elle semble être échangée contre l'acceptation d'horaires de nuit ou en équipes.

La modulation-annualisation (classe 3) indique le temps des marchés qui décrit l'anticipation les variations de la durée hebdomadaires que le marché suggère à l'entreprise. La durée du temps de travail peut être étendue en période de forte activité, et elle se rétrécit en période basse. Ces variations suivent en quelque sorte les mouvements cycliques des marchés. Cette ainsi que les accords des équipes de fin de semaines montées conjoncturellement dans l'entreprise peuvent se retrouver dans cette classe du temps des marchés.

Les accords qui associent la réduction de la durée du travail à l'aménagement du temps de travail (classe 4) indiqueraient l'existence d'une logique du temps échangé. Au coeur de l'échange, nous trouvons l'acceptation de l'extension de la durée d'utilisation des machines et des services contre une réduction de la durée du travail.

Le temps partiel (classe 5) renvoie à une classe du temps individualisé. Les horaires variables ou individualisés au sein de cette classe qui définissent des plages horaires variables selon les individus conforterait cette lecture d'un «temps individualisé ». Ce 
temps est un temps de contrat individuel (temps partiel) et/ou un temps prenant en compte les effets individuels liés à l'emploi du temps (horaires variables).

Le temps des machines (classe 6) est centré sur les effets liés au travail en équipes et sa compensation. Au coeur, nous voyons le problème de la durée d'utilisation des machines ou services que l'entreprise souhaite accroître.

Ces 6 pôles de négociation sont présentés comme des idéaux-types de la négociation du temps de travail et nous font réfléchir sur ce que recouvrent les dispositifs. Deux de ces 6 pôles portent sur les congés et les reconductions d'accords antérieurs. Observons ensuite que la réduction de la durée du travail n'est pas indépendante des autres thèmes. Le « temps échangé » décrit bien l'échange entre la délocalisation des heures et la réduction du temps de travail. Si ce pôle de la réduction de la durée du travail existe, c'est bien en incorporant la flexibilité du temps de travail.

En somme, on peut indiquer que la majorité des accords (classe 3-6 : 346 accords) est tournée vers l'ordre de la production et vers la flexibilité : le marché, les machines et l'individualisation nous semblent constituer des points d'ancrage forts de ce cadre temporel. Ce qui nous intéresse ce sont les configurations temporelles qui se dessinent. Nous assimilons les six classes à la construction de 6 temps en entreprise : Le temps des congés, le temps confirmé, le temps des marchés, le temps échangé, le temps individualisé et le temps des machines. Ces pôles de la négociation sont aussi des manières de rationaliser le temps et de le décomposer. Le temps de travail paraît ainsi éclaté dans de logiques de négociation multiples, mais en nombre fini. La rationalité dominante de ces accords est celle de la production. 


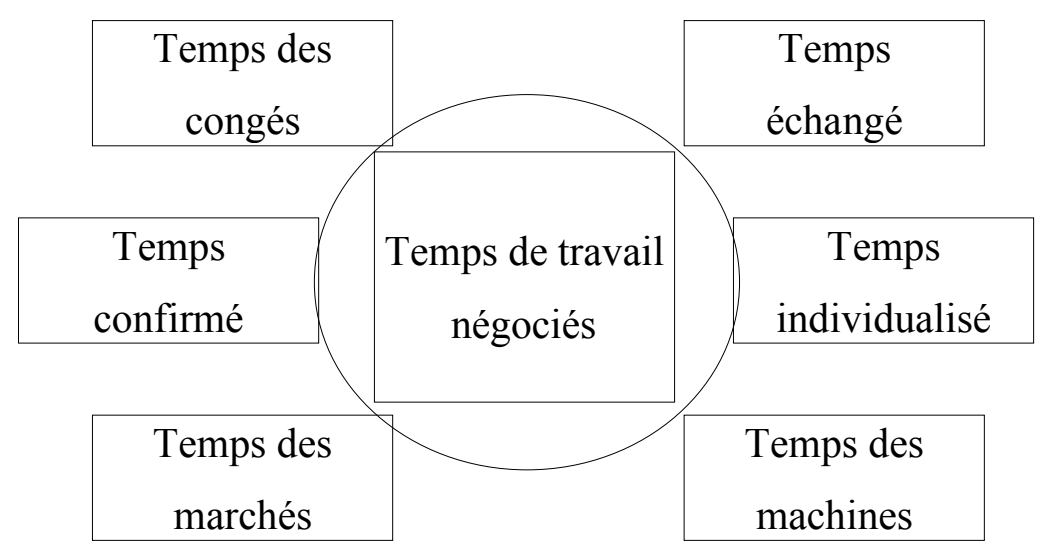

Figure 3 : Les 6 idéaux-types de la négociation du temps de travail dans une région (680 accords 1984-1994)

\section{II.4. Résumé}

Dans cette partie, nous voulions montrer sur quoi porte la négociation du temps de travail. Quels ont été les pôles de la négociation du temps de travail ? Une étude de cas sur les accords d'entreprise dans une région française (entre 1984 et 1994) montre que le temps de travail constitue, en matière de négociation, un objet de recherche complexe. Le temps de travail possède une forte légitimité sociale, car les syndicats signent entre 86 et $99 \%$ des accords. Si l'association de la question des salaires aux négociations demeure importante (42\% des cas), l'éclatement des thèmes du temps de travail s'oppose à la vision simpliste de la traduction du temps en une logique de compensation monétaire et quantitativement mesurable.

Nous montrons au contraire que la logique de négociation est multiple. Elle peut être repartie en 6 idéaux-types de la négociation du temps de travail correspondant chacun à une famille d'accords : le temps des congés, le temps confirmé, le temps des marchés, le temps individualisé, le temps des machines, le temps échangé. L'étude des négociations autour des 680 accords entre directions d'entreprises et organisations syndicales sur 11 ans dans une région montre la multiplicité des configurations temporelles à l'intérieur même du champ du temps de travail. La proposition d'une lecture des significations de ces arrangements par une classification que nous interprétons autour de 6 idéaux-types a pour 
but de montrer que sous le désordre apparaît un éclatement orienté selon différentes formes temporelles de flexibilité. L'existence de 4 pôles de négociation autour de la modulation, du temps partiel, du travail en équipes, du travail de nuit et de la réduction de la durée du travail nous montre un espace de négociation en plein changement au cours de la décennie 1984-1994 : l'ordre temporel dominant concernant le temps de travail s'établit sur la logique de la production, au détriment de la logique de la vie quotidienne des salariés ; c'est ce que nous allons approfondir maintenant.

\section{VERS L'INTENSIFICATION DISCONTINUE DU TRAVAIL?}

La loi de Juillet 1994 concernant le Compte Epargne Temps (CET) va permettre d'augmenter la flexibilité dans la gestion du temps de travail, puisqu'elle autorise une gestion pluriannuelle des congés. Le dernier rempart de la stabilité du temps de travail se trouve donc fissuré. Peut-on cependant considérer cette loi comme un dispositif, parmi d'autres, favorisant la flexibilité de la production? Ou au contraire, peut-on interpréter le CET comme un dispositif favorable au salarié du seul fait qu'il l'autorise à épargner du temps ou de l'argent afin de financer un congé de longue durée ? Le fait que ce dispositif soit facultatif et que son utilisation ne résulte que de la décision de l'individu le rend-il plus légitime?

Nous ne caractériserons pas ici les formes de mise en œuvre de la loi (de Terssac, Thoemmes, Flautre, Thébault, 1998a), mais plutôt les formes d'utilisation des accords négociés : d'un côté, nous considérons que le CET n'acquiert sa signification que dans le contexte dans lequel il se développe et de l'autre, nous pensons que sa signification se construit aussi dans les formes d'utilisation de ces accords. D'un côté, les producteurs sont tous ceux qui sont associés à cette production normative : organisations syndicales et employeurs ; d'un autre côté, les utilisateurs sont ceux qui décident d'ouvrir un compte, voire de consommer le temps qui a été épargné. Nous avons réuni un ensemble d'accords de branche et d'entreprise concernant le compte épargne temps. Notre objectif est de comprendre à partir de ce qui est mis par écrit dans les accords, quelles conceptions du temps de travail sont véhiculées au travers de ce dispositif. Sans préjuger des usages faits de ce dispositif par les acteurs dans l'entreprise nous tentons d'isoler quelques éléments qu'apporte l'accord à la définition du cadre temporel des activités : conçu en premier lieu pour mieux gérer les activités, le compte-épargne-temps est une « soupape » qui permet de réintroduire le projet de l'individu face à un temps de travail de plus en plus contraignant. 
Mais simultanément ce compte permet la pluriannualisation des activités. L'individu peut tourner cet échange a son profit. Mais d'une part cet échange est placé dans un cadre de dépendance du contrôle hiérarchique (donc c'est de l'autonomie contrôlée) et d'autre part, cet échange comporte la possibilité d'une intensification du travail par le report de repos et congés.

\section{III.1. Le compte épargne temps (CET) : une loi pour mieux gérer les activités}

Peut-on considérer que les règles temporelles actuelles ne sont tournées que vers la production? L'ordre temporel serait-il inéluctablement guidé par la production ? L'analyse détaillée des règles temporelles nous montre qu'elles peuvent être orientées vers la vie quotidienne des salariés et c'est dans cette perspective que nous abordons les règles temporelles touchant le Compte Epargne Temps (CET) : dans la multitude des règles tournées vers la production, elles font exception. De quoi s'agit-il ? D'un dispositif récent (loi de 1994) qui permet au salarié d'épargner au cours de sa carrière du temps qu'il pourra consommer ultérieurement sous forme de congés. La spécificité de ce dispositif est double : d'une part, il permet au salarié de se constituer un «capital-temps »; d'autre part, la gestion de ce « capital-temps » est, dans le cadre d'un accord de branche ou d'entreprise, à l'initiative du salarié. À la différence des autres dispositifs, c'est le salarié seul qui décide d'avoir (ou non) recours à cette procédure, c'est-à-dire d'ouvrir le compte, de l'alimenter et de consommer ce capital-temps en cours ou en fin de carrière : le CET est en quelque sorte sous le contrôle exclusif de son bénéficiaire. Si une partie de la gestion du temps passe du côté du salarié, reste à comprendre comment cette « délégation » s'articule avec le reste des temporalités orientées vers la production qui, elles, sont gérées « d'en haut »et «de l'extérieur» pour le salarié. Notons simplement ici l'existence d'une délégation d'initiatives individuelles dans un ensemble de règles temporelles collectives et imposées à tous, une fois négociées.

\section{III.2. Le CET comme " soupape " et comme cadre temporel pluriannuel}

Le conflit de rationalités (production et vie quotidienne) se déplace vers le salarié et se transforme, d'une part, en un conflit de disponibilités entre vie professionnelle et vie non professionnelle, et, d'autre part, en un conflit situé sur un même horizon temporel (court terme) que le recours au CET servira à gérer du fait de son rôle de "soupape », (de Terssac, Thoemmes, Flautre, Thébault, 1998b). 
- Premièrement le conflit entre rationalités se déplace vers le salarié, car c'est lui qui devra le gérer : dès lors que la règle temporelle est entièrement tournée vers la flexibilité de la production (modulation, annualisation, délocalisation des horaires de travail, changement du statut d'emploi à temps réduit, etc.), il exige de la part du salarié une disponibilité professionnelle renforcée.

- Deuxièmement, le conflit se transforme en conflit de disponibilités, car l'extension de la disponibilité non-professionnelle est de plus en conflit avec la disponibilité professionnelle. Le salarié cherche de plus en plus de temps consacré à ses activités non professionnelles. En effet, c'est bien à l'extension de la disponibilité non-professionnelle du salarié à laquelle on assiste. Le salarié voit augmenter son temps non-affecté à ses activités professionnelles: d'un côté, il y a bien une augmentation des congés et des périodes de repos en échange de l'acceptation de la disponibilité productive ; de l'autre, on assiste bien à une augmentation du temps non-professionnel du fait des diverses procédures de conversion d'aspects financiers (primes, heures supplémentaires, etc.) en temps. D'où un véritable conflit pour une période de temps donnée (en général l'année) entre la disponibilité professionnelle requise et la disponibilité non-professionnelle acquise.

- Troisièmement, le conflit entre la disponibilité requise et la disponibilité acquise peut être atténué par la négociation sur le temps de travail si celle-ci intègre le recours au Compte Epargne Temps. En effet, le recours au CET va permettre d'allonger l'horizon temporel et de mettre en place une gestion pluriannuelle du temps. De ce fait, le recours au CET va donner la possibilité, d'un côté, d'épargner à court terme de la disponibilité nonprofessionnelle en constituant un « capital temps », et, de l'autre côté, de disposer de cette disponibilité à moyen ou à long terme. En ce sens-là, le CET pourrait jouer un rôle de «soupape » temporelle : il permet de gérer le conflit de rationalités production - vie quotidienne, car le salarié ne peut pas en même temps être disponible pour la production et pour ses activités non professionnelles

\section{III.3. L'émergence d'un temps virtuel}

Le CET introduit un double changement des termes de l'échange entre les salariés et leur employeur : d'un côté, les salariés acceptent l'extension des dispositifs orientés vers la flexibilité en échange des mesures de compensation : le temps «dépensé » dans les conditions de la flexibilité est « regagné » pour les activités non professionnelles. D'un autre côté, les salariés acceptent que le temps « regagné » ne puisse être consommé par le 
salarié au moment où il le décide, en échange de la possibilité de l'épargner et de le consommer dans un temps différé (op. cité).

On observera premièrement que, dans cet échange, l'autonomie des salariés se trouve fortement encadrée, puisque la décision de consommer le temps épargné est soumise à l'appréciation de l'encadrement : il ne s'agit donc pas d'autonomie, puisqu'elle s'exerce dans un cadre de dépendance et dans un cadre d'initiatives contrôlé par le supérieur hiérarchique. Néanmoins, le salarié a la possibilité dans le cadre des accords, d'ouvrir un compte et donc d'épargner du temps.

On observera deuxièmement que, dans cet échange, le temps de la vie quotidienne se dédouble en un temps virtuel dont la consommation est différée. Le temps présent qui pourrait être consommé pour des congés ou des repos, devient un temps abstrait qui peut être extrait du contexte réel et reporté à plus tard. Ce dédoublement du temps de la vie quotidienne, en un temps présent et en un temps futur, fait émerger un « temps virtuel». Il n'y a pas de matérialité, puisqu'il est transformé en un segment vide de contenu et décontextualisé. Cependant il peut être rendu à la réalité, lorsqu'il est consommé : mais cette consommation se déroule dans un contexte différent.

Autrement dit, et c'est notre troisième observation, on assiste à l'émergence d'un temps à la carte que l'on peut dissocier du contexte dans lequel et pour lequel il a été acquis, que l'on peut extraire pour un temps de la vie réelle des individus. Le temps à la carte repose sur une conception du temps abstrait, vide de contenu, composé de segments temporels comparables entre eux, comme si les jours de repos qui viennent adoucir la vie quotidienne ou les jours de congés qui permettent de s'ajuster aux temporalités sociales, étaient équivalents à un départ anticipé à la retraite.

Pour autant ces observations n'enlèvent rien à la portée du dispositif qui consacre la flexibilité et la délocalisation du temps consacré aux activités professionnelles, temps qui ne cesse de croître.

\section{III.4. Le CET et la flexibilité temporelle}

L'analyse des accords effectuée dans 35 entreprises montre que le CET n'est pas un dispositif isolable des autres mesures temporelles. Nous avons montré l'existence de trois types d'enchaînements dans les accords qui englobent le CET : 
- l'enchaînement « linéaire » de l'accord reproduit un accord CET ultérieurement ou dans un autre site,

- l'enchaînement « indépendant », ou « combiné » utilise l'accord CET comme dispositif complémentaire d'autres dispositifs temporel qui peuvent être liés à la flexibilité,

- le CET « intégré » dans la flexibilité productive : tout d'abord, parce qu'incorporés dans les accords sur le temps de travail liés aux thèmes de la flexibilité ; ensuite parce que l'analyse des textes d'accords indique que certains thèmes que nous avons appelés des « charnières temporelles», font basculer le CET dans des dispositions favorisant la flexibilité de la production et visant à augmenter la disponibilité du salarié pour son entreprise. Par exemple la liaison intégrée travail en équipes- repos compensateurs et compte épargne temps peut aboutir au report de tous repos issu des effets liés au travail en équipes. Le repos est différé et non consommé au moment où prévaut encore la fatigue liée au travail en équipes. L'accumulation des congés issus ou non d'une réduction de la durée $\mathrm{du}$ travail sur un compte épargne temps peut aussi témoigner d'une intensification potentielle du travail dans une entreprise sur des périodes données.

Cela signifie que le CET n'acquière toute sa signification que par le contexte dans lequel il a été négocié.

Au total, le CET apparaît comme un dispositif complémentaire des autres dispositifs relatifs au temps de travail. Il favorise l'émergence d'un «temps virtuel» et oblige à s'interroger sur l'intensification potentielle du travail qui pourrait résulter de cette possibilité de différer ses repos ou congés.

\section{DisCUSSION ET CONCLUSION}

\section{IV.1. La perte de centralité de la durée du travail}

Il y a bien une perte de la centralité de la durée hebdomadaire du travail au profit d'autres composantes du temps de travail, relatives à la répartition des temporalités journalières ou annuelles.

Ces nouvelles figures temporelles apparaissent dans un contexte juridique ouvrant de nouveaux espaces aux négociateurs : le tournant de 1981 ouvre un espace de négociation du temps de travail en même temps qu'il délimite cet espace en incitant les négociateurs à se ranger dans le triangle : durée, flexibilité et emploi. La transformation de fond concerne 
le statut de la durée du travail qui devient un objet de marchandage contre l'acceptation de la flexibilité et la prise en compte des questions de l'emploi. Il conviendra d'évaluer la nature et l'importance de la prise en compte de l'emploi dans les négociations. S'agit-il seulement de maintenir l'emploi ou bien d'en créer de nouveaux? S'agit-il d'un engagement ferme ou d'une simple déclaration d'intentions.

\section{IV.2. Les figures récentes du temps de travail sont orientées vers la logique de la production}

L'espace de négociations ainsi ouvert est largement occupé par la négociation sur le temps de travail, tant au plan régional qu'au plan national. Notons cependant qu'existent des différences entre le niveau régional et le niveau national : en particulier notre échantillon régional laisse apparaître une plus grande adhésion de certaines organisations syndicales à ces accords. Peut-être est-ce dû à la nature des matériaux sur lesquels nous avons travaillé, et qui sont d'une fiabilité relative quant à l'inscription des syndicats présents ? On peut penser aussi que la négociation étant aussi une négociation touchant les salaires (43\% des cas), cela entraîne une plus forte adhésion de l'ensemble des syndicats.

Ce qui frappe, c'est que le temps de travail ne donne plus lieu à des négociations concernant exclusivement les normes temporelles, c'est-à-dire la durée journalière et hebdomadaire, les repos et les congés. Ces accords laissent apparaître l'inclusion dans la négociation de thèmes qui sont d'une part, externes à la structure temporelle (comme l'emploi et les salaires) et qui sont d'autre part, internes au temps de travail. Observons que les paramètres qui interviennent dans la négociation, pour définir le temps de travail, sont nombreux (28 thèmes au total). Dans chacun des accords, on observe qu'il $\mathrm{y}$ a deux thèmes en moyenne se rapportant au temps de travail : cela interdit de ranger un accord sous une seule rubrique.

Au-delà de la variété des thèmes, l'analyse des pivots de la négociation nous a permis de montrer que la négociation collective s'organise autour de 6 temps, que nous avons interprété comme des idéaux-types. Si les deux premiers temps, le temps des congés et le temps confirmé, apparaissent comme deux pivots habituels de la négociation, cela n'est pas le cas des autres. Le « temps des marchés », le «temps individualisé » et le « temps des machines » indiquent bien le tournant dans la négociation: les exigences de la production sont omniprésentes dans le temps de travail. Le temps « échangé » atteste que l'on ne traite plus de la durée du travail en elle-même : la perte de la centralité de la durée est consommée dans les pratiques. Bien entendu, il nous faudra approfondir le contenu et 
les enjeux de ces divers temps, en particulier le temps "individualisé »: il ne s'agit pas d'un temps choisi, mais plutôt de temps partiel imposé ; dès lors la question du statut de l'emploi ne peut plus être traitée en dehors du temps de travail, qui distribue les populations de manière différentielle au regard du statut de l'emploi.

Nous proposons d'interpréter les résultats de la négociation collective, comme le triomphe de la logique de la production, qui impose désormais sa loi sur la définition de la structure temporelle de travail. Cela donne une autre signification au temps de travail, dont on peut se demander s'il ne devient pas, soit le temps de la production, soit le temps de l'emploi, soit les deux. A ce stade de l'analyse, il ne peut s'agir que d'une hypothèse interprétative qui reste à vérifier. Néanmoins, ces figures temporelles ne sont pas décrétées «d'en haut »; elles ne sont pas non plus imposées par l'intervention unique de l'État. Elles sont bien obtenues avec l'assentiment des syndicats, du moins lorsqu'ils signent les accords.

\section{IV.3. De l'éclatement à l'intensification discontinue du travail ?}

L’interprétation que nous venons de proposer est lourde de conséquences : elle signifie que le temps de la vie quotidienne est désormais sous l'emprise du temps orienté vers la logique de la production. Qu'est-ce que cela veut dire ? Si l'on songe à la construction de l'ordre temporel entre 1830 et 1980, on peut dire que le temps de travail fixe la frontière entre le temps consacré aux activités professionnelles et le temps consacré aux activités non professionnelles: d'un côté la durée journalière et hebdomadaire, ainsi que leur répartition, de l'autre les repos et les congés. Cette norme temporelle a constitué un enjeu très disputé, avec au centre la durée du travail, que les uns veulent voir augmenter et que les autres tentent de faire diminuer. Certes, la présentation est un peu simpliste, si l'on considère que les temps situés en dehors des activités professionnelles, peuvent aussi contenir des activités de travail, domestique, par exemple. Mais cela signifie, qu'il y avait, jusqu'à une période récente, une relative stabilité de la structure temporelle.

Cet enjeu n'a pas disparu, mais il se pose en d'autres termes, car la frontière devient « virtuelle», non qu'elle n'existe plus, mais elle devient floue: d'abord, et comme antérieurement, il y a bien une différence entre la durée officielle et la durée effective ; ensuite parce qu'aucune des composantes temporelles n'est stable : ni au plan des durées, ni au plan des horaires, ni au niveau des statuts d'emploi. Même si la durée hebdomadaire du travail diminue, cela est difficile à percevoir pour l'individu qui voit grandir la variabilité et l'instabilité de ses conditions temporelles de travail ; de telles fluctuations, 
n'entrent-elles pas en conflit avec les temporalités des autres personnes avec qui chacun est en interaction?

La baisse d'importance de la logique de la vie quotidienne résulte, selon nous, du fait que les marges de manœuvre pour faire valoir les temps non professionnels sont réduites : sans doute que les menaces sur l'emploi l'emportent. Mais cela nous oblige à nous interroger sur deux aspects de la structure temporelle actuelle. Premièrement, la négociation sur le temps de travail peut être déclenchée par les employeurs, comme par les salariés, qui de ce point de vue gardent leurs marges d'initiatives; mais toute négociation est désormais « encapsulée » dans le triangle flexibilité-emploi-durée. Deuxièmement, les configurations temporelles amènent les négociateurs à construire des solutions qui visent à s'adapter aux temps de la production, sans forcément mesurer les conséquences sur la santé des salariés. On observera tout d'abord, que les discussions sur le temps de travail ne portent que sur le cadre temporel, dont sont absentes les activités qui s'y déroulent; de plus les cadres temporels ainsi définis, contraignent le salarié à se soumettre aux exigences de la production : il doit désormais fournir plus d'efforts, pour satisfaire les exigences de la production, quitte même à ne pas prendre ses congés, voire ses repos, au moment opportun pour lui. Autrement dit, ne perd-il pas le contrôle de la gestion de son temps personnel, du fait que ses prises de congés et de repos sont fortement soumises au ralentissement de la production ou à l'arrêt des machines ?

C'est ce que nous avons montré avec le Compte Epargne Temps. Cette loi a été élaborée pour au moins deux raisons : (a) des difficultés de report de congés d'une année sur l'autre, (b) une volonté d'organiser le temps de travail sur toute la vie pour certains syndicats. L'examen de dix conventions collectives de branche montre que le Compte Epargne Temps permet de résoudre un conflit de disponibilités entre le temps pour l'entreprise et le temps pour soi. Dès lors, il devient un outil de gestion complémentaire pour permettre à l'employeur d'avoir des salariés disponibles en fonction des exigences de la production et au salarié de ne prétendre à utiliser ses droits aux congés et aux repos, sous couvert qu'il peut les épargner.

L'ordre temporel repose plus que jamais sur le temps de travail comme temps dominant l'organisation de toutes ses activités et ce, d'autant plus que le salarié perd la maîtrise de la gestion de son temps personnel. Il reste à comprendre plus précisément pourquoi les salariés donnent leur assentiment à des solutions qui ne paraissent pas leur être totalement favorables. C'est pourquoi, nous analyserons les modalités concrètes par lesquelles il faut 
passer pour aboutir à ces nouvelles solutions. L'analyse monographique que nous présentons maintenant, montre que les solutions pratiquées sont loin de s'imposer mécaniquement, même si la question de l'emploi traverse les situations étudiées. 



\section{CHAPITRE III}

LES ARRANGEMENTS TEMPORELS NEGOCIES 


\section{Introduction}

Le temps réglementaire est désormais " éclaté », puisque la structure temporelle basée sur des normes, sur l'intervention de l'État, sur des règles communes et sur des dispositifs de sanction vole en éclats. Au cours du chapitre précédent, nous avons montré qu'un nouvel espace de négociation du temps de travail s'ouvre: le législateur demande aux négociateurs de produire des accords qui combinent la durée du travail, la flexibilité et l'emploi. C'est bien ce que font les acteurs de la négociation en produisant une variété de configurations temporelles que nous avons interprétées comme le triomphe de la logique de la production. Cette domination des exigences de la production sur l'organisation du temps de travail s'est révélée au travers de quatre figures temporelles appelées : « le temps échangé », "le temps individualisé », «le temps des machines» et «le temps des marchés ». La conséquence de la prédominance de cette logique de la production est que les dispositifs élaborés par le législateur concernant le temps personnel des salariés (comme le Compte Epargne Temps) peuvent être utilisés dans les accords de branche pour rendre le salarié encore plus disponible à l'égard de l'entreprise : on demande désormais au salarié de prendre ses congés lorsque les exigences de la production le permettent.

Comment les organisations syndicales des salariés ont-elles pu «s'aligner» sur cette logique de la production au détriment de la logique de la vie quotidienne des salariés?

Un premier élément de réponse a été donné lorsque nous avons montré que le temps de travail a tendance à devenir le temps de l'emploi. Néanmoins, il ne faudrait pas conclure trop hâtivement à une subordination du comportement syndical aux contraintes des systèmes de production. L'analyse concrète effectuée en entreprise, au travers de monographies, devrait nous permettre de comprendre comment les acteurs de la négociation «s'arrangent» pour trouver des formes temporelles dans des situations locales. L'objet de ce chapitre concerne donc la construction de ces «arrangements temporels ». Après l'invention et l'éclatement du temps de travail, nous cherchons à comprendre le processus de la reconstruction des cadres temporels. Dans notre perspective, il ne s'agit pas avec l'éclatement du temps de travail d'une déréglementation ou d'une dérégulation des normes. L'éclatement donne lieu à des configurations différentes du temps de travail. Les règles et régulations du temps de travail se recomposent.

Il s'agit de comprendre plus précisément en quoi la négociation du temps de travail, qui combine un mode d'intervention spécifique de l'État et une mobilisation des acteurs, peut conduire à la production d'un cadre temporel de travail légitime pour chacune des parties 
engagées dans la négociation. La question n'est pas seulement de savoir si les acteurs sont capables de coopérer alors que le temps de travail les divise et les oppose. Il s'agit de comprendre aussi les conditions de la négociation elle-même et avant tout l'objet de la négociation. Le temps de la production, abstrait et vide de contenu, peut-il être confronté aux divers temps avec lesquels les individus partagent leur existence? Autrement dit, l'analyse de la négociation du temps de travail ne doit pas présupposer son objet, ni le tenir pour acquis: l'analyse doit le construire. C'est pourquoi nous proposons d'utiliser la notion « d'arrangement temporel ». D'un côté, cette notion désigne un compromis dont le résultat est fragile; de l'autre, elle désigne un accord non seulement sur une solution locale, mais aussi sur la réalité du temps qui est négociée.

La notion «d'arrangement » comporte deux idées principales (Dictionnaire Petit Robert, 1989, p. 103) : (a) une idée d'ordre puisqu'il s'agit de « disposer les choses dans un certain ordre », de les agencer et de les aménager correctement ou d'une manière choisie ; (b) une idée d'accord puisque l'arrangement vise aussi les mesures qui ont été prises pour « régler un différend par une convention, un compromis, une conciliation ». La notion « d'arrangement temporel» désigne donc, d'une part, la nature des solutions temporelles élaborées qui renvoient à l'ordre des actions (notamment de travail) telles qu'elles sont structurées par le temps ; elle désigne, d'autre part, la manière dont ces solutions ont été construites, ce qui renvoie à la négociation conduisant à des compromis.

Compte tenu de la définition proposée, «l'arrangement temporel» revêt deux caractéristiques essentielles qu'il convient d'explorer. Tout d'abord, il y a une grande variété «d'arrangements temporels» et il convient donc de rechercher une pluralité de solutions imaginées. Ensuite, la notion «d'arrangement temporel», en tant que compromis, combine des éléments temporels différents et cristallise des choix qui ont été pris à des niveaux bien différents : la nation, la branche, l'entreprise, voire l'individu, qui constituent autant de lieux d'élaboration de compromis à analyser.

La négociation produit des arrangements temporels localisés: ils proviennent de confrontations et de compromis entre les protagonistes, à propos d'un cadre temporel ; ce cadre temporel porte la marque des disputes autour du triptyque flexibilité-emploi-durée. Si l'emploi constitue le fil conducteur de ces discussions, il n'en reste pas moins que le résultat combine, d'une part, l'articulation entre des temporalités multiples (professionnelles et non professionnelles) et la pression temporelle qui s'exerce dans le travail. Il combine, d'autre part, les représentations et les conceptions qu'ont les 
protagonistes du temps de travail et dont nous voudrions expliciter la composante sexuée. En effet, nous considérons que le temps de travail n'échappe pas aux visions sexuées que véhiculent les négociateurs. C'est la raison pour laquelle nous proposons d'explorer les chemins de la négociation pour bien montrer la complexité et les difficultés à parvenir à un accord qui reste provisoire et local. La fragilité des configurations temporelles, si elle était vérifiée, donnerait une autre signification au temps de travail: non seulement variable d'une situation à l'autre, voire d'un individu à l'autre, mais aussi instable, transitoire et sans cesse rediscutée. Dans une première partie, nous cherchons à comprendre la construction et le sens des arrangements temporels : le temps échangé (flexibilité contre réduction), le temps individualisé, le temps des machines et le temps des marchés sont-ils imperméables aux rapports sociaux? Nous voudrions montrer que les régulations partielles du temps de travail portent sur des questions de volumes d'emplois, de statut d'emploi, de visions sexuées du travail, sur la compatibilité des emplois du temps et des horizons temporels. Dans une seconde partie, nous cherchons à rendre compte plus en détail du passage du temps de travail au temps de l'emploi qui nous semble central dans la construction des arrangements temporels. Nous verrons que ce passage est caractérisé par l'hétérogénéité des régulations partielles et locales. Trois formes se dégagent des enquêtes de terrains : l'emploi-contrainte, l'emploi-catalyseur et l'emploi-écran de la négociation du temps de travail. L'éclatement du temps de travail s'accompagne d'une reconstruction locale des normes. Celle-ci rend compte des divisions sociales à propos du temps de travail. De l'autre côté, le compromis ou l'arrangement fait intervenir une autre question qui est celle de l'emploi et dont la signification est multiple.

Nos monographies portent sur des accords d'entreprise conclus au cours des années 1994 et 1996. Partant des accords écrits, nous avons tenté de les situer par rapport à l'ensemble des accords conclus sur le temps de travail dans l'entreprise. Ensuite, nous avons tenté de comprendre avec nos interlocuteurs la signification du processus de négociation qui aboutit à un moment donné à un résultat provisoire. Cette démarche a été utilisée pour les 11 établissements.

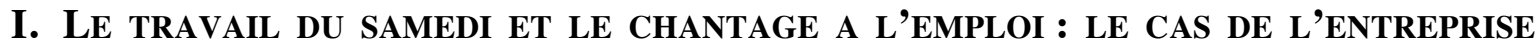

\section{CLARO}

Nous voudrions montrer avec ce cas un exemple pour la construction et le sens que peut prendre le «temps échangé ». Nous sommes en effet en présence d'un accord qui échange 
flexibilité contre réduction de la durée du travail. L'échange prend ici la forme du chantage ou de la menace, car le spectre des licenciements hante les acteurs de la négociation. L'intérêt particulier de ce cas constitue la manière dont les acteurs « gèrent » la menace du chômage dans le contexte local d'une négociation historiquement conflictuelle du temps de travail.

Dans l'entreprise «CLARO» du secteur de la métallurgie (1300 salariés) ${ }^{18}$, un accord d'entreprise vient en 1996 de régulariser le travail du samedi. Cet accord porte sur la réduction de la durée du travail et la suppression d'un plan social prévoyant plus d'une centaine de licenciements. Il stipule en échange «d'augmenter le temps d'utilisation des équipements de production » (préambule, $1 \mathrm{~b}$ ). Le compromis trouvé porte sur la réduction de la durée du travail de 38 h 30 à 37 h par semaine. Pour les équipes de jour, une semaine de 4 jours est introduite, soit 4 × 9 h $15=37 \mathrm{~h}$ par semaine. La perte salariale atteint environ 3,4\%. Le travail de samedi devient régulier une fois toutes les trois semaines. Cet accord a été signé par l'employeur, la CFDT et $\mathrm{FO}^{19}$, mais pas par la CGT. La principale mesure concerne le travail du samedi contre lequel les syndicats se sont battus pendant plus de dix ans. L'importance de cet accord réside non seulement dans son contenu, mais aussi, comme nous allons le montrer, dans l'histoire de sa construction et dans les termes de l'échange qui ont été modifiés par cette histoire.

\section{l.1. Blocage dans la négociation au regard du travail du samedi}

L'entreprise «CLARO » est installée depuis plus de 25 ans dans la région et connait une longue tradition de négociations sur la réduction et l'aménagement du temps de travail, marquée par une série d'échecs.

Ces négociations successives ont été à l'initiative de la direction en 1982, 1984, 1989 et 1995 ; elles se soldent régulièrement par des échecs que nous allons illustrer par trois exemples.

Un premier exemple de blocage nous est fourni à propos de la crainte des salariés de voir leur pouvoir d'achat diminuer. En 1982 et 1984, la direction propose, dans un contexte économique de stagnation, une réduction de la durée du travail compensée partiellement. Ces négociations sont toujours refusées par les organisations syndicales opposées à une perte salariale.

18 La répartition selon le bilan social de 1995 est de 200 femmes et de 1100 hommes.

${ }^{19}$ Le sigle exact est CGT-FO (Confédération générale du travail - Force Ouvrière) 
Un deuxième exemple nous est fourni par la négociation visant à promouvoir le travail le samedi. A partir de 1989, le changement de la conjoncture économique amène la direction à proposer une réduction de la durée du travail compensée quasiment totalement, mais elle souhaite, en contrepartie, introduire un travail régulier le samedi : "Comme la demande était très forte, nous avons demandé aux syndicats de négocier une réduction de la durée du travail pour essayer d'utiliser les installations au maximum du lundi matin $5 \mathrm{~h} 48$ au samedi soir 22 h $12 »$, confie le responsable de la direction. Cette proposition est encore refusée par les syndicats. Ce refus constitue aussi un échec pour les organisations syndicales, car elles ne savent pas comment répondre à la riposte de l'employeur qui, lui, commence à embaucher du personnel à temps partiel et ainsi oblige les nouveaux arrivants à travailler le samedi par contrat. ${ }^{20}$.

Un troisième exemple de blocage voit le jour en 1995. La proposition d'une réduction de la durée du travail (avec une compensation financière à $60 \%$ ) est renouvelée dans un contexte d'arrêt de la fabrication d'un produit. Les syndicats se retirent de la négociation en refusant le travail du samedi soir qui était proposé comme une contrepartie de la réduction de la durée du travail. «La direction nous a amené à ces négociations en parlant de réduction de la durée du travail, du temps de travail officiel, mais pour la direction l'aspect principal de l'accord, c'est l'aménagement, c'est ce qu'elle recherche avant tout » (responsable CGT).

\section{I.2. La négociation de 1995 et 1996 : les syndicats changent de position par rapport au travail du samedi}

Nous allons rendre compte, ici, des diverses positions qui interviennent dans les négociations de 1995 et 1996. Autrement dit, nous verrons comment les positions des divers acteurs de la négociation vont se modifier au gré du déplacement de l'objet de la négociation et comment les alliances se construisent et se défont dans le temps. Nous allons distinguer deux phases dans la négociation: la première phase a consisté pour certaines organisations syndicales à bloquer le processus de négociation; la deuxième phase a consisté à débloquer la négociation par une menace de licenciements de la part de la direction.

20 Le nombre global des temps partiels atteint 50 salariés (dont 15 hommes), comme le note le rapport sur l'égalité professionnelle de 1995. 


\section{I.2.A. Le blocage de la négociation par le recours au référendum}

La première phase rend compte du blocage de la négociation par le recours à des référendums organisés par la CGT. Nous identifions trois étapes.

- Premièrement, face à la concurrence accrue dans le secteur et à l'arrêt d'un produit en 1995, FO, la CFDT et la direction entrent en négociation pour essayer d'aménager le travail le samedi; les termes de l'échange étaient : réduction de la durée du travail contre modulation.

- Deuxièmement, la CGT se positionne contre toute forme de modulation, comme le note un responsable CGT : «La différence est qu'en 1982, 1984 et 1989, la CGT refusait absolument de négocier y compris sur ce qu'on appelait la modulation, l'aménagement et la flexibilité ». Cette position de principe les conduit à évaluer auprès des militants puis de l'ensemble des salariés la pertinence du refus du travail du samedi, car pour la CGT «les gens ne voulaient pas sacrifier le samedi après-midi. [...] La vie familiale, c'est le samedi et le dimanche. Moi, je suis un militant parent d'élève, on s'est battu pour que les gosses n'aillent pas à l'école le samedi et, là maintenant, je vais partir, je vais laisser mes gosses à la maison ». L'argumentation concerne la concordance des temps entre les activités professionnelles et les activités non professionnelles. La première consultation des adhérents aboutit à la conclusion d'une acceptation du travail exclusivement le samedi matin, ce qui était insuffisant pour la direction qui souhaitait un travail régulier tout au long du samedi après-midi et soir. Le deuxième référendum organisé par la CGT concerne tous les salariés. Il porte sur la question du samedi après midi : $66 \%$ des salariés refusent que les syndicats négocient sur le travail du samedi et ils s'expriment contre un mandat « de négociation du travail du week-end » pour les organisations syndicales.

- Troisièmement, dans ces conditions, les syndicats CFDT et FO se retirent de la négociation et le blocage est consommé. A cela, il faudrait ajouter les nombreuses actions collectives que résume un responsable CGT : «Il y a eu plusieurs arrêts de travail. En décembre; il y a eu des actions spontanées, il y en a eu au sein de l'entreprise. C'est quelque chose d'assez exceptionnel, il y a des gens qui sont allés manifester devant le $\mathrm{CCE}$, devant le bureau social, c'est la première fois que je vois ça dans l'usine. Le directeur est descendu et s'est expliqué devant les grévistes pour dire ce qu'il fallait faire et pourquoi. [...]. La pression était forte au niveau de la direction, le message était : ce n'est plus du chantage, c'est de la réalité. Et nous, notre message consistait à dire : allons contre 
la réduction de la durée du travail sans parler du plan social \{les licenciements\}; s'il y a un plan social, on verra bien, il est toujours temps de réagir à ce moment-là ».

\section{I.2.B. L'intervention du plan social pour débloquer la négociation}

La seconde phase intervient dans un climat de réduction des effectifs de $10 \%$. Le plan social est alors discuté lors de réunions du CE pour tenter de trouver des compromis malgré les divergences affichées, comme en témoigne ces échanges entre la direction et la CGT.

- Responsable de la direction: «Je ne peux plus prendre du recul; je considère que l'aménagement et la réduction de la durée du travail n'a pas abouti, c'est pourquoi nous devons continuer cette réunion en parlant du plan social et nous devons également suivre le règlement à appliquer $»$.

- Responsable CGT: «Vous ne pouvez pas supposer que la CGT signera l'accord, mais un accord est encore possible ».

- Responsable de la direction: «Il y a des doutes sur la possibilité de cet accord et nous sommes assez loin les uns des autres $»$.

- Responsable CGT: « Vous voulez dire qu'il y a alors désaccord total ».

- Responsable de la direction : « [...]. J'ai fait des propositions pour tenter de régler les problèmes, je ne sais pas, si elle seront acceptées ; d'après la réunion de ce matin, j'ai peu d'espoir, la procédure est lancée, c'est vraiment dommage $»^{21}$.

Au cours de cette seconde phase, un accord est trouvé : la réduction du temps de travail est échangée contre son aménagement. FO et la CFDT signent d'abord un pré-accord puis un accord. La position de la CFDT a été d'éviter les licenciements : «L'objectif majeur ayant dicté l'engagement CFDT a été d'éviter l'aboutissement du plan social ${ }^{22}$. Quant à la CGT, elle exprime sa déception, même si elle ne s'est pas opposée formellement à l'obtention de l'accord qu'elle ne signera pourtant pas : «Quand le fruit est pourri, on essaye de trouver la cerise et, là, c'est la cerise. C'est l'aspect positif qu'il faut voir : il n'y aura que 4 jours à venir au boulot. A se faire chier, on ne se fera chier que 4 jours au lieu de 5 ». Les positions divergentes des organisations syndicales traduisent ici l'ambiguïté de la position des salariés derrière les négociateurs : «J'ai eu l'impression que les gens

21 Extrait du procès-verbal de la réunion exceptionnelle du CE et des délégués des sections syndicales, CLARO, 1996. 22 Tract de la CFDT de mars 1996. 
râlaient avec la CGT et voulaient signer avec la CFDT. Il y avait de très fortes oppositions sur le samedi soir [...]. Il y avait une sorte de lassitude, il n'y avait pas une volonté d'affrontement [...]. Cela a été très dur de les faire se prononcer, ils ont voté à $90 \%$ quand même, en disant, nous, on est d'accord pour dire non au samedi soir avec vous, mais entendez vous avec la CFDT et FO pour faire des actions communes et proposer des choses ensemble », nous confie un responsable de la CGT.

\section{I.3. Travail du samedi soir et l'emploi}

Certes, cet accord n'est pas sans poser de problèmes pour les utilisateurs de l'accord, comme en témoigne les propos d'un salarié non syndiqué qui déclare : «Je vous dis bien qu'au niveau familial, le gars qui a des gosses qui font du sport, pour les suivre c'est perturbant. Comment voulez-vous qu'il fasse ? S'il faut y passer, s'il y a un malaise au niveau des licenciements, on ne va pas laisser tout le monde au chômage ».

Nous proposons d'interpréter maintenant les raisons pour lesquelles un tel accord a été obtenu et la signification de cet accord: (a) le temps de travail est un instrument de régulation de l'emploi et plus généralement de la régulation du marché du travail ; (b) l'emploi est un instrument de contrôle de la négociation puisqu'il a pour effet de modifier la position des acteurs comme les termes de l'échange ; enfin (c) le temps de travail a pour effet aussi de structurer le temps des activités professionnelles et des activités non professionnelles.

La première interprétation nous amènerait à conclure que le temps de travail est un instrument de régulation de l'emploi qu'il sert à maintenir. C'est ce qui semble se dégager et ce pour deux raisons qui se combinent : tout d'abord, il y a bien acceptation par les signataires d'une remise en cause des repères temporels antérieurs (travail du samedi, travail plus tôt le matin et plus tard le soir) en échange du maintien de l'emploi ; ensuite, il y a bien acceptation de l'augmentation de la flexibilité (modulation sur l'année des périodes de travail) et de la perte de salaire en échange de la réduction de la durée hebdomadaire.

La deuxième interprétation pourrait nous amener à considérer que l'emploi est un instrument de contrôle de la négociation sur le temps de travail. Cette interprétation se nourrit des faits suivants : rappelons qu'aucun accord n'avait été obtenu au cours des 15 dernières années sur le temps de travail et qu'il faut attendre 1996 pour que les partenaires sociaux trouvent un accord sur le travail du samedi. Mais cette négociation n'aboutit que lorsque la question de l'emploi intervient dans la discussion. Un événement semble avoir débloqué la situation : l'apparition début 1996 du plan social prévoyant la réduction des 
effectifs et entraînant, semble-t-il, la reprise des négociations et finalement la signature de l'accord: «Dans le projet de plan social, nous présentons, comme solution pour éviter les licenciements, de négocier à nouveau un accord d'aménagement et de réduction du temps de travail, $[\ldots]$ mais c'est la dernière chance » (représentant de la direction).

La troisième interprétation montre la relation entre un accord sur le temps de travail et ses conséquences sur l'organisation interne des activités professionnelles. La flexibilité ne touche pas que le cadre temporel, mais aussi l'organisation des diverses activités professionnelles. Tout laisse à penser, pour la CGT au moins, que le travail du samedi n'est que le symbole d'un changement de l'organisation du travail dans l'entreprise. Ainsi, les journées de travail sont maintenant de plus de $9 \mathrm{~h}$, ce qui amène beaucoup de salariés, résidant loin du lieu de travail, à se lever à 5 heures du matin pour aller travailler. Cette contrainte dérive directement ici de la concentration de la semaine de travail sur 4 jours. De plus, les équipes de travail semblent réorganisées, voire séparées, comme l'explique un responsable de la CGT : «Apparemment, ils cassent toutes les équipes. [...]. C'est quand même une organisation du travail qui est très rigide et donc les gens deviennent très rigides et c'est ça qui est un peu dur, parce que maintenant le patron dit : là, on va faire ça, il a dressé les gens en disant qu'il faut faire ça, tout est codifié, on travaille en millième d'heures, tout est hyper arrangé, planifié et tout». L'arrangement temporel sert-il d'instrument de contrôle sur le travail ? En tout cas, c'est la logique de la flexibilité qui semble l'emporter sur la réduction de la durée du travail dans ce « temps échangé ».

En résumé, la gestion de l'espace de négociation délimité par un contexte juridique oblige les négociateurs à prendre en compte l'articulation entre la flexibilité, l'emploi et la durée du travail. Ceci amène les protagonistes à changer de position, ce que nous illustrons par l'assouplissement de la position de la CGT face au durcissement de la position de l'employeur. Les termes de l'échange se ramènent à l'acceptation de la flexibilité contre le maintien de l'emploi. Ce n'est que lorsque le plan social a été au cœur des discussions que la position de la CGT a changé, ce qui est confirmé tant par la direction que par un responsable de cette organisation. Un responsable de la direction déclare : «En fin de négociation, la CGT a dit: on n'était pas en train de négocier un plan social, donc nous, nous disons non au travail du samedi. Si maintenant vous engagez une procédure qui menace effectivement 100 emplois, on peut discuter [...]. S'il y a plan social, on peut revoir notre position. Bien que le tract d'aujourd'hui ne semble pas manifester de la part de la CGT un revirement de position ». De même, le délégué syndical CGT déclare : « Nous, on a toujours dit qu'on se battrait contre le fait qu'il y avait des licenciements, mais on n'a jamais été confronté en fait tout au long de ces négociations à un réel plan social ». 
Des propos ci-dessus, nous en déduisons que la direction pensait que la CGT avait besoin du plan social pour faire passer sa « non-obstruction» à la signature de l'accord afin de ne pas être déjugée par ses adhérents. Nous en déduisons aussi que la CGT ne pouvait prendre au sérieux une menace de licenciements qu'à la condition qu'elle soit procéduralisée par un plan social. Le plan social et les licenciements deviennent donc paradoxalement pour un des acteurs syndicaux la condition de la non obstruction à l'arrangement temporel.

Observons que les termes de l'échange avaient bien changé : au triptyque des négociations antérieures basées sur les relations entre la durée du travail, la flexibilité et le salaire s'ajoute 1'emploi. Celui-ci joue un rôle de régulateur : «Au niveau des licenciements, si on peut en sauver quand même, il faut faire l'effort » (un salarié non syndiqué). L'emploi ne concerne pas directement la nature de l'arrangement temporel trouvé, mais bien la condition de son invention : c'est la raison pour laquelle nous pourrions interpréter, dans ce cas précis, le rôle de l'emploi comme un instrument de contrôle de la négociation sur le temps de travail.

\section{LE TEMPS DE TRAVAIL DES CADRES ET LES CADRES DU TEMPS DE TRAVAIL : LE CAS DE SINUS}

Le temps « individualisé » ne se limite pas à l'individu. Il renvoie à une pluralité de statuts d'emploi qui concerne à chaque fois des collectifs, c'est-à-dire des individus partageant les mêmes conditions de vie du point de vue du cadrage temporel des activités professionnelles. Dans le cas présent, le temps individualisé sous forme de temps partiel concerne les cadres d'une entreprise qui cherchent à échapper à la pression temporelle . Le souhait de mieux faire concorder les temps sociaux par d'autres arrangements temporels passe par l'individualisation du temps de travail d'une catégorie de salariés. Mais s'agit-il vraiment d'un groupe social distinct? Certains ont mis en avant une forte opposition entre cadres selon la fonction et le type de tâche exercé dans l'entreprise (Benguigui, Griset, Montjardet, 1978). Beaucoup pensent que c'est l'hétérogénéité même de ce groupe qui caractérise le mieux le groupe des cadres et le différencie des autres groupes, alors que cette hétérogénéité n'est pas propre aux cadres, (Groux, 1983, p. 9 ).

Dans notre cas, nous voudrions porter notre intérêt à ceux qui travaillent d'une manière ou d'une autre « au forfait ».

Ce groupe de personnes «au forfait» se distingue de celui du travail mesuré en temps. Néanmoins la notion du travail au forfait qui rappelle le travail à la « tâche », recouvre, elle aussi, des réalités différentes qui se caractérisent par un rapport au temps singulier des uns 
et des autres : les cadres les moins qualifiés préfèrent un contrôle strict de leurs horaires, alors que les cadres les plus qualifiés insistent sur une gestion plus souple de leurs horaires (Lallement \& Lefèvre, 1997). Dans notre entreprise, la hiérarchie des cadres traduit des comportements différentiels par rapport au temps : les plus bas placés demande l'instauration d'une pointeuse, alors que les cadres dirigeants ne veulent pas modifier leurs pratiques temporelles. Plus on s'élève dans la hiérarchie, plus l'autonomie temporelle est « chère » et considérée comme un avantage. Néanmoins, plus on s'élève dans la hiérarchie, plus les heures sont longues rappellent Bouffartigue et Boccino (1998, p. 43).

Ces auteurs posent plus généralement la question, si les cadres ne sont pas les précurseurs d'un temps incontrôlable dans l'entreprise : «Les ingénieurs et les cadres apparaissent largement piégés dans une problématique du temps de travail qui ne leur laisse guère le choix que de formes individuelles et privées de limitation ou de résistance aux pressions qui s'exercent sur eux », (op. cité, p. 49).

L'accord que nous allons présenter propose de sortir du «piège » par le passage à temps partielafin de limiter le temps des cadres. Ce temps partiel, sera-t-il capable de faire diminuer la pression des durées longues et irrégulières?

En 1996, un accord sur le temps partiel dans l'entreprise SINUS (secteur aéronautique) est signé par toutes les organisations syndicales. Pour certains des ingénieurs et des cadres qui représentent plus de $70 \%$ de l'effectif chez SINUS, ce temps partiel peut représenter une limitation individuelle de la durée du travail jugée « excessive », accord no 6. L'accord sur temps partiel permet alors de répondre « aux aspirations de certains salariés qui souhaitent concilier vie professionnelle et activités personnelles » (préambule, 1). Cette réduction de la durée du travail peut être individualisée un jour par semaine ou une semaine par mois. L'accord stipule le droit au travail à temps partiel pour les salariés, limité toutefois à $5 \%$ de l'effectif total des 1500 salariés, soit environ 75 salariés. Il permet notamment la possibilité de l'aménagement d'une semaine de 4 jours en admettant une perte salariale de $16 \%$. Comment est-on arrivé à une situation où les cadres se mettent à temps partiel ?

Auparavant, seule une trentaine de secrétaires, par accord individuel, travaillaient à $4 / 5 \mathrm{e}$ de temps. Le temps partiel possible par accords individuels, a été utilisé uniquement par les femmes (y compris des femmes cadres). En 1993, le premier des deux accords collectifs est signé et cette fois la majorité de ceux qui passent à temps partiel sont des hommes. 
Enfin, en 1996, un autre accord collectif sur le temps partiel est signé pour remplacer celui de 1993. Il prévoit du temps partiel à $50 \%$ (mi-temps), $60 \%$ (3/5e de temps), et $80 \%$ ( $4 / 5$ e de temps) et peut être déplacé de la semaine au mois ou à l'année ${ }^{23}$. Il a été signé par toutes les organisations syndicales dans un contexte de pression interne et externe sur la direction $^{24}$.

Auparavant, l'inspection du travail avait déclaré illégaux les contrats de travail des cadres sans mention d'heures précises, relançant ainsi la polémique sur les heures supplémentaires des cadres. Avec l'accord sur le temps partiel chez SINUS, le passage au temps partiel est devenu un droit et il peut être aménagé sur la base de journées, de demijournées ou de vacances scolaires. Des embauches compensatrices sont prévues.

«Toute personne qui dit: je veux passer à temps partiel, a le droit de passer à temps partiel, donc elle négocie les modalités avec son responsable d'équipe, il n'y a plus possibilité pour le management de dire : non, tu ne passeras pas pour telle et telle raison, c'est devenu un droit pour ces personnes-là, donc c'est une étape nouvelle sur le temps partiel » (responsable de la direction).

\section{II.1. A la recherche du temps de travail des cadres}

Chez SINUS, l'opposition entre deux formes de salariat (à l'heure et au forfait) différencie deux types de groupes sociaux, par leur temps de travail. Dans le premier cas, le personnel administratif, les ouvriers et les techniciens ont une durée de travail de $38 \mathrm{~h} 30$ par semaine ; dans le second cas qui concerne les ingénieurs et les cadres (70\% du personnel), la durée du travail n'est pas mesurée. Celle-ci s'élèverait, d'après une enquête ${ }^{25}$, à 45 heures en moyenne par semaine.

\section{II.1.A. Forfaitaires ou mensualisés : une distinction devenue obsolète}

Les cadres ont du mal à s'exprimer sur leur temps de travail. Ils ne peuvent pas le limiter individuellement par crainte des conséquences négatives sur leur vie professionnelle, comme l'indique un responsable de la $\mathrm{CGC}^{26}$ : « Beaucoup de gens se retranchent derrière, en disant : moi je ne peux rien faire de manière individuelle, sinon, je vais être le seul à le faire et je vais être dans le collimateur de mon responsable ».

23 Art. 4, accord 1996.

24 CFDT, CFTC, CGC et FO.

25 Etude du CE de SINUS, IPSOS-IST, 1996.

${ }^{26}$ Le sigle exact est CFE-CGC (Confédération française des employés-Confédération française des cadres) 
L'accord sur le temps partiel traduit un malaise de la part de ce groupe hétérogène à l'égard du temps de travail. Ce malaise porte sur un projet diffus et peu explicite, tourné vers la limitation de la durée du travail pour les cadres et les ingénieurs. En effet, la population des cadres est peu homogène : les niveaux hiérarchiques sont variables et seul $10 \%$ des forfaitaires font de l'encadrement. La grande majorité est composée d'ingénieurs de production ou d'experts techniques effectuant un travail qui peut se rapprocher de celui du technicien supérieur, comme le déclare un responsable de la CGC : «Le problème est qu'aujourd'hui, il y a beaucoup de personnes qui sont ingénieurs, mais ingénieurs vus comme des techniciens de très haut niveau. Ils maîtrisent pas mal de techniques et cette personne-là donc n'a pas une tâche fondamentalement différente d'un technicien, d'une personne dite mensuelle $\gg$.

L'industrialisation du secteur réduit les cycles de fabrication et réorganise le travail pour les cadres et les ingénieurs. L'évolution va du « savant fou » à l'ingénieur et au technicien de production. Les forfaitaires peuvent être amenés à travailler en équipe, la nuit et le week-end. Un responsable CFDT déclare : «Pour les forfaitaires, petit à petit, on sort d'une situation où on pouvait demander à un ingénieur et cadre de venir n'importe quand, sans qu'il y ait de notion de compensation. On a vu l'introduction de repos compensateur pour le travail du week-end, on a vu apparaître une notion d'indemnisation pour du travail pendant le week-end, chose qui n'existait pas avant. Donc les ingénieurs et cadres rentrent petit à petit dans un contrôle des horaires. [...] On va demander le pointage bien sûr, c'est ça le contrôle des horaires, c'est une mini-révolution culturelle».

Même si les cadres continuent à bénéficier d'une rémunération élevée (en moyenne $20000 \mathrm{~F}$ par mois contre $10000 \mathrm{~F}$ pour les mensualisés), les attentes à l'égard de leur travail pèsent (passage de l'artisanat à l'automatisation) et les menaces du chômage se sont faites ressentir lors du plan social en 1993. Les cadres chez SINUS, à l'exception des cadres aux fonctions hiérarchiques les plus élevées (10\% du personnel), représentent une catégorie de salariés dont la fonction d'encadrement s'amenuise sous le coup de l'industrialisation. C'est ce qu'un responsable CFDT déclare: «Après la guerre, un ingénieur avait une fonction d'encadrement et, comme on est toujours sous le régime des classifications, on a quelque chose qui ne correspond pas vraiment à la réalité ».

Une grande partie des cadres revendique à son tour, comme pour les mensualisés, le décompte des heures effectuées, s'il le faut par l'introduction du pointage. Ils demandent la transformation des heures supplémentaires en emplois et, pour eux, une réduction de la durée du travail. Le temps de travail des forfaitaires indiquait jadis l'expression de leur 
statut chez SINUS (« un travail intellectuel ne se décompte pas », responsable CGC) tandis qu'aujourd'hui le temps allongé représente un principe en retard sur lui-même.

\section{II.1.B. Le ras le bol des cadres face à la pression temporelle}

Que veulent les cadres par rapport au temps de travail ? Contre toute attente, une enquête effectuée (par IPSOS en 1996) chez SINUS à la demande du CE auprès de 493 personnes semblent révéler que $89 \%$ des cadres s'épanouissent dans leur travail. En revanche, ils sont aussi $90 \%$ à espérer que leur durée du travail diminue dans l'avenir, soit en adoptant la semaine des 4 jours ( $83 \%$ ), soit en passant à temps partiel (52\% de l'ensemble et $74 \%$ des employés). $70 \%$ de l'ensemble des salariés s'expriment pour le paiement des heures supplémentaires à partir de 40 heures. L'absence de contrôle des horaires des forfaitaires est d'autant plus rejetée que la position hiérarchique de ces forfaitaires est faible. En revanche, plus de $80 \%$ des employés mensualisés demandent le paiement des heures supplémentaires pour les forfaitaires.

Le niveau hiérarchique distingue les positions des cadres forfaitaires sur le paiement des heures supplémentaires : les moins bien positionnés demandent à $72 \%$ le paiement des heures supplémentaires (niveau I et II) tandis que les plus qualifiés (niveau III b et III c) ne sont que $48 \%$ à demander le paiement des heures supplémentaires.

La mesure du temps de travail pour les forfaitaires est soutenue par $70 \%$ des salariés puisqu'elle semble être l'étape obligatoire pour créer des emplois. $84 \%$ des salariés sont prêts à réduire leur temps de travail pour créer des emplois et $62 \%$ des salariés accepteraient une diminution proportionnelle de leur salaire en cas de réduction du temps de travail. On peut interpréter ces chiffres. Constatons d'abord que la grogne des cadres vient d'en bas: ce sont les catégories les moins bien placées dans la hiérarchie qui acceptent le moins les durées excessives du travail. Notons ensuite que «les durées excessives du travail» sont corrélées à une vie professionnelle plus difficile : $53 \%$ de l'ensemble des interrogés constatent une dégradation des conditions de travail depuis 1990, $55 \%$ mentionnent une hausse du stress et trois quarts d'entre eux constatent une hausse de la charge de travail $(74 \%)$.

\section{II.1.C. Réduction contre productivité : vers la semaine de quatre jours ?}

La direction semble prête, dans le contexte du travail en équipe comportant des cadres (accord 1995) ${ }^{27}$, à appliquer le même régime temporel aux forfaitaires et aux mensualisés, 
à condition d'augmenter la productivité du travail. Un responsable de la direction déclare : «On peut dire à des salariés : écoutez, si vous acceptez de travailler différemment, le matin, le soir, voire le samedi, voire le dimanche, vous allez de fait donner des possibilités nouvelles à SINUS par rapport à ses produits, par rapport à ses marchés et, en contrepartie, on peut aller effectivement vers la mesure de votre temps de travail ».

L'acceptation de nouvelles contraintes sur le travail des forfaitaires en échange d'une mesure de leur temps de travail a été concrétisée avec un accord sur le travail en équipes des cadres à titre expérimental concernant une centaine de salariés (1995).

Notons ici que, dans cette expérimentation, la mesure du temps de travail des cadres est conditionnée par l'augmentation de la productivité de leur travail. Néanmoins, SINUS n'est pas encore prêt pour la généralisation de cette expérimentation, comme le précise un responsable de la direction: «Je doute qu'on arrive à faire la même chose sur l'ensemble du procès parce qu'il y a des phases d'ingénierie dans lesquelles on peut pas mettre les gens en équipe. Toutes les activités ne se prêtent pas à ça [...]. Cela demande un travail de préparation, il faut la maturité des relais, des managers intermédiaires pour gérer tout ça positivement $»$.

Les organisations syndicales souhaitent néanmoins des solutions plus larges pour réduire le temps des cadres. Ainsi, un responsable de la CFDT qui représente l'organisation syndicale la plus importante (aux élections professionnelles) chez SINUS indique les termes de l'échange : «On est prêt à admettre une meilleure utilisation de l'outil industriel à condition qu'on passe à la semaine des 4 jours ». Mais, cette adhésion à un «temps échangé » et négocié (mesure et réduction du temps de travail contre davantage de flexibilité) n'est pas uniforme. Un responsable de la CFTC exprime au contraire un rejet de ce régime du travail en 4 jours, en particulier en raison du travail du dimanche : « La CFTC défend avant tout la vie de famille. Or aujourd'hui, il est clair que, s'il y a un des époux qui travaille le week-end et l'autre qui travaille n'importe quand, la flexibilité du travail va faire la flexibilité de la famille, et il y a suffisamment de famille monoparentale ».

\section{II.2. Le temps partiel : solution limitée pour cadrer le temps des cadres}

Après avoir présenté la nature de l'accord concernant le temps partiel des cadres, nous avons caractérisé les termes de la discussion : (a) une division obsolète basée sur un mode de comptage du temps de travail qui laissait de côté une partie des cadres (ceux au forfait),

« Il repose sur une modulation du temps de travail par cycle de 28 jours pouvant s'enchaîner sur l'année et sur la mise en place de 4 équipes successives » (Art. 4.1., accord de 1995). 
(b) le « ras-le-bol» des cadres devant dépasser le temps de travail légal sans obtenir de compensation, enfin (c) les termes de l'échange : du temps partiel contre une amélioration de la performance des cadres. Il nous reste à comprendre les enjeux de cet accord.

Nous avons dégagé trois enjeux : (a) des normes temporelles plus conformes aux pratiques des cadres et au contexte légal, (b) une carrière redéfinie, comme en témoigne l'histoire d'Adrien et enfin (c) le passage du travail au temps.

\section{II.2.A. Des normes temporelles plus conformes aux pratiques des cadres et au contexte légal.}

La direction n'a pas été favorable dès le départ pour signer cet accord, mais elle y voit aujourd'hui un moyen individuel de réduction du temps de travail pour les forfaitaires qui sont le plus opposé aux durées excessives du travail. Un responsable de la direction déclare : «Je suis convaincu que, dans les salariés qui vont passer à temps partiel dans les mois qui viennent, il y a des salariés qui estiment qu'ils passent trop de temps au travail et qu'ils ont là une façon officielle de réduire leur temps sur 4 jours. Ils continueront sans doute à travailler beaucoup sur les 4 jours, mais au moins ils voient concrètement qu'il y a un jour où ils ne travaillent pas. Donc, c'est vrai que cet accord sur le temps partiel va permettre de libérer un peu de pression côté salarié. [...] On pourrait se demander s'il n'y a pas une tactique de SINUS qui consiste à lâcher un petit peu sur le temps partiel en espérant que l'autre problème va tomber ».

Mais, il ne peut pas s'agir ici d'une stratégie à long terme, car la direction est bien consciente qu'elle ne peut résoudre le problème durablement de cette manière notamment en vue des réactions syndicales : «Ce n'est pas le fait d'avoir un accord temps partiel qui va dégonfler ou réduire le nouveau problème posé sur le temps des forfaitaires ».

Quant à ce changement de la position de la direction par rapport au temps partiel, la CFDT l'attribue à la situation interne et externe de SINUS. La pression de l'inspection du travail sur le temps non mesuré des forfaitaires a motivé la renégociation de l'accord et son extension à d'autres salariés. Un responsable de la CFDT déclare : "C'était assez remarquable d'ailleurs ce qui s'est passé parce que la négociation pour le temps partiel a commencé l'année dernière, au mois de juillet ou septembre, et ce que nous disait la société, c'est qu'elle n'entendait pas favoriser le temps partiel et elle nous l'a répété plusieurs fois. Il y a eu toute une série d'événements qui sont venus, qui ont eu à mon avis une certaine influence : il y a eu la lettre de l'inspection du travail qui a dit : le contrat de travail de $72 \%$ du personnel est illégal, il doit y avoir un contrôle de la durée du temps de 
travail [...]. L'accord temps partiel, ils ne voulaient pas le favoriser, et puis, si les organisations syndicales n'avaient pas voulu signer, il n'y en aurait pas eu. Du fait qu'il y a eu signature, ils ont même admis d'une certaine manière que ce soit un droit ».

La CFTC, par contre, critique cette voie du temps partiel pour réduire le temps de travail, car, selon ce syndicat, il s'agit d'un « luxe» d'inciter des forfaitaires bien payés à accepter une semaine de 4 jours. Un responsable CFTC déclare: «On a estimé que c'était inéquitable par rapport aux gens qui travaillent à temps plein, on a estimé que les gens qui travaillent à temps plein, pour eux, c'est une nécessité, dans la plupart des cas, et plus les salaires sont bas, plus c'est une nécessité, et donc ce n'était que des gens relativement aisés qui pouvaient s'offrir ce luxe, [...]. En plus, ça a été présenté sous forme de solidarité, alors qu'honnêtement, ça augmente le coût du travail, le coût horaire, et donc, une solidarité en augmentant le coût horaire, ce n'est pas très sain comme système ».

\section{II.2.B. Une carrière redéfinie : l'histoire d'Adrien}

Par rapport au problème des durées du travail, le temps partiel semble avoir un impact limité. Toutefois, son importance pour la vie au travail n'est pas à sous-estimer. Le passage à temps partiel continue à constituer pour les cadres un obstacle de fait puisque les mêmes raisons (pressions hiérarchiques) qui les obligent à des durées longues du travail, entraînent ici une vie professionnelle à temps partiel d'autant plus difficile.

Ce sont les conséquences pour la vie professionnelle qui empêchent l'acceptation du travail à temps partiel et non la perte de salaire. Un responsable de la direction déclare : «Ils ne sont pas à $5 \%$ de salaire près. Le problème est : comment vivent-ils leur vie professionnelle? Est-ce qu'ils font en quatre jours le travail qu'ils faisaient avant en 5 ? Est-ce qu'ils font, chez eux le cinquième jour, le travail qu'ils faisaient avant dans l'entreprise, en étant moins payés ? Il y a tous ces problèmes-là et je crois davantage qu'il peut y avoir du recul pour le passage à temps partiel plus à cause des problèmes dans l'entreprise que du salaire. Ce n'est pas le salaire qui les fera revenir à temps plein. Par contre, il y en a qui vivent, au niveau de leur équipe, une pression diffuse : tu n'étais pas là, ah, oui, toujours pareil, tu n'es jamais là le mercredi ; Ou bien : on a mis la réunion le mercredi, on n'avait pas le choix. Pour des gens qui vivent ça jour après jour, semaine après semaine, il y en a certainement qui vont dire : tant pis pour mon confort de vie, je repasse à temps plein parce que c'est invivable ».

Pourtant, malgré tout, certains forfaitaires «courageux» préfèrent de nouveaux arrangements entre vie professionnelle et vie hors entreprise au dépend de leur carrière 
professionnelle et de la progression de leur revenu, comme en témoigne l'histoire d'Adrien, responsable CFDT : «Je suis à temps partiel. Seulement, j'ai fait ça dans le cadre du plan social et je suis sur une base mensuelle. En fait, le principe en ce qui me concerne, est qu'on nous a demandé de choisir un jour dans la semaine ; moi, j'ai choisi le vendredi, et il était possible d'aménager ça sur une base, soit hebdomadaire, soit mensuelle, et quand vous choisissez ça sur une base mensuelle, vous êtes quand même obligé de choisir ; c'est la loi qui demande ça apparemment. Dans un mois comme le mois d'avril où il y a 4 vendredis, je regroupe mes 4 vendredis ce qui me fait une semaine de congé [...]. J'ai mes habitudes, j'arrive à peu près toujours à la même heure et je pars à peu près toujours à la même heure, le soir ; mais là, comme une semaine par mois, je ne suis pas là, c'est clair... je n'ai pas changé mes habitudes, je ne vais pas au-delà de 20 heures, j'ai effectivement réduit ma durée de travail, c'est clair. [...] La semaine où je ne suis pas là, sous certains aspects, c'est un petit peu embêtant, mais enfin dans l'équipe avec laquelle je travaille, on s'est arrangé entre nous, et donc, ça ne s'est pas trop mal passé. Au point de vue du plan de carrière, je ne suis pas le bon exemple puisque, de manière délibérée, j'ai choisi de ne pas avoir de responsabilités hiérarchiques. Par ailleurs, comme je suis considéré comme un expert au technique, je gagne relativement bien ma vie. Au niveau de l'évolution des salaires des gens, on s'aperçoit quand même qu'il y a un léger écart entre ceux qui sont à temps partiel et ceux qui sont à temps plein. Par heure, l'augmentation de la masse salariale des gens qui sont à temps partiel est inférieure à l'augmentation de la masse salariale des gens qui sont à temps plein, c'est 0,2\% d'écart, et c'est constant, c'est comme pour les femmes. De manière délibérée, il n'y a pas de volonté de payer moins les gens, mais dans les faits ça reste ».

Le temps partiel permet ici une limitation du temps de travail, soit sous forme de journées (les vendredis), soit de semaines entières. L'absence périodique du travail constitue le seul moyen efficace pour les cadres de se soustraire à la pression temporelle qui pèse sous formes de durées longues du travail. Le temps partiel constitue bien une soupape pour ceux qui ne veulent plus de ces durées, tout en admettant une vie professionnelle plus difficile sous le regard méfiant des équipes et de la hiérarchie. Cette stigmatisation du temps partiel chez SINUS montre bien que ce dispositif est considéré comme une forme minorée de travail à plein temps.

L'absence d'un engagement à plein temps pourrait être interprété comme un désengagement pour l'entreprise. Dans le cas du syndicaliste, il en découle une attitude de repli («pas au-delà de 20 heures, la carrière, ce n'est pas ma tasse de thé »), facilité par sont statut d'expert. Pour les collègues aussi, le temps partiel peut être contraignant, 
comme le déclare un responsable CFTC : « Il y a trois secrétaires. A un moment donné, il y en avait une qui travaillait à $4 / 5^{\mathrm{e}}$. Le vendredi, quand elle n'était pas là, c'est les 2 autres qui faisaient son travail, il n'y a jamais eu d'embauches pour 1/5e du temps ».

La direction ne peut pas tolérer que cet arrangement temporel soit appliqué à des ensembles plus vaste de cadres, car le temps partiel, et plus généralement un temps de travail sur mesure, constituent des freins à l'usage des capacités de travail.

\section{II.2.C. Du travail au temps}

En revanche, les syndicats semblent déterminés pour l'avenir à mesurer le temps de travail. Un responsable CFDT déclare : « ils vont résister \{la direction\} par rapport à ça parce que c'est toute une culture qui est remise en cause, quand même, et la culture, c'est la culture de la société qui veut que chacun se mobilise en fonction des événements, s'implique, etc., et qu'on ne compte pas son temps ».

Le pointage devient alors l'enjeu symbolique, non seulement de la mesure du temps de travail, mais aussi de la situation du groupe des forfaitaires. En effet, il s'agit d'un groupe hétérogène. Les managers et ceux qui ont de véritables fonctions d'encadrement s'opposent aux ingénieurs de production et aux experts techniques.

En revanche, du côté des ingénieurs, cette autonomie de gestion du temps est de plus en plus ressentie comme une contrainte de disponibilité qui va à l'encontre de leurs intérêts, en particulier lors de la réduction de l'emploi effectuée avec le dernier plan social. L'emploi, mais aussi le contenu du travail, amène la majorité des salariés à demander le paiement des heures supplémentaires et/ou à demander le passage à la semaine de 4 jours en temps partiel. De manière générale, l'objectif des organisations syndicales chez SINUS est de préserver la maîtrise de la durée du travail en utilisant des instruments suffisamment rigides (pointeuse, temps partiel sur 4 jours) pour cadrer les temps de la production du point de vue du personnel.

\section{LE TRAVAIL DE NUIT : FAUT-IL LE RESERVER AUX HOMMES? LE CAS DE FANA}

Jusqu'à une période récente, en France et dans d'autres pays européens, seuls les hommes étaient habilités à effectuer du travail de nuit dans l'industrie. Le changement des normes temporelles, conjugué à une pression de certaines femmes pour occuper des postes réservés jusqu'ici aux hommes, introduit un nouveau débat. L'objet est bien la légitimité du travail de nuit des femmes. Deux positions semblent s'opposer: d'une part celle qui vise à privilégier l'égalité des femmes préconisant la possibilité de travailler, comme les 
hommes, sur n'importe quel poste de nuit; d'autre part celle qui vise à protéger les femmes du travail de nuit.

Dans notre classification, nous avons rangé le travail de nuit du côté des dispositifs servant à étendre la durée d'utilisation des équipements : le temps des «machines » est celui de l'extension des plages horaires sur des périodes « traditionnellement » consacrées au repos. Le travail de nuit en constitue une forme particulière non seulement, parce qu'il comporte des risques graves pour la santé, mais aussi parce qu'il a fait objet jusque récemment d'une interdiction sélective pour les femmes. La négociation collective qui, depuis 1987, peut autoriser le travail de nuit des femmes. Un double accord (branche et entreprise) légitime alors le recours au travail de nuit féminin. Depuis 1991, cette situation est devenue plus complexe : un arrêt de la cour de justice de la Communauté Européenne donne raison à un employeur français, poursuivi en France pour cause de travail de nuit de ses salariées. Selon l'application d'une directive européenne (1976) sur l'égalité professionnelle des sexes, la cour déclare contraire à la législation européenne le principe de l'interdiction sélective du travail de nuit des femmes $(1892,1906)$. Conséquence de cette législation : aucun employeur en France qui fait travailler des femmes la nuit ne pourra être poursuivi pour cette raison à moins qu'il y ait une convention collective dans la branche.

La problématique hommes-machines se trouve dédoublé par le rapport de genres. De cette situation complexe Bué et Roux-Rossi (1993) font une analyse détaillée insistant sur l’hétérogénéité des positions syndicales.

Le travail de nuit des femmes y apparaît effectivement conçu comme un outil de la flexibilité. La négociation sur le travail de nuit des femmes et son instauration se fait toujours à la demande de l'employeur et le travail de nuit peut être présentée comme l'alternative aux licenciements féminins. D'après ces auteurs, les syndicats en entreprise, confrontés à des licenciements potentiels, sont dans une position difficile. Dans les cas analysés par Bué et Roux-Rossi (1993) les syndicats ne mettent jamais en cause l'extension du travail de nuit des femmes, à l'exception de la CGT. La négociation paraît très dissymétrique et le point de vue patronal s'impose très souvent. La décentralisation du niveau de la négociation, favorise une forte diversité des accords conclus. Bué et RouxRossi (1993) insistent aussi sur la notion du volontariat en montrant que les deux motivations principales pour l'acceptation du travail de nuit des femmes sont la menace du chômage et la rémunération plus élevée du travail. Cette question s'avère d'être un excellent révélateur de la division sexuée du travail professionnel. On peut se demander comment se gère la «mixité » des genres (Forté, Niss, Rebeuth, Trautmann, Triby, 1998) dans une situation où les femmes commencent à travailler la nuit avec les hommes. 
Comment les femmes qui représentent en journée la majorité des opérateurs de l'entreprise, peuvent-elles se faire une place au milieu de la nuit et parmi les hommes? Regardons le cas suivant.

En 1994, l'entreprise FANA du secteur de la métallurgie (2 100 salariés) signe avec FO un accord global sur l'aménagement du temps de travail qui touche à des domaines variés, comme par exemple le fonctionnement du travail en équipes et la rémunération.

Le présent accord a comme objectif :

«- de résorber le nombre estimé excessif d'opérateurs et d'opératrices en équipes de jour,

- de permettre au personnel féminin volontaire de travailler la nuit,

- d'améliorer la continuité de la production,

- de réduire le nombre de nuits travaillées de 5 à 4 par semaine,

- de répondre à la demande de travail à temps partiel,

- de diminuer le temps de présence des salariés » (article 1, accord 1994).

On pourrait penser à un accord classique qui réorganise le travail en accroissant la durée d'utilisation des équipements. Néanmoins, cet accord a la particularité d'avoir focalisé la négociation sur un point qui a priori concerne le travail de nuit des femmes et qui en constitue, du point de vue des acteurs, le dispositif central.

Ainsi, le dispositif central de cet accord concerne l'introduction du travail de nuit des femmes.

FANA, implantée depuis plus de 20 ans dans la région, connaît une croissance régulière de la production et de ses effectifs. La population salariale de FANA comporte un fort taux de femmes. Le nombre d'opératrices (450) dépasse celui des opérateurs (400). L'histoire du temps de travail chez FANA est caractérisée par deux faits majeurs. D'abord, nous avons relevé une difficile négociation sur la réduction de la durée du travail. En 1982, la réduction à 39 heures hebdomadaires n'est réalisée qu'après un mouvement de grève lancé par la CGT, la CFDT et FO. La direction et FO signent un accord sur un échange entre la réduction de la durée hebdomadaire et l'acceptation du travail de week-end (temps échangé). Ensuite, il faut souligner un impératif fort pour FANA de rentabiliser des installations coûteuses en faisant tourner l'usine 24 heures sur 24 et 7 jours sur 7 . Donc, le travail de nuit des hommes a toujours existé chez FANA. Enfin, en 1994, lors d'une extension de la production par de nouveaux investissements en machines, la direction propose un accord global sur l'aménagement du temps de travail centré sur le travail de nuit des femmes. 


\section{III.1. L'arrangement temporel est une construction sociale : le travail de nuit est négocié.}

Observons tout d'abord que cette entreprise s'inscrit dans un fonctionnement en continu sur toute l'année. Les caractéristiques de la production peuvent se résumer par trois éléments : installations coûteuses, salaires relativement élevés et travail par équipes successives. La production fonctionne en flux tendus.

L'accord a comme fonction d'organiser la continuité de la production ; l'aménagement du temps de travail est alors considéré comme une nécessité pour attirer de nouveaux investissements sur le site. Un responsable FO déclare : «On a fait des investissements pour que l'usine tourne 7 jours sur 7, 24 heures sur 24. C'est nous qui avons signé cet accord, sinon, les investissements qui ont été fait seraient partis ailleurs $»$.

Ces impératifs de la production se discutent et se négocient, car les acteurs de la négociation n'ont pas la même approche du temps de travail.

Les relations professionnelles chez FANA sont caractérisées par une opposition forte entre les syndicats ouvriers. D'un côté, il y a FO qui est majoritaire dans les institutions représentatives des salariés (plus de $50 \%$ de voix aux élections professionnelles), et de l'autre côté, il y la CGT et la CFDT. Un responsable de la CGT déclare : «La CGT et la CFDT y a au travail de nuit des femmes\} sont radicalement opposées et FO est radicalement pour, puisqu'ils ont signé ».

\section{III.1.A. Faire accepter le travail de nuit par le volontariat}

FO argumente sa signature par l'existence d'une liste d'attente de 17 femmes volontaires pour le travail de nuit : "A ce moment-là, il y a eu une forte demande féminine pour les équipes de nuit. Quand l'accord a été signé, les gens en parlaient depuis longtemps. Il y avait des personnes qui étaient intéressées, donc ils ont signé l'accord. Il y avait une liste d'attente ».

En revanche, la CGT conteste l'application du principe du volontariat pour le travail de nuit des femmes : «Une question qui revient à chaque fois, c'est le volontariat. Y compris en disant : mais vous ne signez pas l'accord alors qu'il y a des gens qui sont volontaires, des femmes qui sont volontaires pour travailler la nuit. Mais, dans les entreprises, tout au moins dans la nôtre, quand on connaît comment est fait le volontariat, ce n'est plus du volontariat.».

Pourtant, au moment de la signature, FO et la direction peuvent présenter une liste de 17 femmes pour le travail de nuit; un responsable de la CGT déclare : «Les fameuses 17 femmes, c'était pour avoir la signature. La direction a dit : ce sera de notre personnel ou 
des étudiantes, en l'occurrence du personnel volontaire. Or, tout ça, une fois l'accord signé, on fait n'importe quoi. Souvent, on leur en parle, vous avez signé ça, regardez, ce n'est pas une personne volontaire. Remettre son propre accord en cause, c'est difficile ». Du côté des syndicats minoritaires (CGT et CFDT), on observe des convergences au niveau de l'action syndicale. L'opposition avec FO s'exprime lors de la négociation de l'accord sur le travail de nuit des femmes. Du côté de FO, l'accord est ressenti comme un moindre mal et il apporte des satisfactions à l'ensemble du personnel.

\section{III.1.B. Le référendum pour infléchir la négociation}

En revanche, la CGT et la CFDT contestent la légitimité de l'accord et elles organisent, comme on l'avait fait chez «CLARO», une consultation chez FANA pour s'opposer au travail de nuit des femmes.

Peu après la signature de l'accord, la CGT et la CFDT procèdent à une consultation $\mathrm{du}$ personnel sur leur opinion face à l'accord. Les équipes de semaines qui sont les principales touchées par l'accord, répondent à 55,8\% au sondage mené par la CGT et la CFDT. $69 \%$ des personnes interrogées se prononcent contre l'accord sur le travail de nuit des femmes et $20 \%$ se prononcent pour cet accord. Parmi ceux et celles qui rejettent l'accord, $72 \%$ « veulent faire quelque chose » (tract syndical CGT).

Les formes d'action retenues par la CGT sont contestées par FO qui y voit un aveu de faiblesse de la politique syndicale de la CGT. Un responsable de FO indique : «Pour nous, faire un sondage qui n'est soumis à aucun contrôle, c'est nul. Une organisation doit avoir des gens dans toutes les équipes ».

Notons que cet accord sur le travail de nuit des femmes s'inscrit dans la pratique de l'accord de 1982. FO explique l'importance donnée à la notion du volontariat pour le travail du week-end. C'est la négociation de l'accord sur le travail de week-end en 1982 qui garantit aujourd'hui aux femmes de FANA le «libre choix» du travail la nuit en relation avec le travail du week-end. L'accord de 1982 sur le travail de week-end recouvre un arrangement tacite entre FO et la direction qui permet de contrôler l'application « juste» de l'accord de 1994. Un responsable FO déclare : «On aurait dénoncé l'accord \{de 1982\}, si la direction avait obligé des gens à travailler le week-end, mais ça ne s'est jamais passé. Ils sont 600 à y travailler. Notre démarche au moment de la négociation était d'obliger la direction à demander la démission des personnes qui étaient volontaires pour travailler le week-end, et ils signaient un nouveau contrat dans lequel ils gardaient leur ancienneté. Comme cette démarche d'obliger quelqu'un à démissionner est illégale, on a donc la possibilité de se retourner contre la direction ». 
La stratégie de FO visait alors, sur ce point précis, à mettre la direction dans l'illégalité pour le travail de week-end afin de contrôler si le volontariat pour le travail de week-end était respecté. Le cas échéant, il aurait suffi de s'adresser à l'inspecteur du travail pour faire annuler l'accord sur le travail de week-end.

Pour le travail de week-end, les signataires de l'accord mettent alors en avant l'inégalité des rapports de travail du point de vue du genre. L'impossibilité pour les femmes d'exercer le travail de week-end qui jusqu'ici est réservé aux hommes puisqu'il implique le travail de nuit, constitue alors une exclusion de fait.

\section{III.2. Des représentations sexuées du temps jalonnent la négociation de l'arrangement temporel}

«Pourquoi le travail de nuit des femmes? Parce que beaucoup de personnes voulaient passer le week-end. Or, il y a beaucoup d'horaires de l'équipe du week-end qui mordaient sur le travail de nuit, et les femmes n'avaient pas le droit de travailler la nuit, donc, elles ne pouvaient pas travailler le week-end ». C'est ainsi qu'un responsable FO qualifie la levée des restrictions faites aux femmes dans le domaine du travail de week-end. La codification du travail de week-end implique le travail de nuit. L'exclusion des femmes du travail de nuit provoque alors l'exclusion du travail du week-end qui constitue une formule avantageuse pour certaines salariées, car les 24 heures du week-end valent 39 heures en semaine. L'exclusion du travail de nuit constitue de ce point de vue un frein à l'embauche des femmes, mais aussi un frein aux choix des horaires de travail. Ces faits nous amènent à nous interroger en quoi les représentations des rapports de genres dans cette entreprise structurent le comportement des négociateurs ?

Si la direction met en avant la possibilité de l'embauche : «La direction avait demandé la signature de cet accord pour lui permettre de recruter des femmes techniciennes. Elle ne pouvait pas les recruter avant cet accord puisque les femmes ne pouvaient pas travailler la nuit» (responsable CGT), c'est bien pour assurer que la production tourne dans les meilleures conditions alors que le délégué FO argumente en « interne » pour l'égalité entre hommes et femmes chez FANA. Les questions de disponibilité et d'égalité sont ici difficiles à distinguer, mais nous tenterons de montrer que ce n'est ni l'impératif de la production, ni les bagarres syndicales qui donnent le sens de l'arrangement temporel, mais c'est bien d'une conception sexuée du temps chez FANA que l'arrangement tire sa légitimité ou au contraire s'en trouve rejeté. 


\section{III.2.A. La dévalorisation relative des qualifications de la main-d'œuvre féminine : progrès techniques et interdiction du travail de nuit}

L'embauche d'opératrices dans cette entreprise s'est effectuée pour les compétences particulières détenues par ces femmes. En effet, FANA a embauché massivement des femmes licenciées pendant les années 1980 et originaires du secteur du textile. Leur capacité à effectuer un travail manuel minutieux leur avait donné une préférence à l'embauche. Cette qualification spécifique initiale de ces femmes est aujourd'hui rattrapée par la rationalisation des techniques de production. Une opératrice déclare: "C'était valable, il y a quelques années, quand le travail était manuel. Maintenant non. Avant, il y avait beaucoup de couturières parce que le travail était minutieux, il demandait beaucoup de dextérité. Maintenant, ce n'est plus vrai. Avant, je me souviens, on a passé des tests pour être embauchées. Ces tests évaluaient notre dextérité, la coordination des mouvements, il fallait que l'on soit aussi rapide de la main droite que de la main gauche. On avait des postes manuels d'assemblage. Maintenant, ce n'est plus vrai ».

Quand cet avantage de la qualification des femmes disparait, l'exclusion du travail peut poser un problème. A partir du moment où la population de FANA est composée majoritairement par des femmes au niveau des opérateurs, l'interdiction du travail de nuit constitue un handicap pour certaines femmes, mais aussi pour la formation des hommes. Nous avons relevé l'exemple d'une opératrice qui dispense de la formation aux nouveaux salariés des équipes de nuit. Du fait de l'interdiction du travail de nuit, ces salariés de nuit ont été obligés de se faire former en journée par la personne qui est chargée de ces tâches. Or maintenant, après l'accord sur le travail de nuit, la concordance des temps professionnels semble mieux assuré. La formatrice explique : «Quand il n’y avait pas le travail de nuit des femmes, le personnel de nuit était obligé de venir le jour pour être formé. Ce n'était pas bien, ils étaient désorientés, ça ne leur convenait pas. Maintenant, c'est moi qui vais vers eux. J'aime les équipes de nuit $»$.

\section{III.2.B. La concordance des temps sociaux au cour des représentations sexuées du temps}

En tout cas, le travail de nuit semble être accepté par les femmes qui se trouvent sur un poste de nuit. Les équipes de nuit disposent d'une marge de manœuvre plus importante puisque le contrôle hiérarchique est moins présent. Une opératrice de nuit explique : «Que ce soit de jour ou de nuit, un opérateur ou une opératrice qui ont le même poste, ont exactement le même travail. Il n'y a aucune différence. La nuit, le travail est différent en ce sens qu'il y a moins de contraintes. Dans les secteurs de jour, il y a beaucoup de 
sollicitations dues au service. Par exemple, il est fréquent qu'un service ait besoin d'un ingénieur. La nuit, ils ne viennent pas. Un opérateur a 5 machines à surveiller. La nuit, il les a pour lui tout seul. Le jour, il a aussi 5 machines, mais il doit parfois en prêter une à un ingénieur. Mais l'opérateur devra également aider l'ingénieur, car celui-ci ne connaît pas tout du fonctionnement de la machine. Il doit donc arrêter son travail. La nuit, si une machine marche bien, personne ne vient le gêner ».

Mais ceci ne constitue pas la seule raison de vouloir travailler la nuit. Une des raisons ayant poussé certaines femmes à demander le travail de nuit concerne la possibilité de faire garder les enfants la nuit, de pouvoir s'en occuper dans la journée en dehors de l'école, et aussi une rémunération nettement plus élevée. Une opératrice déclare : "Pour une partie, c'était beaucoup à cause des problèmes de garde des enfants, les petits ou ceux qui allaient à l'école. Donc, les mères peuvent prendre des nourrices ou des jeunes filles au pair pour garder leurs enfants la nuit. Comme ça, le lendemain, elles peuvent les préparer, les emmener à l'école. Après, elles peuvent se reposer jusqu'à midi et faire à manger à leurs enfants, s'ils rentrent. Le soir, elles peuvent s'occuper de leurs devoirs. Et en plus, il y a une prime d'équipe. Elle est passée de 6 à $30 \%$, ce qui n'est pas négligeable ».

En revanche, d'autres femmes semblent avoir refusé le travail de nuit en indiquant l'incompatibilité entre travail professionnel, travail domestique et vie familiale. Un responsable de la CGT explique : «La catégorie des femmes mariées ou avec charge de famille et des enfants a refusé majoritairement parce que ce n'est pas conciliable. Déjà que les journées des femmes sont longues à mon avis, alors là, ça fait des journées pas possibles. Quand on a des enfants, on arrive à 7 heures et demie le matin à la maison en moyenne et il faut emmener les enfants à l'école, il faut retourner les chercher, s'en occuper le soir. [...] Les divorces grimpent en flèche, c'est flagrant. Sur les 22 femmes qui ont accepté de travailler la nuit depuis 3 ans, il y en avait 15 ou 16 qui étaient mariées, il n'en reste que 2 ».

Les arguments dans ces deux propos, bien qu'opposés sur le travail de nuit, se rattachent systématiquement à une vision sexuée du temps. La gestion de la famille et des tâches domestiques relève dans la réalité et dans les représentations de la responsabilité des femmes. Il faut assumer cette responsabilité, peu importe si on travaille la nuit ou le jour, et les hommes semblent dans les discours exclus de ces tâches. Ainsi, si la plupart des couples ont divorcé suite au travail de nuit des femmes, c'est parce que la compatibilité des temps du couple relève aussi de la responsabilité des femmes, peu importe, si le mari travaille déjà la nuit ou le jour. L'obligation implicite de la prise en charge de l'univers 
domestique par les femmes n'est mise en question ni par les partisans, ni par les adversaires du travail de nuit des femmes.

\section{III.2.C. Le travail de nuit des femmes : un « seuil de tolérance » pour maintenir les femmes à l'écart ?}

Plus généralement, FANA s'est posée la question de la mixité des équipes de nuit qui étaient jusqu'ici réservées aux hommes. Le nombre de femmes qui se retrouve dans une équipe d'hommes, a introduit chez certains une réflexion en termes de " seuil de mixité » qui démontre que l'égalité hommes-femmes au travail peut se heurter à des représentations des salariés sur un taux de femmes «acceptable » pour le travail de nuit. Une opératrice déclare : «Il y a beaucoup d'ingénieurs qui sont rentrés chez FANA, ce qui a fait diminuer l'effectif des femmes. En équipe de nuit, il y a au plus 20-25\% de femmes. L'effectif augmente en fonction du nombre de postes qui s'ouvrent. Mais si les postes de nuit augmentent, ils seront complétés indifféremment par des hommes ou par des femmes. Personnellement, je pense que c'est une bonne proportion de femmes qu'il y a la nuit, 20$25 \%$. S'il y en avait plus, il y aurait des problèmes, notamment au niveau relationnel ».

Notre hypothèse interprétative est que l'argumentation sur les prétendues difficultés de la mixité des équipes de travail correspond à la volonté des hommes de maintenir le contrôle de la gestion du marché interne de l'emploi et de tenir à distance de certains emplois et de certains avantages consécutifs à l'occupation de ces emplois les femmes : il y a donc une lutte autour de l'hégémonie du référentiel masculin de gestion.

Au niveau relationnel, le travail de nuit génère apparemment des meilleures relations entre hommes et femmes au travail. Une opératrice déclare: «Dans mon équipe, il y a 17 personnes dont 4 femmes. Pendant la pause, le personnel est partagé en deux. La moitié part en pause et l'autre s'occupe des machines, et inversement. Même quand il y a une dispute entre les femmes, ce n'est pas la même chose de jour que de nuit. Les disputes du jour ne se voient pas la nuit. En jour, elles durent parfois une semaine, la nuit, elles règlent ça d'homme à homme. Elles en parlent 5 minutes et après, c'est fini. [...] Elles ont été bien accueillies par le personnel masculin. Maintenant, ils ont l'habitude. Il n'y a pas de problèmes entre les hommes et les femmes. Il peut très bien y avoir des problèmes, mais ce n'est pas parce que les femmes travaillent la nuit. Même au niveau du langage, ça se passe très bien. Même quand il y a une fille de bien, les garçons ne font pas de réflexion. Il y a de la retenue. Ca se passe très bien. [...] On peut très bien parler de voiture, comme des enfants, de chiffons, de cuisine, qu'il y ait des garçons ou non. On aborde tous les sujets. Il 
n’y a pas de séparation dans les discours. Dans chaque secteur, les femmes ne sont pas assez nombreuses pour pouvoir former un groupe ».

L'état de minorité numérique des femmes dans les équipes de nuits les fait changer d'univers de travail. Alors qu'elle peuvent se retrouver pendant la journée en majorité, elles ne sont guère plus de 5 à travailler dans une équipe de nuit de 20 personnes. En cas de divergences entre les femmes la nuit, « elles règlent ça d'homme à homme ». L'expression de l'opératrice indique bien l'existence d'un univers de travail qui incarne particulièrement bien la norme du travailleur industriel masculin. L'interdiction du travail de nuit des femmes pourrait être alors interprétée comme une protection de ce modèle. En effet, l'assimilation des femmes ne s'est pas faite sans résistances, en particulier du côté de la maîtrise. Il semble que certaines équipes, et notamment certains agents de maîtrise, aient eu des craintes quant au fonctionnement de la mixité hommes-femmes. Une opératrice indique : «Pour certains agents de maîtrise de nuit, ils appréhendaient les histoires que les femmes pouvaient faire. C'était une équipe d'hommes très soudée. Et ils ont donc eu peur que l'introduction des femmes crée des tensions au sein de leur équipe. Elles ont été intégrées petit à petit. Dans un secteur, il y avait une ou deux femmes dans un groupe d'hommes, il n'y a pas eu de secteur où il n'y avait que des femmes. Elles sont arrivées petit à petit au milieu des hommes. Maintenant, il n'y a plus de problème. Cela se passe très bien. Par contre, s'il y avait eu au début 30 femmes dans un groupe d'hommes, ça n'aurait pas marché. Il fallait qu'elles s'assimilent ».

Les discours sur l'assimilation traduisent pour nous ici des représentations visant à normaliser les comportements professionnels des femmes sur le modèle des hommes. Les femmes arrivent en territoire « ennemi» et doivent se faire une place dans «l'équipe soudée » des hommes. La hiérarchie intermédiaire semble particulièrement sensible aux menaces que les femmes constituent à l'égard du modèle dominant. Rappelons que les femmes chez FANA sont essentiellement des opératrices en bas de l'échelle hiérarchique.

Un responsable de FO déclare : «En maîtrise, ils ne voulaient pas embaucher de femmes parce qu'ils avaient peur des histoires $»$.

La peur des « histoires » signifie peut-être au fond la peur de perdre des positions acquises. Celles-ci sont sans aucun doute favorables aux hommes. Les différences de salaires indiquent une discrimination des femmes au travail. Le bilan social de 1996 de l'entreprise nous montre un salaire quasi-identique pour les opérateurs et les opératrices, mais plus on s'élève dans la hiérarchie, plus l'écart se creuse. Ainsi le salaire moyen d'un homme-cadre dépasse de $30 \%$ le salaire d'une femmes-cadre. Le temps de travail chez FANA et la disponibilité des salariés par rapport aux demandes de la direction semblent servir 
d'argumentation pour maintenir l'inégalité hommes-femmes au travail. Un responsable FO déclare : «Pour les opérateurs, il y a égalité, par contre, chez les cadres, les hommes gagnent plus que les femmes. Mais c'est à cause de la disponibilité. Cela ne dérange pas un homme de partir 3 mois aux Etats-Unis ou 2 mois en Allemagne, alors que les femmes ne peuvent pas, à cause des enfants. Elles sont moins disponibles que les hommes, donc moins payées, puisqu'elles travaillent moins ».

Donc, malgré l'introduction du travail de nuit pour les femmes, de fortes inégalités subsistent, comme l'indique un responsable CGT : «Alors que cet accord est en place depuis 3 ans, on s'aperçoit que la discrimination entre les hommes et les femmes est de plus en plus importante. Quand on sait que le taux de femmes dans les écoles d'ingénieurs oscille entre 23 et $27 \%$ de l'effectif, ici, on atteint à peine $8 \%$ des cadres qui sont femmes et encore, on arrive à 2 ou $3 \%$ dans les catégories les plus élevées ».

L’impact limité de l'accord (seules 25 femmes travaillent la nuit) ne peut pas dissimuler sa valeur symbolique puisqu'il pose, à travers le temps, la question de l'égalité entre hommes et femmes à divers niveaux (salaire, qualification, statut, rapport hommes-femmes au travail, rapport hommes-femmes dans le domaine familial et domestique). Il fait ressortir le référentiel négociatoire des syndicats et les principes structurants de l'action syndicale. Ainsi, la CGT qui est fortement opposée au travail de nuit des femmes, explique sa position: «C'est l'inégalité entre les hommes et les femmes de dire que les femmes ne sont pas égales parce qu'elles ne peuvent pas travailler la nuit. Alors nous on dit, au contraire, il faut que tout le monde travaille le jour. La nuit, c'est fait pour dormir ».

Cette position coïncide (selon la CGT) avec le souhait des salariés de disposer d'un cadre temporel régulier : «Le personnel veut quand même, dans sa grande majorité, un rythme de travail régulier. C'est pour cela que l'on s'aperçoit que les horaires quand même les plus contraignants sont ceux qui sont le moins facilement acceptés. Par exemple, les trois huit. Il y a très peu de personnes qui sont en trois huit parce que c'est un horaire qui est très contraignant, une semaine le matin, une semaine l'après-midi, une semaine la nuit. Je pense qu'ils connaissent les limites à ne pas franchir, les limites que le personnel n'accepterait pas ».

La CGT met alors en avant la primauté des temps des hommes et des femmes par rapport au temps des machines : «Nous, on dit : plutôt que de mettre une personne le matin, une personne l'après-midi, une personne la nuit pour fabriquer un produit, on peut mettre 3 machines. Donc, la personne travaille en journée normale, et avec 3 machines, on fait le même produit mais là, la rentabilité n'est pas la même ». 
Sous forme de conclusion, nous pouvons proposer quelques hypothèses interprétatives. D'abord, nous pouvons constater que l'exclusion des femmes du travail de nuit et, par là du travail de week-end, ne semble pas résister aux impératifs de disponibilité et du temps des machines. La question de l'égalité des sexes apparaît dans le contexte de l'intersection de projets différents sur le temps de travail, exprimés par les syndicats et la direction. Le syndicat signataire se trouve ici du côté de la direction pour défendre le droit des femmes au travail de nuit et de week-end. Les syndicats opposants dénoncent l'accord en critiquant la primauté du temps des machines sur celui des hommes et des femmes. Les acteurs cherchent à légitimer leur démarche auprès des salariés. FO et la direction avancent la liste des volontaires pour le travail de nuit tandis que la CGT et la CFDT s'appuient sur un référendum pour montrer l'opposition globale des salariés. En revanche, l'accord n'a pas contribué à faire disparaître la division entre hommes et femmes chez FANA, ni les représentations sexuées du temps de travail comme le montre le problème de la mixité des équipes de nuit. Les différences de salaires se creusent avec le statut de l'emploi et, au niveau des techniciens et des ingénieurs, les femmes n'occupent que $20 \%$ des postes alors qu'au niveau de l'exécution, elle représentent plus que la moitié de l'effectif (bilan social 1995). Les obligations implicites du travail domestique et la demande explicite de mobilité et de disponibilité pénalisent les femmes dans l'exercice de leur activité professionnelle. On peut faire l'hypothèse que l'encadrement intermédiaire (de terrain) met en relief la crainte (dans certains cas) que le modèle du travailleur masculin évolue sous la pression de la présence des femmes. Ainsi, on peut expliquer l'existence d'un raisonnement en termes de «seuil de tolérance » dans cette entreprise quant à la participation des femmes dans les équipes de nuit.

\section{L'ANNUALISATION ET L'INCOMPATIBILITE DES HORIZONS TEMPORELS REELS : LE CAS DE ROTOR}

Le temps des «marchés » est celui qui calque les temps de travail des employés sur les mouvements des marchés de clients, en faisant varier la durée hebdomadaire sur une plus longue période. Dans le cas présent, il s'agit de l'année. Caissières et autres employés de cette entreprise de supermarché se voient proposer dans un accords d'entreprise l'annualisation de leur temps de travail. Que toutes les caissières soient à temps partiel et tous les autres employés à plein temps ne constitue pas la seule particularité de cette situation. Nous verrons que la conception de cette annualisation déplace sur l'année la différence entre temps plein et temps partiel en semaine. En revanche, ce simple 
déplacement du mode de calcul du temps de travail qui ressemble à un exercice mathématique, s'avère incompatible avec le fonctionnement concret de l'entreprise au jour le jour. L'entreprise qui vit largement sur la disponibilité de ses employés ne peut pas planifier les temps annuels et doit donc abandonner l'idée d'une annualisation du temps de travail. Comment une telle solution temporelle approuvée auparavant par la direction et le représentant syndical peut-elle échouer sur le terrain?

La question de l'annualisation du temps de travail déplace le calcul du temps de travail de la semaine à l'année. Une forme particulière d'annualisation, inaugurée fin 1993, concerne le temps partiel annualisé permettant pour les salariés à temps partiel une répartition inégale, mais prévisible du temps de travail sur l'année. Un accord sur cette question a été conclu dans l'entreprise ROTOR du secteur du commerce de détail en 1995 (92 salariés et une vingtaine de petits magasins). Pour les petits magasins de vente d'alimentation, l'accord porte sur «la mise en place d'horaires annualisés pour le personnel» (titre de l'accord). Le préambule de cet accord chez ROTOR indique que «l'activité prévisionnelle de ces nouveaux magasins est difficile à appréhender, la fréquentation de la clientèle y fluctue selon les semaines et les saisons et, de plus, ils présentent une grande vulnérabilité face à la concurrence » (accord 1995, p. 2). L'accord définit des durées annuelles pour les caissières (1150 heures) et pour les employés principaux (1472 heures). L'amplitude de la modulation sur l'année peut varier, dans le respect des dispositions du code du travail, entre 10 et 39 heures sur l'année pour le travail à temps partiel. En revanche, et contre toute attente, comme nous le verrons plus loin, cet accord signé entre la direction et FO n'a jamais été appliqué chez ROTOR.

\section{IV.1. Toutes les caissières sont à temps partiel}

Cette entreprise de commerce, installée depuis quelques années seulement dans la région, doit faire face, d'une part, aux fluctuations des effectifs dans les magasins à cause des absences imprévisibles des employés et, d'autre part, aux fluctuations de l'arrivée des marchandises. Cette mobilité du personnel est obtenue avec des contrats à temps partiel. En effet, la structure temporelle est basée sur un référentiel hebdomadaire et sur du temps partiel pour les caissières ( 25 heures) et pour les employés principaux ( 32 heures). Comme l'explique le seul responsable syndical de ROTOR, l'embauche des caissières à temps partiel est plus rentable : «La direction veut que les caissières fassent 25 heures, tout simplement à cause de tout ce qui est charge sociale, donc ils veulent bien faire faire des heures supplémentaires, si c'est nécessaire, même si au total on s'aperçoit que sur la 
semaine par exemple, le nombre d'heures effectuées en supplément représente le travail d'une autre personne : à la limite, ça va leur coûter moins cher en heures supplémentaires que d'embaucher quelqu'un ».

Dans la vie quotidienne de ces salariés, une cascade d'avenants au contrat de travail intervient pour légaliser les pratiques des recours aux heures complémentaires. Le système de vente se retrouve alors déstabilisé par des cas d'absences imprévues que ROTOR ne peut gérer qu'en transférant, dans des délais réduits, du personnel d'un site à l'autre. Cette situation est alors vécue comme contraignante pour la direction qui doit occuper une partie de ses cadres à la gestion juridico-économique de ces problèmes. Or, le responsable syndical regrette cet abus du recours aux heures complémentaires. Il déclare : « en plus, c'est illégal parce qu'au bout d'un certain nombre d'avenants, normalement, la personne doit passer au nombre d'heures défini. Donc les filles, au lieu de faire 25 heures, elles font 32 heures depuis 1 an, toujours avec des avenants. Donc un jour, parce que ça va m'énerver, je vais demander à l'inspecteur du travail de passer, et puis les filles, elles seront passées à 32 heures.».

Ce recours systématique aux heures complémentaires assure les besoins de disponibilité de ROTOR, mais il est vécu comme contraignant par les salariés qui peuvent être envoyés dans des délais très courts, à des endroits éloignés de leur lieu d'habitation (100 km). Une responsable de la direction ajoute : " avec les caissières, les chefs de magasins, ça fait quand même peu de monde, et surtout c'est très éclaté, le problème est qu'on a un éclatement géographique très important $»$.

A l'inconvénient de gérer des toutes petites structures de vente (5 salariés), se rajoute une recherche constante de la part de la direction de tourner avec le moins de personnel possible.

En effet, la direction donne des objectifs de réduction de coûts difficiles à tenir pour les petits magasins : " la direction a des objectifs de frais de personnel qui sont réduits au maximum, et c'est à nous de nous débrouiller avec ça ».

Notons que cette situation se traduit aussi pour les employés principaux (chef de magasin) par des durées du travail très longues. Payés à un forfait temps plein sur 42 heures, ces salariés peuvent travailler jusqu'à 60 heures par semaine sans paiement des heures supplémentaires, comme l'indique le responsable syndical, lui même chef de magasin : «C'est nous qui nous prenons les heures supplémentaires qui ne sont pas payées. Donc, ça ne me dérange pas de faire des heures supplémentaires, c'est pas le problème, mais quand on fait 60 heures toutes les semaines payées 42 , il y a un moment où on commence à en avoir ras le bol ». La situation chez ROTOR présente bien deux populations de salariés : 
d'un côté, les personnes embauchées à temps partiel de 25 heures (caissières) avec une utilisation très importante des heures supplémentaires et un fort impératif de mobilité d'un site à l'autre géré par des avenants au contrat de travail, et, de l'autre côté, les plein-temps auxquels on demande une grande quantité d'heures supplémentaires non-payées. Comment arranger la situation du personnel sans embauches supplémentaires ?

Pour remédier à cette situation, le délégué syndical et la direction trouvent un terrain d'entente autour de la conclusion d'un accord sur le temps partiel annualisé.

\section{IV.2. L'annualisation du temps partiel : une sortie du dilemme de la gestion des durées du travail?}

\section{IV.2.A. Négocier le temps partiel annualisé avec un syndicalisme réduit}

Chez ROTOR, une seule personne représente dans des conditions difficiles les 92 salariés éclatés en 20 magasins : «je cumule trois mandats : j’ai le CE, les délégués du personnel, et le délégué syndical, donc j'ai 20 heures pour le CE, j'ai 15 heures pour les délégués du personnel, et 10 heures pour le délégué syndical. Ce qui fait que j'ai 45 heures par mois, que je ne peux pas encore prendre pour l'instant entièrement, vu notre personnel réduit ».

En effet, en tant que chef de magasin de la première heure, il a pu connaître l'ensemble du personnel et c'est la direction qui lui demande s'il veut faire le travail du délégué syndical.

Le responsable syndical (FO), signataire de l'accord, décrit la situation de la négociation : « c'est le responsable qui est venu me voir, en disant : voilà, on voudrait modifier le temps partiel, et on voudrait donc plutôt que de faire des contrats de 25 heures, faire un contrat à l'année sur un nombre d'heures dans l'année. On accorde un crédit d'heures à la caissière, de tant d'heures par an, donc c'est le chef de magasin qui va gérer ce nombre d'heures, et qui décidera. La caissière, tous les mois, elle aura la même paye. Donc pour elle, ça n'aura aucune incidence de faire 8 heures une semaine ou 32 l'autre : c'est vous qui gérez votre planning en fonction des capacités ou du travail qu'il y a à faire à tel ou tel moment ».

Sur cette proposition de la direction, le syndicaliste a tout de suite compris que pour les caissières une telle gestion pouvait avoir des avantages en termes de prévisibilité de leur emploi du temps, devenue impossible pour des raisons liées à la gestion des temps partiels à court terme, mais aussi du fait d'une cascade d'avenants. 


\section{IV.2.B. Codification annuelle du temps à risques : une coalition tacite pour ne pas appliquer la règle.}

Les espoirs projetés sur l'accord ne se sont pas concrétisés. Il n'a jamais été appliqué chez ROTOR. Nous constatons effectivement que quatre facteurs font de l'accord une règle non appliquée.

Constatons d'abord une flexibilité limitée par l'insuffisance de la main d'œuvre disponible au regard du travail à faire. Si l'accord est apprécié par les caissières, la crainte de perdre même temporairement des heures de travail pour suffire au repos est toujours bien présente. Le responsable syndical déclare : «j'en avais parlé à quelques personnes que j'ai eu le temps d'avoir, puis la plupart m'ont répondu à peu près la même chose, oui, $\mathrm{OK}$, c'est vrai que c'est bien, mais, bon, on est 3 dans le magasin, on est déjà trop serré, je peux pas me permettre d'enlever 1 personne, ou de la faire travailler moins ». Le processus de travail est déjà largement rationalisé et, de ce fait, il laisse peu de réserves de main d'œuvre disponibles.

Ensuite, nos interlocuteurs (direction et délégué de FO) nous attestent qu'il existe une discordance entre l'exigence de réactivité au jour le jour (court terme, horizon quotidien) d'un simple temps partiel et l'exigence réglementaire de prévenance pour le calcul des temps annualisés (prévision à moyen terme sur un horizon d'une semaine). Pour une responsable de la direction, «du fait qu'on est éloigné de tous nos sites, on est obligé d'avoir des superviseurs qui vont dans les magasins, qui traitent de tous les problèmes. Et dans les superviseurs, on a comme partout des bons et des mauvais et on a régulièrement des problèmes avec le personnel quand un superviseur qui est passé dans un magasin n'assure pas du tout sa fonction de personnel ».

De plus, l'accord est interprété comme un « écran » entre la direction locale et ses salariés. D'un côté, l'accord repose sur une codification des temps sur un horizon annuel et, de l'autre, l'accord rend possible des calculs stratégiques d'épargne-temps dans le cadre des arrangements locaux négociés avec la hiérarchie de proximité. Caissières et employés principaux mettent en place des solutions locales avec les chefs de magasins. Mais, ces solutions locales, adaptées aux contextes et établies dans un cadre réglementaire, constituent des arrangements explicites. Elles sont variables d'un magasin à l'autre et ne peuvent pas être décrétées par une gestion centralisée. Par voie de conséquence, la direction ne peut exercer d'en haut la définition de ces arrangements. La direction régionale exprime un manque de confiance sur la gestion directe des heures par la hiérarchie intermédiaire. La gestion de la flexibilité sur l'année renforcerait, selon une responsable de la direction, la position de cette hiérarchie intermédiaire et la direction 
craint que l'état réel des heures travaillées soit dissimulé sur une longue durée par des pratiques locales de gestion qu'elle ne contrôle pas.

Enfin, une dernière contrainte va dissuader les acteurs d'appliquer l'accord : l'annualisation des temps partiels génère du « crédit bloquant ».

L'accumulation d'heures complémentaires par des salariés dans certains magasins pourrait mener à une situation où, après 9 mois de l'année, le salarié aurait rempli son contingent annuel. Pendant les 3 mois restant, le salarié sera en congé et le magasin sera alors privé des ressources nécessaires pour son fonctionnement et il sera obligé d'embaucher des remplaçants. La responsable de la direction explique sa crainte de l'application de l'accord : « un jour, on va se retrouver avec 9 mois où on croit que la personne a encore plein d'heures à faire. Ce qui leur importe, c'est leur paye, donc là on va se retrouver dans des situations complètement faussées et pas possibles. »

Le délégué syndical reconnaît cette difficulté à la direction, il rajoute : « il faut que ça soit bien suivi au niveau du chef de magasin, quand il fait des heures supplémentaires, qu'il puisse le justifier, il n'y aura aucun problème par la suite pour faire des heures complémentaires. S'il fait faire des heures parce que lui, il a envie de se la couler douce dans le bureau, forcément, c'est ensuite à la direction de s'apercevoir de ce qui se passe dans le magasin »

Soulignons ici la contradiction fondamentale entre, d'un côté, les acteurs qui se mettent d'accord sur une codification du temps de travail sur une base annuelle et, de l'autre côté, le fait que cette solution ne puisse être appliquée précisément parce que l'annualisation va à l'encontre des réalités locales des horizons temporels des magasins.

D'abord, nous retenons que la signature de l'accord est une solution acceptable pour les parties en présence.

L'annualisation constitue un horizon d'acceptation du temps de travail, un outil de comptage et de sommations des durées effectivement travaillées, une référence normative à ne pas dépasser. C'est donc un mode de structure temporelle des espaces professionnels, dans laquelle deux logiques d'actions sont en présence : côté salarié, l'acceptation d'une variabilité des durées hebdomadaires en échange d'une possibilité de calcul et de gestion stratégique de son enveloppe temporelle sur l'année; côté employeur, le respect d'une norme annuelle (1150 ou 1472 heures) en échange d'une possibilité de régulation temporelle à court terme (sur l'horizon de la semaine) calquée sur le temps des marchés.

En revanche, l'absence de l'application résulte d'une codification à risque. On pourrait émettre l'hypothèse que la non-application est un accord tacite qui arrange les parties en 
présence du fait de l'incompatibilité potentielle entre la logique de la semaine sur l'horizon annuel et la logique de la régulation sur l'horizon hebdomadaire. Cette incertitude du résultat concerne la correspondance entre une norme annuelle et la sommation des durées hebdomadaires variables, et conduit les acteurs à préférer la non-application de l'accord. Si l'acceptation de la variabilité sur les durées hebdomadaires est échangée contre l'acceptation de la norme annuelle, en revanche, l'incertitude du résultat est reportée sur l'année. Cette incertitude, ou les risques de cette codification annuelle, s'étendent jusqu'aux relations de travail. L'accord encadre des pratiques antérieures non formalisées tout en laissant aux usages des marges de manœuvre pour produire et expliciter des solutions variées, peu prévisibles et peu manipulables de l'extérieur et d'en haut. La régulation intermédiaire entre chefs de magasins et salariés relativise ici la régulation d'en haut.

\section{DU TEMPS DE TRAVAIL AU TEMPS DE L'EMPLOI}

Ces quatre histoires du temps de travail nous montrent la complexité de l'analyse des arrangements temporels: la peur du chômage, le statut d'emploi, le rapport de genres et les horizons temporels semblent être des facteurs constitutifs de nouvelles normes temporelles. Parmi ces facteurs la question de l'emploi mérite une attention particulière, car elle nous semble générique de nouvelles inégalités. Ce passage du temps de travail au temps de l'emploi nous montre plus exactement les inégalités dissimulées dans la conception du cadre temporel. Pour comprendre le processus qui met au centre de la négociation du temps de travail l'emploi nous confrontons trois expériences d'accords dans ce domaine (Morin et al., 1998).

Les formes d'engagement d'emploi sont les formes d'action et de représentation que les acteurs engagent dans la négociation du temps de travail. L'objectif est donc ici de sortir l'emploi de sa seule définition économique : «nombre d'heures pendant lesquelles les salariés d'une profession ou d'une région donnée ont été occupés et ont perçu un salaire normal » (Larousse 1988, p. 626, 4). Nous verrons que le rôle que joue l'emploi dans la négociation du temps de travail fait recourir à des engagements caractéristiques que nous souhaitons regrouper sous trois formes rencontrées dans nos monographies: 1'emploi « menace » ou « contrainte », l'emploi « catalyseur », l'emploi « écran ». L'hypothèse faite ici est que les manières d'utiliser l'emploi ont des conséquences tout à fait différentes pour la négociation du temps de travail : l'emploi n'est pas une réalité uniforme qui structure la négociation du temps de travail. L'emploi est associé à un échange au cours duquel 
l'emploi est un moyen de rendre possible la rationalisation de la structure temporelle du travail.

Avec l'entreprise CLARO (que nous allons reprendre en centrant l'analyse sur la question de l'emploi) et le travail du samedi, l'emploi ne concerne pas directement la nature de l'arrangement temporel trouvé, mais bien la condition de son invention : le travail du samedi n'aurait pas pu être négocié sans la «menace » pesant sur une centaine d'emplois dans l'entreprise ; c'est la raison pour laquelle nous proposons d'interpréter, dans ce cas précis, le rôle de l'emploi comme une « contrainte » qui se négocie. En revanche, dans nos monographies deux autres formes d'engagement autour de l'emploi apparaissent : l'emploi « catalyseur » de la négociation du temps de travail et l'emploi comme un «écran » qui bloque la négociation du temps de travail. Dans ces deux cas opposés, l'emploi apparaît d'abord comme le moteur de négociation sur le temps de travail; ensuite, il peut être vu comme un frein de celle-ci. Nous exposerons brièvement ces trois cas.

\section{V.1. L'emploi « contrainte» de la négociation du temps de travail}

L'accord conclu en 1996 chez CLARO se caractérise par un échange entre, d'un côté, la réduction de la durée du travail et le maintien de l'emploi et, de l'autre, l'aménagement des horaires auquel s'ajoute une faible perte de salaire.

Une composante essentielle du contenu de l'accord concerne l'échange désormais classique : réduction de la durée du travail contre aménagement des horaires.

Cet accord met 15 ans pour aboutir, si l'on en juge par sa double acceptation : acceptation $\mathrm{du}$ travail du samedi régulier et acceptation de la perte de salaire accompagnant la diminution de la durée hebdomadaire du travail. Nous avions montré (dans la deuxième partie de ce chapitre) que l'accord donne à l'emploi une place centrale. En effet, le préambule précise d'une part, que cet accord a pour but « de supprimer le plan social engagé et entraînant le licenciement pour motif économique» (de plus de 10 \% des salariés) et « d'augmenter le temps libre et d'améliorer l'emploi ». Nous avions vu que la direction utilise la menace du licenciement pour obtenir le travail du samedi après-midi. De ce point de vue, elle engage avec cette menace un processus de régulation hétéronome du temps de travail. L'accord pourrait alors être considéré comme le résultat provisoire de l'efficacité de la menace (les 100 licenciements du plan social). Dans le langage syndical, l'expression du « chantage à l'emploi » est alors utilisée. Néanmoins, nous ne sommes pas ici exactement dans ce cas de figure, car si la menace est négociable, le chantage ne l'est pas. 
La principale organisation syndicale non signataire et opposée à l'accord a elle-même tiré des avantages de la menace des licenciements concrétisés par le plan social. Vu le niveau de la mobilisation des salariés sous l'impulsion de ce syndicat, seule une menace crédible pouvait infléchir la position du syndicat qui doit justifier son virement de position sans se mettre en porte à faux avec ses propres membres. C'est ainsi qu'il faut comprendre la consultation des adhérents sur une éventuelle participation de ce syndicat à la négociation $\mathrm{du}$ travail du samedi, comme une étape qui permet finalement au syndicat, avec une procéduralisation des licenciements sous forme d'un plan social, de ne pas faire obstruction à l'accord sur le terrain. En échange, l'emploi est maintenu. De plus, en tant qu'organisation non signataire, le syndicat garde un profil bien distinct du syndicat signataire. Cette position lui permettra de continuer à jouer son rôle d'opposition dans l'entreprise à chaque fois qu'il lui semble opportun de le faire. La menace à l'emploi s'exerce donc bien sur les salariés de l'entreprise qui peuvent la percevoir comme un « chantage ». Mais ce chantage est aussi négocié avec les organisations syndicales qui s'en servent pour légitimer leur action : la signature ou la non-obstruction à l'accord. Il faudrait alors plutôt parler d'une contrainte émergeant de la direction mais qui, une fois formalisée (plan social), donne, dans un contexte précis de mobilisation, de la légitimité aux acteurs syndicaux. La menace doit être crédible pour accepter le travail du samedi après-midi. Mais cette crédibilité, comme nous l'avons montré, est négociée avec les syndicats qui restent les acteurs de la négociation : leur comportement différentiel le prouve. La menace de licenciements prépare l'engagement implicite sur l'emploi avec une acceptation du travail le samedi. Emploi maintenu, emploi modifié : le maintien de l'emploi donne donc à cet égard une connotation d'un partage du travail, puisque l'emploi est maintenu en échange du changement de la structure temporelle des activités, en vue d'assurer le temps de la production.

\section{V.2. L'emploi « catalyseur » de la négociation sur le temps de travail}

L'emploi comme menace n'est pas la seule façon de considérer son lien au temps de travail. Il peut être également un catalyseur qui déclenche la négociation du temps de travail. Selon la définition du Larousse (1988) un catalyseur "augmente la vitesse de certaines réactions chimiques sans être consommé lui même». En effet, l'emploi peut dynamiser des négociations sur le temps de travail, sans être consommé lui-même.

Un cas dans le secteur de la santé, nous montre cette forme d'engagement sur l'emploi dans le domaine de la gestion des heures supplémentaires. La négociation porte dans cet exemple sur le repos compensateur : il s'agit d'un accord banal, obtenu dans le cadre d'un 
recours à l'une des dispositions de la loi quinquennale du 20 décembre 1993. Cet accord, signé en 1995 par la direction et l'ensemble des organisations syndicales présentes (CFTC et CGT qui est majoritaire), vise à légaliser un arrangement temporel tacite antérieur. Le résultat de cet accord est moins banal si l'on considère les chemins de la négociation qui révèlent le rôle de l'emploi comme «catalyseur» de la négociation sur les heures supplémentaires.

- Tout d'abord, le recours aux heures supplémentaires avait fait l'objet d'un arrangement temporel tacite entre les salariés et l'employeur: le dépassement du volume d'heures supplémentaires légal était contourné par une transformation de ces heures en repos regroupé sous forme de congés supplémentaires; ces congés pouvant atteindre 12 semaines dans l'année.

- Ensuite, le contexte de l'emploi conduit les organisations syndicales à remettre en cause cet arrangement pour faciliter l'emploi. En effet, une restructuration de l'entreprise en 1993 entraîne une vague de licenciements (100 salariés sur 270); de plus, la réduction programmée des activités (de $5 \%$ par an) fait craindre aux salariés une nouvelle vague de licenciements. D'où un recours à l'inspection du travail à la demande de l'une des organisations syndicales. Le raisonnement est le suivant: "si l'inspecteur du travail constate que l'on fait trop d'heures supplémentaires, il n'y a qu'à embaucher». L’inspection du travail intervient et sanctionne le dépassement des heures supplémentaires. La réponse de la direction n'est pas de favoriser l'embauche mais « puisque l'inspecteur du travail intervient, on devient légal [...], et on supprime le repos compensateur non légalisé ». L'employeur décide alors de remettre en cause les termes de l'arrangement antérieur.

- Enfin, l'accord d'entreprise obtenu se traduit dans une légalisation de l'arrangement tacite : d'une part, il codifie et mesure le recours aux heures supplémentaires qu'il inscrit dans le cadre du repos compensateur de remplacement. D'autre part, il définit de nouvelles règles de recours aux heures supplémentaires, en évitant qu'elles soient comptabilisées dans le contingent légal; mais aussi un nouveau mode de régulation des heures supplémentaires qui, par son caractère légal, devient incontestable.

La signification de cet accord n'est pas évidente : quel rôle l'emploi a-t-il joué ? On pourrait dire qu'il s'agit d'un retour à la case départ, comme le souligne l'employeur : « notre accord, vise à rétablir une pratique antérieure qu'une situation exceptionnelle avait déstabilisé ». Mais ceci n'est pas tout à fait exact, pour deux raisons : tout d'abord, cet accord inscrit le recours aux heures supplémentaires dans un cadre légal et institutionnalise une certaine forme de flexibilité. Ce fait n'est pas sans importance si l'on considère que la 
séquence de travail conduit à travailler de $10 \mathrm{~h}$ à $19 \mathrm{~h}$, alors que les plages horaires officielles sont dans ce secteur de $8 \mathrm{~h}$ à $17 \mathrm{~h}$. Ensuite, l'accord se traduit par un non traitement de la question de l'emploi, pourtant à l'origine de la négociation : l'emploi joue donc un rôle de catalyseur qui déclenche la négociation, sans se retrouver dans le contenu de l'accord. Cette interprétation du rôle de l'emploi s'appuie sur le changement des termes de la discussion: non plus la création d'emplois contre la suppression d'heures supplémentaires, mais le maintien des heures supplémentaires (avec un coût probablement réduit) contre du repos compensateur : dès lors, l'emploi se trouve relégué en dehors du domaine de la négociation en cours. Le catalyseur déclenche donc une réaction et une modification de la structure temporelle. En revanche, l'emploi n'est pas consommé dans cette modification de la structure temporelle et pourtant, l'emploi avait déclenché le processus.

\section{V.3. L’emploi comme «écran»: une négociation du temps de travail bloquée}

L'emploi «menace » et l'emploi «catalyseur» constituent deux formes opératoires distinctes pour négocier le temps de travail. La troisième forme d'utilisation de l'emploi dans la négociation du temps de travail que nous souhaitons introduire, est celle de «l'emploi-écran», provoquant un blocage des négociations du temps de travail. Ce troisième type de relation entre le temps de travail et l'emploi nous est présenté par le cas d'une entreprise comportant plusieurs milliers de salariés, en majorité des femmes, qui occupent des emplois du secteur tertiaire non-marchand. L'accord national stipule en 1991 la réduction de la durée du travail de nuit de 39 heures à 35 heures. Ce même accord, signé par la CFDT, propose simultanément la création au niveau national de 4000 emplois afin de compenser les heures perdues par la réduction de la durée du travail de nuit.

On pourrait penser à une application sans difficultés de cet accord au niveau régional, puisqu'il propose non seulement de réduire la pénibilité du travail de nuit, mais en plus, il promet la création d'emplois dans des quantités non négligeables.

Pourtant, au niveau régional, l'application s'avère difficile. En effet, l'accord national préconise que la création d'emplois est conditionnée par une réorganisation de la journée de travail afin d'augmenter la disponibilité et la productivité du personnel : « La réduction de la durée du travail peut être compensée partiellement par une meilleure adéquation des moyens en personnel à l'activité réelle des services » (avenant à l'accord de 1991). Les horaires de jour sont donc censés devenir plus souples en vue d'accroître la disponibilité des salariés : «Donc, il y aura une journée où l'agent pourrait faire 6 heures de travail au 
lieu de 8 heures, [...], et le surlendemain il pourra faire 10 heures $"$, (entretien avec un représentant de la direction). L'histoire des multiples avenants entre 1991 et 1994 suivant cet accord et un bilan national effectué en 1994 sur la réduction du travail de nuit à 35 heures, prévu initialement pour fin 1993, montre qu'une partie importante des établissements au niveau local n'a toujours pas appliqué l'accord national (environ un sur trois). Dans notre cas, l'application prévue avait provoqué en 1994 des manifestations importantes au sein du personnel.

Pourquoi est-il aussi difficile de réduire le travail de nuit, que l'on sait nuisible pour la santé ?

Il nous semble que dans le cas présent, c'est l'emploi même des salariés qui constitue un écran à l'échange préconisé (réduction de la durée du travail, réorganisation et création d'emplois).

Trois arguments peuvent être invoqués pour comprendre cette situation de l'emploi au niveau régional. Tout d'abord, la réduction de la durée du travail de nuit à 35 heures par semaine et la réorganisation globale impliquent une mesure quantifiée de la durée du travail : " Cela suppose un bagage informatique, or la culture de l'établissement n'est pas au pointage » (représentant de la direction). En effet, la mesure exacte des durées du travail et des opérations sur le plan quantitatif rencontre l'hostilité des salariés qui renvoient à l'administration l'argument d'une qualité de travail, difficilement quantifiable : « voilà ce qu'est l'administratif, il quantifie, il compte en chiffre, en argent, en norme, en kilo, en litre », (entretien avec une salariée).

Le second argument dessine, du point de vue de l'emploi, les limites de l'échange préconisé entre réduction et aménagement du temps de travail. Les horaires de travail obéissent en général aux configurations suivantes : les plages horaires du personnel de jour sont de $7 \mathrm{~h}$ à $15 \mathrm{~h}$, de $8 \mathrm{~h}$ à $16 \mathrm{~h}$ et de $12 \mathrm{~h}$ à $20 \mathrm{~h}$ ( $80 \%$ des salariés $)$. Le personnel de nuit travaille de $20 \mathrm{~h} 30$ à $7 \mathrm{~h}$ ( $20 \%$ des salariés). Alors que la direction souhaite raccourcir le travail de nuit de 10 h 30 à une durée de 8 heures, l'aménagement consécutif des horaires de jour pour l'après-midi de 13 h 30 à 21 h 30 est refusé par les salariés.

La sortie des salariés à $21 \mathrm{~h} 30$ et l'arrivée au domicile à $22 \mathrm{~h} 30$ ne sont pas acceptées par certains qui ne veulent pas utiliser les transports en commun à cette heure tardive (sécurité). D'autres refusent de commencer plus tôt l'équipe du matin à cause de l'absence de transport urbain. De manière générale, ces aménagements nouveaux proposés par la direction sont repoussés parce que la sortie tardive n'arrange pas les femmes et les hommes de service, ni du point de vue des activités domestiques et éducatives, ni du point de vue des activités culturelles : à 22 h 30 , les enfants sont couchés et il est trop tard pour 
profiter des loisirs du soir. L'emploi du temps habituel n'est pas mis en cause par les salariés pour favoriser la réduction du travail de nuit, précisément parce qu'il correspond à l'enchaînement global, mais individuel, des activités sociales : «le personnel de jour ne veut pas qu'on l'embête avec des changements pour arranger le travail de nuit », (entretien avec une salariée). Une des pistes pour comprendre ce comportement concerne la nature des activités.

Le troisième argument concerne directement la réduction du travail de nuit à 35 heures. En effet, il n'est pas sûr que les principaux intéressés de l'accord veuillent réduire les heures de nuit. Le service traditionnel de nuit alterne des semaines longues (5 nuits) avec des semaines courtes ( 2 nuits). Donc, sur 14 nuits, les individus travaillent seulement 7 nuits au maximum. La durée des postes (10 h 30) implique en plus un certain nombre de journées de récupération. Ces récupérations diminueraient avec l'introduction des 35 heures : « avec les 35 heures [...], elles sont obligées de rendre des heures, ce qui veut dire une diminution des récupérations. [...]. Alors pourquoi imposer des horaires à des gens alors qu'ils en sont satisfaits ? (entretien, salariée). L'usage des périodes de récupération rend donc difficile l'acceptation de la réduction du travail de nuit.

L'échange préconisé par l'accord national sur la réduction, l'aménagement du temps de travail et la création d'emplois ne peut devenir effectif au niveau local, car il implique d'abord une certaine "rationalisation » des emplois, notamment autour des mesures du temps des opérations proposées par la direction. En ce sens, il est rejeté par les salariés au nom de leur activité professionnelle. Plus largement, l'emploi des salariés est ici fonction individuelle de l'ensemble des activités sociales aussi bien pour les salariés à plein temps que pour ceux employés à temps partiel et dont l'horaire habituel constitue un arbitrage délicat. Modifier ces horaires voudrait dire mettre en cause l'emploi et son articulation avec les autres activités. L'usage des infrastructure sociales (transports, crèches, commerces, etc.), l'activité professionnelle proprement dite et l'enchaînement des activités multiples font alors de l'emploi des salariés un écran à la négociation du temps de travail.

L'emploi peut d'abord être pensé comme l'insertion ou le maintien de salariés dans l'activité professionnelle, mais aussi comme configuration de l'activité professionnelle autour du statut (plein temps, temps partiel) et comme arbitrage entre des activités sociales reconfigurées par exemple par l'accord sur la réduction de la durée du travail. Notre hypothèse interprétative serait alors que l'irruption de l'emploi dans la négociation collective est une contrepartie dans une logique d'échange qui structure la négociation sur le temps de travail. En revanche, nos trois cas (emploi-régulation, emploi-catalyseur, et 
emploi-écran) nous montrent que ce lien entre le temps de travail et l'emploi n'est pas uniforme. L'emploi « contrainte», comme contrepartie du temps de travail, peut être luimême instrumentalisé en faveur d'un échange plus favorable sur le temps de travail.

\section{Conclusion}

Notre recherche, basée sur l'analyse des chemins de la négociation du temps de travail sur onze sites réels, a montré que le temps de travail constitue une réalité socialement construite par la négociation : il constitue bien le cadre temporel préexistant aux activités professionnelles et visant à les ordonner. Pour autant, ce cadre temporel de travail ne peut être considéré comme une réalité objective, détachée des individus et s'imposant «mécaniquement» voire «naturellement» aux personnes. Ce cadre est le résultat d'une activité de négociation intense et complexe, débouchant sur la production d'un ordre temporel des activités professionnelles. Cet ordre temporel est variable, notamment du fait qu'il est en permanence renégocié : il est établi par convention en fonction de choix qui dépendent des conceptions des négociateurs et des rapports de forces que chacun des protagonistes tente de tirer à son avantage, pour faire valoir ses conceptions du temps et contrôler le modèle rationalisateur du temps de travail. Nous proposons de présenter quelques conclusions.

\section{VI.1. La construction des arrangements temporels}

Premièrement, l'examen attentif du déroulement de la négociation montre l'importance de l'histoire de l'entreprise, du conflit et des tensions qui y règnent, et enfin de l'acteur collectif qui se construit à cette occasion, pour produire ce que nous avons appelé un « arrangement temporel». Tout d'abord, cet examen montre, que la négociation sur le temps de travail a, dans l'entreprise, une longue histoire qui peut s'étaler sur plusieurs années : ce ne sont pas les dispositifs juridiques qui créent la négociation, tout au plus, ils encadrent la négociation dans le triangle flexibilité-emploi-durée, mais en aucun cas ils n'effacent les échecs ou les réussites antérieurs. De plus, chacune des situations révèle que la négociation ne va pas de soi : l'importance du conflit, de la menace, de l'intervention de la puissance publique pour sanctionner une infraction sont là pour en témoigner ; à cela, s'ajoute la concurrence entre les syndicats qui tentent de freiner ou d'accélérer la négociation, en tous cas de contrôler le processus, comme en témoigne le recours au référendum ou bien à des enquêtes d'opinion. Enfin, la variété des solutions retenues porte la marque de ses acteurs : la négociation du temps de travail est l'occasion pour qu'un acteur collectif se mette en place. La configuration de cet acteur collectif est à géométrie 
variable : ce qui frappe, c'est que la position de ces acteurs n'est pas définie une fois pour toutes; d'un côté, les syndicats les plus opposants au travail du samedi ou au travail de nuit des femmes finissent bien par l'accepter tacitement, de l'autre, les cadres commencent à se révolter contre une durée du travail trop élevée et finissent par accepter de signer un accord sur le temps partiel dont ils pourront être les destinataires.

\section{VI.2. Les arrangements temporels comme outil de la gestion de l'emploi}

Deuxièmement, les «arrangements temporels » indiquent que le temps de travail est de plus en plus un outil de gestion du marché de l'emploi : cette conclusion déjà esquissée s'affirme au point de proposer une autre interprétation du temps de travail qui deviendrait le temps de l'emploi. L'emploi est omniprésent dans les négociations sur le temps de travail : il agit, d'une part, sur la définition des règles temporelles qui doivent maintenir l'emploi ou bien permettre à certaines catégories d'accéder à certains emplois (de nuit) ou encore des règles temporelles qui vont de fait distribuer les individus sur des statuts d'emplois différents. L'emploi intervient, d'autre part, sur la régulation du processus de négociation : comme « contrainte », il accompagne la négociation, comme « catalyseur », il la déclenche et comme « écran », il la bloque. C'est sans doute du fait de l'incertitude de l'emploi qu'ils occuperont demain que les salariés acceptent de ratifier un ordre temporel proche de la logique de la production.

\section{VI.3. Le temps de travail incorpore une double rationalité}

Troisièmement, il convient de nuancer le point de vue selon lequel l'ordre temporel des activités professionnelles, en tant que construction négociée, n'obéirait qu'à une seule rationalité qui est celle de la production, comme si les organisations syndicales oubliaient soudainement que les négociations sur le temps de travail ont été développées dans une logique de protection des salariés. L'ordre temporel nous semble porter aussi la marque de la rationalité de la vie quotidienne. L'arrangement temporel incorpore l'ambivalence de la notion de temps de travail qui désigne deux réalités bien différentes : d'un côté, la réalité du temps déterminant et mesurant de manière abstraite les activités professionnelles en vue d'une performance productive ; de l'autre, la réalité du temps qualitatif, renvoyant aux activités concrètes, appréhendées de manière personnelle, visant à ordonner leurs activités en vue d'obtenir une certaine cohérence et concordance dans leur temporalités : le refus par les salariés du secteur de la santé de négocier sur la réduction de la durée du travail, en témoigne. La négociation ne réussit en aucune manière à transformer le temps qualitatif et subjectif en un temps quantitatif et objectif : elle rend possible la construction du temps de 
travail comme temps hétéronome, c'est-à-dire comme cadre temporel dans lequel les activités professionnelles sont inscrites. Que ce cadre ait pu être négocié ne doit pas laisser croire qu'il est contrôlé par la personne qui travaille : dès lors que « le temps prévaut sur la tâche » selon l'expression de Naville (1972), l'homme qui perd la maitrise du temps et le contrôle de son processus d'action, est affaibli.

Il nous reste cependant à déchiffrer un peu plus ce qu'est ce «temps arrangé » qui est localement « bricolé » et qui met en tension le temps du travail et le temps de l'emploi, le temps des activités professionnelles et celui qui ne leur est pas consacré, le temps collectif et le temps individuel de chacun. Il nous faut explorer plus comment les individus se représentent le temps de travail pour comprendre pourquoi ils aboutissent à de tels arrangements temporels.

« L'arrangement temporel » s'inscrit plus largement dans un espace de négociation dont il est le résultat provisoire. Un lieu où peuvent s'observer des significations qui renvoient aux projets des acteurs. De ce point de vue, l'espace de négociation du temps est débordé par les conceptions du temps des acteurs. De plus, les limites de cet espace de négociation peuvent être source de sécurité et délimiter un champ, parce qu'il définit un espace d'action pour un temps donné. Néanmoins, cette sécurité que confère un espace de négociation peut être trompeuse. L'arrangement n'est pas durable et il porte généralement le prochain conflit qui entraînera la négociation suivante. D'autres caractéristiques de l'espace de négociation sont alors à prendre en compte pour l'analyse : sa dimension cognitive et les représentations que les acteurs se font du temps de travail. C'est l'objet du chapitre suivant que d'analyser ce « temps représenté ». 


$$
\begin{gathered}
\text { CHAPITRE IV } \\
\text { LES REPRESENTATIONS DU TEMPS : }
\end{gathered}
$$

LE REFERENTIEL DE L'ACTION SYNDICALE 


\section{Introduction}

Le temps de travail entre en France depuis une quinzaine d'années dans le champ de la négociation collective. Lorsqu'il devient objet de négociation, il est inscrit dans un champ déjà constitué, celui des relations professionnelles. De ce point de vue, le temps de travail ne subit-il pas les marques de cette inscription ? Cette irruption dans un espace social déjà structuré ne limite-t-elle pas les discussions et les acteurs possibles? Les processus qui jalonnent la négociation des autres thèmes (comme les salaires) ne vont-ils pas marquer la nature des échanges et des compromis? Les positions des acteurs, sédimentées dans d'autres espaces, ne vont-elles pas s'affronter dans les controverses? Les positions des acteurs syndicaux ne sont-elles pas déjà prédéfinies ? Y a-t-il du jeu dans cette nouvelle règle du jeu?

Il pourrait paraître un peu tardif de n'aborder la question du temps négocié qu'après avoir analysé les pratiques locales. Il nous a semblé pertinent de caractériser d'abord le contexte de la négociation en montrant que le temps de travail est le dernier thème qui fait son entrée dans la négociation collective : au cours du chapitre II, nous avons montré que le contexte de la négociation est tout à fait singulier puisque les termes de l'échange supposent l'acceptation de la flexibilité contre l'aménagement et la réduction du temps de travail. De plus, nous venons de montrer (dans le chapitre III) que l'espace de négociation n'est pas «verrouillé »: des arrangements temporels variés prennent place dans des situations singulières. Les termes de l'échange ont fait apparaître l'importance de l'emploi : l'acceptation de la flexibilité doit être entendue sous réserve d'un engagement à maintenir l'emploi.

Il nous semble à ce stade que la rationalité de l'action syndicale doit être explicitée. Comment les organisations syndicales de salariés arrivent-elles à conserver leur identité et leurs prérogatives dans un univers de négociation qui semble être contrôlé par les employeurs? Comment les organisations syndicales peuvent-elles encore orienter leurs actions dès lors que leur stratégie apparaît « encapsulée » dans l'ordre de la production?

Pour répondre à ces questions, nous aborderons la négociation collective sur le temps de travail de façon à expliciter l'articulation entre des comportements de négociation et les référentiels sur lesquels ils se fondent. Dès lors, on se donne les moyens d'explorer les contradictions et la dynamique de l'action syndicale. 
Considérer que la négociation du temps de travail constitue un objet de recherche pertinent repose sur la conviction que cet objet va quelque peu modifier la négociation collective, ses déterminants, ses formes, ses modalités et ses enjeux. Au départ de cette recherche, il y a pour nous une hypothès $\mathrm{e}^{28}: \mathrm{du}$ fait que le temps de travail a une histoire dans le domaine de la négociation, il pourrait être l'occasion d'ouvrir un espace de discussion et de confrontation alors que cet espace était «verrouillé » par l'hégémonie de l'État comme seule puissance normative légitime. Le temps de travail pourrait être l'occasion d'une reconfiguration de l'action syndicale ; l'occasion de faire émerger de nouvelles alliances et de nouvelles exclusions. 


\section{SYNDICALISME ET NEGOCIATION COLLECTIVE DU TEMPS DE TRAVAIL}

Dans un premier temps, nous proposons de donner quelques caractéristiques du champ dans lequel le temps de travail va prendre place; dans un deuxième temps, nous rappellerons quelques manières de voir l'action syndicale en sociologie. Enfin, dans un troisième temps nous nous consacrerons à l'analyse des référentiels négociatoires.

\section{I.1. Négociation collective et régulations sociales}

La négociation du temps de travail en entreprise s'insère dans les pratiques sociales mises en œuvre par des groupes sociaux à l'occasion du travail salarié. L'emprise de la vie quotidienne sur la vie professionnelle (famille, amis, travail domestique et loisirs) peut amener les acteurs en entreprise à écarter ou à favoriser l'émergence de nouvelles normes de temps : dans un cas présenté au chapitre II, le travail du samedi est refusé pendant plus de 10 ans parce qu'il implique la mise au travail pendant un temps réservé aux activités familiales et de loisir; dans un second cas, le temps partiel pour les cadres est introduit parce qu'il constitue une soupape aux pressions qui pèsent sur les cadres avec des durées du travail jugées « excessives »; dans un troisième cas, le travail de nuit des femmes est introduit et légitimé par les nécessités productives et reproductives et par l'aspiration des femmes à l'égalité dans le domaine du travail professionnel. En effet, les syndicats et les organisations patronales ne restent pas les seuls acteurs bien qu'ils aient juridiquement et politiquement le monopole de la négociation collective. L'intervention de l'État et de groupes de salariés qui ne se confondent nullement avec les organisations syndicales peuvent amener les syndicats et la direction de l'entreprise à modifier leur position sur l'organisation du temps de travail. Face à cette perspective, la «négociation collective » et les « relations professionnelles » ne constituent pas le substrat des rapports sociaux ou un système social homogène autour de ceux dont on reconnaît le droit à la parole et à l'action. Le terme de « relations professionnelles » couvre donc un domaine plus large. Comme le note Lallement (1996, p. 3), avec le terme de « relations professionnelles », « l'on désigne habituellement l'ensemble des pratiques et des règles qui dans une entreprise, une branche, une région ou l'économie toute entière, structure les rapports entre les salariés, les employeurs et l'État». Ce qui nous intéresse, c'est de comprendre du point de vue sociologique comment les acteurs s'associent pour construire un arrangement temporel, c'est d'expliciter leurs contraintes et leurs décisions. Au fond c'est la confrontation des projets de règles et les régulations qui les accompagnent (Reynaud, 1989, 1996) qui sont au centre de nos préoccupations. La négociation collective rend-elle plus légitime 
l'arrangement temporel? Dans ce champ, l'analyse du comportement des organisations syndicales nous semble particulièrement intéressant. Que peut-on dire à partir d'une analyse du rôle de l'acteur syndical dans la négociation collective sur le temps de travail ? Certes, il approuve globalement les accords sur le temps de travail, mais nous pouvons rappeler que la «négociation collective » est marquée par les traces d'une réalité sociale beaucoup plus riche que ne le laisse entendre la codification écrite de l'accord final. Néanmoins, les acteurs détiennent le monopole de la négociation institutionnalisée et décident des codifications du temps de travail. Il s'agit de retrouver où et comment, tout au long du chemin de la négociation parcouru pour parvenir à l'accord, l'acteur syndical se divise ou s'associe. Les formes variées des temps de travail ne rendent-elles pas plus difficiles la formulation de revendications collectives sur des dispositions communes à tous les salariés?

Nous nous interrogeons avant tout sur les formes de régulations mises en place. La négociation de nouvelles règles du temps de travail change-t-elle la manière de parvenir à un accord? Peut-on parler de formes de régulation multiples et quelle est la place des acteurs syndicaux dans celles-ci ? Quelle est leur maîtrise du processus de définition du temps de travail ? A quel rationalité obéissent-ils ? Leur actions et leurs représentations constituent-elles un ensemble cohérent qui renvoie à une structure de pratiques?

\section{I.2. Le temps de travail et l'action syndicale}

Deux ouvrages portant sur l'analyse du syndicalisme français confirment la complexité de la construction de l'organisation syndicale. Les travaux de Groux et Mouriaux (1989 et 1992) sur la CFDT et la CGT montrent que l'analyse sociologique du syndicalisme ne peut pas faire l'impasse sur la constitution historique du syndicalisme. A travers l'exemple de la CFDT et de la CGT, les auteurs montrent que chaque organisation est tributaire de son héritage, de son organisation interne, de conflits charnières et des périodes d'orientations stratégiques. L'évolution du contexte sociétal et politique influe fortement sur la conception de l'action syndicale. En s'inscrivant dans la problématique portant sur les formes de régulation du syndicalisme proposée par Reynaud, Bernoux, Lavorel (1966), Groux et Mouriaux (1989, p. 251) caractérisent la nature de l'action syndicale selon les organisations. Ils estiment que le syndicalisme de la CFDT reste un syndicalisme de régulation fondée sur la négociation «à froid» et sur des pratiques de concessions mutuelles en vue du maintien des équilibres économiques et sociaux, tandis que la CGT serait passée d'un syndicalisme de masse à un syndicalisme de crise plus complexe avec 
une mise en cause du syndicalisme de lutte des classes «traditionnel» (Groux \& Mouriaux, 1992, p. 260). La question du temps de travail illustre les différentes conceptions du syndicalisme dans la période qui précède les lois Auroux. Ainsi la CFDT décide dès 1979 de proposer une réduction massive de la durée du travail à 35 heures (Groux et Mouriaux, 1989, p. 210). Cette conception du syndicalisme illustrée par la question du temps de travail se traduit dans la négociation collective par des propositions qui constituent dans leur cohérence globale, une « position médiane entre celle du CNPF et celle de la CGT» (op. cité, p. 211). La CFDT propose en 1979 non seulement une variation possible de la durée hebdomadaire du travail entre 38 et 42 heures (40 heures en moyenne), anticipant ainsi les premières formes de modulation du temps de travail, mais elle propose aussi la limitation de la durée annuelle du travail de 1920 heures à 1800 heures ainsi que la réduction de la durée hebdomadaire à 38 heures pour les travaux pénibles (op. cité). «La CGT revendique, pour sa part, l'instauration immédiate des 38 heures et un plafond de 1717 heures maximum par an, voire 1582 heures pour les travaux pénibles et 1518 heures pour le travail posté »(op. cité). Dans cette période précédant les lois Auroux, la question de l'échange entre l'aménagement du temps de travail et la réduction de la durée du travail occupe déjà les négociateurs. En 1980, la CGT refuse ; la CFTC critique l'extension du travail le dimanche et FO pense que le patronat profite davantage de ces propositions que les salariés (op. cité).

Le cas de la CGT sera pour Groux et Mouriaux (1992) l'occasion d'affiner le raisonnement sur le syndicalisme et de montrer en détail que plusieurs formes de syndicalisme semblent pouvoir cohabiter au sein d'une même organisation. Trois formes de syndicalisme sont présentées (op. cité, pp. 211-221) : le «syndicat-mouvement », le « syndicat-recours » et le « syndicat-institution ».

Pour le cas de la CGT, les «formes-syndicat» montrent au fond la complexité de l'organisation. Mais, comme le notent les auteurs (op. cité, p. 218), rien n'est figé historiquement et le passage d'une forme-syndicat dominante à une autre dans le cas concret permet en effet d'analyser la dynamique du changement social.

L'exemple de la CGT et celui de la CFDT montrent fort bien que les formes de syndicalisme semblent avoir une certaine cohérence d'ensemble. 


\section{I.2.A. Le temps de travail comme pivot de l'action syndicale}

Le syndicat est d'abord une organisation avec une réalité historique, des codes partagés, des frontières établies, des alliances tissées et il est composé de salariés d'origine et de statuts divers. Comme le montre le syndicalisme français, le pluralisme des acteurs et des stratégies est source de conflits, en particulier de conflits entre idéologies à long terme et intérêts immédiats: «En 1981, comme en 1945-1946, les syndicats sacrifièrent les revendications immédiates à leur idéologie : la reconstruction d'une France indépendante des Etats-Unis en 1945 et le succès du gouvernement socialiste en $1981 »$ (Tiano, 1988). Ces idéologies en tant que systèmes d'idées et de projets se traduisent en pratiques syndicales distinctes, évoluent sous la pression interne (position de l'organisation dans le syndicalisme français) et externe (réactivité et adaptation au changement de l'environnement). Le temps de travail en tant qu'objet de négociation permet de distinguer les référentiels négociatoires des syndicats : ce thème implique non seulement l'adoption d'un minimum de principes de négociation qui se sont développés fortement depuis les années 80, mais plus encore la mobilisation de l'ensemble de la mémoire organisationnelle. Dans un des rares travaux sur le syndicalisme et le temps de travail, Autrand (1988) montre que le temps de travail constitue en effet le meilleur révélateur de la différenciation idéologique et stratégique des syndicats; il indique, pour l'exemple français, que cette différenciation et cette division syndicale ont été un processus relativement lent. Les référentiels négociatoires se sont constitués lentement. A la fin des années 70, l'unité syndicale autour de la revendication de la réduction de la durée du travail à 35 heures par semaine se fissure malgré un consensus relatif au niveau du mouvement syndical européen : cet éclatement donne lieu à des argumentations différentielles et des polémiques face à la position du patronat. La polémique intersyndicale autour de l'aboutissement à une réduction de la durée du travail se traduit par des priorités différentes. FO revendique l'allongement des vacances et les 35 heures situées au niveau européen. La CGT demande les 35 heures avec compensation intégrale des salaires et passage immédiat à 38 heures. La CFDT demande au congrès de 1979 l'application des 35 heures «multidimensionnelles », sans compensation intégrale et elle accepte l'annualisation et diverses mesures de flexibilité afin de développer le temps libre et la vie associative.

Selon Autrand (1988), la diversification des stratégies est très avancée entre 1981 et 1983, comme le montrent les résultats et l'attitude des syndicats face aux lois Auroux. La cinquième semaine de vacances, les 39 heures, la limitation des pouvoirs de l'inspection du 
travail révèlent la rupture entre les syndicats. La décentralisation de la négociation et la possibilité d'accords dérogatoires affaiblissent le référentiel négociatoire partagé. La CFDT proclame désormais le partage du travail, tandis que la CGT et FO adoptent une attitude de retrait. Après 1984, le gouvernement abandonne la perspective de la réduction de la durée du travail, mais la CFDT et la CGT continuent à revendiquer les 35 heures sans pour autant envisager des actions communes. La CGT semble s'aligner sur la lancée de l'action des syndicats allemands (revendication des 35 heures dans la métallurgie) et la CFDT tente des négociations à froid avec le CNPF : ce dernier propose un accord portant entre autres sur l'intérim, les contrats à durée déterminée et le travail à temps partiel. La CFDT est alors désavouée par les fédérations et les unions régionales. Autrand (1988) constate que les divisions inter et intra-syndicales renvoient, dans cette période, à la faible institutionnalisation de la négociation collective. La défaite du mot d'ordre des 35 heures met néanmoins en avant la recherche de nouveaux modes d'exercice du contrôle syndical.

En résumé, nous pouvons observer que les représentations, les positions, les alliances et les exclusions entre syndicats semblent être primordiales dans la compréhension de la négociation du travail et dans l'émergence de mouvements sociaux. Les stratégies et les idéologies des syndicats face à ces objectifs semblent en effet varier d'une organisation à l'autre, d'un contexte l'autre.

\section{I.2.B. Pourquoi s'intéresser à la négociation du temps de travail ?}

D'abord parce que les négociations indiquent la capacité des acteurs à débattre d'une question essentielle qui les divise avec pour les uns la nécessité de retrouver une « concordance des temps» et pour les autres la nécessité d'augmenter la flexibilité de la production. Entrer en négociation, c'est accepter de construire une espace de discussion pertinent pour débattre dans un contexte précis de la question de l'ordre temporel.

Ensuite parce que ces négociations indiquent la capacité des acteurs à construire un cadre temporel de travail légitime : certes, il s'agit de solutions communes qui n'ont pas la même force coercitive et rien ne préjuge des modalités de mise en œuvre. Ce cadre temporel est le résultat de compromis, de concessions réciproques et de contreparties qui n'ont de sens et de portée que dans un contexte localisé : le cadre temporel a une cohérence locale.

Enfin parce que ces négociations indiquent que les parties en présence s'accordent sur un ordre temporel qu'ils s'engagent à respecter : le cadre temporel a une certaine robustesse puisqu'il a été conçu pour être mis en œuvre. Bien entendu ce contrat est fragile : tous les 
syndicats ne sont pas signataires et l'usage de ce cadre nécessite une interprétation et une adaptation aux situations de ses destinataires.

En conséquence, nous pensons que la négociation du temps de travail aboutissant à des accords produit un équilibre provisoire basé sur un ordre temporel en transition. Pour autant, il nous reste à explorer la solidité de cet accord: la négociation aboutit-elle à réduire l'asymétrie des représentations du temps de travail que véhiculent les différents acteurs?

La négociation conduit-elle à des accords reposant sur une représentation unique du temps de travail ? Certainement pas. L'analyse des pratiques de construction du cadre temporel montre que ce cadre est conçu davantage pour favoriser la flexibilité de la production que pour améliorer les conditions de vie des salariés, même si la flexibilité est échangée contre le maintien de l'emploi. Il révèle la nature des compromis temporels élaborés. Cette analyse pourrait nous amener à conclure que les organisations syndicales sont prisonnières de cette logique tournée vers la flexibilité. L'étude monographique (voir le chapitre III) permet de douter de cette affirmation. Rien n'indique que les conduites syndicales obéissent à une conception d'un temps de la production abstrait et mécanique qui mène à une vision d'un temps vide de contenu. Les chemins qu'emprunte la négociation sur le temps de travail rappellent la dualité des rationalités en présence et des tensions entre les acteurs : un arrangement temporel ne s'impose pas mécaniquement, mais il est construit par confrontation, par négociation et par conflit. C'est dire qu'on ne peut pas interpréter les compromis temporels comme des solutions temporelles exclusivement tournées vers la flexibilité de la production: les arrangements temporels dans leur finalité, dans leur contenu ou dans leur mode de construction cristallisent cette dualité de logiques et cette tension entre les acteurs.

\section{I.2.C. Le référentiel de négociation : comment interpréter la nature, la portée et la solidité des accords?}

Sur quelle vision du temps de travail reposent les accords? Nous pensons que le sens d'un accord dépend du référentiel temporel construit dans la négociation : il s'agit d'un schème opératoire qui correspond à l'ensemble des représentations et des connaissances engagées et mobilisées dans l'action négociatoire sur le temps de travail. Il s'agit donc d'un référentiel de situation dans la mesure où c'est le contexte variable de l'action qui décide de son contenu. 
Un accord sur le temps de travail repose sur un référentiel de situation dans lequel par exemple la flexibilité est échangée contre le maintien de l'emploi ; en revanche un accord ne suppose pas que les parties en présence aient une vision commune du temps de travail. Les organisations syndicales ont donc chacune une manière de voir le temps : un référentiel singulier qui est un référentiel d'action c'est-à-dire lié à des finalités et valeurs engagées dans la négociation du temps de travail.

En revanche, parallèlement et paradoxalement à cette première manière de voir le temps, les organisations syndicales partagent un même référentiel commun autour de quelques principes. Ce référentiel commun vise à préserver la stabilité des cadres temporels. Il vise à mieux gérer les temps sociaux : il est commun à l'ensemble des organisations syndicales et indépendant de la situation de négociation. Il incarne les représentations les plus profondément ancrées du temps de travail et des temps sociaux, mais paradoxalement, dans la négociation en formes que nous avons vu au chapitre précédent, il est le moins opératoire des deux référentiels.

\section{DYNAMIQUe ET CONTRADICTION DE L'ACTION SYNDICALE}

Le temps de travail devient objet de négociation : cela signifie qu'un espace de négociation auparavant verrouillé s'ouvre à la discussion et à la confrontation des points de vue. Cela signifie-t-il que cette négociation engendre un acteur collectif? Quels en sont les membres? Sur quelles positions s'accordent-ils? Ont-ils un projet commun ? C'est à ces questions qu'est consacrée cette partie.

Pour cela, nous proposons d'explorer le référentiel négociatoire, c'est-à-dire l'ensemble des représentations, des connaissances, des expériences, des croyances et des valeurs détenues par les syndicats et mobilisées à l'occasion de la négociation. L'acteur syndical n'est pas homogène. Pour autant, cela ne signifie pas qu'ils sont privés d'action commune ou de projet commun ; mais cette action commune ne peut se développer sans un minimum d'accord sur la question du temps de travail. L'analyse des référentiels négociatoires doit nous permettre d'expliciter la nature des conditions de cette action commune. Nous proposons donc d'analyser le référentiel négociatoire d'un double point de vue. Premièrement, du point de vue des situations concrètes, le cours de la négociation révèle des oppositions entre syndicats, voir des alliances partielles et locales. Nous appellerons référentiel de situation, les représentations et valeurs qui sont mobilisées pour la négociation; dès lors qu'il y a mise en acte des valeurs dans une situation concrète, le 
référentiel de situation qui en résulte varie d'un syndicat à l'autre. Deuxièmement, du point de vue de leurs orientations fondamentales, les syndicats ont en commun un certain nombre de valeurs liées au maintien de l'emploi et à la défense de la santé et du bien-être des salariés, sans pour autant nier totalement les valeurs liées à la logique du marché.

Dans une première partie (2.1), nous caractériserons les pratiques syndicales du point de vue des comportements de signature des accords : nous chercherons les régularités dans les positions syndicales, les points communs entre syndicats, mais aussi les différences. Dans une seconde partie (2.2), nous préciserons le référentiel de chacun des syndicats, les valeurs auxquelles ils obéissent et les schémas de pensée qui ordonnent leurs actions. Enfin, dans une troisième partie (2.3), nous envisagerons l'action syndicale à partir d'un référentiel commun basé sur des valeurs communes que partagent les différents syndicats à propos du temps de travail.

\section{II.1. La distinction des pratiques syndicales}

Les pratiques syndicales sont caractérisées ici par le comportement des acteurs syndicaux face à la signature ou non de l'accord. Bien entendu cette réduction des pratiques syndicales aux comportements de signature comporte deux limites : premièrement, les accords ne «remontent» pas tous auprès des Directions Régionales du Travail; deuxièmement, les pratiques syndicales sont explicitées à partir des accords. Or, d'autres situations dans lesquelles il n'y a pas d'accords, mais des quasi-accords ou des conflits auraient pu nous informer sur les pratiques effectives. Nous avons cependant considéré que le comportement de signature était un bon indicateur de la pratique syndicale : il ratifie une position à l'égard d'un domaine et d'une situation, il désigne ceux avec qui on s'allie et ceux à qui on s'oppose ; au-delà de la logique de positionnement et de la logique de l'alliance, il renvoie à des représentations et à des croyances et n'est donc pas réductible à un positionnement opportuniste, même si l'on ne peut exclure le poids du contexte dans cette prise à partie.

L'analyse du comportement de signature peut être présentée sous deux aspects : d'abord un comportement de signature qui témoigne d'un engagement intense des syndicats dans la négociation du temps de travail; ensuite un comportement différencié selon les organisations syndicales et circonstancié selon les thèmes. 


\section{II.1.A. Du comportement de signature à l'engagement dans la négociation}

La signature syndicale (ou à l'inverse le taux de refus de signature) peut révéler la légitimité des accords. Ainsi, ce sont les délégués des cinq organisations syndicales représentatives qui peuvent ou non signer un accord sur le temps avec la direction de l'entreprise ou de l'établissement. Pour nos deux périodes d'observation des accords régionaux (une période longue de 1984 à 1994 et une période courte de 1994 et 1995), nous avons donc d'abord calculé le taux de refus de chaque organisation syndicale portant sur certains thèmes du temps de travail. Ce taux de refus indique le nombre de signatures sur l'ensemble des accords signés en matière de temps de travail dans lesquels le syndicat est représenté. Ce taux est à mettre en relation avec le taux de présence du syndicat pour l'ensemble des accords conclus.

Le résultat principal de ce traitement quantitatif de la signature syndicale est que le taux d'acceptation des accords sur le temps de travail est élevé, comme nous l'avions vu précédemment. Néanmoins, nous constatons que l'adhésion syndicale aux accords s'est considérablement atténuée depuis deux ans.

L'adhésion syndicale aux accords oscille pour les années 1984-1993, entre $81 \%$ et $96 \%$ selon l'organisation syndicale, mais elle s'est néanmoins affaiblie lors des deux dernières années. Pour les années 1994-1995, la CGT passe d'un taux de refus de 18,9\% à 31,5\% et FO passe de 5,5\% à 13,3\%. La CFDT refuse maintenant $16,9 \%$ des accords au lieu de $7,9 \%$ pour la période précédente. La CGC passe de 3,9\% à $10 \%$ de refus. Seule la CFTC voit son taux de refus diminuer: il passe de $7,5 \%$ à $2,6 \%$ des accords après la loi quinquennale. Ces résultats sont à nuancer avec les taux de présence des syndicats sur l'ensemble des accords. Ainsi la CGT est présente dans 63,6\% des entreprises ayant conclu des accords après la loi quinquennale. La CFTC par contre n'a de représentants que pour $27,9 \%$ de l'ensemble des accords conclus. La figure 5 donne le taux de refus par organisation syndicale de la signature d'un accord donné (1984-1993) et son évolution (1994-1995) à condition qu'elle soit représentée dans l'entreprise concernée. 


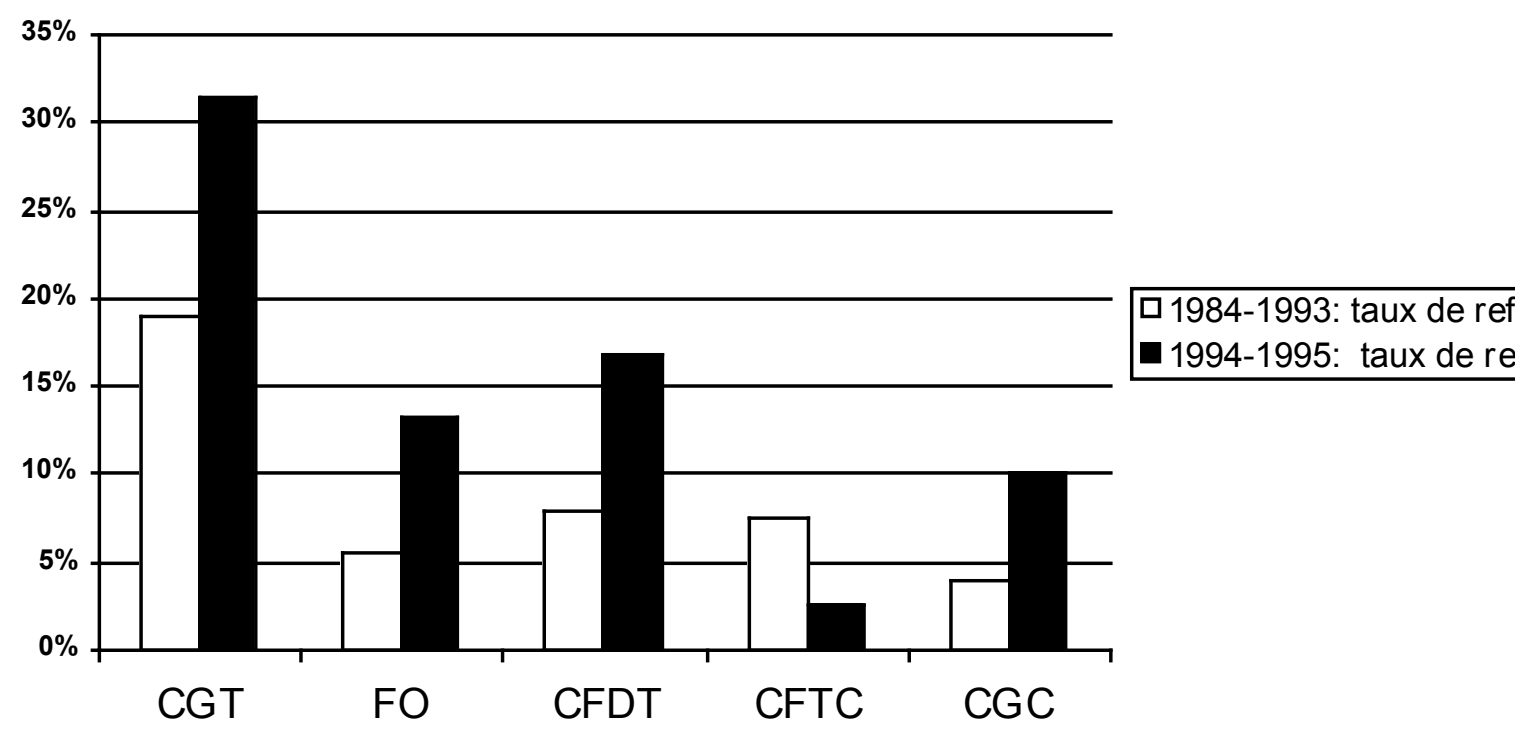

Figure 5: Taux de refus de signature des accords avant et après 1994

Dans cette première analyse du taux de signature, les comportements se distinguent dans le temps (progression du refus pour la dernière période) et d'une organisation à l'autre. Dans l'ensemble, nous constatons une approbation forte. La Confédération française des travailleurs chrétiens (CFTC) avec $2 \%$ de refus et la Confédération générale des cadres (CGC) avec $3 \%$ de refus signent quasi systématiquement tous les accords. La Confédération française démocratique du travail (CFDT) et Force Ouvrière (FO) refusent de signer un accord sur 10 (respectivement $9 \%$ et $11 \%$ de refus), alors que la Confédération générale du travail (CGT) en refuse un accord sur 4 (24\% de refus).

\section{II.1.B. Un engagement dans la négociation différent selon les syndicats et les thèmes}

Au niveau national, le bilan de la négociation collective du temps de travail (1997) ${ }^{29}$ révèle qu'après une progression forte et continue en 1993 et 1994, l'année 1995 constitue un tassement, l'année 1996 le mouvement général à la hausse continue $(+12 \%$ d'accords par rapport à 1996). En 1996, plus de 4000 accords d'entreprises sur le temps de travail ont été conclus. Le bilan de la négociation collective $(1996)^{30}$ montre que 6 accords sur 10 portent sur l'aménagement du temps de travail. La modulation-annualisation, le temps

29Ministère du travail et des affaires sociales (1997). La négociation collective en 1996, Paris: La documentation française, tome 1.

30 Ministère du travail et des affaires sociales (1996). La négociation collective en 1995, Paris : La documentation française, tome 1 . 
partiel, et le temps partiel annualisé constitue des formes fortes de la négociation du temps de travail.

Notre étude montre qu'au cours de la période 1984-1995, il y a bien une variabilité selon les thèmes. Les thèmes retenus sont ceux que nous avions retenu dans les accords lors de l'interprétation de la structure temporelle par classes : le temps échangé (réduction du temps de travail et extension de l'utilisation des machines), le temps des marchés (modulation-annualisation), le temps individualisé (temps partiel) et enfin le temps des machines (travail en équipes et en fin de semaine).

Nous pouvons constater que le thème des « équipes de fin de semaine » est celui qui atteint le refus de signature le plus important ( $18 \%$ ) toutes organisations syndicales confondues. Le travail de nuit est refusé en moyenne dans $15 \%$ des cas (un accord sur 7). Un accord sur 10 portant sur le travail à temps partiel et/ou la modulation n'est pas signé (11\%). Et les syndicats limitent leur refus pour la réduction de la durée du travail à $8 \%$ (10 accords refusés sur 132).

Trois groupes d'acteurs syndicaux se dessinent dans ces résultats :

1) La CGT pratique plus couramment le refus de la signature : $36 \%$ de refus pour les équipes de fin de semaines et $30 \%$ pour le temps partiel.

2) FO et la CFDT refusent ponctuellement des accords : FO refuse davantage le travail de nuit (21\% des accords) et la CFDT signe moins sur les équipes de fin de semaine (22\% des accords).

3) Enfin la CFTC et la CGC ne refusent que quelques accords très localisés.

Le référentiel dans ces négociations ne peut donc être le même d'une organisation à l'autre, car autrement comment pourrait-on comprendre que la CGT refuse presque un tiers des accords sur le temps partiel alors que FO n'en refuse que $4 \%$. La division en différentes organisations syndicales conduit bien à une relative autonomie dans le comportement négociatoire.

En conclusion, on peut dire que des types de la négociation du temps de travail cohabitent avec des conceptions syndicales particulièrement variables pour chaque type. Les organisations syndicales sont partagées sur les processus de la déconstruction du temps de 
travail : ces processus sont plus ou moins approuvés selon les organisations syndicales. En revanche, il apparaît une hiérarchie implicite de l'approbation de ces processus : le temps des machines est davantage refusé que le temps partiel ou la modulation-annualisation et la réduction de la durée du travail bénéficie de l'approbation la plus importante.

\section{II.2. Des référentiels négociatoires singuliers}

Nous proposons d'expliciter les distinctions, d'une part, par des représentations, et d'autre part, par de l'expérience sédimentée et constitutive de la mémoire de l'organisation syndicale. Dans ce creuset des représentations du présent, du passé et du futur s'élaborent des comportements concrets. Comment passer de l'un à l'autre ? Comment articuler ce qui se donne à voir immédiatement, tel comportement de signature, avec ce qui est caché, obscur, parfois même difficile à expliciter, comme le poids du passé et de la mémoire syndicale?

Nous avons analysé ici les organisations syndicales les unes après les autres : chacune est prise isolément pour tenter de caractériser ce qui oriente son comportement, les valeurs qui ordonnent ses pratiques, les imaginaires qui s'entremêlent avec les situations concrètes. Par ce voyage au centre de l'organisation syndicale, nous tenterons d'expliciter, au-delà des slogans manifestes, ce qu'il y a de plus intime, de plus spécifique, de plus interne à l'organisation. Sans jugement et sans a priori sur la légitimité des propos tenus, nous tenterons de comprendre ce qui fonde la position concrète, même si ce fondement est parfois hésitant et issu d'histoires lointaines.

Pour ce travail d'explicitation, nous avons procédé (1995-1996) à des entretiens de deux types avec les différents responsables syndicaux. D'abord, des entretiens en situation avec ceux qui sont engagés dans l'action négociatoire courante et que nous avons rencontrés au cours de l'étude monographique, soit au total une vingtaine de personnes. Ensuite, des entretiens hors situation locale, avec tous les responsables syndicaux régionaux, avec qui nous avons eu nous avons eu des entretiens classiques. De ce matériau, nous tenterons d'extraire le référentiel de chaque organisation syndicale. Observons ici aussi que cette étude se limite à une région et que les résultats ne sont pas généralisables sur le niveau national. Ainsi que dans une autre région l'étude des référentiels syndicaux pourrait amener d'autres éléments de compréhension. Notons enfin que les référentiels du temps de travail peuvent évoluer très vite, comme le montre la période actuelle de négociation sur la base de la loi Aubry sur les 35 heures. Dans ce sens la présentation des référentiels du 
temps de travail ne peut être qu'une photographie locale des représentations qui accompagnent la pratique syndicale.

Le référentiel d'une organisation syndicale est l'ensemble des valeurs, des connaissances, des expériences, des croyances que partagent les membres de l'organisation. Ces composantes sont ordonnées et hiérarchisées de façon spécifique dans chaque organisation. Nous en proposons une présentation schématique :

- La CFDT est favorable au partage du travail et à la flexibilité contrôlée.

- La CFTC met en avant la solidarité et se replie sur la protection de la famille.

- La CGC se fixe sur la semaine de 4 jours mais accepte la flexibilité.

- La CGT revendique le plein-emploi et la stabilité des repères temporels.

- Enfin, FO veut promouvoir la contractualisation sur le temps de travail, face à la guerre économique.

De nos entretiens avec les syndicalistes en entreprises et dans les Unions régionales, nous proposons ici d'extraire quelques caractéristiques du référentiel des organisations syndicales: les mots-clefs caractérisant les syndicats, leurs attitudes par rapport aux questions de la flexibilité et de la réduction de la durée du travail. Cette présentation n'a pas pour objectif d'opposer les discours aux faits (les accords signés), mais il s'agit plutôt de comprendre face aux comportements négociatoires comment l'organisation voit son action propre.

\section{II.2.A. La CFDT : partage du travail et flexibilité contrôlée}

Les mots-clefs caractérisant l'organisation sont : "solidarité, opposition et négociationaction ».

La CFDT constate après une tournée dans les unions départementales que le thème de la réduction de la durée du travail n'est pas toujours ressentie comme une nécessité, alors qu'elle attribue trois vertus à celle-ci :

«1) Relancer en réinsérant des chômeurs, relancer l'économie notamment les jeunes qui sont pas mal exclus. 2) Avoir du temps pour soi et du travail pour tous. En fait, avec une réduction du temps de travail, c'est des salaires et des chômeurs qui sont gagnants dans l'affaire. 3) Libérer du temps pour les uns doit se traduire par du travail pour les autres, 
mais en réorganisant le temps du travail, tout en prenant en compte les besoins d'adaptation et de production des entreprises ».

La réduction de la durée du travail et la flexibilité sont désormais négociées ensemble et non distinctement: " il faut que la réduction du temps de travail soit une réponse alternative à la flexibilité ».

L'idée d'un partage du travail reste à l'ordre du jour, même si l'appauvrissement relatif des salariés apparaît comme un obstacle à la perte de salaire : "C'est la solidarité dans une situation désastreuse. Mais je crois aussi que c'est peut-être plus difficile d'être solidaire au jour d'aujourd'hui qu'à une époque où il était facile de partager quand on en avait beaucoup. C'est vrai que maintenant, on nous dit de temps en temps : vous voulez qu'on partage la misère? ».

La réduction de la durée du travail semble difficilement réalisable aux responsables de la CFDT parce que les mentalités ne semblent pas encore prêtes à mesurer le travail par le temps et qu'il semble difficile de se retirer momentanément de la vie professionnelle (congé de maternité, travail à temps partiel, formation, etc.) : «Il suffit de discuter avec les femmes qui partent en congé de maternité : avant, elles ont des remontrances, des remarques, pas forcément directes mais c'est des sous-entendus et après cela, si elles ont le malheur de prendre du temps partiel... la responsabilité..., c'est fini..., on est toujours dans ce schéma-là ».

La compensation de la réduction de la durée du travail peut être partielle, donc la perte salariale peut être envisagée, ce qui caractérise précisément l'idée de partage de travail. En revanche, les bas salaires ne devraient pas être touchés par cette perte: «J'ai reçu l'historique de la réduction du temps de travail et c'est vrai qu'il y a une dizaine d'années dans un des congrès CFDT un amendement était passé dans un texte du congrès disant que la réduction du temps de travail jusqu'à 2 fois le SMIC était compensé \{sans perte salariale\} et c'est quand même quelque chose qui est un peu resté ».

Ce plafonnement de la perte salariale a été utilisé dans les négociations de branche en 1996 : «C'est ce qui a été dit aux négociations CFDT des branches ».

La CFDT accepte l'annualisation du temps de travail si elle est accompagnée d'une réduction de la durée du travail : " Plus c'est flexible, plus il faut réduire », et c'est dans ce sens que les négociateurs de branches CFDT ont négocié sur le temps de travail : «Tous les négociateurs de branches ont été réunis 3 jours, surtout sur l'annualisation puisqu'on sentait déjà que ça allait être ça. Donc, pour pouvoir commencer les négociations, le principe a été : plus c'est flexible, plus il y aura de la réduction du temps de travail. Il fallait se préfixer des grilles qui les amènent à avoir la mécanique dans la tête ». 
C'est la question de l'emploi des jeunes en particulier qui motive cette politique de la réduction de la durée du travail : «Il faut quand même savoir que sur les accords un des objectifs des organisations syndicales et des partenaires sociaux en général, c'est un message fort envers le gouvernement en lui disant ce qu'il n'a pas été capable de faire. Nous, on s'investit pour essayer de régler le problème de l'emploi des jeunes notamment à travers ces accords $»$.

«C'est bien pour l'emploi, pour créer des emplois et des emplois pour des jeunes puisque nous à la CFDT dans nos revendications on dit : 1 embauché sur 2 est un jeune ».

Selon la CFDT, on est passé d'un mode de réduction du temps qui voulait réduire la pénibilité du travail au début du siècle, à un mode de réduction individuelle portant sur de nouveaux arbitrages vie au travail/vie familiale. La perte de la référence hebdomadaire est ici considérée comme un défi et une opportunité pour construire de nouveaux arbitrages : « Au début du siècle, on a parlé de réduction du temps de travail mais il y avait quelque chose à prendre en compte, c'est que le travail était difficile. Il y a toujours du travail difficile, mais il y a une grande partie des salariés qui ont maintenant un travail à peu près correct physiquement. Donc, jusqu'à un avenir très proche, les salariés avaient négocié la réduction du temps de travail pour diminuer la pénibilité du travail et là c'était général. Actuellement, on est aussi sur un mode individuel, individualiste même et derrière ça la question est : comment lier mieux la vie professionnelle et la vie familiale ? C'est vrai que c'est une approche individuelle».

Pour régler la question de l'emploi et réguler la flexibilité, la CFDT fait plus confiance à la négociation collective qu'à l'État et à la loi puisque les salariés et les organisations syndicales ont du mal à s'opposer à une loi.

« La différence entre une loi et un accord-cadre est que derrière la loi, on a beaucoup plus de difficultés pour canaliser les travers. Tandis qu'avec un accord-cadre, disons qu'il a été signé au niveau des patrons et des organisations syndicales, quand on descend jusqu'à l'entreprise, il y a quand même des canaux de chaque côté qui permettent d'ajuster en permanence. Une loi, elle arrive, les patrons y font référence tout de suite et puis les salariés ont beaucoup de difficultés à aller contre, surtout dans des domaines comme la flexibilité qui était faite pour eux $»$.

Le travail de nuit des femmes continue à être approuvé par la CFDT en termes d'égalité des sexes et des carrières, mais il doit être soumis au volontariat : «Certaines disaient que ça allait pénaliser leur carrière, d'autres disaient que ça existait déjà et que c'était l'anarchie, donc réglementons un peu tout ça pour avoir des compensations. Donc, c'est toujours la même position ». 
Un problème actuel de la négociation du temps de travail, soulevé par un responsable de la CFDT, concerne la distance entre l'entreprise et la branche notamment parce que les signatures d'accords locaux ne sont pas toujours dans l'esprit de l'accord de branche. L'union régionale CFDT tente alors d'intégrer les militants des entreprises dans le fonctionnement interprofessionnel. Le désert syndical des PME, PMI est particulièrement considéré comme un frein au mouvement de négociation d'où la signature de l'accord interprofessionnel sur la possibilité d'une délégation unique : «On a signé l'accord sur la politique contractuelle dans les PME et les PMI parce qu'on s'aperçoit que c'est le vide pour ne pas dire le désert syndical. Il était question dans cet accord de mentionner que quelqu'un qui ne soit pas d'un syndicat puisse négocier un accord d'entreprise. Par contre, on met en place un comité de vigilance dans la branche où là, il y aura des organisations syndicales et c'est ce comité de vigilance qui validera l'accord d'entreprise, ceci pour permettre quand même une représentation des salariés dans les PME-PMI ».

Le profil de la négociation que les responsables de cette organisation se donne dans la négociation du temps de travail vise donc bien à maintenir l'idée d'un partage du travail en limitant la perte salariale aux salaires élevés. L'autre volet de sa politique du temps de travail concerne le contrôle de la flexibilité par la négociation en vue notamment de structurer les termes de l'échange avec la réduction de la durée du travail.

\section{II.2.B. La CFTC : solidarité et protection de la famille}

Les mots-clefs caractérisant cette organisation syndicale sont : « solidarité et famille ». «L'idée essentielle de ce syndicat, c'est la défense de la famille, les valeurs de la famille ».

C'est le fonctionnement de la cellule familiale qui est au centre de la politique syndicale et qui se traduit notamment par la gestion des allocations familiales. On met en avant une conception du travail qui n'est pas un but en soi, mais qui doit permettre une vie familiale « correcte » : «Il y a des cas où les parents se croisent et ne se rencontrent jamais, les enfants sont livrés complètement à eux-mêmes, et il y en a de plus en plus, alors, parallèlement à ça, on s'aperçoit que les gens n'assument plus leurs responsabilités familiales, ils n'ont plus la conscience des responsabilités qu'ils prennent. Typiquement, aujourd'hui, les gens ne sont pas tournés vers leur famille,... on veut faire leur bonheur malgré eux ».

A la CFTC, on envisage toujours une réduction de la durée du travail et une réduction des heures supplémentaires comme début d'un meilleur partage du travail : «Moi, je vous avoue, c'est plus un problème à expliquer aux employeurs. Pourquoi font-ils faire des 
heures supplémentaires, pourquoi n'embauchent-ils pas ? Je sais ce qu'ils répondront. Je trouve illogique qu'ils fassent faire des heures supplémentaires. Quand on parle de partage du temps de travail, il faut le partager là, c'est ridicule de faire des heures supplémentaires. Mais je vous avoue que les salariés sont pour les heures supplémentaires. S’il n'y avait pas d'heures supplémentaires, ça ferait des emplois en plus. Ce n'est pas mathématique ».

Le partage du travail s'impose comme une nécessité de solidarité incluant de toute manière une perte salariale : «Je pense qu'on ne peut pas faire autrement, je crois qu'on est obligé de partager, il y a de moins en moins de travail, on sera bien obligé de partager la pénurie à un certain moment, ce n'est pas une bonne chose, mais comment peut-on faire autrement ? Il faut arriver à baisser le nombre d'heures de travail, il faudra bien aussi que les salariés acceptent une baisse de leurs salaires pour qu'il y ait ce partage ».

Du point de vue de l'organisation du temps de travail, le travail de week-end, et en particulier le travail du dimanche, est rejeté en raison de la désynchronisation avec les activités familiales. Le responsable cite le cas d'un accord où les salariés choisissent le travail de week-end plutôt que le travail de nuit contre les activités familiales : «Pour un accord d'aménagement du temps de travail, le cycle de travail qui avait été mis en place, prévoyant du travail le dimanche, c'était du travail de week-end plus exactement, 1 dimanche sur 4 dans l'année et 1 samedi sur 2, donc c'est relativement lourd. La direction avait demandé l'avis des gens, s'ils préféraient travailler de nuit ou s'ils préféraient travailler le week-end, ils ont préféré travailler le week-end. Soyons clair : la nuit, on voit pas ses enfants, les gens ne pensent qu'à leurs loisirs, ce qu'ils voient c'est qu'ils vont pouvoir dégager du temps dans la semaine, ils vont pouvoir faire leur petit sport, etc.. L'état d'esprit global des gens malheureusement est d'un égoïsme assez forcené, donc l'aspect familial, ils le passent à la trappe complètement et, malgré eux, si je puis dire, on tiendra bon, on essaiera de faire en sorte qu'ils voient leur famille ».

Le travail de nuit est à limiter si possible pour arranger la vie familiale, mais la modulation-annualisation est envisageable dans certaines limites : «Les nuits ne favorisent pas la vie de famille et nous ne sommes pas opposés comme ça, il y a des endroits où on ne peut pas faire autrement, mais notre approche n'est quand même pas de généraliser ce type de travail. Nous sommes plutôt pour une modulation du temps de travail le reste de la semaine mais pas le week-end $»$.

Le travail de nuit des femmes est fermement rejeté : «Nous avons toujours été contre formellement sans discussion possible. Toutes nos positions confédérales sont claires et nettes. Nous sommes contre, parce que c'est une conception de la famille qui va à l'encontre de nos idées, de notre éthique ». 
En revanche, le temps partiel est bien accueilli notamment pour les couples et les familles : «Je pense que le travail à temps partiel pour certaines cellules familiales est une bonne chose. Je pense que les salaires tels qu'ils sont, pour une seule personne qui travaille ne sont pas suffisants, deux, c'est souvent un peu trop, mais un et demi, c'est souvent parfait, ça permet, de faire un complément, de vivre plus correctement, et ça permet aussi à la personne de garder toujours le contact avec le monde du travail, de ne pas perdre le contact avec l'extérieur, c'est toujours intéressant et en même temps de s'occuper de sa famille aussi, c'est une bonne chose. Je pense que pour certains couples ce serait bien ».

Selon la CFTC, la négociation est en crise actuellement : «Le syndicalisme s'est enfermé un certain moment parce que les gens ont peur de s'engager, de prendre une carte syndicale $\gg$.

Dans l'avenir, l'harmonisation des politiques syndicales en France est considérée comme difficile en raison des différences culturelles, intellectuelles et morales : «A tous les niveaux, même au niveau de la CFTC, l'approche d'un Alsacien et l'approche de quelqu'un de Bordeaux n'est pas la même. Je pense que ça vient de la conception des gens, intellectuellement, moralement. Faire cette harmonisation est difficile, pourtant on vit ensemble depuis fort longtemps ».

En revanche, l'unité et la solidarité notamment en matière de négociation du temps de travail doivent rester l'objectif : «Pour la négociation sur le temps de travail à la CFTC, je pense que la solidarité, comme dans tous les syndicats est un maître-mot. Disons que c'est une approche qui est là en même temps chez toutes les organisations syndicales. C'est l'économique qui gère, qui nous oblige à revoir nos positions anciennes. C'est vrai que la réduction de la durée du travail, la modulation sont à l'ordre du jour et on ne peut pas faire autrement. A la CFTC, on pense tous que c'est une approche essentielle. On ne peut pas faire autrement que réduire le temps de travail et faire un meilleur partage du travail ».

\section{II.2.C. La CGC : la semaine de 4 jours et l'acceptation de la flexibilité}

Les mots-clefs de l'organisation régionale sont l'amélioration des conditions du travail et la protection de la vie économique et industrielle de l'entreprise : «C'est améliorer le travail des salariés tout en préservant la vie économique et industrielle de l'entreprise. Il faut arriver à un bon amalgame des deux entités, des intérêts du salarié et de l'entreprise. Il est hors de question de détruire l'un des deux. Cela détruirait l'autre ».

Les accords sur la réduction de la durée du travail qui ont pour but de créer de l'emploi sont considérés comme inefficace et même comme destructeur d'emploi, car ils augmentent souvent la productivité de l'entreprise : «La CGC est pour la réduction de la 
durée du travail avec un intérêt derrière, un intérêt d'embauche. Dans $90 \%$ des cas, j'ai l'impression que c'est surtout pour préserver de l'emploi, c'est un peu du chantage à l'emploi en disant: nous mettons cet accord en place, ça évitera X licenciements. C'est pour ça qu'on observe à mon avis un pourcentage élevé de signatures, mais quand on regarde avec du recul, si on reprend ces accords 3 ou 4 ans après, on s'aperçoit que momentanément, ça a peut être servi à quelque chose, mais ça n'a rien résolu et il y a quand même des licenciements au bout d'un certain temps. Donc, c'est ça qui me gêne dans tous ces accords, c'est qu'on attend de polluer le système, de ne pas créer d'emplois et au contraire, il y a des pertes d'emploi au bout».

L'idée du partage du travail exprimée par la CFDT et la CFTC est rejetée en particulier à cause de la perte de salaire et par conséquent de la perte pour les cotisations sociales mettant en danger les plus démunis : «Si vous avez $100 \mathrm{~F}$ et qu'on les partage en 2 fois $50 \mathrm{~F}$, ça ne servira à rien. Tant qu'on n'aura pas créé des emplois avec un système quelconque. Je ne vois pas un système qui va la mettre en place et là ça devient grave. Je ne vois pas ce qui peut marcher, parce qu'à force de partager la misère, la pauvreté, si tout le monde est pauvre, il n'y a aucun intérêt. On peut critiquer les différences de salaires, mais tant qu'il y a des salaires élevés qui permettent de payer des cotisations pour avoir des aides à un certain niveau, ça marche. Le jour où tout le monde est à un niveau inférieur et que plus personne ne peut payer même pour ceux qui sont plus bas, là il va y avoir de sacrés problèmes ».

Ainsi, la confédération des cadres préconise le passage à la semaine de 4 jours sans parler de réduction d'heures de travail. Il s'agit de diminuer la durée du travail effectivement en ne travaillant que 4 jours sur 7 jours. C'est la formule qui a été retenue pour défendre la population des cadres travaillant au forfait: "C'est que l'on demande la semaine de 4 jours, mais semaine de 4 jours, comme étant 4 fois 10 heures. On fait les horaires hebdomadaires en 4 jours, les gens ne pourront pas faire 4 fois $12 \mathrm{~h}$, c'est clair, ça serait plafonné systématiquement à la semaine de $40 \mathrm{~h}$ Aujourd'hui, tous syndicats confondus, je crois qu'il y a une prise de conscience très élargie à ce niveau-là sur ce qu'on peut faire pour limiter le temps de travail des salariés forfaitaires ».

Les durées longues de travail des cadres sont en cause : «On approche plus facilement les $50 \mathrm{~h}$ par semaine qu'autre chose, donc c'est pour ça que j'étais descendu à 40 h. Au moins, c'est clair, c'est net, c'est propre, personne n'est frustré, on sait pourquoi il y a une perte de 10 heures dans ce cas-là. C'est pourquoi ça entraîne une perte de salaire, c'est facile, c'est clair, c'est quantifiable ». 
" Si on dit qu'on veut descendre à 32 heures ou à 35 heures peu importe le chiffre et qu'on reste sur l'aménagement du temps de travail actuel, ça ne changera rien, ça amènera à baisser peut être d'une demi-heure par jour. Pour quelqu'un qui est cadre, il est clair qu'une demi-heure par jour, on la fera quand même. On ne fera pas plus d'heures, on fera toujours les mêmes heures, mais il est évident qu'on aura une diminution de salaire. Donc nous, on défend la semaine de 4 jours, c'est peut-être 4 fois 10 heures, je ne sais pas, c'est suivant l'entreprise en disant : on est là présent 4 jours, mais au moins le 5e jour, on est vraiment de repos, on est chez nous ».

Pour la flexibilité qui pourra constituer une contrepartie d'une réduction des heures, ce sont également les conditions locales qui détermineront son ampleur : "Pourquoi on ne fixe pas des heures souples? Parce que ça ne peut être négocié que par l'entreprise. Ce qui se passe aujourd'hui le démontre très bien »"

Le principe du travail de week-end et du travail posté pour les cadres est accepté et dans les faits il est déjà pratiqué localement. Il pourrait être échangé dans la mesure où la semaine de 4 jours pourra être réalisée : «C'est vraiment 4 jours. C'est-à-dire que la même personne est maintenant là 4 jours. Mais ça peut être 3 jours, un vendredi, samedi, dimanche. Ce qui équivaut à 4 jours avec les difficultés du travail du dimanche et ensuite, on a la possibilité de tourner. Il y a toujours des mixages possibles à 3 équipes. Peut-être 2 équipes aussi qui se recoupent [...]. Vous avez des machines aujourd'hui, des ordinateurs. L'intérêt d'avoir un ordinateur, c'est qu'il fonctionne 24 heures sur $24 »$.

La CGC considère que la vie hors travail et la vie familiale peuvent faire l'objet d'une meilleure organisation en intégrant le week-end au travail habituel : «J'ai travaillé pendant longtemps dans les transports aériens. J'ai travaillé les jours fériés, les dimanches, des heures croisées dans tous les sens. Cela pose des difficultés mais il y a des avantages. Quand vous avez toute une matinée de libre ou toute une après-midi, vous travaillez un week-end, c'est vrai, mais en contrepartie dans la semaine vous avez 2 jours où si vous pouvez bricoler, visiter, vous êtes avec vos enfants, votre femme. C'est une autre organisation, vous pouvez profiter d'un certain nombre de choses que vous ne pouvez pas faire le week-end, car le dimanche tout est fermé et le samedi tout est ouvert, mais il y a un monde fou $\gg$.

Le travail de nuit est approuvé aussi pour les femmes à condition qu'il s'agisse d'un libre choix ce dont doutent les responsables dans certains cas : « De fait il existe, quand vous regardez les hôpitaux, la restauration où les femmes travaillent de nuit, finissent souvent à minuit aussi bien en restauration qu'en service. Alors, on retombe sur le même problème qu'on évoquait pour le travail du dimanche. Je ne suis pas fermé à ça, bien au contraire. Le 
seul problème c'est qu'est ce qui me prouve que c'est voulu, qu'on n'est pas en train de faire pression sur la personne pour qu'elle reste la nuit ou pour qu'elle vienne le weekend? Le problème le plus important c'est celui là : avoir la liberté de choix de son travail, de la manière de travailler. Si on est sûr de ça, moi ça ne me gène pas. Il y a du personnel féminin qui préfère faire ça \{travailler la nuit\} parce que dans la journée elle peut aussi s'occuper de ses enfants, si elle en a, elle peut faire autre chose. Si c'est voulu, si ça lui plaît, il n'y a aucune raison de lui interdire. Je suis pour la liberté de choix. Le problème est que ce ne soit pas imposé. Le travail de nuit des femmes peut être aussi un avantage si c'est voulu et bien suivi, d'où l'importance dans ces milieux-là des organisations syndicales qui puissent suivre l'évolution de ce travail ».

L'annualisation est acceptée en particulier, parce qu'elle évite la propagation de contrats à durée déterminée et des licenciements : «Je pense que c'est assez intéressant et que ça évite les licenciements ou des CDD, ça fixe un peu plus le personnel et ça peut le motiver justement. On n'a aucun intérêt en période de charge à embaucher du monde pour les licencier 2 mois plus tard parce qu'il n'y a plus de boulot pendant 3 mois ».

Le travail à temps partiel est également soutenu à condition qu'il soit volontaire : «Le travail à temps partiel, je pense qu'il faut que ça soit inscrit dans les accords d'entreprise mais que ce soit un temps partiel voulu et non imposé, c'est le premier point. Le second point est que ce ne soit pas un frein à la carrière ; on doit avoir un suivi de la carrière de ces gens-là. Si on l'a négocié, l'entreprise était d'accord pour le mettre en place, donc il faut le suivre. Donc on est favorable au temps partiel ».

Les heures supplémentaires sont jugées trop nombreuses, mais on constate une volonté des salariés d'en effectuer. Ce qui place l'organisation entre le souhait des salarié et la défense de l'emploi : «c'est tout à fait normal que plutôt que de faire des heures supplémentaires à gogo, on puisse diminuer voire faire disparaitre ces heures pour faire des embauches. Mais ça pose un autre problème : si les syndicats sont favorables à ça, êtes-vous sûr que les salariés concernés sont favorables? Du jour au lendemain, on les enlève, vous ne les faites plus, le gars va regarder sa feuille de paie. Dans quelle logique on se place ? Défense du salarié qui existe ou défense de l'emploi ?».

Un des axes de travail au niveau national concerne la mise en place d'un statut du militant syndical, car on constate une hostilité du travail syndical au niveau des directions : «Qu'on puisse mettre en place un statut du militant syndical, qu'il soit vraiment reconnu en tant que tel, qu'il puisse exercer en toute liberté, c'est-à-dire sans pression, pas faire n'importe quoi, ce n'est pas dans ce sens là, mais exercer en toute liberté son mandat et 
qu'il ne subisse pas du fait qu'il fait du syndicalisme une certaine ségrégation de la part de sa direction, j'ai plein d'exemples ».

\section{II.2.D. La CGT : plein-emploi et stabilité des repères temporels}

Les mots-clefs de l'organisation régionale sont ceux qui décrivent le début du syndicalisme: «Le syndicalisme en général s'est créé sur des idées de fraternité, de solidarité et de justice. Au niveau des valeurs c'est toujours les mêmes ».

La réduction de la durée du travail fait partie des revendications pour améliorer la vie du salarié. Le temps de repos est du temps pour vivre, pour les loisirs, mais aussi du temps pour créer des emplois. Le principe d'une réduction de la durée du travail est adoptée sur une base de compensation intégrale de la perte salariale, voire une augmentation du salaire global : «Aujourd'hui, on milite pour une réduction effective du temps de travail, mais qui s'accompagne des salaires qui puissent permettre de profiter de ce temps libre. Si c'est pour aller s'enfermer dans la maison et ne pas avoir d'argent pour acheter un livre, ne pas avoir de moyens pour sortir, ne pas avoir accès aux loisirs, ça poserait des problèmes, mais avec un pouvoir d'achat qui serait suffisant, de manière à avoir accès à la culture, à la peinture et à la musique. Gagner du temps pour vivre, pour être en famille ».

La réduction de la durée du travail devrait être financée par les gains de productivité, donc par une partie des profits accumulés par les propriétaires des moyens de production : «On se bat contre un système, parce qu'il y a une masse importante de profits justement et avec cet argent, on a de quoi financer la réduction de la durée du travail. Il y a de l'argent, donc c'est possible, mais il va falloir se le gagner. Cela me parait possible, quand on regarde l'évolution des profits, quand on regarde les masses de production qu'on arrive à faire et quand on regarde où est la productivité ».

La réduction de la durée du travail avec maintien du salaire s'oppose au chômage et à la productivité. La revendication principale concerne alors une loi-cadre sur l'instauration de la semaine des 35 heures votée par l'Assemblée Nationale : «Nous, on dit qu'il faut un accord-cadre de 35 heures par semaine et que ce soit voté à l'Assemblée Nationale, que ce soit le fait de la législation de manière que ce soit applicable à tout le monde et on n'accepte pas qu'on nous dise qu'on nous le renvoie par branche, par la négociation par entreprise. Chaque fois qu'il y a eu une réunion sur le temps de travail, ça s'est fait dans le cadre législatif. Donc nous, on demande à ce que cette fois-ci, ça se fasse comme ça aussi et en même temps on demande à ce qu'il n'y ait pas de perte de pouvoir d'achat ».

La perte salariale dans les arrangements sur le temps de travail est rejetée par principe et le législateur est invoqué pour définir des durées du travail plus réduites. L'objectif à plus 
long terme concerne la semaine de 4 jours (32 heures) qui pourrait impliquer aussi les cadres du fait que la réduction en nombre de jours les intéresse: "L'objectif visé aujourd'hui c'est bien 32 heures, la semaine de 4 jours, c'est ça. Alors qu'avant on était contre syndicalement. On est pour l'essayer et aujourd'hui ce qui monte dans les réunions qu'on fait avec les uns et les autres, c'est les 4 jours. L'idée est de dire que si on fait 32 heures, on sera toujours marron parce qu'on fera plus de 32 h en réalité. Par exemple pour le cadre, si on lui dit : tu as 32 heures, ça ne va pas lui changer grand chose au niveau des heures de travail, mais si on le met sur 4 jours, forcément si ».

La flexibilité du temps de travail dont le symbole est l'annualisation, est rejetée parce que 1) elle réduit la masse salariale (par le non-paiement des heures supplémentaires), 2) elle supprime les intérimaires et les contrats à durée déterminée et 3) elle est l'instrument du patronat pour empêcher les revendications syndicales sur la réduction de la durée du travail et pour réduire le pouvoir syndical : «L'annualisation du temps de travail, on n'est pas du tout d'accord avec ce système-là, c'est le système pour arriver à diminuer le temps de travail effectif, donc peser sur la masse salariale et de l'autre côté, c'est le moyen de ne pas créer d'emplois du tout parce que dans une entreprise aujourd'hui, vous avez un effectif qui est un effectif moyen, on l'a embauché pour un plan de charges et en fonction de l'activité de l'entreprise, vous avez des périodes où on prend des CDD, ce n'est pas des bons emplois, mais c'est de l'emploi quand même, les CDD, les intérims, tout ça, et globalement dans l'année vous avez une masse de salariés. Avec le système de l'annualisation du temps de travail, vous n'aurez plus recours à l'intérim, aux CDD. Quand il n'y aura pas beaucoup de travail, les gens, on les fera rester à la maison. En fait, c'est les salariés qui sont en fixe dans l'entreprise qui vont devenir complètement flexibles et qui vont faire les hauts de crêtes, les bas de crêtes au niveau de la production, ce qui évitera à l'employeur d'avoir recours à la main d'œuvre extérieure quand il est en haut de crêtes, ça va lui permettre de jouer contre l'emploi. Concrètement, c'est l'expérience que j'avais dans l'entreprise $»$.

L'échange aménagement ou annualisation contre réduction de la durée du travail est strictement refusé. Il s'agit pour la CGT d'une stratégie de prise d'initiative patronale sur le terrain classique de la revendication syndicale pour contrôler la direction des négociations. La société est en train de devenir une société à temps partiel (imposé) en particulier par la propagation des contrats emploi solidarité (CES). Ceci renvoie alors au fonctionnement du système global qui est à combattre : «Est ce qu'on va en faire tous des vacataires, des CES, une société à temps partiel parce que, quand j'ai commencé à travailler, ça n'existait pas du tout ça : 40 heures par semaine voire 50 et puis il n'y avait 
rien d'autre, c'était rare quand il y avait autre chose. Après sont arrivés les travailleurs intérimaires. Comme les intérimaires étaient bien payés, ça permettait de rouler sa bosse, de connaître le travail, puis on s'est retrouvé avec des intérimaires qui ne choisissaient plus rien. Maintenant, quand vous voyez encore des gens qui sont à $39 \mathrm{~h}$ par semaine, mais c'est des anciens. Cela commence à devenir inodore ».

La CGT veut mener une politique à long terme qui freine l'ouverture des plages-horaires qu'elle considère comme socialement réservée au repos (soir, nuit, week-end). En particulier, le travail de nuit des femmes, bien que compréhensible du point de vue de certaines femmes, ne peut pas être approuvé comme un élément de la politique syndicale du temps de travail : «Les difficultés de la vie quotidienne font qu'à un moment donné les gens sont obligés d'accepter n'importe quoi. Une femme ne souhaite pas travailler la nuit, mais si elle avait le choix de travailler la nuit ou pas, ou si c'était un frein à son déroulement de carrière parce que là où elle est, elle ne peut pas faire le même travail qu'un homme et qu'elle voit que, finalement, elle n'aura pas la promotion qui va avec. Si je me mets dans la peau du personnage, c'est elle qui a raison et on ne peut pas se mettre dans la peau de chaque personnage parce que chacun à son niveau a ses raisons. Il vaut mieux avoir une ligne politique. On n'a pas toujours raison sûrement. En principe, on pense qu'on a raison d'essayer d'avancer dans ce sens là. Si on s'aperçoit qu'on s'est trompé, on rectifie le tir, mais sinon, faire forcément ce que les gens pensent être le mieux, on ne peut pas être d'accord tout le temps avec les gens ».

Dans la situation actuelle, l'action syndicale a pour but de limiter l'offensive patronale, notamment en matière de temps de travail : «La seule limite qu'il aura \{le patronat français\} sera des limites qu'on sera capable de lui mettre devant, c'est une machine qui va avancer, on ne sait même pas jusqu'où elle peut aller. Il n'y a personne aujourd'hui qui peut dire comment seront les conditions de travail ou la vie de la société française. Moi, si on m'avait dit il y a 25 ans, qu'aujourd'hui on serait comme on est, j'aurais rigolé ».

\section{II.2.E. FO : Contractualisation et résistance à la guerre économique}

Contrairement à l'image donnée de FO dans les médias : « une organisation syndicale qui se radicalise et qui n'accepte pas la politique contractuelle », FO défend avant tout une politique contractuelle avec le patronat: «Nous sommes l'organisation qui signe le plus d'accords parce que nous préférons un accord à une imposition. Un accord, c'est quoi ? Lorsqu'on contracte quelque chose, c'est un compromis ».

Les mots clefs caractérisant l'organisation syndicale au niveau régional sont «discuter, négocier et contracter $»$. 
La réduction de la durée du travail est considérée comme un fait socio-historique impulsé par la lutte syndicale que FO pense mener sur l'augmentation des congés et la diminution de la durée hebdomadaire du travail pour améliorer la vie des salariés : «Depuis déjà quelques mois, nous revendiquons la 6e semaine de congés payés puisque effectivement, c'est la revendication forte d'aujourd'hui. Parallèlement à cela, nous considérons qu'il doit y avoir une réduction de la durée hebdomadaire du travail. Alors, nous pouvons avoir plusieurs approches. On peut avoir une approche proprement revendicative qui dit: pourquoi pas 30 heures. Pourquoi pas 25 heures? Nous restons quand même persuadés qu'aujourd'hui, il faut une réduction réelle, importante, du temps de travail, tout simplement en contrepartie sociale $»$.

La revendication principale concerne une réduction de la durée du travail interprétée comme une contrepartie sociale de l'évolution de l'activité économique. En revanche, la question du chômage ne pourra pas être résolue par la réduction de la durée du travail. Les expériences menées dans différents secteurs montrent au contraire qu'un partage du travail qui est considéré avant tout comme un partage des salaires est inefficace, car résorbé automatiquement par l'augmentation de la productivité : «Aujourd'hui, sur la réduction de la durée du travail, on est quand même très limité, parce qu'on a vécu un certain nombre d'expériences. Nous avons été signataires d'un certain nombre d'expériences et je dis qu'effectivement on ne pourra pas tout traiter par l'économique et qu'on sera bien obligé aussi de traiter par le social. Alors, il faut savoir ce que l'on veut et la dimension qu'on veut mettre. Sauf que les entreprises vont vous dire : pour faire du social, il faut que j'aie de la production, il faut que j'aie des gains de productivité qui me permettent de faire du social ».

Les heures supplémentaires sont jugées trop importantes (130 heures annuelles) et FO propose une limitation annuelle à 90 heures de celles-ci : "C'est déjà un bon matelas, 90 heures. Cela veut dire qu'on permet quand même à une entreprise en tant que telle, si elle a un coup de bourre à donner, un travail à faire, de passer une crête, comme on dit si bien ».

La flexibilité du temps de travail est incarnée par l'annualisation qui a pour but de désynchroniser les salariés: «L'annualisation, c'est ça. Ce qu'on veut, c'est des contrats atypiques. Quand vous avez cassé au salarié, sa façon d'exister, il n'existe plus. Il sait que le lundi, il travaille, le mardi, il ne sait pas, le mercredi encore moins et peut être le jeudi. C'est une stratégie antisyndicale formidable. A partir du moment où vous avez tous les gens qui ne sont pas là le même jour, qui ne travaillent pas à la même période, vous n'avez plus la possibilité de les regrouper. C'est ça que visent les patrons ». 
La négociation sur la flexibilité peut porter sur l'amplitude du travail journalier, mais on souhaite garder une structure de la société et de la vie sociale. L'absence de références communes menace en permanence l'existence de la société. Dans ce contexte, le travail de nuit des femmes est considéré comme une régression et non comme un progrès par rapport à l'égalité professionnelle entre les sexes : «D'abord, il faut arrêter de considérer, parce qu'elle est exploitée au même titre que les hommes, elle a gagné des galons, l'égalité. N'oubliez quand même pas que malgré tout, là aussi il va falloir arrêter. Je reviens toujours en permanence là-dessus, à ce que l'économique ne détruise pas tout. Vous croyez que des gamins qui rentrent de l'école ont exactement les mêmes chances, y compris de réussite. Le gamin qui, quand il rentre de l'école, ne trouve pas le cocon familial».

Pour le travail à temps partiel, le jugement est plus nuancé : «Sur le temps partiel, on a signé des accords dans l'entreprise, c'est-à-dire qu'on a souhaité que les salariés hommes ou femmes d'ailleurs, il faut arrêter de faire la distinction, de considérer qu'il n'y a que les femmes qui peuvent demander un temps partiel, ça peut être aussi des hommes. Il faut quand même que vous sachiez que contrairement à ce que l'on dit, même si la panoplie existe, lorsqu'on veut l'appliquer dans l'entreprise, ce n'est pas aussi évident que ça. Il nous arrive très souvent de nous battre pour demander effectivement à ce que l'on puisse accorder un temps partiel à tel ou tel agent parce que ce n'est pas toujours évident, parce que ça n'arrange pas toujours l'entreprise, parce que des fois, par cascade, ils sont obligés de créer un poste et ils n'y tiennent pas tant qu'effectivement cette réduction est absorbée. Il faut savoir qu'à 70, $75 \%$, quand il y a du temps partiel, c'est résorbé tout simplement par l'environnement de l'agent qui est à temps partiel ».

La situation défavorable des syndicats et l'adoption d'un syndicalisme de défense s'expliquent par le chantage qui leur est fait sur la question de l'emploi et aussi par l'écart entre le référentiel négociatoire local et national: «On a eu des accords avec une généralisation des $3 \times 8$, des entreprises qui tournaient d'une façon permanente, avec à la clef une réduction de la durée du travail, incontestablement aussi des femmes qui travaillaient la nuit et qui se retrouvaient avec des décalages très importants. Le chantage qui a été fait était le suivant : ou vous acceptez l'accord ou c'est le chômage. Comme l'outil de production est capable d'absorber un certain nombre de gains de productivité, aujourd'hui le patronat est très à l'aise pour effectivement dicter un petit peu sa loi ».

C'est ainsi que les salariés acceptent le travail de nuit et de week-end sous la contrainte économique : «Vous savez, tout se traduit en terme économique. Ce qui est bien la démonstration que si les gens gagnaient suffisamment pour s'éviter la prime de nuit et de week-end, ils ne feraient pas ». 
De manière générale, on observe un renforcement de la résistance des salariés contre la politique du patronat et on s'attend, comme par le passé, à une régulation cyclique par des crises sociales plus ou moins aiguës. Le rôle du syndicat est de résister contre la déréglementation en cours et contre la guerre économique : «On est en train de nous conduire à une déréglementation totale, une déréglementation qui n'a pas de but. Jusqu'à l'implosion du système. Autrement dit, ce qui nous amène (je vais être un peu méchant, je vais presque les comparer aux mages du temple solaire) au suicide collectif. Lorsqu'on parle de compétitivité, lorsqu'on nous dit: il faut qu'on fasse ça parce qu'on va être meilleur, quelque part c'est prendre celui qu'on a en face pour un imbécile parce que, si vous, vous êtes capable d'avoir ce raisonnement, ça veut dire que l'autre en face, il n'est pas capable de faire comme vous et c'est quoi ? C'est l'escalade, c'est comme la guerre tout simplement, là, c'est la guerre économique ».

\section{II.2.F. Conclusion}

Notons que chaque organisation syndicale a ses préférences en matière de temps de travail et que le référentiel temporel est spécifique d'une organisation à l'autre. Nous pouvons extraire alors la différence des positions (thème par thème) ce qui nous donne du point de vue d'une région un profil du syndicalisme français l'égard du temps de travail.

A travers l'analyse des positions par rapport aux thèmes du temps de travail, nous constatons que la convergence des positions dépend du thème considéré. En aucun cas, il ne semble y avoir une approbation unanime de la flexibilité ou du partage du travail. Si tout le monde se met d'accord sur le principe d'une réduction de la durée du travail, les organisations syndicales divergent sur les modalités. Dans la question de la flexibilité (notamment pour le temps partiel et le travail de week-end) réapparaît la question de la famille et de la division du travail entre hommes et femmes. Les uns et les autres mettent en avant leur conception de la vie hors-travail et du rapport des genres. Cette distinction des référentiels de négociation qui sont mobilisés, montre que l'unité de l'action syndicale par rapport au temps de travail reste à construire.

Au-delà des frontières organisationnelles, il nous semble qu'une attitude commune au moins sur certains principes extraits des entretiens pourrait nous renseigner sur la quête du syndicalisme français en matière de temps de travail. Mais il nous semble nécessaire de reposer les questions du temps de travail exclues des codifications par thèmes, car la logique de situation seule ne nous semble pas ici satisfaisante pour éclaircir l'approbation des accords. Si nous laissons de côté les profils propres de chaque organisation suivant les 
thèmes, nous obtenons de nos entretiens une trame qui explique, malgré les spécificités que l'acteur syndical semble bel et bien mettre en cause la fin d'un temps de travail.

\section{II.3. Les syndicats unis contre un ordre temporel exclusivement orienté vers la production}

Le référentiel négociatoire oriente les positions de chacun des syndicats en combinant des valeurs tournées soit vers la prise en compte des exigences liées à l'ordre de la production, soit vers la prise en compte des exigences liées à la vie quotidienne des salariés. Dans ce référentiel s'articulent des positions visant à combiner plusieurs temporalités, les unes tournées vers la vie professionnelle, les autres vers la vie non professionnelle.

Il reste à comprendre de quelle manière les singularités syndicales peuvent néanmoins contribuer à une action collective. Les spécificités dans les valeurs avancées par chaque syndicat constituent-elles un facteur de division ou de regroupement des syndicats ? Notre hypothèse est ici que le rôle du référentiel est double : d'un côté, il divise les syndicats, ou plus exactement les alliances entre syndicats sont locales et ne concernent pas tous les syndicats. Tel syndicat peut être d'accord avec l'autre sur la réduction de la durée du travail, mais s'opposer sur la question de l'annualisation. D'un autre côté, les syndicats de salariés s'entendent sur un certain nombre de valeurs constitutives d'un référentiel commun, basé sur un accord de fond sur certaines valeurs.. Ce référentiel commun est donc constitué par des valeurs de base telles que (a) avoir du temps pour soi et pour tous, (b) la défense de la stabilité des repères temporels pour favoriser la concordance des temps,

(c) la possibilité de contrôler son emploi du temps, c'est-à-dire de ne pas être assujetti aux exigences de la production et de ne pas être soumis à la simple volonté de l'employeur.

La méthodologie utilisée pour expliciter ces deux types de référentiels est la même que celle utilisée pour expliciter les référentiels propres à chaque syndicat.

Nous avons tenté d'extraire des entretiens les caractéristiques du référentiel commun. La pression temporelle (a), la stabilité des repères temporels (b) et la maîtrise du cadre temporel (c) constituent les principales caractéristiques de ce référentiel.

\section{II.3.A. Avoir du temps pour soi et du travail pour tous}

La première caractéristique du référentiel temporel de base est la revendication de disposer de «plus de temps pour soi». Bien entendu le thème n'est pas nouveau: Rezsohazy (1986) montre dans son enquête menée auprès de 1636 personnes en 1975 et en 1980 que ce qui gène le plus les gens dans ce qu'ils voudraient « faire ou être », c'est le manque de temps qui apparaît comme facteur le plus perturbant. De même, dans une enquête menée en 1982 (cité par Sue, 1994, p. 12) «le temps pour vivre» apparaît comme le premier 
centre de frustration des français. Ceux qui manquent de temps se retrouvent dans l'enquête menée en 1992 au Canada auprès de 9815 personnes (cité par Pronovost, 1996, p. 54) : la réponse la plus retenue porte sur le sentiment de « ne pas avoir tout accompli de ce qu'on voulait faire dans la journée ». C'est dans ce climat de pression temporelle que les organisations syndicales expriment leur point de vue qui peut se résumer par le propos de l'un d'entre eux : «plus de temps pour soi, du travail pour les autres ».

Cette expression se traduit, pour eux, par la nécessité d'une réduction de la durée du travail hebdomadaire, voire par la semaine de 4 jours pour avoir du temps pour soi.

« Il faut vraiment que la réduction soit forte, 35 heures, ce n'est peut-être pas suffisant, ça doit être une étape pour aller plus loin. L'objectif visé aujourd'hui c'est bien 32 heures, la semaine de 4 jours, c'est ça réduire le temps de travail, c'est gagner du temps de repos ; c'est du temps pour vivre, pour les loisirs pour être en famille » (CGT).

"Réduire l'importance ou l'amplitude du travail par un certain nombre de congés supplémentaires et parallèlement à cela, réduire aussi le temps de travail d'une façon hebdomadaire ou journalière et c'est ainsi que dans le processus, dans les revendications que nous avons mises en place, depuis longtemps nous avons affiché notre nécessité de revenir à $35 \mathrm{~h}$ par semaine » (FO).

« Je dirais que travailler 4 jours sur 7, ça laisse 3 jours de loisir » (CGC).

« Pour que la réduction du temps de travail soit efficace économiquement, il faut qu'il y ait 3 règles de mise en œuvre : (1) massive, l'objectif c'est d'atteindre les 32 heures par une loi-cadre, (2) multiple et (3) diversifiée pour tenir compte des différentes spécialités des entreprises » (CFDT).

« Ramener la semaine à $37 \mathrm{~h}$, pourquoi pas d'une manière très impérative, mais modulée » (CFTC).

En revanche, la question du partage du travail associée à une diminution de salaire divise les syndicats : trois d'entre eux pensent qu'elle est inéluctable. Pour les uns, la perte salariale est acceptée.

« Je dis : qui dit partage dit aussi diminution du salaire, il faut en arriver jusque là » (CFTC).

«Ce sur quoi on tourne sur la réduction du temps de travail, c'est le financement de la réduction du temps de travail et c'est là-dessus que d'ailleurs, on a des différends avec les autres organisations syndicales puisque les autres disent 32 heures sans réduction, sans perte de salaire. Nous, on est un peu plus nuancé » (CFDT).

Pour les autres cette perte salariale est rejetée. 
« Je pense que la réduction du salaire joue contre l'emploi parce que moins de salaire, c'est moins de consommation, moins de consommation, c'est moins de débouchés pour l'entreprise forcément à un moment donné, on est déjà dans cette difficulté, car ils ont tellement réduit les salaires de partout » (CGT).

«Quand on ne peut plus taxer les gens, c'est qu'ils sont à un tel niveau qu'ils ne peuvent plus. Voilà pourquoi, aujourd'hui, on ne peut pas établir une théorie de cette nature sans que pour autant, on regarde comment on l'applique » (FO).

\section{II.3.B. Stabilité des repères temporels et retour sur la famille}

La deuxième caractéristique du référentiel temporel concerne la revendication d'une stabilité des repères temporels. Les régularités temporelles qui scandent la vie d'un individu (école, travail, retraite) sont remises en cause, notamment par des périodes de chômage auxquelles sont soumis bon nombre de personnes, mais aussi par les situations de précarité qu'elles rencontrent au cours de la vie professionnelle. A cela s'ajoute la dissociation croissante entre, d'un côté, le temps des machines et, de l'autre, le temps des personnes insérées dans des activités multiples.

C'est dans ce climat de brouillage des repères temporels que s'expriment les positions des acteurs syndicaux à l'égard des mesures temporelles permettant l'annualisation du temps de travail, le travail à temps partiel, le travail de nuit des femmes. Une première illustration de cette revendication de stabilité des repères temporels nous est donnée par les réactions à l'égard de l'annualisation du temps de travail qui est considérée en référence à la famille et la division du travail qui s’y opère selon les sexes.

«L'annualisation, bien entendu, c'est un problème tout à fait différent et pour lequel on ne peut pas être d'accord, parce que l'annualisation est une question aussi de qualité de vie. L'annualisation veut dire simplement la destruction de la cellule familiale. Cela veut dire aujourd'hui qu'un chef d'entreprise va vous dire dans le cadre de l'annualisation : vous allez pouvoir travailler en fonction du travail que j'ai. Et ça peut déjà aller par rapport au congé parce qu'il n'est pas impossible qu'il y ait une commande pendant la période de congé et, comme il a de mai à octobre pour vous les donner (les congés), rien ne lui interdit de les donner en mai ou en octobre. Cela serait quand même pas inintéressant que le père prenne les vacances en octobre, que les enfants les prennent en juillet et août et que la mère les prenne au mois de mai » (FO).

«C'est la vie familiale qui est mise en cause. La mère de famille qui a des enfants ne pourra pas bloquer son temps de travail sur ce niveau de l'année et puis qu'est-ce qu'elle va faire les autres mois? Elle aura un temps de travail beaucoup plus long. Comment 
pourra-t-elle faire pour garder ses enfants ? Il y a un équilibre à respecter, je pense, il y a un cadre, mais ce cadre a des limites. Des limites à mon avis qu'il ne faut pas dépasser » (CFTC).

«Cela devient facile quand, le vendredi soir, vous avez fini la semaine, vous êtes prêt à partir, le client vient de téléphoner et lundi matin, il lui faut 53000 trucs devant la porte parce qu'il a ses trucs arrêtés et qu'il faut qu'il travaille et on nous dit: il va falloir travailler samedi, il va falloir travailler dimanche et si vous ne le faites pas, il ne va pas être livré, s'il n'est pas livré, il va nous enlever la commande et s'il nous enlève la commande, on n'aura pas de travail pendant 6 mois [...]. C'est du cinéma ça. Moi je dis qu'au chantage, il ne faut jamais céder. Si vous mettez le doigt une fois, c'est fini pour la vie, vous allez travailler tous les samedis, tous les dimanches et tout le temps, il y aura toujours ce truc à un moment donné. Vous pouvez avoir cette idée, vous dire, effectivement c'est vrai, puis après vous allez vous rendre compte que c'est quelque chose d'exceptionnelle et avec le temps qui passe, ça devient le normal, et on s'en sort plus » (CGT).

Un autre thème, celui du travail de nuit et du week-end, permet d'illustrer cette quête de la stabilité des repères temporels.

« Nous avons toujours été opposés et notre syndicat répond systématiquement non aux ouvertures des grandes surfaces les week-end. Nous n'aimons pas ces approches là. Il y a une approche travail-société qu'il faut quand même garantir, une symbiose entre les deux qui fait que si on privilégie trop le travail, c'est la cellule familiale qui se détruit, c'est aussi beaucoup de problèmes qui sont générateurs aussi de chômage. Si vous voulez, nous avons plus une approche plus humaniste des choses qu'économique, tout en sachant que dans certains endroits, peut-être c'est indispensable [...]. Le travail n'est pas un but en soi, il permet de vivre, de vivre correctement, mais si on fait du travail, si on s'amuse à travailler le samedi et le dimanche, si on donne des dérogations à ce genre de choses, c'est une conception de vie qui disparaît, c'est la cellule familiale qui à terme sera détruite, et je pense, à mon avis, que ce n'est pas la meilleure approche. Je pense qu'il y a d'autres formules de l'aménagement du temps de travail, sans en arriver là » (CFTC).

\section{II.3.C. Maîtriser l'usage du cadre temporel de travail et préserver son emploi}

La troisième caractéristique du référentiel de base concerne la maîtrise du cadre temporel des activités professionnelles. A l'insuffisance de temps pour soi et à la volonté de disposer de repères temporels stables et cohérents avec d'autres temps sociaux, les responsables syndicaux ajoutent la nécessité de maîtriser l'usage du cadre temporel du travail. La 
question ne porte pas sur le temps en soi que l'on pourrait traduire en termes de conflit entre l'aliénation par le travail et la jouissance par le temps libre, pour reprendre l'expression de Sue (1994), mais sur le caractère imposé des nouvelles règles temporelles. Non seulement ces nouvelles règles détruisent les repères temporels habituels, mais elles renforcent le contrôle de l'emploi du temps des salariés, de la disponibilité et de leur mobilisation. Au contrat de subordination du salarié à son employeur qui est inscrit dans le contrat de travail s'ajoute un contrat de soumission à la gestion de l'emploi du temps du salarié par l'employeur, notamment pour maintenir son emploi ou par crainte de le perdre. A propos de l'annualisation, le responsable CFDT observe que « les gens disent que, si ça continue comme ça, tout va partir à l'étranger et on ne va pas avoir notre boulot». De même le responsable CGC déclare : «Je pense que c'est assez intéressant et que ça évite des licenciements ou des contrats à durée déterminée ». A propos du temps partiel, les responsables syndicaux sont plus clairs : «Le travail à temps partiel, je pense qu'il faut que ça soit inscrit dans les accords d'entreprise mais que ce soit un temps partiel voulu et non imposé c'est le $1^{\circ}$ point. Le $2^{\circ}$ point est que ce ne soit pas un frein à la carrière, on doit avoir un suivi de la carrière de ces gens là. Si on l'a négocié, l'entreprise était d'accord pour le mettre en place donc il faut le suivre. Donc on est favorable au temps partiel.» (CGC).

Enfin pour le travail de nuit, il est accepté sous contrainte : "S'il n’y a pas de contrainte réelle et forte, je dis de façon naturelle que les gens n'ont pas envie de travailler la nuit [...]. Vous savez, tout se traduit en terme économique. Ce qui est bien la démonstration que si les gens gagnaient suffisamment pour s'éviter la prime de nuit et de week-end, ils ne feraient pas. L'attrait qui a été fait ce n'est pas, ce n'est jamais une volonté réelle, c'est un attrait économique [...]. Mais, si pour le même salaire il fallait choisir entre le jour et la nuit, faites-moi confiance qu'ils ne le feraient pas » (FO).

«Sur le travail posté, c'est pareil, j'en connais qui sont organisés avec leur époux. Ce n'est pas une vie familiale, un qui travaille la nuit, l'autre qui travaille le jour, on se laisse des petits mots sur la table, mais ça règle un tas de problèmes, tels la garde des enfants, l'économie des sous de crèche parce que ça répond à des difficultés, $33 \%$ de primes et puis le pouvoir d'achat [...]. Il y a celui qui cherche du travail, il arrive chez l'employeur, il a un contrat devant lui, le travail est posté. Si le type est au chômage, il ne va pas commencer à débattre avec l'employeur sur le travail posté. Dans sa tête ça fait six mois qu'il galère, il signera, c'est normal. Après, il y a celui qui a des difficultés : $33 \%$ de salaire en plus, ça l'arrange parce que ça va lui permettre de partir en vacances, un peu plus pour les gosses [...]. Je ne porte pas de jugement là-dessus. Je ne suis pas dans cette 
situation. Je suis dans la situation de l'activité revendicative sur des objectifs précis et je reste là-dessus »(CGT).

\section{CONCLUSION}

L'analyse des arrangements temporels, effectuée au chapitre précédent, nous a permis d'entrer dans la complexité de la négociation, mais aussi de faire ressortir que le temps de travail n'est plus traité pour lui-même, mais pour autre chose; il sert d'outil de gestion de l'emploi. Autrement dit, le temps de travail ne serait plus qu'un moyen de marchandage pour obtenir autre chose, notamment le maintien de l'emploi, comme si l'on glissait du temps de travail au temps de l'emploi. Il nous faut nuancer cette affirmation effectuée précédemment.

\section{Le temps de travail au centre du projet d'action de chaque organisation syndicale}

Dans ce chapitre, nous avons montré, premièrement, en explorant les référentiels négociatoires, que le temps de travail reste bien une préoccupation majeure des négociateurs, employeurs ou salariés : le temps de travail occupe dans les représentations véhiculées par les organisations syndicales une place centrale, d'autant plus intéressante, qu'elle nous donne à voir les contours de leur projet d'action. L'explicitation de ce qu'il y a de commun dans les représentations des organisations syndicales des salariés, nous a permis de montrer que le temps de travail doit être, (a) repensé dans le sens d'un allégement, car la pression temporelle est trop forte, (b) articulé avec les autres temporalités sociales, dont celle de la famille, pour limiter les conflits temporels, même si les raisons évoquées varient d'un syndicat à l'autre, (c) relié avec la question du maintien de l'emploi, même s'il faut se soumettre aux exigences de la flexibilité, enfin (d) un moyen pour développer plus de solidarité entre ceux qui ont un emploi et ceux qui en sont, plus ou moins durablement privés.

Autrement dit, on est loin d'une acceptation, passive ou active, de la logique de la production; mais la manière d'orienter l'action syndicale vers la logique de la vie quotidienne des salariés change : l'action syndicale s'oriente, de plus en plus, vers des logiques de compromis, dès que l'entreprise ou l'emploi sont menacés. L'incertitude du lendemain, l'insécurité sur les conditions matérielles d'existence amènent une plus grande variabilité des positions, un registre de réponses plus étendu, et souvent un résultat de la négociation incertain et fragile

Ce référentiel commun nous semble jouer un rôle dans la négociation : certes, cela ne signifie pas que ces «valeurs » se déclinent mécaniquement dans la négociation, mais 
qu'elles seront présentes et constituent en quelque sorte la toile de fond de la négociation. En référence à la notion de contexte organisé, élaborée par Friedberg (1993), nous interprétons la négociation sur le temps de travail comme un processus qui se déroule dans un contexte structuré par les représentations véhiculées par les acteurs; ce référentiel servirait le collectif à élaborer ses propres règles en fonction des contextes.

\section{Le déplacement des termes de l'échange : un profond changement des termes}

Deuxièmement, nous avons mis en évidence, la spécificité du référentiel de chaque organisation syndicale, pour déboucher enfin sur ce qui divise les organisations syndicales et sur ce qui les réunit. Afin d'éviter l'inventaire de ces convergences ou divergences, nous proposons de résumer les tensions qui expliquent la variété des positions : ces tensions sont en fait les pôles d'orientation de l'action syndicale, tels que nous les déduisons de l'analyse des référentiels négociatoires; ils constituent en même temps les enjeux de l'action syndicale.

La menace de licenciement fait accepter la flexibilité du temps de travail, c'est ce que nous avons vu. Jusqu'où ce chantage peut-il s'étendre ? Quel sera le seuil à ne pas dépasser pour que les organisations syndicales ou les salariés eux-mêmes continuent à coopérer et à négocier? Il y a bien là un déplacement de la frontière entre ce qui est acceptable et ce qui ne l'est pas, mais aussi une modification des termes de l'échange: il ne s'agit plus de répartir les gains de productivité, mais de contribuer à l'améliorer ; il ne s'agit plus de faire des accords locaux du type «donnant-donnant», mais de réviser les bases du projet d'action syndicale.

Les termes de l'échange se modifient et en même temps le seuil de l'acceptable ou de l'inacceptable se déplace :

- maintien de l'emploi (y compris sur des statuts différenciés) et temps de travail (flexibilité, rallongement de la durée du travail effective pour certaines périodes) ;

- disponibilité pour l'entreprise (professionnelle) et disponibilité personnelle (non professionnelle) ;

- protection de ses avantages acquis (notamment financiers, mais aussi de prise de congés) et partage (diminution de salaire, notamment par acceptation de la baisse des heures supplémentaires);

Ce changement de l'orientation de l'action syndicale ne signifie pas que tous les syndicats vont s'aligner sur la ligne de la production; cela signifie une modification du plan d'action qui est davantage insérée dans le contexte de la société où cohabitent fonctionnaires, chômeurs et salariés avec des statuts précaires. Il semble que ce contexte joue aussi pour 
réviser les positions antérieures : on voit mal dans le contexte de chômage et de précarité, les organisations syndicales défendre les heures supplémentaires ou les primes, (qui avaient été octroyées non pour rémunérer la pénibilité, mais pour limiter le nombre de personnes qui pourraient y être exposées). On retrouve ici les conclusions d'une enquête auprès de 3413 personnes effectuée en mars 1995, (Guillaume La Chaise, 1996). Si le travail reste une valeur fondamentale, c'est bien «la sécurité de l'emploi » qui est exprimée comme « le plus important dans la vie professionnelle». 


\section{CHAPITRE V \\ LE PARTAGE FLEXIBILISE DU TRAVAIL : \\ UNE ETUDE DE CAS EN ALLEMAGNE}




\section{PARTAGe DU TRAVAIL : DIVISER OU REPARTIR?}

La question du partage du travail peut recouvrir plusieurs significations toutes liées à la question de l'emploi. Certains l'associent à la réduction de la durée du travail : peu importe son contenu et les modalités de son application. En effet, on peut considérer que chaque réduction de la durée du travail dégage d'abord un certain nombre d'heures de travail, au moins potentiellement, pouvant servir à diminuer le chômage ou à stabiliser son niveau. D'autres associent le partage à la perte salariale, comme « sacrifice » des salariés pour maintenir l'emploi. Dans ces deux cas, c'est une conception d'un partage organisé collectivement qui, selon le Larousse (1988, p. 1341 ), « repartit un bien ou une masse de biens entre plusieurs personnes qui avaient toutes des droits sur l'ensemble de l'objet ou des objets partagés ». Cette interprétation définit le partage comme contractuel et comme un principe de justice morale (Zarifian, 1996).

Une troisième interprétation du partage s'intéresse plus à l'action de diviser. Ainsi, l'augmentation du travail à temps partiel constitue un partage imposé : «trop souvent présenté comme un outil de la conciliation entre vie familiale et activité professionnelle, il \{le temps partiel \}apparaît de plus en plus comme un instrument de la flexibilisation de l'emploi et de partage du chômage réservé aux femmes », (Maruani, 1996,p. 178) . Dans ce sens, le partage du travail est considéré comme : «l'action de diviser une chose en portions ou parties », (Larousse, 1988, p. 1341). Les conséquences de cette division du travail sont bien sociales puisqu'elles désignent les femmes dans le cas du temps partiel comme les perdantes du «partage ». Ces deux conceptions du partage, « diviser» et « repartir» le temps de travail sont aussi des pratiques d'arrangement temporel dans l'entreprise.

Nous réservons ici le terme de « partage » aux arrangements décidés collectivement sur la réduction de la durée du travail entre les salariés et ceux qui contrôlent le temps de travail : l'entreprise, la branche ou l'État. La réduction de la durée journalière, hebdomadaire ou annuelle du temps de travail dans le contexte du partage est un échange avec au centre la question de l'emploi. Le partage du travail incorpore pour nous l'idée de « sacrifices » afin de stabiliser l'emploi, dans le cadre d'un rapport de force peu favorable aux représentants des salariés. Le partage du travail salarié repose sur la négociation collective. Rien ne préjuge ici de la perte salariale réelle ou de la flexibilité concédée pour maintenir l'emploi. Il est tout à fait imaginable qu'une perte se transforme en gain ultérieurement. Il est 
possible aussi que la perte est durable et qu'elle participe à la dégradation des conditions de travail.

Avec l'accord de Volkswagen que nous avons étudié et que nous voudrions présenter, nous disposons d'un exemple qui dans le contexte les relations professionnelles allemandes a eu un caractère « d'événement». La question de départ a porté sur le licenciement prévu de 30.000 salariés : quelle solution trouver pour maintenir l'emploi de ces salariés?

L'arrangement trouvé va changer de manière « imprévue » les termes de la négociation du temps de travail. Ce qui a des conséquences sur la négociation de l'espace sociétal et interprofessionnel. Il ne s'agit donc pas d'une comparaison internationale FranceAllemagne terme à terme. Nous tentons au contraire de mettre l'accent sur la dynamique interne et externe d'un cas de partage du travail ou d'un événement qui va se diffuser dans un espace qui dépasse largement l'entreprise. La particularité de cette approche est donc d'analyser le partage du travail et la question d'emploi du point de vue de sa construction et diffusion. Il s'agit d'un seul aspect de la négociation du temps de travail en Allemagne, mais dont nous faisons l'hypothèse qu'il est central pour la compréhension des relations sociales à propos du temps de travail. Il montre toute la complexité d'un «modèle » des relations professionnelles en Allemagne et souvent à tort caractérisé comme mécanique, huilé, pacifié et sans exception. Nous nous intéressons à la question comment le partage se transforme et comment il est repris par d'autres niveaux de la négociation. Peut-il faire «boule de neige » face à un système des relations professionnelles allemandes plutôt « traditionaliste » en matière de négociation collective?

\section{LA SEMAINE DE 4 JOURS CHEZ VW : UN ACCORD EN DEUX TEMPS}

\section{II.1. Les relations professionnelles en Allemagne}

Deux caractéristiques principales nous semblent caractériser les relations professionnelles en Allemagne : (a) la négociation collective centrée sur la branche d'activité et (b) le poids important de l'organisation syndicale de la métallurgie (IG Metall) dans 1'histoire de la négociation du temps de travail.

Le niveau privilégié de négociation en Allemagne est la région et la branche. L'État apparaît ainsi en retrait, laissant aux syndicats et aux organisations patronales le soin de négocier les salaires et les conditions de travail (Tarifautonomie). L'accord d'entreprise, négocié entre les représentants des salariés élus au conseil d'entreprise (Betriebsrat) et la 
direction, constitue, quant à lui, plutôt un complément à une convention collective, concernant les modalités de sa réalisation. Les lois régissant les rapports de travail réservent aux conseils d'entreprises la possibilité de s'informer et d'influer sur les décisions prises au niveau de l'entreprise (Codétermination/Mitbestimung) ${ }^{31}$. Il en est de même au niveau de l'usine (ou établissement), lorsque l'entreprise comprend plusieurs sites autonomes de production, comme c'est le cas pour VW.

Le mouvement syndical apparaît à première vue moins divisé qu'en France, puisque unifié au sein d'une confédération unique: Le DGB (Deutscher Gewerkschaftsbund). En revanche, seules les organisations syndicales de branche ont le pouvoir de négocier. On note entre elles des orientations stratégiques divergentes. Dans le cas de la métallurgie, c'est le syndicat IG Metall qui tente de réaliser région par région les objectifs élaborés au niveau national.

Soulignons aussi que la position de Volkswagen est particulière dans le paysage des relations professionnelles allemandes, puisque l'entreprise n'appartient pas à l'union patronale régionale. Ainsi, la direction de l'entreprise peut négocier directement avec la direction régionale d'IG Metall des conventions qui lui sont propres (Haustarifvertrag) et qui seront appliquées dans les différents sites de Volkswagen suivant des modalités définies entre les conseils d'établissement et les directions de chaque site. C'est le cas de l'accord qui nous occupe ici et qui rompt avec les objectifs traditionnels poursuivis par IG Metall en matière de temps de travail.

\section{II.2. Chronique d'un accord éclair et fondateur : du 1er octobre au 15 décembre 1993}

Rappelons d'abord pour le cas de Volkswagen, l'importance du conseil d'entreprise général (Gesamtbetriebsrat) composé pour une large partie de militants IG Metall. ${ }^{32}$ Celuici constitue, dans un premier temps, l'interlocuteur privilégié de la direction de Volkswagen. En revanche, seule la direction régionale IG Metall peut entrer en négociation collective avec la direction de la Volkswagen AG. L'aperçu global du processus ayant conduit à l'adoption d'un accord sur une semaine de 4 jours chez VW se décompose en quatre étapes distinctes :

31 Sur ce point, voir les analyses sur la complexité et les contradictions de ce trait des relations professionnelles allemandes de: Hirsch-Kreinsen (1996).

32 Cette première étude de la négociation de l'accord VW a été entrepris avec Anne Labit (GRIS, Rouen), voir aussi Thoemmes \& Labit (1995). 
1) l'information sur la situation de l'entreprise par la direction générale et la demande du conseil général de l'entreprise à la direction d'élaborer un accord «maintien de l'emploi », 2) l'accord de principe sous la forme d'une convention collective, 3) la mise en œuvre de la semaine des quatre jours au niveau des sites selon des accords d'entreprises et 4) la réalisation et l'ajustement des différents modèles de la réduction de la durée du travail.

Pendant l'automne 1993, la direction VW a fait un pronostic sur l'évolution de l'emploi prévoyant une réduction des effectifs de 30000 salariés pour l'année 1994 et 1995 . Le démarrage des négociations entre IG Metall et la direction s'est fait sous l'emprise de cette prévision provoquant aussitôt la demande du conseil d'entreprise générale à la direction d'élaborer une alternative aux suppressions d'emplois. IG Metall décide alors d'entrer « officiellement » en négociation sur une convention collective « maintien de l'emploi » et fixe avec la direction une réunion pour le 11 novembre $1993^{33}$. Pendant celle-ci, la direction a argumenté sa position concernant l'évolution des effectifs dans l'entreprise avec des données structurelles et conjoncturelles. Elle a souligné les difficultés pour l'entreprise de «fonctionner » avec un niveau d'effectifs qui donne lieu à des coûts salariaux élevés face à la concurrence étrangère. Ensuite, la direction a présenté un texte « stratégique » qui rendait compte du souhait syndical, exprimé par le conseil d'entreprise général, de maintenir intégralement l'emploi en développant un modèle de la réduction du temps de travail de $20 \%$ dans l'entreprise, mais qui signifie des pertes proportionnelles sur les salaires à la même hauteur. Le modèle Hartz (1994) a développé trois axes opératoires pour la réalisation de cet objectif :

a) une réduction de la durée du travail à 28,8 heures, l'introduction simultanée de la semaine des 4 jours et une perte de salaire, b) l'entrée progressive des jeunes ouvriers formés dans l'entreprise et la sortie progressive des salariés proches de la retraite, c) des longues périodes réservées à la formation continue qui alterneraient avec des périodes de travail à plein temps.

La réaction d'IG Metall à ce papier «stratégique » s'est manifestée pendant cette première réunion autour de cinq principes que le syndicat voulait voir confirmés par une convention collective avec la direction: la convention collective doit concerner l'ensemble des salariés, même ceux qui sont employés hors convention comme les cadres de l'entreprise, la convention collective doit avoir une durée limitée sur deux ans jusqu'au 1/1/ 1996, des

33 Nous nous appuyons ici notamment sur un entretien avec la direction régionale de l'IG Metall (Hanovre, 8/94) et sur l'article de Schwitzer (1994). 
licenciements économiques doivent être interdits pendant cette période, le montant du salaire mensuel doit être maintenu pour les salariés, la perte sur le salaire annuel prévu de $20 \%$ doit être compensée partiellement par l'entreprise.

La diminution des salaires s'avère-t-elle être un tabou infranchissable? Les salariés sont-ils prêts à faire des sacrifices pour maintenir 30.000 emplois ? La rapidité de l'acceptation des quatre premiers points par la direction semble confirmer l'hypothèse d'un consensus préalable entre la direction et les représentants syndicaux sur une réduction des coûts salariaux « en douce ». Or, la compensation partielle de la réduction de la durée du travail n'a pas été retenue dans un premier temps. C'est sur cette question que s'engage le rapport de forces entre les acteurs

La nuit du compromis historique a eu lieu du 24 au 25 novembre 1993. L'accord obtenu montre qu'il y a effectivement compromis en fonction de deux exigences fondamentales : rationnement du travail et maintien de l'emploi. En fait cette relation met l'emploi au centre, mais se décline néanmoins selon trois processus concomitants :

- L'acceptation de la perte salariale qui caractérise le maintien de l'emploi en tant qu'objectif prioritaire (modèle 1 : pour tous),

- l'idée d'une progressivité de l'accès à l'emploi pour les jeunes encore en formation qui serait contrebalancé par la sortie progressive de l'emploi des salariés proches de la retraite, décrivant ainsi un processus de «solidarité inter-générationelle » qui aurait pour fonction de maintenir l'emploi des jeunes (modèle 2 : relais),

- la formation en tant que moyen de la réduction de la durée du travail et de développement permanent des qualifications des salariés (modèle 3 : bloc).

L'accord essaye de rendre compte de ces divers processus (accès à l'emploi, formation) qui construisent un ensemble de mesures ayant tendance à globaliser et à complexifier la question de l'emploi. Ces mesures qui restent pour partie non-réalisées, permettent néanmoins d'énoncer quelques questions, à propos de sa mise en œuvre dans les différents sites, sur les effets des pertes salariales annuelles et sur le nombre de licenciements évités, mais aussi sur la composition du cadre temporel des activités professionnelles qui résulte de ce partage du travail. Ce cadre possède une légitimité certaine : ni la direction, ni les syndicats ne voulaient revenir en arrière. Quels sont les facteurs de légitimité de la construction normative et quel est le seuil d'acceptabilité de part et d'autre? 
Si la réduction de la masse salariale satisfait globalement la direction, les syndicats ont dû demander la réalisation de quatre points qui permettaient d'accepter la réduction spectaculaire de salaire : l'interdiction de licenciements collectifs devient partie intégrante de la convention $(\S 5)^{34}$; la convention est provisoire et réversible, la norme des 35 heures reste en principe l'objectif, et la convention prévoyait d'y revenir fin 1995 (§7), la réduction du temps de travail s'applique à l'ensemble du personnel, les salaires mensuels restent stables par la suppression totale des primes annuelles, il y a compensation partielle de la perte financière pour les salariés.

\section{II.2.A. La « semaine de 4 jours » : les difficultés de la mise en oeuvre}

L'accord, négocié le 15 décembre 1993 au niveau central, a été suivi par une multitude d'accords entre les conseils et les directions de chaque site, concernant la mise en œuvre concrète de la «semaine de 4 jours ». Celle-ci pose en effet des problèmes de réalisation extrêmement différents suivant la nature et les «modes » de production. Mais, les accords passés sont aussi de courte durée et renouvelés dès qu'il y a modification de la situation, liée par exemple à la variation de la demande de tel ou tel produit. Enfin, notons que les aménagements les plus divers ont été imaginés afin de réduire le temps de travail de chaque salarié de 36 à 28,8 heures par semaine en moyenne, qui vont bien au-delà de la seule possibilité de répartir le travail sur 4 jours : 150 modèles peuvent être repartis autour de trois types d'aménagement (Thoemmes \& Labit, 1995) :

(1) temps de travail journalier «long» de 7,2 heures qui a comme effet de concentrer la réduction de la durée du travail sur le cinquième jour, la cinquième semaine ou sur un jour variable en semaine,

(2) temps de travail journalier « court » de 5,76 heures qui repartit la réduction de la durée du travail sur 5 jours,

(3) temps de travail journalier «moyen » de 6,17 heures où la réduction de la durée du travail est en partie reportée sur la troisième équipe et la troisième semaine du cycle.

On constate que l'effet de la nouvelle norme temporelle sur la répartition en période de travail et de non-travail n'est pas homogène, mais différentielle selon l'application de tel ou tel modèle. La diversité des situations et la complexité pour trouver le modèle «pertinent » a eu pour conséquence que la mise en œuvre de la solution VW s'est étalée dans le temps. Rapidement négociée dans les sites de Emden et de Salzgitter (Janvier 94), c'est là qu'elle trouve sa réalisation la plus « pure », puisque la semaine de travail s'y étale

34 Convention collective du 15 décembre 1993, §5: «Pendant la durée de la convention, des licenciements économiques sont exclus. [...]». 
sur 4 jours du lundi au jeudi pour l'ensemble du personnel. La « semaine de 4 jours » a ensuite été négociée puis mise en œuvre dans les sites de Kassel et de Braunschweig (Février 94), où elle prend des formes plus diverses ${ }^{35}$. C'est dans les usines d'assemblage de la Golf à Wolfsburg et du Transporter à Hanovre que les difficultés ont été les plus grandes.

A Wolfsburg, la «semaine de 4 jours » a été négociée le 16 mars et appliquée à partir du 4 avril, donnant lieu à des péripéties largement reprises par les journaux (entre autres de vastes embouteillages) dans une ville qui ne vit que par et pour Volkswagen. On trouve dans ce site environ 150 modèles différents de temps de travail permettant sa réduction de 36 à 28,8 heures $^{36}$.

\section{II.2.B. La «semaine de 4 jours » au département tôlerie de l'usine de Hanovre : la pénurie de main-d'oeuvre}

Nous aborderons maintenant plus en détail le cas de l'usine de Hanovre qui concentre bien des difficultés dans la mise en œuvre au quotidien de la réduction du temps de travail. La situation de Hanovre est «particulière » dans la mesure où le problème des sureffectifs ne s'est pas posé avec la même amplitude que dans les autres sites (réduction prévue des effectifs de $15 \%$ en deux années à Hanovre, contre une réduction prévue de $41 \%$ à $48 \%$ pour les sites de Wolfsburg et d'Emden, Hartz, 1994). La réduction du temps globalement travaillé de $20 \%$ «pèse » par conséquent plus lourd sur le site de Hanovre que sur d'autres sites. La question paradoxale qui se posera de plus en plus aux acteurs locaux sera : Comment maintenir le niveau de la production à Hanovre sans mettre en cause le principe de la réduction de la durée du travail ?

L'usine de Hanovre présente la particularité de fabriquer un produit, le « Transporter », dont la demande avait connu de fortes hausses. C'est dans ce contexte que doit se mettre en place la réduction hebdomadaire du temps de travail, qui ne doit en aucun cas donner lieu à une baisse de la production. Un modèle unique, auquel tenaient les représentants des salariés afin de ne pas casser les groupes de travail, a été négocié (accord d'entreprise) qui consiste en une semaine de 4 jours à 7,2 heures pour l'ensemble du personnel. Dans un premier temps la plupart des vendredis ont dû être travaillés par le

35 Chaque négociation s'est soldée par un accord d'entreprise et souvent par des notes internes qui règlent en détail l'application sur les sites. Une partie de ceux-ci est accessible en consultant Rosdücher, Seifert (1994). 36 Entretien : conseil d'établissement, Hanovre, 8/94. 
personnel de production directe afin que l'usine - qui a pu compter par ailleurs sur 180 salariés supplémentaires venus de Salzgitter pour satisfaire ses commandes ${ }^{37}$.

Les négociations entre le conseil d'établissement et la direction ont porté sur ce cinquième jour de travail (en général le vendredi). Chaque vendredi travaillé a pu être payé sous forme de salaire ou bien de repos compensateur. Par ailleurs, il n'a pas toujours été obligatoire. Dans ce cas, notons par exemple pour le département tôlerie, que seules 150 personnes sur 500 sont venues travailler sur cette base volontaire. «Beaucoup de salariés se sont très bien habitués à ce rythme de quatre jours », affirme un représentant du conseil d'établissement à Hanovre. «Le problème au département de la tôlerie est que nous n'arrivons pas à maintenir le niveau de la production. Nous avions besoin de 12 jours supplémentaires pour la production. On a fait un accord sur ces jours avec la direction ». Il précise l'enjeu pour l'usine de Hanovre : « Sinon 8000 clients ne peuvent pas être livrés ».

Dans ce contexte, certains représentants syndicaux à Hanovre (14 000 salariés) se plaignent « de ne pas peser aussi lourd sur ces décisions » que le site de Wolfsburg (36 400 salariés). ${ }^{38}$. Le conseil d'établissement avait insisté dans les négociations avec la direction locale à Hanovre sur la nécessité d'obtenir du personnel supplémentaire afin de pouvoir assurer la production dans le cadre d'une semaine de 4 jours. Il s'oppose surtout à la solution que propose de son coté la direction pour atteindre cette production, à savoir la mise en place d'un modèle de travail à trois équipes avec l'introduction d'une équipe de nuit, permettant un fonctionnement en continu de l'usine, mais qui risquerait de casser les groupes de travail constitués depuis des années. A plus long terme, la direction met en avant sa volonté d'améliorer la productivité de l'usine pour permettre de tenir un programme de production sans grever ses coûts en personnel. Comme le management de terrain en tôlerie le rappelle, cela ne peut se faire qu'avec du personnel qualifié, car améliorer la productivité revient essentiellement à accroître la disponibilité des nombreuses installations automatisées : la solution qui a consisté à faire venir du personnel de Salzgitter, ne lui est apparue à ce titre d'aucune efficacité, dans la mesure où les « savoir-faire » de cette usine de moteurs ne sont pas ceux qui sont nécessaires dans une tôlerie. Il insiste sur le problème de la mise en œuvre de l'accord à Hanovre : «La forte automatisation pose problème à tout le monde. Les cadences sont restées au même niveau, mais le temps de travail s'est réduit. Le niveau de la production est à peine tenable $»{ }^{39}$

L'encadrement de terrain observe une forte intensification du travail. L'accueil de personnel venant d'autres sites pour maintenir le niveau de la production à Hanovre est 
indispensable pour un fonctionnement à deux équipes. Or, les difficultés supplémentaires qui en résultent en termes de niveau de qualification et de capacité d'intégration dans un nouveau collectif de travail, ont amené les acteurs à revoir le choix d'un modèle unique sur 4 jours et organisé en deux équipes : «Dans la première phase de la semaine de 4 jour il y a eu de graves difficultés. Concernant la structure, l'introduction d'un tel système est toujours lié à des difficultés aussi, c'est surtout des fautes du management, ils ont été responsables pour ces graves difficultés, il y a eu des parties, des divisions entières qui ont été déplacées d'un jour à un autre, il y a eu des groupes de travail qui ont été divisés aussi, il y a eu des communautés qui font le trajet en voiture pour aller en entreprise qui ont été détruites », (un responsable de la direction régionale d'IG Metall, Hanovre).

La complexité de la question des effectifs avec le nouveau temps de travail peut faire qu'il soit demandé à certaines parties du personnel de changer de poste ou de site. Un changement de poste nécessiterait l'accord des acteurs locaux sur les procédures (convention collective, $\S 4.1^{40}$ ). Néanmoins, celui-ci constitue, selon nos interlocuteurs à Hanovre $^{41}$, un des problèmes les plus aigus de la mise en œuvre de l'accord qui peut contredire les attentes du salarié quand celui-ci n'est pas d'accord pour changer de poste, malgré l'accord du conseil d'établissement; de plus cette mobilité peut contredire les pratiques collectives de travail et les règles du groupe de travail du salarié. Bref, le changement de l'organisation temporelle a ici l'effet d'augmenter l'hétérogénéité et de déstabiliser les conditions de travail dans l'ensemble.

\section{II.2.C. L'accord et ses limites : ouverture d'un débat social sur l'irruption d'un nouveau paradigme de la négociation}

En revanche, malgré les contraintes que l'accord amène, la fragilité locale de l'accord proviendrait davantage d'un contexte où l'emploi serait de nouveau mis en question cause de l'inefficacité d'un partage du travail durable pour maintenir l'emploi dans l'entreprise. Il faut souligner que l'accord était solide dans la mesure où il a été négocié par des acteurs sociaux qui disposent de moyens forts pour normaliser et légitimer ses modalités diverses. Ils ont donné un cadre souple quant aux modalités d'application qui

\footnotetext{
40 Convention collective du 15 décembre 1993, § 4.1. : « Il peut être nécessaire dans l'intérêt du maintien de l'emploi de procéder à des changements de poste ou de site. Chaque salarié de l'entreprise est tenu d'accomplir la tâche attribuée. Les règles et la procédure sont élaborées par les directions et les conseils de l'établissement. Pour la question de l'attribution des tâches, il convient en particulier de prendre en compte les capacités, la qualification, la tâche précédente, le revenu et le lieu d'habitation du salarié concerné. [...]».

41 Entretien : agent de maîtrise, Hanovre, 8/94. Entretien : direction régionale IG-Metall, Hanovre, 8/94.
} 
délèguent une partie importante des décisions au niveau local ; d'où le sentiment de perte de contrôle du processus de la négociation éprouvé par d'autres directions régionales de l'IG Metall : la perte salariale et la flexibilité ne provoquent pas une adhésion spontanée à l'accord dans d'autres régions. Pour un représentant de la direction régionale d'IG-Metall à Berlin-Brandenburg : «Quand j’ai appris les résultats de la négociation chez Volkswagen, j'étais d'abord surpris, après choqué, mais j'ai fini par comprendre les collègues à Hanovre ». D'autres directions régionales de l'IG Metall réagissent en 1994 certes avec compréhension, mais craignent en même temps à moyen terme que ce mode de négociation ne soit imposé ailleurs. La difficulté de pouvoir contrôler ce processus au niveau régional voire national, la volonté de stabiliser l'ensemble des acquis sociaux et un scepticisme par rapport aux solutions rapides de «partage du travail » qui mènent à une augmentation de l'hétérogénéité des conditions de travail, leur fait préférer des réductions du temps de travail lentes, telles que celles demandées dans le cadre des négociations dans la métallurgie allemande de 1990 pour les 35 heures $^{42}$.

\section{II.3. Confirmation et seuil de décrochage du partage du travail}

L'accord de 1993 a eu une durée limitée fixée à 2 ans. Cette limitation a deux conséquences majeures. D'une part, cela signifie qu'une règle d'organisation est transitoire et temporaire. On pourrait même dire que l'instabilité est devenue une régularité qui emprisonne la règle dans le court terme. D'autre part, cette limitation signifie un climat d'incertitude sur la suite: les parties en présence ne vont-elles pas revenir sur leur position? Certes, un processus de négociation va se mettre en place pour tenter d'imaginer et de mettre en place des règles stables, mais aussi pour chasser l'incertitude qui accompagne le début d'une négociation. Donc le résultat est indéterminé. Nous voudrions, dans cette partie, aborder la poursuite de la négociation chez VW (après 1995), afin de voir si le partage du travail se maintient dans la négociation et sous quelle forme.

Deux éléments caractérisent cet accord : d'abord, il s'agit d'un accord spécifique et ensuite, il s'agit d'un accord limité dans le temps. Sous le premier aspect, la négociation a été isolée de l'ensemble des thèmes de la négociation, comme les négociations périodiques portant sur les salaires. Sous le second aspect, la négociation est « un contrat à durée déterminée », comme le dira un de nos interlocuteurs, la règle est donc partielle (l'emploi) et temporaire ( 2 ans). 


\section{II.3.A. Chronique de la négociation suivante : changement des termes de l'échange et conflit}

Le fait nouveau est que les parties en présence n'ont plus d'engagements à respecter : le syndicat se donne alors la possibilité d'utiliser tous les moyens de pressions dont la grève qui était interdite. La direction se donne la possibilité de demander quelques sacrifices supplémentaires aux salariés. La chronique de la négociation montre le «jeu » qui s'instaure entre ces deux acteurs.

(1) La première journée de négociations avec la direction a lieu le 9 août 1995. Pendant quatre heures, les parties négocient sur la proposition patronale d'introduire le samedi comme jour ouvrable. Cette proposition est refusée par la délégation syndicale.

(2) Les acteurs se retrouvent lors de la seconde journée des négociations qui a lieu le 15 août. La direction demande désormais un travail du samedi sans primes et, en plus, une durée maximale du travail de 48 heures comme limite supérieure d'une modulation annualisation.

(3) Lors de la troisième journée enfin, la direction propose une augmentation de la durée du travail d'environ 3 heures par semaine, par la suppression d'une partie des pauses payées, en échange du maintien de l'emploi dans l'entreprise. Cet échange devrait, selon elle, être limité sur deux ans. C'est à ce moment que la délégation IG Metall commence la mobilisation des salariés dans l'entreprise. Ainsi, après la fin de l'interdiction de grève fixée par le contrat salarial antérieur, IG Metall entraîne, selon ses propres sources, 22000 salariés dans des grèves d'avertissement à partir du 29 août 1995. Les équipes de nuit anticipent ce mouvement : ceci signifie en général un soutien solide de la base aux représentants des salariés. Une manifestation est organisée à Hanovre avec 25000 salariés.

(4) Lors de la quatrième session de négociation, le 29 et 30 août, qui durera 22 heures, la direction retire sous la pression des grèves sa demande du travail de samedi. Ensuite, le 4 septembre, sur l'ensemble des sites, 66000 salariés participent à une grève d'avertissement de 3 heures.

(5) La cinquième journée de négociations amène la délégation de la direction à proposer une augmentation des salaires de $3 \%$.

(6) Finalement, lors de la sixième journée, un compromis est trouvé : la direction concède l'interdiction de licenciements sur une durée indéterminée, mais elle obtient de pouvoir dénoncer l'accord au bout de 2 ans. Les salariés obtiennent une augmentation des salaires de $4 \%$, mais ils doivent désormais travailler 30 heures par semaine au lieu des 28,8 heures négociées précédemment. La modulation - annualisation est étendue et elle définit désormais une variation possible sur l'année entre 18,8 heures et 38,8 heures par semaine. 
Le travail du samedi ne devient pas un jour de travail habituel, mais les primes sont réduites de $50 \%$ à $30 \%$ (sources : IG Metall).

\section{II.3.B. Du conflit au compromis : les limites de la rationalisation sont dans la rue}

Ce que montre la négociation d'abord, c'est que la rationalisation du processus de travail est apparemment sans limites. La direction souhaite allonger la durée du travail, obtenir le travail du samedi, voire du dimanche, étendre l'annualisation. En même temps, elle crée les conditions de solidarité entre les salariés qui vont s'unir contre leur direction et se souder autour du syndicat dont ils renforcent la position. Ne peut-on pas alors considérer que les limites de la rationalisation sont dans la rue ? C'est du moins ce que les propos recueillis auprès des responsables syndicaux laisse penser. En effet, tant au niveau de la direction régionale d'IG Metall, qu'au niveau des responsables syndicaux en entreprise, notre recherche montre que la direction de VW veut avant tout rester exemplaire et qu'en aucun cas, elle voudrait qu'un conflit soit porté sur la place publique. On pourrait ajouter qu'IG Metall souhaite ne pas perdre la face et aboutir à un compromis. Aucun des deux protagonistes ne semblent avoir compté sur la mobilisation des salariés qui précipite 25000 salariés dans la rue, « devant la maison où on a négocié sur la prolongation de la convention collective », dira un responsable d'IG Metall à Hanovre.

La pression sur la délégation syndicale aboutit à un mouvement de solidarité des salariés visant à s'opposer aux demandes patronales sur la flexibilité du temps de travail jugées « illégitimes ». La flexibilité demandée concernant le travail du samedi fait porter le conflit sur la menace que constitue ce temps de travail au regard des autres temps sociaux :

Les grèves d'avertissement et les manifestations, en particulier, ont modifié les rapports de forces en faveur de la délégation syndicale. «C'est un jeu risqué, IG Metall appelle les salariés à une grève ou des grèves d'avertissements, ça veut dire aussi que les attentes augmentent de manière proportionnelle, c'est quelque chose que la direction sait aussi et ça fait baisser aussi la volonté de la délégation syndicale d'aller au compromis, c'est clair» (un responsable de la direction régionale d'IG Metall, Hanovre).

\section{II.3.C. L'emploi reste au cour de l'accord: interdiction de licenciements indéterminée et obligation d'embauche des apprentis}

Certes, le compromis adopté change de nature : désormais, il y a (a) un allongement de la durée du travail qui passe de 28,8 heures à 30 heures par semaine, (b) une modulation - 
annualisation qui permet à l'employeur de faire varier la durée légale du travail de 18,8 heures à 38,8 heures sans paiement d'heures supplémentaires et (c) le travail du samedi est moins rémunéré (de $+50 \%$ à $+30 \%$ par heure). Bref, la semaine de 4 jours éclate au profit d'un temps contrôlé par l'employeur. Mais, pour autant, peut-on conclure que la question de l'emploi disparaît? Qu'est-ce qu'IG Metall a gagné dans cette négociation? La réponse est claire : le syndicat a gagné (a) une interdiction indéterminée de licenciements, même si l'accord peut être dénoncé au bout de deux ans, (b) l'embauche de 1000 apprentis par an, enfin (c) une légère augmentation de salaire. Il nous semble donc, à première vue, que les termes de l'échange n'ont pas changé : les salariés consentent un effort supplémentaire du côté de la flexibilité en acceptant d'être disponibles pour l'entreprise dans le cadre de l'annualisation, en échange du maintien de l'emploi et d'une embauche de jeunes. C'est un accord dans lequel chaque partie a fait des concessions : il n'y a pas de « perdants », c'est ce que nos entretiens semblent relever.

Comme pour l'accord précédent, l'emploi reste par conséquent au cœur du résultat. Le partage du travail est maintenu. En revanche, la délégation syndicale part désormais de l'idée que des licenciements économiques sont exclus sur une durée indéterminée donc, de leur point de vue, les termes de l'échange ont changé puisque l'employeur s'engage à maintenir l'emploi de manière indéterminée ${ }^{4344}$ :

«VW n'a pas du tout été intéressée pour trouver un accord sur une durée indéterminée dans le temps. Alors, on a une interdiction de licenciements sur une période indéterminée. Mais, la direction voulait, au départ, seulement pour période d'une année ou de deux années. Un des problèmes le plus difficile à résoudre a été de décider pour combien de temps cette convention collective va être valable. Nous avons réglé, avec la convention collective, le maintien de l'emploi, non seulement pour les salariés qui veulent rester dans l'entreprise, mais aussi, et on n'en parle pas très souvent, pour les mille apprentis annuels qui sont dans l'entreprise et qui resteront après leur formation dans cette entreprise. Nous avons réussi à obtenir ça » (un responsable de la direction régionale d'IG Metall, Hanovre). ${ }^{45}$

43 « La convention collective modifiée sur la semaine de 28,8 heures est liée à l'exclusion de licenciements pour motif économique qui est prolongée à partir du 1er janvier 1996 avec une possibilité de résiliation au plus tôt le 31 décembre 1997. », Article 1.1.1., Verhandlungsergebnis zwischen des Verhandlungskommission der Volkswagen AG und der Industriegewerkschaft IG Metall-Bezirksleitung Hannover, 12 septembre 1995. Traduit par nos soins.

44 L'ancienne convention collective du 15 décembre 1993, § 5 : «Pendant la durée de la convention des licenciements économiques sont exclus. [...]».

45 «Les jeunes en formation (Auszubildende) se voient proposés de manière générale un contrat de travail après la réussite à leur examen final », Article 1.1, annexe 4, Verhandlungsergebnis zwischen des Verhandlungskommission der Volkswagen AG und der Industriegewerkschaft IG Metall-Bezirksleitung Hannover, 12 septembre 1995. Traduit par nos soins. 
Ce mouvement de solidarité autour de l'emploi, confirmé par la renégociation de l'accord, aboutit finalement selon les représentants syndicaux à une garantie indéterminée de l'emploi. Celle-ci est jugée compatible avec un investissement plus fort des salariés dans leur travail. Le maintien de l'emploi est, ici, vu par les syndicalistes comme une condition nécessaire de l'investissement des salariés dans leur travail

\section{II.3.D. De la semaine de 4 jours à 5 jours par semaine}

A notre surprise, lorsque nous revenons deux ans après sur les lieux de la négociation, nos interlocuteurs syndicaux ne parlent plus de la semaine de 4 jours. Si dans la première phase de l'accord en 1994, dans les deux plus grands sites, il existait effectivement un modèle de base de la semaine de 4 jours, aujourd'hui, nos interlocuteurs pensent que la semaine de 4 jours est finie. La distance que prennent les responsables syndicaux avec le mot d'ordre des « 4 jours », qui avait fait pourtant le succès médiatique de l'accord, semble désormais maximale. Un responsable régional déclare : «d'après ce que j’en sais, les 4 jours ne sont travaillés nulle part. La semaine des 4 jours a été, dès le départ, une mauvaise expression ; la semaine des 4 jours a été un mot d'ordre duquel nous nous sommes distanciés assez rapidement. Moi, je pense qu'il n'y a personne qui travaille 4 jours » (un responsable de la direction régionale d'IG Metall, Hanovre).

Les exigences productives et la situation sociale en Allemagne pèsent, en effet, sur le travail du week-end et nos interlocuteurs suggèrent que la semaine de 4 jours pourrait servir de Cheval de Troie pour le patronat afin de faire sauter, en particulier, le samedi comme jour de repos socialement reconnu.

« Non, ce n'est pas réaliste, on est dans une période où nous devons faire attention pour sauver le samedi et le dimanche ; la semaine de 4 jours n'est pas une bonne stratégie dans cette période. Une certaine partie du patronat a envie de combattre tout ce qui a l'air d'être un standard social, donc nous avons la pression du patronat sur ce travail de samedi, c'est très difficile pour nous de maintenir ça, donc ce n'est pas une discussion dans IG Metall aujourd'hui » (un responsable de la direction régionale d'IG Metall, Hanovre).

Le site de Hanovre a abandonné rapidement la semaine de 4 jours à laquelle tenaient pourtant les représentants syndicaux à l'époque :

« Je ne peux pas parler de pourcentage maintenant, mais ce que je peux dire, même s'il y a très peu de gens qui travaille 4 jours, c'est que quelques personnes dans la gestion du personnel travaillent 4 jours » (un responsable du CE de Hanovre, IG Metall). 


\section{II.3.E. Vers le travail du samedi ou vers un cadre pluriannuel ?}

La fin de la semaine de 4 jours aurait bien pu se terminer par une semaine temporaire de 6 jours, comme le démontre la volonté de la direction d'obtenir 12 samedis dans l'année sans prime supplémentaire.

Le travail du samedi qui a été à l'origine de la mobilisation des syndiqués, est finalement abandonné par les négociateurs, car il présentait un enjeu trop important pour la délégation IG Metall, dépassant largement le contexte de chez VW. La flexibilité ne s'impose pas d'elle-même, comme le montre le travail régulier de samedi qui est strictement refusé pour maintenir la concordance des temps sociaux chez VW, mais aussi afin de maintenir un rapport de forces sectoriel sur cette question. Enfin, la régularisation du travail de samedi aurait signifié la fin de la codétermination du conseil d'établissement du travail du samedi et la fin de sa surtarification.

«Le samedi est sacré pour IG Metall, et aussi pour les salariés. Le compromis qui a été obtenu était la pilule amère que nous devions avaler, c'était l'unification des tarifs pour des heures supplémentaires et pour le travail le samedi à $30 \%$, et la flexibilisation du temps de travail du lundi à vendredi », (un responsable de la direction régionale d'IG Metall, Hanovre).

Le compromis pour éviter le travail de samedi a alors porté sur l'acceptation d'une extension de la modulation - annualisation et sur une diminution des primes du travail exceptionnel le samedi. Ici encore, nous constatons que, non seulement la perte salariale est réajustée par le temps de travail, mais aussi que la réussite relative du blocage du travail le samedi se traduit néanmoins par une pression sur la flexibilité annuelle, le temps des marchés. Le compromis sur la modulation - annualisation ${ }^{46}$ est ressenti comme une concession que l'acteur syndical a faite à la direction.

Dans le site de Hanovre comme nous l'avions vu auparavant, on avait besoin de plus de temps de travail pour maintenir la production, sans recourir à des embauches supplémentaires. Le compte épargne temps ${ }^{47}$ est le dispositif utilisé pour réguler la modulation - annualisation :

\footnotetext{
46 « Dans les parties dépendant de la production, le temps de travail régulier peut être étendu à 8 heures par jour et à plus de 10 heures par rapport à la durée hebdomadaire régulière du lundi au vendredi », art. 1.1.1, Verhandlungsergebnis zwischen des Verhandlungskommission der Volkswagen AG und der Industriegewerkschaft IG Metall-Bezirksleitung Hannover, 12 septembre 1995. Traduit par nos soins.

47 « Afin d'assurer le respect de la durée annuelle du travail, un compte épargne temps individuel peut être introduit par accord d'entreprise, fonctionnant d'après le principe de l'épargne. », art. 1.1.1., Verhandlungsergebnis zwischen des Verhandlungskommission der Volkswagen AG und der Industriegewerkschaft IG Metall-Bezirksleitung Hannover, 12 septembre 1995. Traduit par nos soins.
} 
la convention collective renégociée stipule que « le paiement des heures supplémentaires ne peut intervenir que pour les heures qui dépassent les 35 heures $»^{48}$.

Le site de Hanovre semble déroger à la règle : seules les heures au-delà des 35 heures vont dans le compte épargne temps et les heures entre 28,8 heures et 35 heures sont rémunérées. Le commentaire de la direction régionale d'IG Metall qui dit ne pas être au courant par rapport à cette situation, est clair :

« Si vous faites plus de 28,8 heures pendant une période, vous devez avoir un équilibre sur l'année, c'est-à-dire qu'il faut que vous travailliez moins pendant une période pour arriver à cette moyenne-là ; s'il y a des heures supplémentaires, il faut prendre du repos » (un responsable de la direction régionale d'IG Metall, Hanovre).

En effet, la difficulté de la gestion des heures supplémentaires dans ce site montre que la structure temporelle du travail craque. L'intensité du travail augmente :

«Il faut voir que, si la réduction du temps de travail provoque de tels processus, c'est quand même dirigé contre l'emploi à terme, c'est ça le paradoxe. Nous avons des exigences très importantes au niveau de l'effort et de la densité du travail et je pense que, chez nous, il n'y a pas beaucoup de place pour intensifier encore l'effort du travail de la personne. On peut très bien déjà voir ce que fait une personne sur la chaîne, on a des informations là-dessus, donc on ne peut plus trop intensifier encore la charge du travail », (un responsable du CE de Hanovre, IG Metall).

Cette situation est vécue par les salariés du site comme un paradoxe devant la situation de l'emploi.

« D’un côté, on dit qu'il faut réduire le temps de travail pour sauver le plus d'emploi possible et, de l'autre coté, tous les jours on travaille plus. Là, les salariés nous disent: vous nous avez fait raccourcir les pauses, donc nous faisons plus maintenant sans que ça ait un effet sur l'emploi, mais plutôt le contraire. Il faut séparer, d'un côté, le temps de travail et, de l'autre côté, la politique sur l'intensité et l'effort », (un responsable du CE de Hanovre, IG Metall).

\section{II.3.F. Flexibilité et déstabilisation des collectifs de travail}

Il en ressort un souci constant des représentants syndicaux d'assurer une cohérence du cadre temporel. La stabilité du cadre temporel du travail, en particulier aux heures d'arrivée et de sortie, mais aussi la stabilité des collectifs de travail, ont été remis en cause par l'accord VW et ceci pose désormais le problème crucial à Hanovre.

48 Art. 1.1.3., Verhandlungsergebnis zwischen des Verhandlungskommission der Volkswagen AG und der Industriegewerkschaft IG Metall-Bezirksleitung Hannover, 12 septembre 1995. Traduit par nos soins. 
La recherche d'une stabilité maximale de ce cadre temporel devient alors un but ouvertement affiché dans une situation où le temps de travail éclate : « il faut avoir de la confiance, il faut pouvoir compter sur cet accord, donc il faut pouvoir planifier sur de longues périodes, il faut avoir des dates claires sur de longues périodes et, à l'intérieur de ces cadres-là, que ce soit quand même plus stable que jusque ici. Si on peut avoir de la flexibilité, il faudrait qu'on ait un maximum », (un responsable du CE de Hanovre, IG Metall).

En indiquant plusieurs dizaines d'accords d'entreprise conclus sur le temps de travail à Hanovre après l'introduction initiale de la semaine 4 jour, notre interlocuteur, pourtant spécialiste de la question du temps de travail, nous avoue que, parfois, il perd la notion du temps en vigueur dans l'entreprise. Le temps de travail est, en effet, soumis a une négociation permanente qui brouille les repères temporels :

«Moi qui ai participé à ces règlements, à ces négociations, etc.., je ne peux pas tout savoir, je ne peux pas me souvenir de tout de ce qu'on a fait, de ce qu'on a négocié. Les salariés ne sont pas toujours d'accord avec ça. Il nous disent: est-ce qu'on ne peut pas avoir un peu de stabilité ? Il y a toujours du mouvement, il y a toujours des choses, est-ce qu'on ne peut avoir quelque chose de simple dans ce monde un peu difficile, comme une heure d'arrivée régulière et une heure de sortie régulière? » (Un responsable du CE de Hanovre, IG Metall).

La perte de sens des arrangements temporels locaux, de plus en plus compliqués, montre, pour ce cas particulièrement difficile du site de Hanovre, les inconvénients du partage du travail :

L'approbation réelle de l'accord diminue. L'attitude aux élections professionnelles en témoigne ; un responsable syndical du site déclare :

«Si on prends en compte tous ces facteurs, selon les différents collèges pour ces élections, on peut dire que le degré réel d'approbation tourne autour de $40 \%$ à peu près. Ce sont les résultats les plus mauvais que nous avons eus »

\section{II.3.G. Travailler plus pour maintenir l'emploi}

Dans l'exemple analysé, il serait illusoire de croire que les gains seuls ont permis aux parties en présence d'aboutir à un accord. Il y a bien eu quelques pertes : les salariés ont consenti à travailler plus du fait du rallongement de la durée du travail. Mais, en travaillant plus, ils ont incontestablement contribué au maintien de l'emploi. On est bien en présence «d'un sacrifice», comme le dit un responsable syndical, pour maintenir l'emploi. Cette garantie de l'emploi est un «prix » à payer pour les salariés. La perte salariale doit être 
désormais considérée comme proportionnelle à la réduction de la durée du travail. On constate ici que l'ajustement entre le temps et le salaire passe par l'intensification du travail.

En effet, déjà le rallongement effectif du temps de travail à 30 heures est obtenu par la suppression d'une partie des pauses payées.

$\mathrm{Au}$ regard de cette dégradation du travail pour les salariés, comportant certes une nouvelle autonomie dans la gestion du temps, un responsable syndical du site de Hanovre pense qu'on ne peut pas aller plus loin dans le partage du travail et surtout des salaires :

"Nous, chez VW, nous avons l'impression que les collègues ne sont plus prêts, pour l'instant, à accepter des pertes salariales effectives supplémentaires par rapport à leurs revenus » (un responsable du CE de Hanovre, IG Metall).

Résumons donc brièvement les traits de l'arrangement VW «stabilisé ». La solution «Volkswagen » propose désormais une réduction de la durée du travail à 28,8 heures (30 heures de travail effectif) répartie sur 5 jours. Les salariés perdent, par le rallongement de la durée travail, environ $2 \%$ de leur salaire annuel brut comparé à la période suivant le premier accord. Le partage du travail, bien que maintenu, s'est transformé : la semaine de 4 jours est terminée et la flexibilité du temps de travail croît dans l'entreprise. La modulation - annualisation est devenue, avec le compte épargne temps, une manière flexible et individuelle de gérer le temps de travail. Dans les négociations, nous avons vu une forte pression de la direction pour imposer le travail de week-end. Les publications patronales par rapport à la renégociation de cet accord sont sans ambiguïté. Si, pour le premier accord en 1993, le livre de l'accord, publié par la direction de VW, s'intitule (Hartz, 1994) : « Chaque emploi a un visage, la solution Volkswagen », en 1995, le nouveau livre après l'arrangement stabilisé s'intitule (Hartz, 1996) : «L'entreprise qui respire : chaque emploi a un client - Maintien de l'emploi chez Volkswagen ». ${ }^{49}$

La fin de la semaine de 4 jours accrédite la thèse de la fin d'un temps de travail : d'abord, dans la partie où la réduction de la durée du travail pose problème. La modulation - annualisation est née de la nécessité de répondre aux besoins des sites sans sureffectifs. Mais par sa diffusion interne à l'entreprise, elle s'applique maintenant à l'ensemble des sites. En effet, l'usine qui « respire » est en réalité un concept de management, sous la forme d'une cascade de mesures de flexibilité temporelle basées sur la situation des commandes, comme le déclare le directeur du travail, représentant de la direction : 
«La base pour la semaine Volkswagen dans l'entreprise qui respire, est la situation des commandes. » $\left(\right.$ Hartz, 1996) ${ }^{50}$

Cette « respiration » de l'usine est assurée par un « poumon à 5 niveaux » :

- extension de la durée journalière du travail selon l'état des commandes,

- introduction d'équipes supplémentaire (de nuit par exemple),

- extension de la semaine de 4 jours à 5 jours,

- extension à la semaine de 6 jours avec samedi,

- mutation des salariés à d'autres postes ou sites.

Ces mesures, applicables dans l'ordre, définissent le recours à la flexibilité temporelle dans le cadre du partage du travail.

Pour autant, le partage du travail semble rester «légitime» dans l'entreprise et à l'extérieur. Plusieurs enquêtes effectuées semblent au moins en témoigner. Une enquête effectuée par FORSA sur 1001 personnes choisies de manière représentative en Allemagne montre que $51 \%$ des interrogés pensent qu'une réduction de la durée du travail avec perte salariale est capable de maintenir l'emploi ; $29 \%$ sont convaincus que ce n'est pas utile et $20 \%$ ne savent pas si c'est utile (cité dans Hartz, 1996, p. 102). Une enquête effectuée par l'Université d'Erlangen auprès du personnel de trois sites (Wolfsburg, Emden und Braunschweig) sur 2600 salariés montre, en juin 1995, un niveau de satisfaction comparable : 48,6\% se montrent «contents » ou «très contents » du premier accord obtenu, 34,7\% sont « contents et mécontents » selon l'aspect et 16,6\% sont « mécontents ou très mécontents » de l'accord (op. cité). Signalons toutefois que seul 6,2 \% disent que la perte salariale a été facile à gérer. Il semble, par ailleurs, que plus le revenu est élevé, plus il semble difficile d'accepter la perte salariale (op. cité). Ce qui peut faire réfléchir sur les conditions concrètes de la mise en œuvre d'une solidarité autour du partage du travail.

Que retenir de la renégociation du partage du travail chez VW ?

Premièrement, au regard du premier accord, les termes de l'échange entre employeurs et salariés changent. Dans le premier accord, le maintien de l'emploi s'échange principalement contre la perte salariale et la réduction de la durée du travail. Dans le second accord, le maintien de l'emploi s'échange contre la flexibilité du salarié enrôlé dans une augmentation de la disponibilité pour l'entreprise. Dans les deux cas, l'emploi sert de monnaie d'échange entre employeur et salariés. Deuxièmement, le temps de travail qui est négocié n'est plus le même. Dans le premier accord, il s'agissait bien d'une réduction de la durée du travail. Dans le second, il s'agit de la flexibilité temporelle. Tous les ingrédients de l'ordre temporel tourné vers la production sont présents : l'augmentation de la durée du 
travail (28,8 heures à 30 heures) et surtout la variation au cours de l'année, en fonction des impératifs de production; l'étalement des périodes de travail de 4 à 5 jours, voire de 5 à 6 jours et l'extension potentielle du travail en équipes successives. Ce type de résultat n'a rien de surprenant au regard des négociations menées dans d'autres pays comme la France. Ce qui est surprenant, c'est que le résultat fait à une première convention, prolonge, en les modifiant, les bases. Peut-on parler de la fin d'un temps de travail ou de la fin d'une illusion? La réponse n'est pas simple, car n'oublions pas l'essentiel : pendant la durée de la convention, les licenciements sont interdits.

\section{LA DIFFUSION DU PARTAGE « VW » EN AllEMAgNe : 1994-1996}

\section{III.1. Le cas de l'entreprise mécanique BERGO}

BERGO s'est trouvée confrontés au même problème que Volkswagen: fin 1993, la direction voulait licencier 700 personnes. Cette entreprise aux dimensions plus réduites a procédé à un arrangement temporel de type «VW ». Nous souhaitons, d'abord, montrer l'inspiration et les différences par rapport au modèle VW, ayant abouti à BERGO en 1994 à une solution temporelle permettant d'éviter les 700 licenciements. Cette étude a pour objectif de faire comprendre le point de vue externe à VW quant à la faisabilité d'importer la solution proposée par VW. Nous verrons que dans une situation totalement différente en particulier du point de vue des relations professionnelles, on aboutit à une solution différente, mais comportant les mêmes termes d'échanges et de conflits.

L'accord conclu chez BERGO peut être décrit rapidement : La baisse de la durée du travail de 35 heures à 32 heures évite 700 licenciements annoncés. En revanche, les salariés perdent $5 \%$ de leur revenu mensuel et doivent être plus disponibles : une modulation annualisation est introduite permettant de faire varier la durée du travail entre 24 heures et 48 heures hebdomadaires. Nous trouvons donc, en principe, tous les ingrédients du partage du travail de l'accord VW.

\section{III.1.A. Contexte et chronique de la négociation}

Notons, tout d'abord, la particularité des relations professionnelles chez BERGO : le CE est partagé entre des représentants syndiqués et ceux qui ne sont plus syndiqués à IG Metall ; c'est, en effet, l'attitude d'IG Metall par rapport aux menaces de licenciements qui a motivé des représentants à quitter IG Metall. L'histoire de la négociation chez BERGO commence avec la demande de la direction (fin 1993) de pouvoir licencier 700 salariés sur 1700 pour faire face au sureffectif de l'entreprise. Le conseil d'entreprise, l'institution représentative des salariés, refuse cette demande de la direction et propose de trouver une 
solution passant par la réduction de la durée du travail. C'est ainsi que commence, autour du maintien de l'emploi, la phase « chaude» de la négociation du temps de travail qui durera 6 mois. Les relations professionnelles sont donc très tendues pendant cette phase de la négociation. La production sur le site s'en retrouve affectée :

«C'était le temps où le directeur de l'entreprise a pris sa voiture, est rentré dans l'entreprise et est allé avec sa voiture jusqu'au bureau. Il a ouvert la porte de sa voiture et il est allé tout de suite dans son bureau. Il faut dire aussi que, pendant la période où on discutait du licenciement de 700 personnes, il y a eu des grèves qui n'ont pas été déclarées, qui n'ont pas été officielles, il y a eu des arrêts de travail », (un responsable du CE nonsyndiqué).

\section{III.1.B. Mythe et réalité de l'accord fondateur chez VW pour les acteurs de BERGO}

En effet, cette situation d'une demande syndicale d'un partage local et flexible du temps de travail qui ressemble à celle observée chez VW, s'est inspirée de cette solution, mais en diffère dans des points centraux: (1) un salaire mensuel moins élevé, (2) l'absence de primes annuelles de l'importance de chez VW et dont la suppression avait rendu possible la stabilisation des revenus mensuels, (3) un contexte de relations professionnelles défavorable au partage local du travail, du fait de la centralisation des négociations collectives sur la région. Ces trois points font de l'accord VW un schéma difficilement reproductible à l'identique, malgré la demande explicite des salariés de recourir à une solution de «type VW ».

Deux différences majeures s'opposent donc à l'application de la solution VW. D'une part, la faiblesse des primes annuelles empêchent de pouvoir stabiliser les salaires mensuels pour gérer la perte salariale. D'autre part, le système des relations professionnelles dans l'entreprise n'est pas le même. La direction de BERGO appartient contrairement à VW à l'organisation patronale régionale. Les acteurs sont donc obligés de passer par la négociation au niveau régional pour se mettre d'accord sur un cadre sous la forme d'une convention collective «maintien de l'emploi ». Rappelons cette particularité que VW pouvait négocier ces conventions collectives indépendamment.

\section{III.1.C. La solution retenue : partage du travail, perte salariale et flexibilité}

Ces deux difficultés vont accompagner la négociation du temps de travail jusqu'au compromis en août 1994. Nous proposons d'abord de regarder le résultat de l'accord, notamment pour voir comment le problème de la perte salariale et du contenu du partage du travail ont été résolus, avant de revenir à la question centrale de la régulation du temps 
de travail et de la difficulté de construire ce partage local du travail dans le cadre du système des relations professionnelles en Allemagne.

La direction qui avait décidé de licencier chez BERGO obtient du conseil d'entreprise l'offre d'un partage flexible du travail et accepte sous la pression des actions en entreprise un accord sans licenciements. L'organisation syndicale propose donc bien de la flexibilité pour la main d'œuvre en échange du maintien de l'emploi, un représentant du CE (nonsyndiqué) déclare :

C'est ainsi que le compromis d'août 1994 établit le cadre des 32 heures. L'accord et ses avenants sur les deux années suivantes stipulent que « la durée individuelle et régulière du temps de travail est réduite à 32 heures dans les parties production et qualité. Cette règle vaut du 1er février 1996 au 31 décembre $1999 »^{51}$.

\section{III.1.D. Les 32 heures, un cadre flexible pour les durées, mais rigide pour les salaires}

Une gestion des heures supplémentaires à la fois souple, mais contrôlée par un compte épargne temps, permet dans certaines limites de déroger à la durée de 32 heures.

Un responsable du CE précise :

Dans la pratique, cette solution a été largement utilisée provoquant quelques satisfactions des salariés dans cette gestion plus autonome du temps de travail : 1 a seconde raison pour laquelle les 32 heures restent une durée théorique concerne l'introduction d'une modulation - annualisation proprement dite, autour des limites légales du temps de travail : 10 heures par jour et 48 heures par semaine, et définissant ainsi un couloir entre 24 heures et 48 heures hebdomadaires, combinées avec le compte épargne-temps :

Notons qu'aucune loi n'est nécessaire en Allemagne pour procéder à la modulation annualisation ou encore pour introduire un compte-épargne temps. C'est, en effet, en dehors des règles définies dans les conventions collectives, le conseil d'entreprise qui codétermine la répartition du temps de travail. ${ }^{52}$ Cette véritable annualisation voir pluriannualisation du temps de travail réellement concédée par la conseil d'entreprise est associée au partage du travail. Contrairement à VW, le salaire mensuel est réduit, mais l'entreprise compense partiellement la perte salariale. Employeurs et salariés se partagent la perte salariale et l'emploi est maintenu sur le site.

Le processus de solidarité est ici, comme chez VW, une contrainte collective dont l'adhésion n'est pas spontanée. Le travail des syndicats sur la légitimité des normes (de l'emploi) fait passer le «sacrifice » sur les salaires. Cette attitude de compromis avec la

51 Protokollnotiz zur Betriebsvereinbarung über Arbeitszeit, art. 2.1., traduit par nos soins.

52 Le paragraphe 87, article 2 du Betriebsverfassungsgesetz de 1988, p. 413, stipule que : «Début et fin du temps de travail journalier, pauses incluses et répartition du temps de travail sur les journées de la semaine sont co-déterminés par le conseil d'entreprise », traduit par nos soins. 
direction ne peut pas être considérée comme «automatique ». L'enjeu de l'emploi surplombe la discussion sur le temps et le salaire.

La légitimité de l'accord n'est donc pas la plus grande autonomie des salariés dans la gestion de leur temps, ni la réduction elle-même. L'emploi reste ici le facteur déterminant. Le conflit sur l'emploi pourrait resurgir une fois cette période de l'accord révolue, comme nous l'avons vu chez VW. La légitimité de l'accord est étroitement liée à la garantie de l'emploi. Les avantages obtenus par la direction dans le domaine du temps de travail, mais aussi par rapport à la masse salariale, pourraient se retourner contre elle en cas de mise en cause de l'emploi et ce d'autant plus que les sacrifices effectuées par les salariés sont jugés importants.

\section{III.2. L'importance des régulations intermédiaires pour la diffusion du partage du travail}

\section{III.2.A. Bergo : un partage du travail dans le dos d'IG Metall}

Le problème principal de la conclusion de l'accord est l'absence de convention collective de référence au moment de l'application «maintien d'emploi» qui rend impossible l'accord d'entreprise. Entre le jour de la signature de l'accord et son application en 1996, en opposition au $\mathrm{CE}$, la direction régionale d'IG Metall n'avait pas envisagé de renégocier la convention collective régionale sur le maintien de l'emploi permettant de baisser la durée du travail en dessous des 35 heures chez BERGO. Cette convention collective avait été conclue peu après l'accord VW en Bavière, afin de permettre des partages locaux du travail. La convention collective devait venir à son échéance en novembre 1995, donc trop tôt pour le compromis de chez BERGO. La question est alors : comment est-on passé à la prolongation de cette convention collective qui devait constituer la base du partage local du travail?

A la demande du CE, IG Metall refuse la négociation de la convention collective. La direction de Bergo aussi ne semble plus favorable au partage du travail. IG Metall et la direction de BERGO qui constitue un membre puissant dans l'organisation patronale régionale, hésitent et veulent gagner du temps. Le conseil d'entreprise de BERGO adopte alors une stratégie afin de forcer l'organisation patronale, mais aussi IG Metall, à prolonger la convention collective pour le site afin d'appliquer le partage du travail. Le directeur nonsyndiqué de la représentation salariale en entreprise raconte comment il a ramené les négociateurs patronaux et syndicaux à la table de négociation :

« L'organisation patronale avait dit que c'était IG Metall qui bloquait et IG Metall m'avait dit que c'était l'organisation patronale qui ne voulait pas bouger. J'ai dit que, si nous ne 
pouvions pas arriver à signer ce papier, j'irai sur la scène publique et je dirai clairement qu'il y avait des personnes ici qui prétendent d'agir au nom des salariés, alors qu'elles font exactement le contraire. Donc, j'ai d'abord parlé avec un journaliste d'un journal local, je lui ai expliqué comment ça se passait et il a publié un petit article, c'était convenu comme ça et c'est paru dans le journal. En fait, parallèlement, je suis allé voir la direction de BERGO et j'ai parlé avec eux. Je leur ai dit, si vous souhaitez que votre organisation patronale signe ce papier, donc si vous voulez conclure l'accord sur le temps de travail avec nous, vous devriez amener votre organisation patronale à signer ce papier. J'ai pu avoir accès à des informations comme quoi l'entreprise BERGO a eu des commandes récentes assez importantes pour un produit et que ça coïncidait avec l'annonce des 700 personnes licenciées. On pouvait dire que la direction n'avait pas dit la vérité, parce qu'ils ont toujours dit: on n'a plus de commandes, ça va mal. C'est très important d'être bien renseigné pour être pris au sérieux par la direction ».

Cette pression sur les acteurs régionaux a finalement payé et la convention collective est prolongée au niveau régional jusqu'en juillet 1996 et pour l'entreprise BERGO jusqu'au 31 décembre 1999. Il s'agit ici d'une procédure tout à fait inhabituelle dans le contexte des relations professionnelles allemandes. Contre toute attente, la convention collective « maintien de l'emploi » dans l'industrie métallurgique régionale stipule dès lors que : « la possibilité de l'accord «maintien de l'emploi» entre l'organisation patronale et la direction régionale IG Metall est prolongé du 16 novembre 1995 au 31 décembre 1999 pour le seul (!) site de BERGO ». ${ }^{53}$

Cette négociation est donc très complexe au vue de la multiplicité des acteurs, conseil d'entreprise syndiqué et non-syndiqué, organisation patronale et syndicale de la région, direction centrale et locale de BERGO.

Le fond du problème semble être lié ici aux formes de régulations, et notamment à la difficulté de décentraliser les relations professionnelles allemandes pour conclure des accords locaux touchant au partage du travail. Néanmoins, cette question de la régulation sociale se confond ici avec la question du temps de travail. En effet, deux positions théoriques s'affrontent à l'intérieur des représentants des salariés : ceux qui sont prêts à faire des concessions de plus en plus importantes en vue de flexibiliser le temps de travail pour maintenir ou développer de l'emploi et ceux qui préfèrent garantir la stabilité du cadre temporel, s'il le faut, au détriment de l'emploi.

53 Tarifvertrag zur Beschäftigung der bayrischen Metallindustrie, 16 novembre 1995, art. 2 et Anhang, Seite 4, traduit par nos soins. 


\section{III.2.B. La montée des régulations intermédiaires du temps de travail en Allemagne depuis VW}

L'accord «Volkswagen » a contribué à modifier les termes dans lesquels on discute de la réduction de la durée du travail et du maintien de l'emploi en Allemagne. L'expression la plus immédiate de cette influence est la possibilité de négocier un partage du travail « local», dans la convention collective de la métallurgie et de l'industrie électronique de 1994. Si le contexte local amène les acteurs à vouloir négocier des réductions supplémentaires de la durée du travail pour maintenir l'emploi dans l'entreprise, cela se fera sur une base de moins de 35 heures avec réduction partielle ou proportionnelle du salaire. Un responsable syndical de la direction régionale de IG Metall à Hanovre déclare : « Aujourd'hui, on peut dire que la situation a changé et, bien sûr, tout le monde ne voit pas ça d'une manière positive, mais tout le monde est obligé de parler de ce thème».

Pour la première fois en 1994, une convention collective régionale «maintien de l'emploi » permet d'abaisser localement la durée du travail jusqu'à 30 heures par semaine, avec perte salariale en échange d'une garantie d'emplois. Cette solution s'était inspirée du modèle VW. Lors d'un entretien avec un responsable de la politique tarifaire du patronat allemand $^{54}$, il nous confirme un recours important des entreprises de la métallurgie à ces partages locaux du travail : «10\% des sites de la métallurgie font actuellement usage de ces conventions collectives et ce sont à peu près aussi $10 \%$ des salariés de la métallurgie qui sont concernés $»$.

Si ces chiffres sont exacts, sur les 3,5 millions de salariés du seul secteur de la métallurgie, déjà 350000 salariés seraient concernés par cette forme de partage du travail, alors que d'autres secteurs ont repris cette initiative à leur compte.

En revanche, un bilan de la diffusion de l'accord dans le secteur automobile s'avère plutôt décevant. La diffusion réelle du modèle VW a eu peu de succès dans l'industrie automobile. Le fait que les autres constructeurs automobiles ne suivent pas la solution de VW montre que la diffusion du partage n'est pas automatiquement lié au secteur d'activité. En tous les cas, la politique syndicale d'IG Metall consiste à ne pas généraliser une règle dont ils n'ont pas l'assurance de sa totale pertinence.

\section{III.3. Le niveau global s'approprie l'arrangement VW pour le généraliser}

L'un des grands objectifs d'IG Metall à partir des années 80 a été la réduction du temps de travail à 35 heures hebdomadaires sans perte de salaire. Les conditions de départ de cette

54 Entretien mené en avril 1996 avec un responsable de la politique tarifaire au Bund Deutscher Arbeitgeber (BDA), Cologne, traduit par nos soins. 
négociation semblent difficiles dans la mesure où même les 40 heures n'ont eu le caractère de norme légitime et respectée (c'est-à-dire appliquée par plus de $90 \%$ des salariés) qu'en 1978 (Bosch \& Lallement, 1991). Il aura fallu trois gros conflits dans la métallurgie, accompagnés de grèves dures, en 1984, 1987 et 1990, pour fixer progressivement la durée du travail à 35 heures par semaine sans perte de salaire. La dernière étape de ce processus, le passage de 36 à 35 heures, entre en vigueur à partir du $1^{\mathrm{er}}$ octobre 1995.

Ce long combat d'IG Metall illustre bien le fait que la réduction du temps de travail en Allemagne est le résultat de conflits et de négociations entre syndicats et organisations patronales et non de l'établissement de règles juridiques par L'État (IG Metall jouant ici un rôle moteur pour l'ensemble du mouvement syndical).

Mais la réduction négociée de la durée du travail dans la métallurgie comporte un second volet : celui de la flexibilisation des durées du travail. Dès 1984, l'organisation patronale (Gesamtmetall) a obtenu la flexibilisation des durées collectives et individuelles du travail (en respectant la durée moyenne de 38,5 heures sur 2 mois, l'entreprise peut moduler la durée d'une semaine de travail entre 37 et 40 heures). Cette première ouverture des syndicats va inaugurer une dynamique conventionnelle autour de la question de la flexibilité temporelle, dont les résultats rappellent ceux des différentes lois sur la modulation du temps de travail en France ${ }^{55}$. Ainsi, les conventions collectives successives de la métallurgie étendront progressivement la durée de la période de variation possible de l'horaire individuel de travail : 6 mois en 1987, puis 12 en 1994. La durée moyenne hebdomadaire diminue (37,5 heures en 1987, 36 heures en 1994). Les marchandages entre IG Metall et les organisations patronales (réduction contre flexibilité des durées du travail) se manifestent en 1990, lors des négociations sur la convention collective cadre de la métallurgie. IG Metall obtient de ces négociations la réduction de la durée du travail de 36 à 35 heures pour 1995, mais elle doit payer « le prix » sous la forme d'une différenciation des durées du travail dans l'entreprise. Depuis 1990, entre $13 \%$ et $18 \%$ des salariés d'une entreprise, selon la région considérée, peuvent travailler jusqu'à 40 heures par semaine. La réalisation de cette demande patronale qui peut allonger la durée du travail pour une partie des salariés sous forme de contrats individuels, constitue un des éléments-clefs de la flexibilité des durées du travail à l'heure actuelle (Promberger, 1994). Comme nous avons vu d'autres formes de flexibilité apparaissent depuis dans les entreprises : l'annualisation et la pluri-annualisation. 
En 1997, IG Metall discute sur sa stratégie au niveau national pour les négociations de fin 1998. Ayant obtenu la semaine des 35 heures en 1990, de nouvelles réductions sont proposées en 1997. Contrairement aux négociations des années 1980, IG Metall voudrait maintenant abaisser la durée conventionnelle du travail de 35 à 32 heures en acceptant, s'il le faut, une perte salariale. La baisse du chômage passe par le « sacrifice » sur le salaire. Même si IG Metall rencontre l'hostilité des organisations patronales, mais aussi d'une partie des membres d'IG Metall, c'est dans ce sens du moins que son directeur Zwickel voit l'évolution en Allemagne : «Sans une réduction importante de la durée du travail, la division du chômage par deux est impossible à atteindre pour l'année $2000 »^{56}$. Le vicedirecteur constate néanmoins que le processus de la flexiblisation est incontournable : « la situation de 1985, quand nous avions forcé le passage de la réduction de la durée du travail, n'est pas comparable avec la situation d'aujourd'hui. [...]. Cela vaut, avant tout, pour la réalité de l'entreprise caractérisée par la différenciation et la flexibilité du temps de travail. Une inversion de ce processus de différenciation n'est pas réalisable avec la semaine des 32 heures $»^{57}$. La direction d'IG Metall pense néanmoins que ce partage différencié ou flexible du temps de travail à 32 heures peut permettre de continuer la réduction de la durée du travail au profit de la création d'emplois. Comment peut-on expliquer ce virement de stratégie chez IG Metall ? N'avait-il pas, il y a dix ans, mis tout les moyens pour éviter la perte salariale en cas de réduction de la durée du travail ? Qu'est ce qui prouve que les 32 heures créeront des emplois ? En réalité, la positon d'IG Metall n'est pas homogène et sa direction a voulu polariser le débat 18 mois avant la prochaine négociation avec le patronat (1999).

Le directeur d'IG Metall justifie ces propositions et le changement de la position : "Nous disons que ce sont des propositions. Parlons-en. Si les employeurs nous disent que les 32 heures seraient un coup mortel pour l'Allemagne, VW prouve le contraire. Ils ont une durée conventionnelle du travail de 28,8 heures dans la semaine et sont dans une meilleure situation que jamais ${ }^{58}$. La durée du travail chez VW est citée comme un argument prouvant la faisabilité d'une réduction de la durée du travail à 32 heures au niveau national : «Volkswagen est le meilleur exemple que la réduction de la durée du travail ne constitue jamais la faillite d'une entreprise ou un danger pour l'économie ». ${ }^{59}$

56 Handeslblatt, Duesseldorf, 25 juillet 1997,traduit par nos soins. 57 Handeslblatt, Duesseldorf, 20 mai 1997, traduit par nos soins. 58 IG Metall, Das Monatsmagazin, no 5, 1997, p. 4, traduit par nos soins 59 Handeslblatt, Duesseldorf, 30 avril 1997, traduit par nos soins 


\section{Partage et fin d’un temps de travail en Allemagne?}

\section{IV.1. VW introduit un paradigme concurrentiel de la négociation du temps de travail qui se diffuse en Allemagne}

Le débat sur la flexibilité du temps de travail continue d'agiter considérablement les acteurs sociaux en Allemagne. Les organisations patronales, se référant à la concurrence européenne, demandent toujours plus de flexibilité, tandis que les syndicats se montrent de plus en plus prêts à échanger cette flexibilité contre la garantie du maintien de l'emploi. La disparition d'anciennes normes temporelles et leur remplacement par de nouvelles se font dans un mode de négociation différent.

Entre 1984 et 1990, la réduction du temps de travail s'échange contre :

des augmentations salariales modérées (par rapport à la phase précédente) ;

la flexibilisation des durées du travail.

La phase actuelle, elle, est caractérisée par le couple « réduction du temps de travail et maintien de l'emploi » qui s'échange contre :

la diminution proportionnelle du salaire ou une faible compensation de cette perte ;

la flexibilisation des durées du travail.

On aurait donc à faire en ce milieu des années 90 à une modification des termes de l'échange par rapport aux années 80. Le maintien de l'emploi constitue l'enjeu central de la négociation et la réduction de la durée du travail semble être le moyen pour y parvenir. Cinq caractéristiques nous indiquent l'existence d'un autre paradigme de la négociation du temps de travail en Allemagne : (1) l'acceptation de la perte salariale, (2) l'augmentation de la flexibilité, (3) le centrage sur l'emploi, (4) la percée des régulations intermédiaires dans les relations professionnelles allemandes, (5) la mise en cause et le conflit autour des temporalités sociales.

\section{IV.2. L'acceptation de la perte salariale}

Une première caractéristique de l'accord fondateur de VW concerne l'acceptation d'une perte salariale importante : environ $18 \%$ du salaire annuel. Les effets cumulés des deux accords chez VW aboutissent à une perte globale de $20 \%$, ce qui signifie que le partage du travail (moins $20 \%$ pour tous) est entièrement financé par les salariés. Le 
partage est l'affaire des salariés qui en subissent les contraintes financières. Ce modèle VW, nous l'avons vu, ne peut s'appliquer à l'identique dans des contextes différents. Ainsi, chez BERGO, l'introduction du modèle VW est demandée par les salariés dans une situation de menace sur l'emploi, mais elle s'avère difficile au regard des salaires moins importants et surtout au regard de l'impossibilité de garantir le niveau des revenus mensuels. La baisse salariale est moins importante (moins $5 \%$ ), mais elle s'applique sur le mois. Cette perte salariale, dans les deux cas, ne fait sens que par rapport à l'emploi maintenu et peut donc être interprétée comme un objet d'échange : il s'agit d'un sacrifice que les salariés sont prêts à échanger contre l'emploi pour tous. La perte salariale joue subjectivement (du point de vue des représentants syndicaux) un rôle tout à fait secondaire : du temps ce n'est pas de l'argent.

\section{IV.3. La flexibilité augmente}

L'accord fondateur chez VW avait demandé aux salariés un second sacrifice : l'annualisation de la durée du travail. L'accord suivant chez VW avait confirmé cette tendance en étendant la variation possible de la durée hebdomadaire du travail de 18,8 à 38,8 heures par semaine. La disponibilité des salariés s'accroît et dans le site que nous avons analysé, nous avons montré l'illusion de la semaine de 4 jours : il n'était pas possible de s'appuyer sur ce modèle, car les temps de production avaient demandé la répartition du travail sur 5, voire sur 6 jours. En effet, dans aucun site des 6 sites de VW, la semaine des 4 jours a survécu. Chez BERGO, nous avons vu que le partage du travail donne lieu à une flexibilité de niveau comparable. L'annualisation est introduite dans l'entreprise et la durée du travail peut désormais varier entre 24 et 48 heures par semaine. $\mathrm{Du}$ point de vue de chez VW, nous avons vu que la perte salariale s'exporte plus difficilement que la flexibilité.

\section{IV.4. Le paradigme du partage met au centre l'emploi}

La menace sur l'emploi motive bien entendu tous les accords que nous avons analysés dans ce contexte (VW1, VW2, BERGO). L'accord fondateur de chez VW nous surprend d'abord par la dimension du problème : 30000 salariés (sur 110000 ) à licencier. L'accord fondateur va essentiellement financer le maintien de l'emploi par la perte salariale. Le second accord VW va confirmer et renforcer la perte salariale. Néanmoins, les conflits qui apparaissent lors de la renégociation montrent que la communauté du travail est prête à soutenir le maintien de l'emploi activement. Tous les discours des représentants 
syndicaux le montrent : l'essentiel est de ne pas recourir aux licenciements économiques. De ce point de vue, la menace sur l'emploi est le premier facteur pour comprendre le comportement des syndicalistes. Chez BERGO, on s'inspire de la solidarité mise en œuvre chez VW. Les 700 licenciements annoncés sur un peu plus de 1300 salariés toucheraient presque un salarié sur deux. Ces licenciements annoncés par la direction ne deviennent « discutables » qu'après l'intervention du CE (représentations des salariés) qui applique une stratégie conflictuelle pour forcer la direction à négocier. Le fait que les négociations ont été menées par des non-syndiqués qui possèdent une légitimité incontestable dans l'entreprise souligne bien que, dans ce cas aussi, c'est la communauté de travail avec ses représentants qui voulaient obtenir un compromis sur le maintien de l'emploi.

\section{IV.5. La percée des régulations intermédiaires dans les relations professionnelles}

La réduction de la durée du travail de 35 heures à 28,8 heures (VW 1) et de 35 à 32 heures BERGO s'obtient dans des conditions particulières au regard du modèle des relations professionnelles décrits plus haut : la branche d'activité et la région constituent le terrain privilégié de la négociation du temps de travail. Avec la négociation de chez VW et de chez BERGO, l'entreprise devient le centre de ces négociations. Cette décentralisation de la négociation collective passe par des régulations intermédiaires (branche-entreprise). Il s'agit de nouveaux niveaux de négociation entre la branche et l'entreprise : chez Bergo un représentant non-syndiqué des salariés va interférer pour ramener les acteurs régionaux à la table de négociation, alors qu'il n'en a théoriquement pas le droit. Ce niveau de la négociation est nouveau, parce qu'il mélange entre acteurs locaux et acteurs de branche. L'exemple de VW et de ses conventions collectives «maisons » ont été quasiinstitutionalisée par la branche pour le partage du travail sous la pression des salariés dans les entreprises menacées. Ce sont des niveaux de régulation intermédiaires, c'est-à-dire « entre deux » et indépendant des deux niveaux.

Dans le cas de l'accord fondateur (VW 1), c'est bien l'existence d'une convention collective « maison » qui rend possible le partage du travail au niveau local. Chez BERGO où la négociation est en principe régionale, le CE obtient la prolongation « locale » de la convention collective régionale «maintien de l'emploi ». Ces conventions collectives sont la principale nouveauté des négociations aux niveau régional et permettent, par exemple dans la métallurgie, de déléguer la négociation du temps de travail à l'entreprise. En revanche, dans le cas de BERGO, la prolongation de cette convention collective est 
difficile à obtenir. Paradoxalement, cette convention collective de portée régionale n'est prolongée que pour le seul site de BERGO, ce qui indique l'existence de conflits intrasyndicaux concernant l'utilité de ces régulations intermédiaires et la légitimité des compromis. Il est plutôt surprenant de constater que les relations professionnelles allemandes rejoignent dans nos cas, d'une certaine manière les relations professionnels en France. La décentralisation des négociations et la concurrence syndicale locale autour du temps de travail peuvent en constituer des éléments.

\section{IV.6. Les temporalités sociales sont mises en causes, mais leur destruction n'est pas acquise}

La dynamique des négociations du temps de travail telle que nous l'avons présentée autour du cas de VW, nous semble indiquer un changement du paradigme de la négociation en Allemagne. Une dernière caractéristique de ce paradigme de la négociation concerne la mise en cause des temporalités sociales. Le partage flexibilisé du travail et des salaires semble dessiner pour l'avenir des durées plus courtes du travail sur l'année. Ces durées annuelles plus courtes sont paradoxalement compatibles avec des durées journalières et hebdomadaires plus longues du travail (au moins temporairement) et plus généralement avec une dégradation des conditions de travail dans leur ensemble: intensification, travail régulier de week-end, annualisation, voir pluri-annualisation.

En revanche, comme nous le montre l'accord VW 2, les demandes de flexibilité de la direction peuvent rencontrer l'hostilité des organisations syndicales et des salariés : dans ce cas, le conflit freine l'extension du travail le samedi. Chez BERGO aussi, les salariés ne sont pas toujours d'accord avec la mise en cause des temporalités sociales, notamment dans le cadre de l'annualisation: une durée maximale de 48 heures par semaine peut rallonger les temps de repos nécessaires pour récupérer et elle présente un risque pour la planification des activités extra-professionnelles.

Néanmoins, la fin d'un temps de travail et la mise en cause des temporalités sociales ne sont pas automatiques. Le conflit, mais aussi l'existence en France d'un référentiel temporel commun des organisations syndicales (montré au chapitre précédent) nous indique une matrice possible des comportements syndicaux à l'égard de cette mise en cause des temporalités sociales. 


\section{IV.7. Le partage du travail : une solution communautaire pour maintenir l'emploi?}

Nous partons ici de l'idée que le partage du travail en tant que tel refond la communauté de travail autour de la question de l'emploi. Plus exactement, en reprenant la terminologie de Max Weber (1921, 1980), nous pensons que le partage du travail constitue un processus de «communalisation ouverte» regroupant un ensemble des salariés menacés par le chômage. Par communalisation, Weber (1921, 1980, p. 21) entend : « une relation sociale lorsque l'attitude de l'agir social dans le cas individuel ou moyen se base sur un sentiment subjectif d'être ensemble \{Zusammengehörigkeit\} de manière affective ou traditionnelle $» .^{60}$ Ce processus de communalisation est « ouvert» et non fermé « quand la participation à l'agir social qui la constitue est orientée selon son sens \{Sinngehalt\} et n'est refusée, selon les ordres en vigueur, à personne pouvant et voulant s'y joindre. » (op. cité, p. 23). Dans ce sens, le partage du travail observé chez VW nous semble constituer un tel processus de « communalisation ouverte ». Nous pensons néanmoins que ce sentiment subjectif « d'être ensemble » ne doit pas être limité à l'activité affective ou traditionnelle », parce que nous voudrions rompre avec une lecture trop «classique » de la communauté présente dans le travaux de Tönnies (1912) ${ }^{61}$. Comme le remarque Zarifian (1996) à juste titre en s'appuyant sur un autre texte de Weber (1992), l'activité communautaire peut tout à fait obéir à une rationalité en finalité ou en valeur. Nous incluons donc ici l'extension de la définition restrictive de l'activité communautaire à ces deux autres types de l'agir dans la sociologie de Weber : l'agir en finalité (Zweckhandeln) et en valeur (Werthandeln). Cette position nous permet d'analyser l'effet d'un partage du travail autant par sa finalité que par les valeurs qu'il représente. La finalité de l'activité communautaire concerne bien le maintien de l'emploi : l'emploi de chacun est devenu plus «sûr» par le partage du travail. L'explication pourrait se situer d'abord au niveau des effets du partage sur le marché local ou interne du travail que représente l'entreprise. On pourrait penser qu'une fois le partage du travail réalisé, la position de ceux qui ont retiré par la réduction de la durée du travail des heures du marché « interne » du travail, donc la position des salariés dans les conditions du partage du travail situées ici du côté de l'offre du marché, se renforce tendanciellement par rapport à la demande de travail exprimée par la direction. La menace du chômage est relativement plus faible et donc la position globale des salariés et

60 Traduit par nos soins.

61 Notons ici l'importance des travaux fondateurs de Tönnies qui avait tenté de formuler le processus de l'émergence de la culture de la société moderne par le recours à deux catégories fondamentales que sont la «communauté » et la « sociét $\dagger^{\mathrm{a}}$ 
des syndicats dans la négociation se renforce. L'analogie du marché nous semble bien exprimer l'activité en « finalité » emploi des représentants des salariés.

Du côté de l'activité communautaire selon une rationalité en «valeurs », le sacrifice pourrait «souder» la communauté du travail autour de la question de l'emploi. Ce processus de solidarité interprété comme une «communalisation ouverte» crée un lien entre ceux qui peuvent et veulent adhérer au sens du partage : maintenir de l'emploi. L'engagement est local c'est-à-dire limité à l'unité concernée par l'accord. Les contractants se reconnaissent dans leurs engagements, mais les relations se transforment comme nous l'avons vu à l'occasion des conflits qui accompagnent la négociation de VW 2. Ce processus de communalisation qui est aussi un processus de rationalisation des temps de production dont les salariés sont conscients, crée une «dette» dans la relation entre employeurs et salariés. Les salariés ont concédé beaucoup à l'employeur pour maintenir l'emploi. Il devient d'autant plus «difficile» pour l'employeur de revenir plus tard sur cette arrangement légitime et de déconstruire par l'arrangement temporel la communauté qui s'est refondée autour de l'emploi : il faut des arguments nouveaux à la direction pour mettre en cause la garantie de l'emploi. La garantie de l'emploi constitue du point de vue des salariés la contrepartie des « sacrifices ». Cette garantie est de fait une « interdiction» de licenciements localement appliquée. Nous devons alors souligner que le « sacrifice» ne constitue pas ici une essence spirituelle ou une position idéologique visant à « dégrader » les conditions de travail des salariés dans le rapport qui les oppose aux employeurs. Au contraire, le partage du travail tel que nous l'avons défini, peut jouer sur les consciences des acteurs et en faveur du lien social : il participe à la reconstruction de la communauté au travail, préservent ses membres de son exclusion, et contribue à l'accueil de nouveaux salariés. Nous voyons des processus de solidarité qui renforcent la communauté de ceux qui ont partagé le travail. Malgré toutes les difficultés chez VW ou ailleurs, le sens subjectif attribué au partage représente bien les valeurs engagées du côté des salariés dans le compromis. 


\section{CHAPITRE VI \\ LA SUBSISTANCE ET LA FIN D'UNE NORME TEMPORELLE}




\section{Introduction}

L'éclatement du temps de travail et l'importance croissante de la défense de l'emploi montrent que les normes temporelles changent. La crise des normes temporelles se traduit par une pluralité de mondes temporels qui cohabitent : des temps différents composent des mondes de vie qui se démarquent les uns des autres selon les individus, selon leur statut d'emploi ou selon le contrat de travail.

L'objet de ce chapitre consiste à discuter l'ampleur de ce phénomène en ouvrant le regard sur d'autres pratiques temporelles. L'emploi à temps plein et à durée indéterminée est fortement concurrencé par d'autres statuts qui se développent dans le monde industrialisé. Peut-on comparer le temps de travail d'un technicien de l'aéronautique avec celui d'une caissière de supermarché? Il ne s'agit pas de mettre en cause la valeur centrale du travail pour les sociétés industrialisées, mais d'avancer l'idée de la fin d'une certaine conception du temps de travail

Le constat de l'écroulement d'une norme temporelle unique, telle qu'elle a été conçue en France au cours de 150 ans, serait incomplet, si nous ne prenions pas en compte l'évolution des statuts d'emplois et notamment le travail à temps partiel et l'emploi multiple. Ces emplois sont aussi des manières de vivre le temps qui mettent encore davantage en cause le caractère protecteur des règles temporelles. En concluant sur les chapitres précédants, cette dernière partie voudrait prolonger la réflexion sur la fin d'une norme. S'agit-il d'une évolution reposant sur des tendances contradictoires ou au contraire d'une évolution reposant sur des tendances parallèles? Comment les sociétés gèrent-elles cette brèche qui s'ouvre? N'assiste-t-on pas aujourd'hui à l'apparition d'une conception du temps de travail qui s'écarte d'une norme commune portant sur une durée journalière et hebdomadaire stable du travail?

\section{EFFONDREMENT D'UNE CONCEPTION DU TEMPS DE TRAVAIL}

A partir de 1830 nous assistons en France à une lente montée de la réglementation du temps de travail. On aurait pu penser que le temps de travail est une simple affaire de décret, le résultat mécanique de l'intervention de la puissance publique. Certes, le temps de travail est le premier domaine d'intervention de l'État, qui consacre l'Etat comme la puissance publique normative, le fondateur et le garant de l'ordre public social. En revanche on constate aujourd'hui une évolution de cette vision du temps de travail à la fois 
$\mathrm{du}$ point de vue des règles temporelles, mais aussi du point de vue des formes de régulations sociales qui sont mises en oeuvre.

\section{I.1. La perte de la centralité de la durée hebdomadaire du travail et l'avènement du temps des marchés}

L'évolution des normes se traduit depuis le début des années 1980 par une diversification et une complexification des codifications du temps de travail : les thèmes du temps de travail se multiplient. Il y a bien une perte de la centralité de la durée hebdomadaire du travail au profit d'autres composantes du temps de travail relatives à la répartition des temporalités journalières ou annuelles. Déjà en 1969 Grossin (p. 166) notait l'instabilité potentielle des structures temporelles, alors que peu de dispositifs touchant la flexibilité ${ }^{62}$ existaient alors: «On peut se demander, si les conditions futures de la production n'influeront pas davantage sur les caractéristiques du temps de travail autres que la durée : sur la structure des temps de travail internes, sur la stabilité hebdomadaire et annuelle des durées du travail, sur les distributions des temps de travail dans la journée et dans l'année ». Cette intuition de Grossin se trouve totalement confirmée aujourd'hui : il y a bien d'un côté une variabilité des temps de travail et de l'autre un déplacement de l'horizon temporel qui passe de la semaine à l'année, de l'année à la vie de travail toute entière de l'individu. Cette perte de la centralité de la durée hebdomadaire s'accompagne d'une variabilité des durées du travail qui va entrer en conflit avec les autres temporalités sociales.

Même si l'atomisation de la structure temporelle se traduit par une variété de configurations, il est clair que la structure temporelle du travail ne disparaît pas. Elle a deux caractéristiques majeures : elle se présente sous une grande variété de formes et fait perdre à la durée hebdomadaire son rôle central, puisqu'elle n'est plus l'univers de références de comptage et donc de disputes. Par voie de conséquence, c'est autour des nouvelles formes du temps de travail que vont se nouer des controverses et des conflits. L'analyse de la négociation du temps de travail révèle que le temps de travail ne donne plus lieu à des négociations concernant exclusivement les normes temporelles, c'est-à-dire la durée journalière et hebdomadaire, les repos et les congés. Le «temps des marchés », le « temps individualisé » et le «temps des machines » indiquent bien le tournant dans la négociation: les exigences de la production sont omniprésentes dans le temps de travail. Le temps « échangé » atteste que l'on ne traite plus de la durée du travail en elle-même : la

${ }^{62}$ Mises à part les heures supplémentaires. 
perte de la centralité de la durée hebdomadaire est consommée dans les pratiques. Nous proposons d'interpréter les résultats de la négociation collective, comme un retour renforcé sur la logique de la production, qui impose désormais sa loi sur la définition de la structure temporelle de travail. Cela donne une autre signification au temps de travail, dont on peut se demander s'il ne devient pas, soit le temps de la production, soit le temps de l'emploi, soit les deux. Le changement de perspective peut être interprété comme le passage vers des dispositifs orientés vers l'ordre de la production au détriment de dispositifs orientés vers le « bien-être » du salarié : ce passage s'accompagne-t-il d'un conflit entre ces deux logiques de production et du bien être ? En particulier le «temps des marchés » qui vise à adapter la disponibilité des salariés aux exigences du marché (clients, commandes) nous semble devenir une figure dominante de la flexibilité : les variations et la variabilité de la durée du travail au cours d'une période (pluri)annuelle semblent être la première exigence du cadrage temporel des activités professionnelles.

L'accord Volkswagen, bien qu'il réduise la durée du travail à 28,8 heures par semaine, poursuit cette logique : il permet à l'entreprise de conserver dans des périodes de «sousactivité » une main-d'oeuvre nombreuse (1994) qu'on peut mobiliser de manière instantanée dans des périodes de «surcharge » (1996). Une nouvelle baisse d'activité prévue se traduit de nouveau par une baisse du temps de travail effectif (1999) : la durée du travail devient une moyenne annuelle qui sert de référence pour le paiement des salaires, mais qui laisse toute la liberté à l'employeur de réagir face aux exigences du marché. En France et dans le contexte de la récente loi «Aubry», les 35 heures remettent en jeu la durée hebdomadaire du travail et on pourrait penser au contraire à une tendance qui vise à retrouver la règle protectrice autour de la durée légale. Autrement dit, il pourrait s'agir d'un recentrage des dimensions du temps de travail sur la durée hebdomadaire. Mais au regard de l'évolution de la négociation collective telle que nous l'avons présentée, la nouvelle loi doit s'analyser au travers des pratiques : celles-ci semblent enfermées dans l'échange entre la réduction de la durée du travail d'un côté et les temps des marchés de l'autre côté, sur fond d'action pour l'emploi. Par ailleurs les scénarios de la réduction de la durée du travail selon les secteurs et la taille d'entreprise risquent d'être fort hétérogènes. Cette hétérogénéité est avant tout liée à la pluralité des acteurs et de leur conceptions du temps de travail. Quel prix doit-on payer pour défendre l'emploi dans l'entreprise? Les organisations syndicales peuvent-elles accepter que la protection de l'emploi l'emporte sur la qualité de la vie au travail? 


\subsection{Des représentations diverses sur fond commun de résistances}

Les dynamiques et les contradictions de l'action syndicale en France recouvrent un ensemble de représentations du temps que nous avons tenté d'analyser autour du terme de « référentiel négociatoire ». Nous en avons distingué deux : le référentiel singulier et le référentiel commun. Le référentiel commun est composé de valeurs que partagent les organisations syndicales : du temps pour soi et pour tous, la stabilité des repères temporels et la maîtrise de l'emploi du temps. Les 150 ans de réglementation du temps de travail et, en particulier, le compromis social issu des trente glorieuses, ont contribué à l'existence de ces valeurs communes entre syndicats. En revanche le référentiel singulier est plus sensible au changement et s'ajuste à court terme : il s'agit d'un référentiel de situation qui peut évoluer sous la force de choses. Ce référentiel singulier ou spécifique de chaque organisation syndicale est construit historiquement et se rapporte à une situation. Ce référentiel «cognitif» incorpore toute la mémoire et les valeurs transmises. L'analyse comparée de ces référentiels montre leur spécificité. Néanmoins des actions collectives locales ou ciblées selon les thèmes et des alliances restent possibles. L'analyse des arrangements temporels montre la complexité de la négociation, mais aussi que le temps de travail n'est plus traité pour lui-même, mais pour autre chose ; il sert d'outil de gestion de l'emploi. Autrement dit, le temps de travail ne serait plus qu'un moyen de marchandage pour obtenir autre chose, notamment le maintien de l'emploi, comme si l'on glissait du temps de travail au temps de l'emploi. Il nous faut nuancer cette affirmation.

L'explicitation de ce qu'il y a de commun dans les représentations des organisations syndicales des salariés, nous a permis de montrer que le temps de travail doit être, (a) repensé dans le sens d'un allégement, car la pression temporelle est trop forte, (b) articulé avec les autres temporalités sociales, dont celle de la famille, pour limiter les conflits temporels, même si les raisons évoquées varient d'un syndicat à l'autre, (c) relié avec la question du maintien de l'emploi, même s'il faut se soumettre aux exigences de la flexibilité, enfin (d) un moyen pour développer plus de solidarité entre ceux qui ont un emploi et ceux qui en sont, plus ou moins durablement privés. Autrement dit, on est loin d'une acceptation, passive ou active, de la logique de la production; mais la manière d'orienter l'action syndicale vers la logique de la vie quotidienne des salariés change: l'action syndicale s'oriente, de plus en plus, vers des logiques de compromis, dès que l'entreprise ou l'emploi sont menacés. L'incertitude du lendemain, l'insécurité sur les conditions matérielles d'existence amènent une plus grande variabilité des positions, un 
registre de réponses plus étendu, et souvent un résultat de la négociation incertain et fragile.

\section{LA FRAGMENTATION DE L'EMPLOI : LE TEMPS REDUIT OU RALLONGE}

La variabilité des règles temporelles définies dans des contextes locaux et le glissement de ces règles, vers la flexibilité de la production et vers l'emploi, indiquent une diversification des mondes temporels. Ces mondes cohabitent parfois à l'intérieur d'un même atelier. Tous les salariés ne sont pas exposés aux heures supplémentaires ou au travail de nuit. En revanche, une inversion des priorités du cadrage temporel nous semble incontestable. Pour appuyer cette position nous voudrions aborder par une perspective différente les rapports entre le temps et la société. L'évolution vers des emplois à temps « réduit », ou vers des emplois secondaires voir multiples, nous indiquent que le temps de travail est pour une partie des salariés un temps fragmenté : soit parce qu'il ne correspond que «partiellement» aux temps professionnels conventionnels, soit parce qu'un temps plein ne suffit plus à subvenir aux besoins de survie. Dans les deux cas les temps professionnels sont fragmentés. Cette évolution dans laquelle l'individu est confronté à un temps « en portions » décrit une autre manière de voir les règles de protection qui ont constitué le coeur de la conception du temps de travail qui a prévalu jusqu'à maintenant.

\section{II.1. La fragmentation directe : le temps partiel}

Le travail à temps partiel regroupe les salariés dont le contrat prévoit une durée hebdomadaire d'au moins $20 \%$ inférieure à la durée légale du travail. Mais pour $58 \%$ d'entre eux, elle est inférieure à 20 heures par semaine; pour la moitié de ces $58 \%$, la durée contractuelle est de moins de 15 heures hebdomadaire (INSEE-DARES, 1995, p. 56). Observons la progression très importante du travail à temps partiel. Selon les données fournies par l'enquête « emploi » (op. cité) ${ }^{63}$, la proportion des salariés travaillant à temps partiel est passée de 8,5\% en 1984 à $14,7 \%$ en 1994 . Notons que seulement un homme sur 20 est concerné par le travail à temps partiel (4,5\%), alors que plus d'une femme sur 4 $(27,8 \%)$ se trouve dans cette situation. Le taux du travail à temps partiel en France est proche de la moyenne européenne (16,2\% contre 16,7\% en moyenne). En revanche, nous pouvons constater que les écarts à la moyenne sont très variables. Le taux des Pays-Bas se situe à un niveau deux fois plus élevé : $37,4 \%$ des emplois sont des emplois à temps partiel ; en revanche au Portugal et en Grèce, moins de 5\% des emplois sont à temps

${ }^{63}$ op. cité 
partiel. D'autres enquêtes montrent que le salarié à temps partiel est plus touché par l'instabilité de la localisation des périodes de travail que le salarié à temps complet. Ce qui confirmerait des liens observés entre les processus de délocalisation et d'individualisation. Ainsi en 1984, la part des salariés qui avait les mêmes horaires au jour le jour est réduit pour le temps partiel $(50,7 \%)$ par rapport au temps complet $(59,5 \%)^{64}$.

Selon les pays européens et de manière variable, l'individualisation contribue à la division sexuée du travail professionnel. Dans l'Europe des 15 en 1995, une femme salariée sur trois travaille à temps partiel $(32 \%)$, contre un homme sur vingt $(4,5 \%)$. Cependant la proportion des femmes à temps partiel varie sensiblement d'un pays à l'autre. Ainsi, aux Pays-Bas plus de 2/3 des femmes salariées travaillent à temps partiel (67\%); en revanche, en Grèce seulement 5,5\% des femmes salariées sont à temps partiel. Pour ces deux derniers pays, nous pouvons constater que moins les femmes travaillent à temps partiel, moins le taux global du travail à temps partiel est important. Pour interpréter cette différenciation du temps de travail, Bloch-London, Bué et Coutrot (1996) considèrent alors que le travail à temps partiel pourrait être vu comme une variante « féminine » du partage du travail : du «féminin singulier» (contrat de travail) au «masculin pluriel» (accord collectif). Un bilan montre que le travail à temps partiel est inégalement développé en Europe, mais certaines caractéristiques collectives se retrouvent, (Maruani, 1996). En effet, le temps partiel souligne plus particulièrement la division sexuée du travail : «Là où il se développe, le travail à temps partiel accentue en effet la concentration des emplois féminins en les cantonnant dans un nombre encore plus réduit de professions et de qualifications », (op. cité p. 184). La fragmentation directe du temps de travail par le travail temps partiel possède donc des caractéristiques qui dépassent l'individu dans le sens où des groupes de salariés deviennent les destinataires d'une même règle ou d'une même place dans une hiérarchie des mondes temporels. On peut penser que la règle sociale qui distribue la population sur des emplois à temps partiel est une manière de confirmer, voire d'accentuer la division sexuée du travail.

\section{II.2. La fragmentation indirecte : la baisse des salaires}

Ces quelques exemples de l'apparition massive du travail à temps partiel nous montrent le terreau sur lequel peut apparaître un «temps vécu de la subsistance ». Entre l'emploi à temps plein et le chômage, le travail à temps réduit est une configuration

${ }^{64}$ op. cité 
intermédiaire de plus en plus observée. Dans le cas du temps partiel, le salarié est rémunéré pour un temps « court », et trop court, parfois, pour subvenir à ses besoins. Se pose alors la question du complément financier qui devra être obtenu notamment par des activités complémentaires rémunérées et qui plonge l'individu dans la pluri-activité. Ce même processus de recours à des activités complémentaires peut aussi porter sur le travail à plein temps. Dans le cas d'une baisse sensible des salaires dans une entreprise, il faudra consacrer du temps dans un emploi complémentaire pour gagner sa vie. Dans les deux cas, nous sommes en présence d'une pluri-activité rémunérée pouvant conduire à l'occupation de plusieurs emplois.

Nous voudrions interpréter cette situation en termes de temps de la subsistance que l'on peut définir comme le temps destiné à récolter «l'ensemble des vivres et objets au moyen desquels on subsiste $»^{65}$.

Au travers d'une brève étude effectuée en Russie ${ }^{66}$ nous avons utilisé la notion de « temps de la subsistance ». La caractéristique de ce temps de la subsistance est celui-ci de sa fragmentation, vue sous l'angle de l'emploi. Deux salariés nous parlent de leur situation professionnelle. Tous les deux doivent combiner des activités professionnelles suite à une chute des salaires.

« Je travaille en trois endroits différents. Ma première place est à l'université. Mais, malheureusement, on ne nous paye pas parce que la situation de notre pays est très grave, c'est-à-dire nous devons attendre des mois pour recevoir le salaire et c'est [700 francs] par mois environ. Après, je travaille dans un autre institut pour donner des cours et enfin le troisième emploi, c'est dans un collège. [...] Au cours de l'année, ça change. Au mois de janvier, je n'ai presque aucune conférence parce que les étudiants passent les examens. Au mois de décembre, j'ai beaucoup de cours et du travail supplémentaire. Evidemment, au mois de janvier, je ne gagne que le salaire de mon travail principal » (enseignante). « Je suis pris dans les engrenages, il faut faire le travail au service principal, c'est-à-dire dans l'armée, et il faut enseigner quelque part pour gagner de l'argent pour nourrir la famille » (un enseignant).

La situation est différente de celle précédemment évoquée à propos du travail à temps partiel. La fragmentation de l'emploi est indirecte. Ici les salaires sont insuffisants aux

\footnotetext{
${ }^{65}$ Larousse (1988, p. 1801).

${ }^{66}$ Il s'agit d'une enquête monographique dans trois entreprises de secteurs différents (électronique, nettoyage industriel et service de documentation). Nous avons mené une vingtaine d'entretiens en collaboration avec Igor Massalkov (Université de Lomonossov, Moscou) auprès de responsables de directions et des salariés pour comprendre les pratiques temporelles en Russie.
} 
yeux des salariés et le temps de travail de l'entreprise ne se modifie pas. Il s'agit pour des salariés à plein temps de trouver d'autres sources de rémunération en occupant des emplois complémentaires. Cette nécessité d'avoir des activités rémunérées supplémentaires semble être importante pour les retraités, comme le souligne cette employée : « ce n’est pas un secret qu'il est impossible de vivre avec cette somme d'argent ».

Mais ce temps de la subsistance se poursuit aussi dans la vie en dehors des activités professionnelles rémunérées. Pour pouvoir disposer d'un temps suffisant pour des activités de travail non rémunérées comme le jardinage, les postes longs et des semaines comprimées sont préférés par certains salariés : «Parce qu'après le travail en 1 poste, ils ont presque 3 jours libres. Parce qu'ils sont chez eux 2 jours, et le troisième jour, ils ne commencent à travailler que le soir. C'est très pratique pour ceux qui ont des datchas. Vous comprenez? Vous savez bien que maintenant chez tout le monde, c'est comme ça : on mange ce qu'on a planté et cueilli. Voilà. Mais, bien sûr, on a aussi besoin d'un régime de travail plus doux » (une opératrice d'une entreprise électronique).

Pour d'autres, la pluriactivité tournée vers la subsistance, concerne le développement d'activités non rémunérées pour terminer la maison de famille : « J'utilise mes congés pour travailler dans la banlieue, ma belle-mère a une maison et un petit terrain qu'on appelle chez nous datcha, la maison n'est pas terminée, je travaille là-bas comme menuisier, charpentier », (un enseignant).

La conséquence de ces pratiques temporelles est la diminution du temps libre ou des loisirs. Il disparaît complètement derrière les besoins de subsistance, comme l'indique cet enseignant.

« Je n'ai pas de loisirs. Mon loisir à Moscou, c'est regarder un film à la télé, aller chez un copain, mais je ne peux pas aller au restaurant, parce que je n'ai pas suffisamment d'argent». Une opératrice dans une entreprise électronique fait plutôt un lien entre l'absence de temps libre et les effets négatifs du travail sur la santé.

« Les symptômes de tuberculose, nous les voyons chaque jour. Il n’y a plus de temps libre. La fatigue s'accumule, surtout après le travail de nuit : 2 postes de nuit, chacun sur 12 heures. Le premier jour libre tu dors, le deuxième tu récupères de la fatigue. Partout c'est comme ça, peut-être. Personne ne le conteste. La famille s'est habituée, comme on dit. Maintenant il y a beaucoup de problèmes avec le temps libre. Il me manque. A mon âge, je m'occupe juste des travaux domestiques, c'est tout. En général, le travail ici est très scrupuleux. Beaucoup d'hommes ne le supportent pas », (une opératrice). 
En somme, ce temps de la subsistance se caractérise par la pluriactivité et l'emploi multiple, Mais aussi par l'émergence d'un temps consacré à la production d'aliments, au bricolage, ce qui se traduit par la réduction du temps libre pour tous. Un vigile de magasin décrit la cohérence et le vécu difficile de ce temps de la subsistance : «Essentiellement, c'est la course à s'essouffler, la quête des moyens pour exister. Avant, j'avais une activité permanente que j'aimais. Maintenant, je suis obligé de m'occuper de ce je n'aime pas, de ce qui n'est pas stable. Tout ça pour exister. C'est ainsi que je passe mes jours, mes semaines. C'est toujours la course, la quête, la débrouillardise. Les jours et les semaines peuvent être différents. Mon métier, c'est bâtisseur et je cherche à être embauché en soustraitance. Je propose mes services à des gens et des organisations différentes. Il s'agit de trouver les matériaux de construction par exemple. Et pratiquement, c'est tous les jours comme ça. Ce que j'aime, ce sont les travaux dans le jardin potager de ma datcha. Ce sont mes activités préférées. Dans une certaine mesure, ça me donne à manger. Le travail luimême et ensuite ce qu'il m'apporte ne me plaît pas. Je suis bâtisseur et pour le moment malheureusement il n'y a pas de travail de ce genre, il n'y a pas de commandes. Je suis pessimiste, tout simplement. Comme beaucoup d'autres à l'heure actuelle» .

Ces quelques exemples illustrent le fait que pour un ensemble de personnes le temps de travail, c'est le temps de la subsistance. En effet les activités de travail rémunérées sont parfois complétées par l'occupation d'un autre emploi rémunéré pour disposer d'un revenu suffisant. De même les activités de jardinage constituent une possibilité pour certains d'avoir des produits qu'ils ne pourraient s'acheter. On le voit, la pluriactivité se traduit par l'extension des activités de travail à la sphère domestique et l'occupation de plusieurs emplois fractionnés.

\section{EMPLOIS MULTIPLES : LE TEMPS ADDITIONNE}

L'individualisation du temps de travail et de sa division « en portions » sous forme de temps partiel peut provoquer aussi le cumul des activités professionnelles. Ce cumul traduit d'abord l'insuffisance d'une seule activité ou d'un seul métier pour pouvoir survivre. Même si le recours extensif au travail à temps partiel est relativement récent, il faut souligner, contrairement à une idée reçue, que nos sociétés industrialisées subissent depuis longtemps cette tendance à un cumul des activités. L'emploi multiple en début et fin de ce siècle fait partie de la vie quotidienne d'une partie des salariés. 


\section{III.1. L'emploi multiple en début de siècle}

Dans un texte qui s'intitule «Sur la Psychophysique du travail industriel » (1908) Max Weber $(1924,1988)$ s'efforce de montrer la nécessité d'intégrer dans l'analyse du travail industriel des questions touchant au temps de travail. Le temps de travail apparaît dans ce texte comme fortement tributaire du contexte social et local. Il est en ce début de siècle d'abord un temps de travail subjectif lié aux trajectoires, mais aussi lié aux conditions techniques et sociales de l'exercice du travail. Ce temps de travail est véritablement lié à la société toute entière. La question de la crise des métiers, et des durées du travail variables laisse apparaître la configuration d'un temps de la subsistance : celui-ci renvoie à la perte des heures de travail et des salaires correspondants pour certaines activités et en particulier lors des crises de chutes des commandes. Le temps et l'emploi sont ainsi fragmentés selon les activités, ou autrement dit : les temps de travail s'additionnent. L'individu est alors contraint de chercher des heures de travail en plus ou de subir le sous-emploi.

L'exemple de cette ouvrière du textile montre que la conjoncture en 1908 réduit son activité de moitié, parce qu'un de ses multiples métiers est particulièrement touché par la crise : «Le recul de son temps de travail en septembre 1908 s'explique par le fait que la dépression et la réduction de l'activité de l'entreprise de ce mois avaient un effet particulier sur l'activité de cette ouvrière. (...) Elle n'a été employée au rendement que pendant 15 jours (des 26 jours de travail de ce mois) ; elle avait donc souffert deux fois plus de la réduction de l'activité que les autres ouvriers, dans la mesure où elle ne trouvait du travail que sur 4 jours de la semaine et le temps nécessaire pour une des activités avait diminué d'un cinquième. » (op. cité, p. 220).

La menace du sous-emploi par la crise pèse sur certaines activités professionnelles. En revanche, il faut situer cette perte d'activité dans la menace globale du chômage qui a selon Weber une influence considérable en tant que moyen d'action patronal pour augmenter la rentabilité des ouvriers. «Le moyen de l'accroissement de la capacité et de la volonté de travail des ouvriers est, d'une part, la prime directe affectant (...) le système de rémunération : le type le plus simple est le salaire au rendement pur. D'autre part, l'augmentation de la rentabilité est obtenue par la sélection, autrement dit par l'exclusion des ouvriers les moins capables ou les moins volontaires au travail. La possibilité de l'application du dernier moyen, du fouet menaçant du chômage, contribue au moins ${ }^{67}$ autant au développement de la capacité de travail des ouvriers,(...) que la dépendance du niveau du salaire par le rendement » (op. cité, p. 127).

${ }^{67}$ souligné par Weber 
La crise du temps de travail est donc vue par Weber au travers la menace du chômage non seulement comme un effet économique sur l'emploi, mais aussi comme l'exercice d'un moyen de contrôle social sur le travail des ouvriers. Le revers de la médaille est que l'employeur court dans la crise le risque, d'observer une dégradation du travail des ouvriers, donc une perte de sa rentabilité : l'industrie textile et l'industrie de machine d'outil montrent dans la crise une réduction du temps de travail accompagné d'un travail moins régulier (p. 154).

On peut penser que la menace du chômage et la fragmentation de l'emploi ont joué un rôle dans l'émergence du temps industriel. En revanche, nous devons aller plus loin. L'emploi divisé en métiers du début du siècle n'a pas grande chose à voir avec un temps de travail « éclaté » ou un temps de «l'emploi partagé » tel que nous l'avons présenté plus haut. Ce temps de la subsistance est au contraire caractérisé par son individualité, par la diminution de l'effet « protecteur » des règles en particulier sur l'emploi, mais aussi par les contraintes liées à la subsistance des individus. Dans cette configuration, c'est la recherche des «heures» et le rallongement du temps de travail individuel qui est au centre des préoccupations et non sa diminution. Les temps de travail s'additionnent.

\section{III.2. Les « contingent workers 》 et le cumul des emplois}

Aux Etats-Unis, on appelle « contingent workers » les travailleurs soumis à une flexibilité de leur statut d'emploi : les estimations sur leur importance réelle varient de $13 \%$ à plus de $30 \%$ de l'ensemble des salariés, mais il y a une certitude sur leur importance grandissante (Crittenden, 1994). Parmi ces «contingent workers» le temps partiel joue un rôle primordial. Il est devenu la configuration «typique » de la création d'emplois pendant les dernières années. En ce qui concerne la dynamique de sa diffusion, on peut dire qu'il est deux fois plus important dans la création de nouveaux emplois au cours de la période de 1990 à 1993. Presque deux emplois sur trois créés sont des emplois à temps partiel. ${ }^{68}$

La codification administrative du temps partiel et de l'emploi multiple pose ici un problème d'interprétation. Les chiffres sur le temps partiel en tant que catégorie des « contingent workers » ont tendance à sous-estimer l'ampleur du phénomène. Deux remarques peuvent être formulées quant à la construction de cette catégorie. D'abord, notons que la norme du temps partiel est définie par un travail d'une durée de moins de 35 heures par semaine. Elle inclut aussi bien le contrat à 10 heures et celui à 34 heures. En

${ }^{68}$ Source : US Bureau of Labor Statistics, bulletin 2307 
revanche, quand on occupe 2 emplois de 18 heures chacun, on est considéré comme travailleur à plein temps, alors que le véritable travail à plein temps dépasse largement les 40 heures et bénéficie des conditions de salaire nettement plus favorables. Ensuite, une bonne partie des salariés à emplois multiples travaillent de manière non-déclarée dans l'économie parallèle et ils ne peuvent pas être recensés par les méthodes du micro-census (Owen, 1989). Ces salariés échappent alors aux statistiques, bien qu'ils alimentent le groupe des salariés à emplois multiples. Ce sont jusqu'à $75 \%$ des salariés qui ont un emploi à plein temps, plus un mi-temps. Malgré l'occupation supplémentaire d'un travail non-déclaré, ils sont comptés dans la catégorie de travail à plein temps, dont on néglige le temps partiel supplémentaire (Rosenberg, 1993). Le nombre d'emplois à temps partiel est donc systématiquement sous-estimé. Que peut-on dire sur la durée du travail de ces salariés à emplois multiples ?

La plupart de ces salariés à emplois multiples travaillent beaucoup plus qu'un salarié à plein temps. Cette évolution, si elle s'amplifie dans l'avenir, mettrait en doute en effet la polarisation du salariat américain autour des durées courtes et longues. En effet, la sommation des durées courtes, fait des durées globales plus longues pour les individus, auxquelles il faudrait ajouter les temps de transport d'un emploi à l'autre. D'après Yates (1994), en 1989, la durée moyenne pour un salarié qui détient plusieurs emplois est d'environ 53 heures hebdomadaires pour les hommes et de 47,1 heures pour les femmes ; de plus $14 \%$ des salariés de cette catégorie travaillent plus de 70 heures par semaine, ce qui équivaut, selon la définition officielle, à deux emplois à temps plein.

Si l'on prend en compte le fait que la norme des 40 heures (plus quelques heures supplémentaires) est toujours en vigueur pour une bonne partie des salariés à plein temps ${ }^{69}$, on ne peut que constater les clivages croissants entre les «contingent workers » et les salariés à durée de travail «normale». Ici aussi divers mondes temporels cohabitent. Quelques exemples permettent d'illustrer les contraintes de la vie professionnelle pour les temps partiels : les salariés à temps partiel ne gagnent en moyenne que $62,3 \%$ du taux horaire d'un plein temps. De même, seulement $15 \%$ de ces salariés obtiennent une assurance médicale payée par l'employeur, contre $65 \%$ des salariés à plein temps. Seuls $10 \%$ des temps partiels bénéficient d'une retraite patronale contre $46 \%$ des pleins temps (Crittenden, 1994). Le travail à temps partiel concerne environ $20 \%$ de l'ensemble des emplois. Selon Tilly (1991), les employeurs continuent à créer des emplois à temps partiel,

\footnotetext{
${ }^{69}$ Rosenberg (1993) avance le chiffre de 62,2 \% des salariés qui sont à plein temps en 1985.
} 
bien que les salariés n'en veuillent pas : ces emplois sont caractérisés par une faible qualification et le turn-over est élevé. L'extension du temps partiel est souvent une stratégie de gestion de l'emploi pour de grandes entreprises. Lors d'un conflit de travail en 1997, on constate que le service de courrier United Parcel Systems emploie environ 176.000 salariés à temps partiel avec des contrats qui ne portent souvent que sur quelques heures. Ces contrats regroupent $57 \%$ des salariés de l'entreprise. Le taux horaire du temps partiel atteint seulement $50 \%$ du taux en vigueur pour ceux qui sont à plein temps. ${ }^{70}$

L'appellation de cette catégorie de salariés de contingent (occasionnel, éventuel, incertain ou accidentel) exprime la différence avec l'emploi régulier et stable. Elle décrit du point de vue de l'emploi «unique » et à plein temps, l'écart par rapport à une occupation permanente dans un espace productif donné. Il est significatif ici que la mesure du temps de travail ne s'applique pas à l'espace productif occupé, mais à l'individu. De ce point de vue, deux temps partiels font un plein temps. De plus, l'appellation de «contingent worker » est ambiguë, puisqu'elle sous-entend une certaine marginalité du phénomène et une absence de règles et de régularités, voire le fait du hasard : cette appellation suggère des temps de travail courts. Le nombre important de personnes concernées par ce type d'emploi et les durées du travail réellement exercées mettent aujourd'hui en question cette vision.

De plus au niveau du temps partiel auquel on avait aussi attribué ce pouvoir de faciliter la concordance des temps, le résultat ne correspond guère aux attentes. «En ce moment la grande majorité des emplois à temps partiel sont mal payés, avec peu de perspectives de carrière et souvent sans intéressements » $\left(\right.$ Schor, 1991). ${ }^{71}$ La notion du temps « choisi » est ici relativisée par le contenu du choix. L'exemple d'une employée de la Bank of America qui y travaille depuis 14 ans le montre. Elle avait le choix de réduire sa semaine de travail de 30 heures à 19 heures par semaine, sans compensation financière, ou de quitter l'entreprise avec une prime forfaitaire. Elle a décidé de rester dans l'entreprise. Elle occupe maintenant un emploi secondaire dans un magasin pour pouvoir assurer sa survie. Ce choix a été proposé en 1993 à des milliers d'employés de la Bank of America. Au total, seulement $19 \%$ des emplois sont restés des emplois à plein temps (Crittenden, 1994).

La pression pour «choisir» fait basculer l'individu de salarié permanent à salarié temporaire (Pialoux \& Beaud, 1993).Une fois précipité dans la catégorie de ceux qui

\footnotetext{
${ }^{70}$ Der Spiegel (1997), traduit par nos soins.

${ }^{71}$ traduit par nos soins
} 
n'occupent que temporairement l'espace productif, les conditions de travail risquent de se détériorer: la baisse du salaire, la carrière freinée, l'incertitude concernant l'assurance maladie et la retraite en sont des exemples. Paradoxalement, comme nous l'avons vu dans le cas de l'emploi multiple, le salarié concerné doit s'habituer à des durées de travail globalement plus longues.

\section{TEMPS DE LA SUBSISTANCE OU TEMPS PROTEGE?}

De toutes nos observations, nous voudrions en donner une interprétation d'ensemble provisoire. Trois évolutions analysées se combinent pour décrire une tendance. Il ne s'agit pas de la fin du travail, mais de la fin d'un mode de structuration des activités professionnelles et non professionnelles.

D'abord, nous avons relevé une autre façon de réglementer le temps de travail qui donne lieu à d'autres figures du temps de travail. Celles-ci rangent les temps de travail définitivement dans la pluralité et l'éclatement de ses formes. Néanmoins, cette déconstruction donne lieu à une restructuration complexe de l'ordre temporel. Cet ordre est local et tourné vers la production.

Ensuite on passe du temps de travail au temps de l'emploi. Ce passage nous semble significatif de la négociation du temps de travail : il varie en fonction des trajectoires et des situations. Nous passons d'une conception du temps centrée sur la protection de la santé et du bien-être des individus, à une conception basée sur la protection de l'emploi.

Enfin, dans certains cas, on passe du temps de travail au temps de la subsistance. Il s'agit d'un temps dirigé vers la recherche des « heures » de travail rémunéré, aboutissant à des emplois fragmentés et combinés en vue d'assurer la subsistance, à défaut de trouver mieux et en attendant que la situation change.

S'agit-il ici de voies alternatives ou de scénarios probables? Nous résumons ces trois évolutions simultanées, pour en durcir les traits, en deux alternatives qui s'opposent de manière caricaturale.

Le premier scénario d'une évolution du temps de travail serait basé plutôt sur le maintien d'un échange collectif en vue de réglementer la structure temporelle. Au centre nous voyons la convention «collective » qui, tout en acceptant une dégradation potentielle des conditions de vie dont les acteurs déterminent l'ampleur, vise à maintenir un « minimum » de repères temporels. Il s'agit aussi de permettre un minimum de dispositifs de protection 
du salarié, tout en interdisant le licenciement économique. Ce premier scénario met au centre l'emploi qui est échange selon les rapports de force en vigueur contre une acceptation de travailler autrement et souvent plus difficilement.

Un second scénario favoriserait plutôt la négociation de «face à face » entre le salarié et son employeur, qui déterminerait « librement» la durée, les horaires et le prix du travail. Dans cette situation où les règles temporelles sont à dominante contractuelle et non conventionnelle, on pourrait penser à une massification des emplois à temps fragmentés, réduits et additionnés. Sans protection légale, ni conventionnelle de son statut et de sa survie, le salarié ne pourrait compter que sur son poids individuel dans un marché du travail dont il ne contrôle pas les règles. Les situations observées dans ce chapitre seraient davantage tournées vers cette seconde alternative, tout en intégrant des éléments de protection notamment légale dans cette tendance. La première alternative sous le signe de l'emploi pourrait être plus proche des cas observés en France et en Allemagne, tout en maintenant une diversité de mondes temporels qui n'exclut pas «l'exclusion», ni la fragmentation de l'emploi.

Les analyses effectuées en France et en Allemagne pourraient indiquer une préoccupation commune de défense de l'emploi et de procéder à une flexiblisation «contrôlée » des durée du travail. Autour des cas analysés, nous pensons pouvoir interpréter le partage du travail comme une norme alternative au temps de la subsistance dans lequel pourraient tomber de nombreux salariés. Cette position n'exclut pas les conflits : tout au contraire, les conflits observés dans l'entreprise seront structurés autour des caractéristiques du partage (emploi, flexibilité et perte salariale). Les effets de ces «deals» sur la santé du salarié restent à mesurer : la flexibilité et l'intensification discontinue du travail dégradent dans bien de cas les conditions de vie au travail. Certes les durées conventionnelles du travail diminuent. En revanche, l'alternance des périodes de travail liées aux fluctuations des marchés produit des «pics » et des périodes de pointe, liées aux temps des marchés. Cette intensification discontinue du travail se traduit par des rallongements de la journée ou de la semaine de travail bien au-delà des conventions antérieures. Cette situation d'une intensification de certaines périodes de travail en échange d'un allégement du travail dans d'autres périodes n'est pas sans risques pour la santé des salariés.

La généralisation difficile de la solution Volkswagen du fait de la perte salariale élevée, notamment pour le secteur d'automobile montre l'importance de ces conflits. Observons que les thèmes du partage (flexibilité, emploi et baisse des salaires) sont très présents en 
France. L'Etat se voit jouer un rôle moteur dans la mise en oeuvre de la réduction de la durée du travail. Les politiques publiques en témoignent depuis des années. L'activité négociatoire est en forte expansion, même si les résultats l'emploi ne correspondent guère aux attentes. Les prochaines crises ne pourraient-elles pas contraindre les entreprises en Europe de converger sur des solutions temporelles visant le maintien de l'emploi? L'intervention des acteurs de l'Union Européenne pourrait jouer un rôle plus important dans la fixation de règles communes. Mais l'analyse de la négociation du temps de travail montre aussi que les espoirs projetés sur la force des règles légales ne se réalisent pas toujours. La régulation du processus qui aboutit à des arrangements temporels diversifiés semble contrôlée d'en haut, car l'action collective produite par l'ouverture de l'espace de négociation a produit sa fermeture. Les accords du partage flexibilisé en France et en Allemagne en sont une illustration. L'espace de négociation se retrouve reverrouillée sur le triangle flexibilité, emploi, durée du travail. De plus, de cet espace naissent de nouvelles rationalisations du temps de travail principalement liées au mouvement des marchés : l'annualisation du temps de travail, la gestion pluriannuelle de ce temps à l'aide des comptes épargne temps ou encore la gestion du travail sur la vie entière. Ceci montre bien, qu'il s'agit d'un processus dont les conséquences sont importantes sur la vie sociale. Ni le temps, ni le travail n'échappent à ce processus de rationalisation dont on ne peut pas dire avec certitude comment il évolue : c'est aux acteurs de la négociation d'en décider. 



\section{BIBLIOGRAPHIE}

- Alaluf, M. (1986). Le temps de labeur : formation, emploi et qualification en sociologie du travail. Bruxelles : Éditions de l'Université de Bruxelles.

- Ansart, P. (1991). Les inventeurs des rythmes sociaux, Cah. Intern. de Sociologie, XCI, 229-240.

- Autrand, A. (1988). Le mouvement syndical français et le temps de travail : des stratégies différenciées ", Revue française des Affaires Sociales, 1 .

- Benguigui, G., Griset A., \& Montjardet D. (1978). La fonction d'encadrement. Paris : La Documentation Française.

- Bloch-London, C., Bué, J., \& Coutrot T. (1996). Politiques de l'emploi : masculin pluriel ou féminin singulier. In : H. Hirata \& D. Senotier (Eds.), Femmes et partage du travail (pp. 163-176). Paris : Éditions Syros.

- Bloch-London, C. \& Marchand, O. (1990). Les enjeux de la durée du travail. Economie et Statistique, 231, 19-32.

- Boisard P. (1995). Durées et horaires de travail de travail dans les pays de l'Union européenne. Communication présentée au Sixième Séminaire International sur le Temps de Travail (SITT). Blankenberge, Belgique, décembre.

- Bosch, G. \& Lallement, M. (1991). La négociation collective sur le temps de travail en France et en Allemagne, Travail et Emploi, 49, 31-49.

- Bouffartigue, P. \& Boccino, M. (1998).Travailler sans compter son temps? Les cadres et le temps de travail. Travail et Emploi, 74, 37-50.

- Boulin, J.-Y. (1993). L’organisation sociale du temps. In : J.-Y. Boulin, G. Cette, D. Taddei (Eds.), Le temps de travail (pp. 239-252). Paris : Syros « Collection Futuribles ».

- Bué, J. \& Roux-Rossi, D. (1993). Le travail de nuit des femmes dans l'industrie : les enseignements d'une étude monographique. Travail et Emploi, 56.

- Crittenden, A. (1994). Temporary solutions, Working Woman, 19, 32-35.

- Debouzy, M. (1979). Temps et sociétés : aspects du temps industriel aux Etats-Unis au début du XIX ${ }^{\mathrm{e}}$ siècle. Cahiers Internationaux de Sociologie, LXVII, 197-220.

- Découflé, A.-C. \& Svendsen, N. (1984). Contribution à une histoire des durées du travail dans l'industrie française du milieu du $\mathrm{XIX}^{\mathrm{e}}$ siècle, à la seconde guerre mondiale. Travail et Emploi, 20, 57-71.

- Doyelle, A. (1982). Durée du travail : Un essai de comparaison internationale. Travail et Emploi, 12, 9-23. 
- Engels, F. (1976). Von der Autorität. In G. Büschges (Eds.), Organisation und Herrschaft (pp. 55-58). Frankfurt : Rowohlt Taschenbuch Verlag (Edition originale, 1874). - Forté, M., Niss, M., Rebeuth, M.-C., Trautmann, J. \& Triby, E. (1998). De la division sexuée au partage du travail?, Travail et Emploi, 74, 51-62.

- Freyssinet, J. (1997). Le temps de travail en miettes, Paris : les Editions de l'atelier.

- Fridenson, P. (1993). Le temps de travail, enjeu de luttes sociales. In : J.-Y. Boulin, G. Cette, D. Taddei (Eds.), Le temps de travail (pp. 19-28). Paris : Syros «Collection Futuribles ».

- Friedberg, E. (1993). Le pouvoir et la règle. Paris : Seuil.

- Friedmann, G. (1963). Où va le travail humain ? Paris : Gallimard.

- Gasparini, G. (1990). Quelques observations sur les modèles culturels du temps dans les sociétés industrialisées contemporaines. Information sur les Sciences Sociales, 29, 725 743.

- Grossin, W. (1969). Le travail et le temps. Paris : Anthropos.

- Grossin, W. (1992). La création de l'Inspection du travail. Paris : L'Harmattan,.

- Grossin, W. (1994). Les temps de travail. In M. Coster \& F. Pichault (Eds.), Traité de sociologie du travail (pp. 127-144), Bruxelles : De Boeck Université.

- Grossin, W. (1996). Pour une science des temps : introduction à l'écologie temporelle.

Toulouse : Octarès.

- Groux, G. (1983). Les cadres. Paris : La Découverte/Maspero.

- Groux, G. \& Mouriaux, R. (1989). La CFDT. Paris : Économica.

- Groux, G. \& Mouriaux, R. (1992). La CGT. Crises et alternatives. Paris : Économica,.

- Guedj, F. \& Vindt, G. (1997). Le temps de travail : une histoire conflictuelle. Paris : Syros.

- Hartz, P. (1994). Jeder Arbeitsplatz hat ein Gesicht. Die Volkswagen-Lösung. Frankfurt/New York : Campus Verlag.

- Hartz, P. (1996). Das atmende Unternehmen. Jeder Arbeitsplatz hat einen Kunden Beschäftigungssicherung bei Volkswagen. Frankfurt/New York : Campus Verlag.

- Hébert, G. (1992). Traité de la négociation collective. Montréal : Presses Universitaires du Québec.

- Hirsch-Kreinsen, H. (1996). Allemagne, le retour des conseils des travailleurs ?. In : J. P. Durand (Ed.) Le syndicalisme au futur (pp. 95-116). Paris : Syros.

- La Chaise, G. (1996). Crise de l'emploi et fractures politiques. Paris : Presses de la Fondation Nationale des Sciences Politiques. 
- Lallement, M. (1993). L'organisation du temps de travail dans l'entreprise. In : J.-Y.

Boulin, G. Cette, D. Taddei (Eds.), Le temps de travail (pp. 193-204). Paris: Syros «Collection Futuribles ».

- Lallement, M. (1996). Sociologie des relations professionnelles. Paris : Éditions La Découverte.

- Lallement, M. \& Lefèvre, G. (1997). Le temps de travail des cadres, In : G. Bosch et al. (Eds.) Le temps de travail : nouveaux enjeux, nouvelles normes, nouvelles mesures (pp. 79109). Bruxelles : Editions du DULBEA.

- Le Goff, J. (1977). Pour un autre Moyen Age, Temps, travail et culture en occident. Paris : Gallimard.

- Marchand, O. \& Thelot, C. (1991). Deux siècles de travail en France. Paris : INSEE Etudes.

- Maruani, M. (1996). Le travail à temps partiel en Europe. In : H. Hirata \& D. Senotier (Eds.), Femmes et partage du travail (pp. 177-186). Paris : Éditions Syros.

- Marx, K. (1967). Le capital : critique de l'économie politique. Paris : Éditions Sociales (Edition originale 1867).

- Mercure, D. (1995). Les temporalités sociales. Paris : L'Harmattan.

- Morin, M.L., de Terssac, G. \& Thoemmes, J. (1998). La négociation du temps de travail : L'emploi en jeu. Sociologie du Travail, 2, 191-207.

- Naville, P. (1972). Temps et technique : structures de la vie de travail. Genève : Libraire Droz.

- Owen, J. (1989). Reduced working hours : cure for un employment or economic burden? Baltimore : The John Hopkins University Press.

- Pialoux, M. \& Beaud, S. (1993). Permanents et temporaires. In P. Bourdieu (Ed.), La misère du monde (pp. 317-329). Paris : Editons du Seuil.

- Promberger, M. (1994). Betriebliche Arbeitszeitpolitik in der Metallindustrie seit 1990, WSI-Mitteilungen, 3.

- Pronovost, G. (1996). Sociologie du temps. Bruxelles : de Boeck.

- Reynaud, J. D., Bernoux P. \& Lavorel L. (1966). Organisation syndicale, idéologie et politique de salaires. Sociologie du Travail, 66.

- Reynaud, J. D. (1989). Les règles du jeu : L'action collective et la régulation sociale. Paris : Armand Colin.

- Reynaud, J. D. (1996). Conflit et régulation sociale: Esquisse d'une théorie de la régulation conjointe, In J. D. Reynaud (Ed.), Le conflit, la négociation et la règle (pp. 4960). Toulouse : Octares (Edition originale 1979). 
- Rezsohazy, R. (1986). Les mutations sociales récentes et les changements de la conception du temps. Revue internationale de Sciences Sociales, 107, 37-52.

- Rosdücher, J. \& Seifert, H. (1994). Die Einführung der 4-Tage-Woche in der Volkswagen $A G$. Berlin : Senatsverwaltung für Arbeit und Frauen.

- Rosenberg, S. (1993). L'allongement du temps de travail aux Etats-Unis, In : J.-Y. Boulin, G. Cette, D. Taddei (Eds.), Le temps de travail (pp. 129-145). Paris: Syros «Collection Futuribles ».

- Savel, S. \& Gauthier, J.-P. (1998). La loi Aubry. Paris : Editions d'Organisation.

- Schor, J. (1991). The overworked american, New York : HarperCollins Publishers.

- Schwitzer, H. (1994). Der Weg zur 4-Tage-Woche. In : Peters J. (Ed.), Modellwechsel. Die IG Metall und die Viertagewoche bei VW, Göttingen : Steidl Verlag.

- Sue, R. (1994). Temps et ordre social. Paris : PUF.

- Supiot, A. (1995). Temps de travail : pour une concordance des temps, Droit social, 12, 947-954.

- Supiot, A.(1994). Critique du droit du travail. Paris : PUF.

- de Terssac, G., Morin, M.L., Thoemmes J. \& Sublet S. (1996). Les accords sur le temps de travail et la loi quinquennale du 20 décembre 1993 (rapport final). Toulouse : CERTOP-CNRS et LIRHE-CNRS.

- de Terssac, G. Thoemmes, J. Flautre, A. Thébault, C. (1998a). La mise en place du Compte Epargne Temps : modalités et enjeux ; note de synthèse. In : La négociation collective en 1997, Paris : Ministère de L'Emploi et de la Solidarité, La Documentation Française.

- de Terssac, G. Thoemmes, J. Flautre, A. Thébault, C. (1998b). La mise en oeuvre de la loi du 25 juillet 1994 : la construction des Comptes Epargne temps, Rapport final (Phase1), Paris : Ministère de l'emploi et de la solidarité.

- Thoemmes J. \& Labit A. (1995). La semaine des quatre jours chez Volkswagen : un scénario original de sortie de crise ? Travail et Emploi, 64, 5-23.

- Thoemmes, J. \& de Terssac G. (1997a). La construction des arrangements temporels : une analyse d'accord sur 11 ans dans une région, In : G. Bosch et al. (Eds.) Le temps de travail : nouveaux enjeux, nouvelles normes, nouvelles mesures (pp. 217-244). Bruxelles : Editions du DULBEA.

- Thoemmes, J. \& de Terssac G. (1997b). La négociation du temps de travail et les composantes du référentiel temporel, Loisir et Société, 20, 51-72.

- Thompson, E. P. (1967). Time, Work and Industrial Capitalism. Past and Present, 38, 56-97. 
- Tiano, A. (1988). Economie du travail. Paris : PUF.

- Tilly, C. (1991). Reasons for the continuing growth of part-time employment, Monthly

Labor Review, 3 .

- Tönnies, F. (1912). Gemeinschaft und Gesellschaft, Berlin : K. Curtis.

- Villermé, L.R. (1989). Tableau de l'état physique et moral des ouvriers employés dans les manufactures de coton, de laine et de soie. Paris : EDI. (Edition originale 1840).

- Weber, M. (1964). L'éthique protestante et l'esprit du capitalisme Paris : Plon. (Edition originale 1905).

- Weber, M. (1980). Wirtschaft und Gesellschaft : Grundrisse der verstehenden Soziologie. Tübingen : JBC Mohr. (Edition originale 1921).

- Weber, M. (1988). Methodologische Einleitung für die Erhebungen des Vereins für Sozialpolitik über Auslese und Anpassung (Berufswahl und Berufsschicksal) der Arbeiterschaft in der geschlossenen Grossindustrie (1908). In: M. Weber (Ed.). Gesammelte Aufsätze zur Soziologie und Sozialpolitik, (pp. 1-60). Tübingen : J.C.B. Mohr (Edition originale 1924).

- Weber, M. (1992). Essais sur la théorie de la science. Paris : Presses Pocket.

- Yates, M. (1994). Longer hours, fewer jobs. Employment and Unemployment in the United States, New York : Monthly Review Press,.

- Zarifian, P. (1996). La notion de «temps libre» et les rapports sociaux de sexe dans le débat sur la réduction du temps de travail, In : H. Hirata \& D. Senotier (Eds.), Femmes et partage du travail (pp. 25-39). Paris : Éditions Syros.

- Zarifian, P. (1996). Travail et communication. Paris : PUF. 